\title{
Messung und Modellierung der Initiatoreffektivität organischer Peroxide in der Ethen-Hochdruckpolymerisation
}

\author{
Dissertation \\ zur Erlangung des Doktorgrades \\ der Mathematisch-Naturwissenschaftlichen Fakultäten \\ der Georg August Universität zu Göttingen
}

vorgelegt von

Stefan Hinrichs

aus

Heide

Göttingen 2005 
D 7

Referent: Prof. Dr. M. Buback

Korreferent: Prof. Dr. K. Hoyermann

Tag der mündlichen Prüfung: 30.06.2005 


\section{INHALTSVERZEICHNIS}

1 ZUSAMMENFASSUNG.............................................................................................. 1

2 EINLEITUNG

Literaturverzeichnis von Kapitel 2............................................................................................... 5

\section{MATERIALIEN UND METHODEN ZUM STUDIUM DER INITIATOREFFEKTIVITÄT} IN DER ETHEN-HOMOPOLYMERISATION BEI HOHEN DRÜCKEN UND

Temperaturen und zUR Modellierung Mit PREDICI ................................. 6

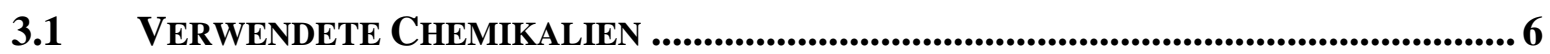

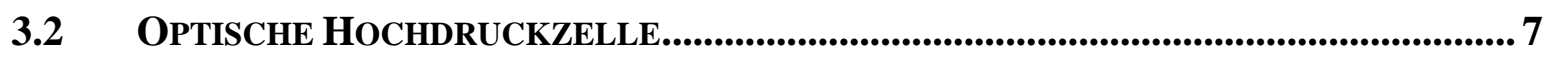

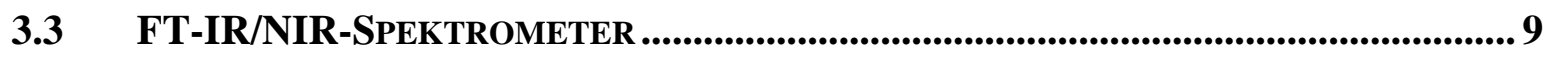

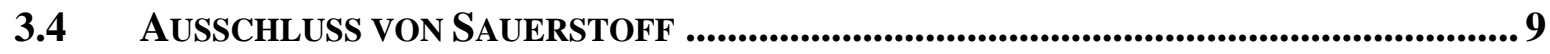

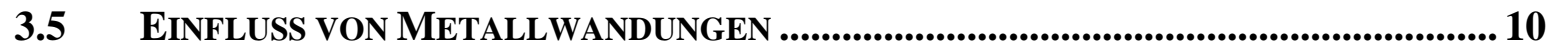

3.6 EXPERIMENTELLE ANORDNUNG ZUR KONTINUIERLICHEN HOCHDRUCK-

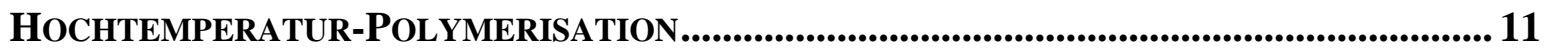

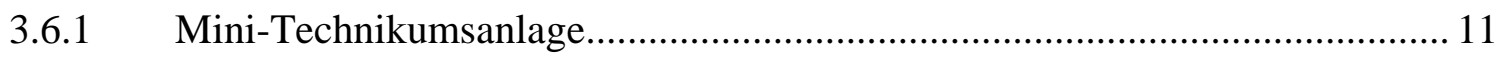

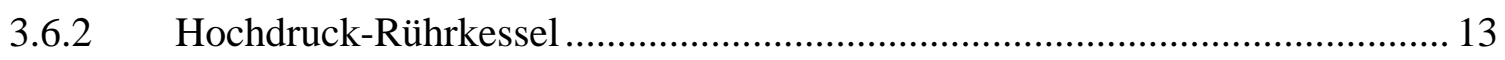

3.6.3 Dosierung des Molmassenreglers und der Initiatorlösung............................... 16

3.7 AUSWAHL DER REAKTIONSBEDINGUNGEN ................................................... 16

3.8 DURCHFÜHRUNG EINES TYPISCHEN EXPERIMENTS............................................... 18

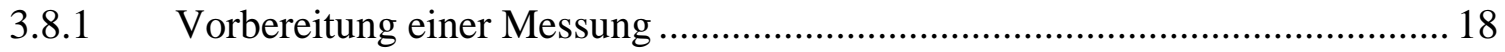

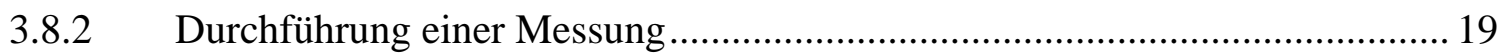

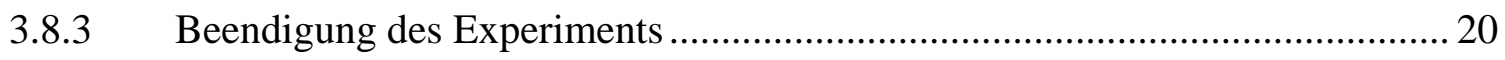

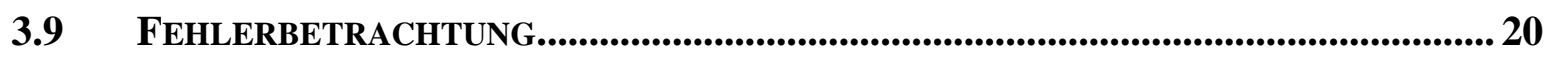

3.10 SIMULATIONSPROGRAMM Predici ............................................................. 21

3.11 LITERATURVERZEICHNIS FÜR KAPITEL 3................................................................. 22 


\section{Untersuchung DeR INITIATOREFFEKTIVITÄt ORgANischer PEROXIDE IN DER RADIKALISCHEN HOCHDRUCKPOLYMERISATION VON ETHEN}

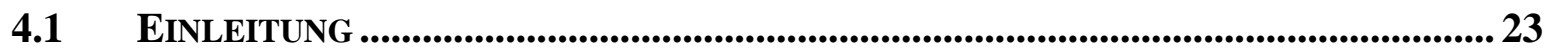

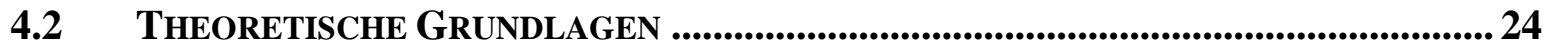

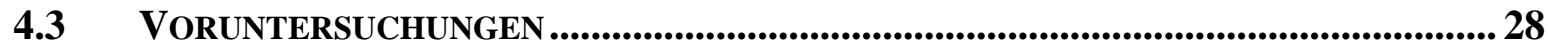

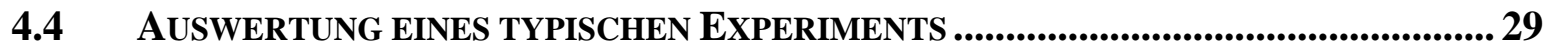

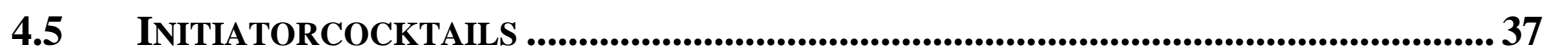

4.5.1 Initiierungsexperiment mit einer BTMHP/TBPO Mischung ........................... 38

4.5.2 Initiierungsexperiment mit einer TBPP/TBPA Mischung .............................. 40

4.6 ASYMMETRISCHE DIALKYLPEROXIDE ............................................................ 42

4.6.1 Initiierungsexperiment mit tert-Amyl-tert-butylperoxid als Initiator .............. 43

4.6.2 Initiierungsexperiment mit 1,1,2,2-Tetramethylpropyl-tert-butylperoxid als

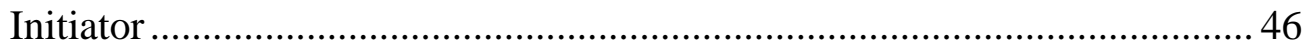

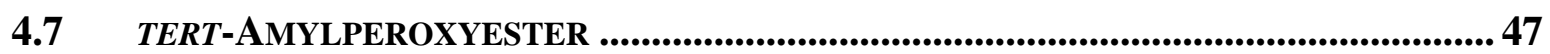

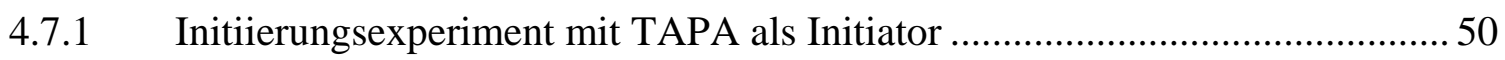

4.7.2 Initiierungsexperiment mit TAPnB als Initiator.......................................... 51

4.7.3 Initiierungsexperiment mit TAPiB als Initiator ........................................... 52

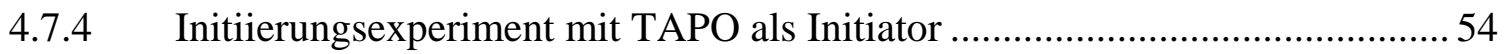

4.7.5 Initiierungsexperiment mit TAPP als Initiator ............................................... 55

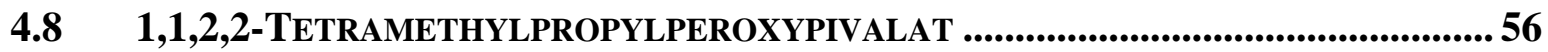

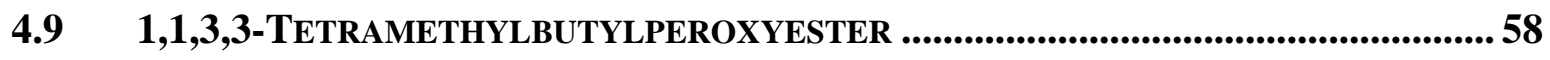

4.9.1 Initiierungsexperimente mit 1,1,3,3-Tetramethylbutylperoxyacetat................ 59

4.9.2 Initiierungsexperimente mit 1,1,3,3-Tetramethylbutylperoxy-2-ethylhexanoat 60

4.9.3 Initiierungsexperiment mit 1,1,3,3-Tetramethylbutylperoxypivalat ................ 61

4.10 DRUCKABHÄNGIGKEIT DER INITIATOREFFEKTIVITÄT ......................................... 63

4.10.1 Initiierungsexperimente mit Di-tert-butylperoxid..........................................64

4.10.2 Initiierungsexperimente mit tert-Butylperoxypivalat......................................65

4.10.3 Initiierungsexperimente mit tert-Butylperoxy-2-ethylhexanoat ......................67

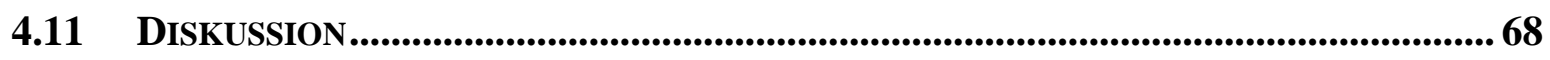

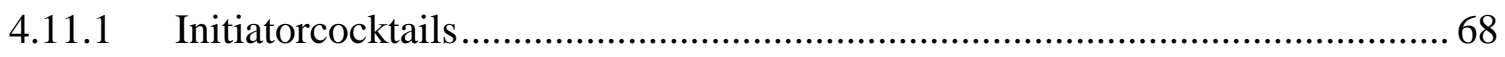

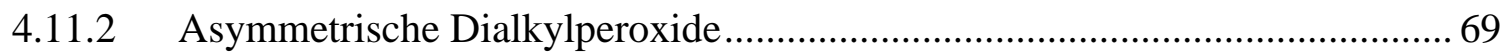




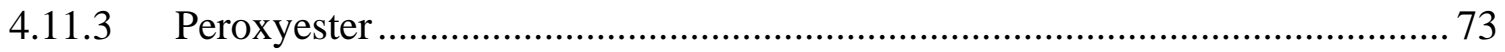

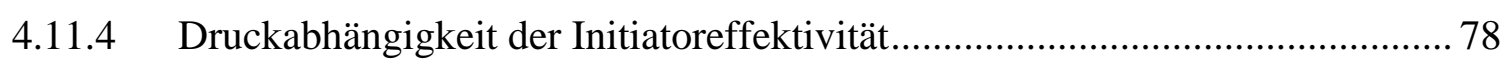

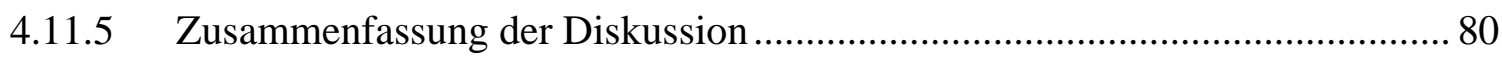

4.12 LITERATURVERZEICHNIS FÜR KAPITEL 4......................................................... 82

5 Modellierung VON KÄFigreaKTIONEN DURCH ANALYSE VON

ZERFALLSPRODUKTEN IN $N$-HEPTAN

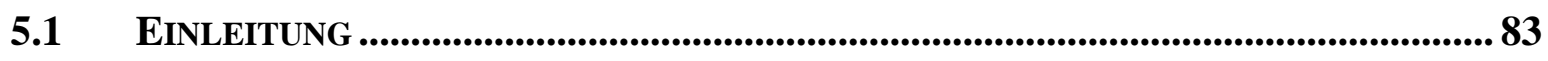

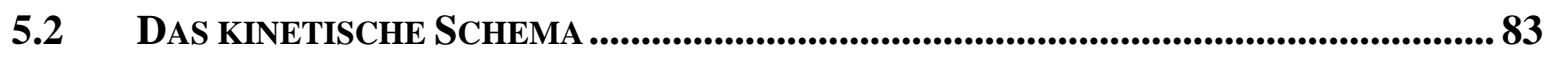

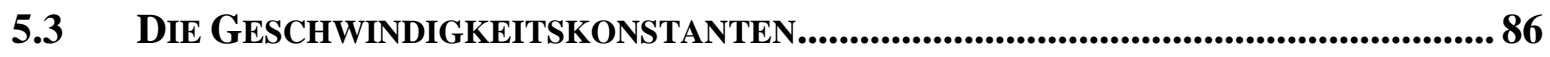

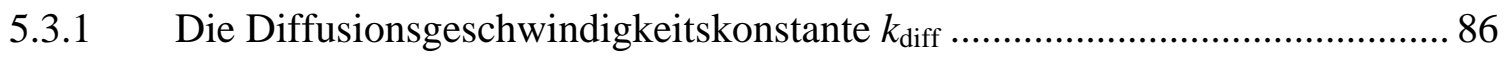

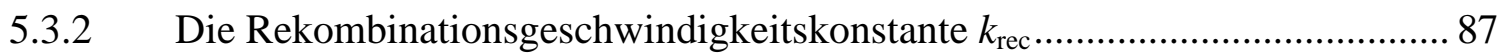

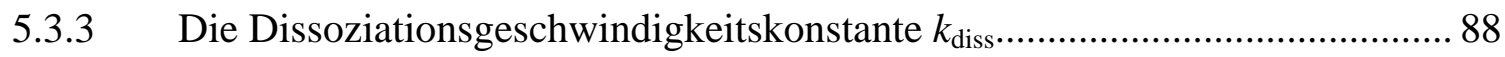

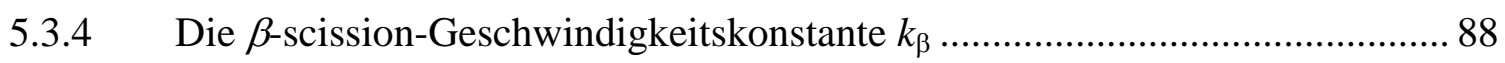

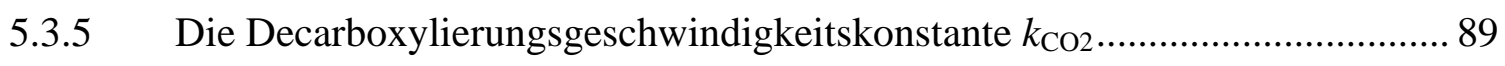

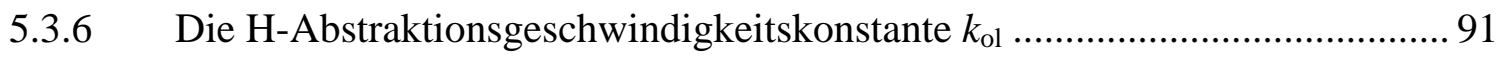

5.3.7 Die Kombinationsgeschwindigkeitskonstante $k_{\text {comb }}$ und die

Kreuzdisproportionierungsgeschwindigkeitskonstante $k_{\text {cross }}$....................... 91

\subsection{ERGEBNISSE DER MODELLIERUNG DER KÄFIGREAKTIONEN VON}

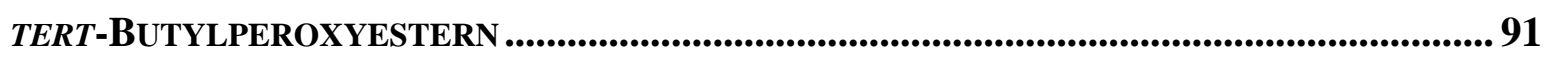

5.4.1 Modellierung der Temperatur- und Druckabhängigkeit der

Geschwindigkeitskonstanten für die H-Abstraktion von $n$-Heptan und die

Kombination des tert-Butoxy- und Methylradikals

5.4.2 Temperatur- und Druckabhängigkeit der Initiatoreffektivität abgeschätzt aus den Stoffmengenanteil derKäfigprodukten für tert-Butylperoxyacetat 95

5.4.3 Modellierung der Temperatur- und Druckabhängigkeit der

Geschwindigkeitskonstanten für die Kreuzdisproportionierung des tert-Butoxy- und $n$-Propylradikals 96

5.4.4 Temperatur- und Druckabhängigkeit der Initiatoreffektivität abgeschätzt aus dem Stoffmengenanteil der Käfigprodukte für tert-Butylperoxy-n-butyrat.. 98

5.4.5 Modellierung der Temperatur- und Druckabhängigkeit der

Geschwindigkeitskonstanten für die Kreuzdisproportionierung des tert-Butoxy- und iso-Propyl-radikals 
5.4.6 Temperatur- und Druckabhängigkeit der Initiatoreffektivität abgeschätzt aus dem Stoffmengenanteil der Käfigprodukte für tert-Butylperoxy-iso-butyrat

5.4.7 Modellierung der Temperatur- und Druckabhängigkeit der

Geschwindigkeitskonstanten für die Kreuzdisproportionierung des tert-Butoxy- und tert-Butylradikals 102

5.4.8 Temperatur- und Druckabhängigkeit der Initiatoreffektivität abgeschätzt aus dem Stoffmengenanteil der Käfigprodukte für tert-Butylperoxypivalat..... 103

5.5 DISKUSSION. 104

5.5.1 H-Abstraktiongeschwindigkeitskonstante des tert-Butoxyradikals von $n$-Heptan 105

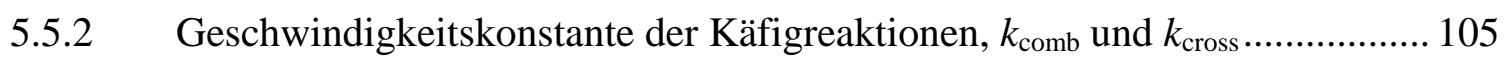

5.5.3 Die an Zerfallsprodukte modellierten Initiatoreffektivitäten der tert-Butylperoxyester 108

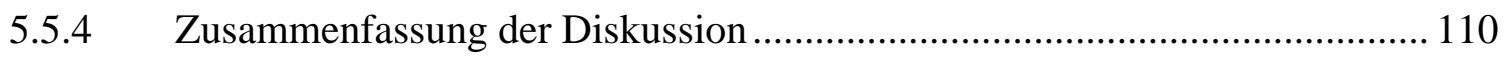

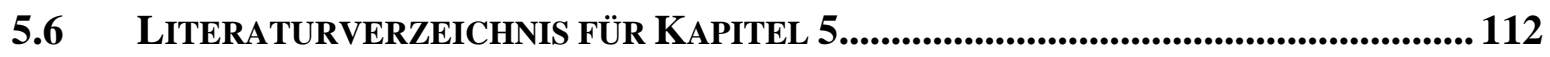

\section{MODELLIERUNG DER EXPERIMENTELL BESTIMMTEN}

INITIATOREFFEKTIVITÄTEN IN DER ETHEN-

HOCHDRUCKPOLYMERISATIONEN ........................................................... 113

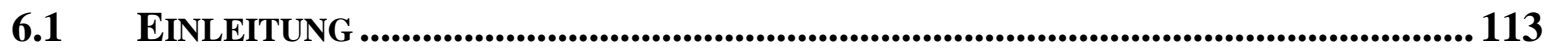

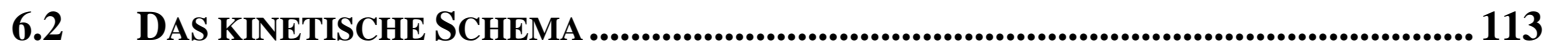

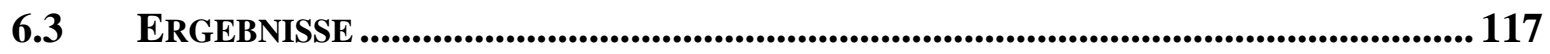

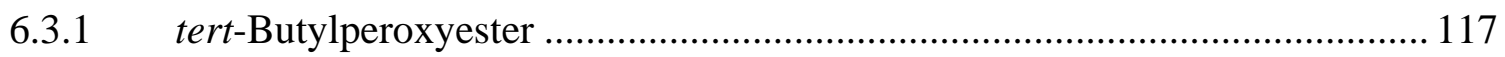

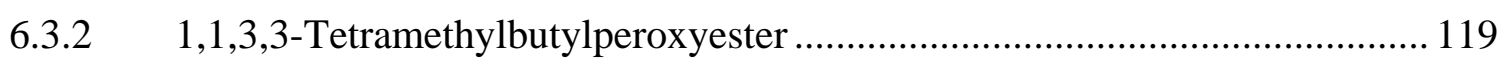

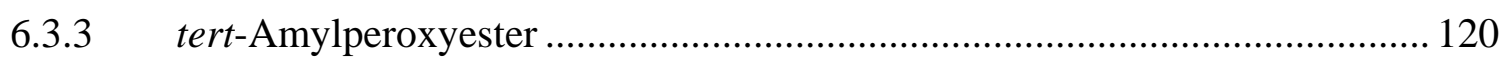

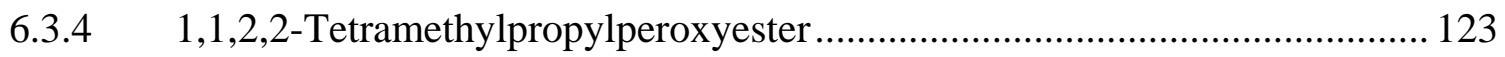

6.4 ZUSAMMENFASSUNG................................................................................................ 125

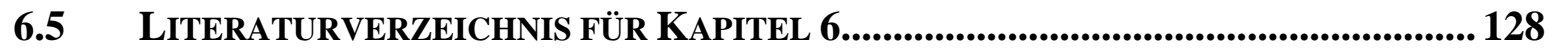

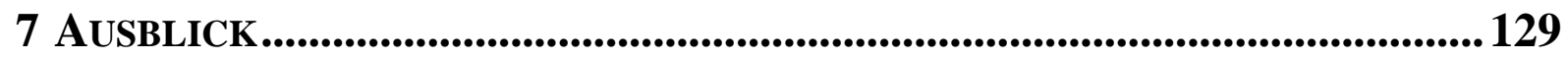


ANHANG

VERZEICHNIS DER ABKÜRZUNGEN .............................................................................. 130

UNTERSUCHUNG ZUR INITIATOREFFEKTIVITÄT........................................................ 134

BERECHNUNG DER KINETISCHEN KOEFFIZIENTEN UND DER DICHTE FÜR DAS MONOMERE

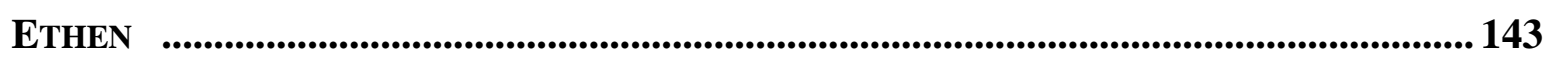




\section{Kapitel 1}

\section{Zusammenfassung}

Die Initiierungseffektivität in der Ethen-Hochdruckpolymerisation wurde in einem kontinuierlich betriebenen, nahezu idealen Rührkessel (CSTR) bei Temperaturen von 130 bis $250{ }^{\circ} \mathrm{C}$ und bei 2000 bar gemessen. Es wurden für asymmetrische Dialkylperoxide (tertButyl-O-O- $\left.\mathrm{R}_{1}\right)$ sowie verschiedene tert-Alkylperoxyester $\left(\mathrm{R}_{2}-(\mathrm{C}=\mathrm{O})-\mathrm{O}-\mathrm{O}-\mathrm{R}_{3}\right)$ Ethen-UmsatzTemperatur-Verläufe bestimmt. Der Ethen-Umsatz steigt mit der Polymerisationstemperatur zunächst an, durchläuft bei der optimalen Temperatur $T_{\text {opt }}$ ein Maximum und geht bei noch höheren Temperaturen zurück. Die Initiatoreffektivität $f$, das Verhältnis der Ketten startenden Radikale pro erzeugtes Primärradikal, wurde relativ zum Di-tert-Butylperoxid bestimmt.

Die Effektivität wird durch Variation der Substituenten $R_{1}, R_{2}$ und $R_{3}$ beeinflusst. Die Dialkylperoxide (Di-tert-Butylperoxid und Di-tert-Amylperoxid) besitzen eine Effektivität von eins, nur im Fall $\mathrm{R}_{1}=\mathrm{C}\left(\mathrm{CH}_{3}\right)_{2} \mathrm{C}\left(\mathrm{CH}_{3}\right)_{3}$ wurde $f=0.72$ erhalten.

Bei den tert-Alkylperoxyestern hat der Substituent $R_{2}$ den größten Einfluss auf die Effektivität. $f$ sinkt mit der Stabilität des $\mathrm{R}_{2}$-Radikals, welches durch Decarboxylierung entsteht. Nur bei den 1,1,2,2-Tetramethylpropylperoxyestern $\left[\mathrm{R}_{3}=\mathrm{C}\left(\mathrm{CH}_{3}\right)_{2} \mathrm{C}\left(\mathrm{CH}_{3}\right)_{3}\right]$ gibt es keinen Unterschied zwischen primärem und tertiärem Substituenten $\mathrm{R}_{2}(f=0.50)$. Beim Übergang von $\mathrm{R}_{3}=$ tert-Butyl zum 1,1,2,2-Tetramethylpropyl sinkt die Effektivität von 0.8 auf 0.5, wenn das zum Sauerstoffatom gebundene Kohlenstoffatom in $\mathrm{R}_{2}$ primär ist. Ist $\mathrm{R}_{2}$ hingegen tertiär, steigt $f$ bei dieser Variation von $\mathrm{R}_{3}$ von 0.4 auf 0.5 . Die gemessenen $f$-Werte können durch ein kinetisches Modell erklärt werden, welches auf der Annahme basiert, dass die Initiatoreffektivität durch das Ausmaß von Käfigreaktionen bestimmt wird. Zunächst wurde $f$ an experimentell erhaltene Stoffmengenanteile von Käfigprodukten der Peroxidzerfallreaktion in $n$-Heptan angepasst. Dies lieferte keine zufriedenstellenden Resultate, so dass die Geschwindigkeitskonstanten der Käfigreaktionen an die in der Ethen-Hochdruckpolymerisation bestimmten Effektivitäten angepasst wurden. Es wurde gezeigt, dass die Bildungsgeschwindigkeit der kohlenstoffzentrierten Radikale sowie die Reaktivität der zwei im Käfig befindlichen Radikale die Effektivität bestimmen. Besonders schnell ist die Reaktion von zwei primären kohlenstoffzentrierten Radikalen sowie die eines sauerstoffzentrierten Radikals mit einem tertiären kohlenstoffzentrierten Radikal.

Zusätzlich wurden Initiierungsexperimente im CSTR mit Initiatorcocktails durchgeführt. Zur Auswertung wurde eine Gleichung abgeleitet, mit der die Initiatoreffektivität für einen 
Initiator bestimmbar ist, wenn die des zweiten bekannt ist. Es konnte gezeigt werden, dass sich die Effektivitäten der untersuchten Initiatoren bei den verwendeten Konzentrationen nicht gegenseitig beeinflussen.

Es wurde erstmalig die Druckabhängigkeit der Initiatoreffektivität in der EthenHochdruckpolymerisation untersucht. Der Ethen-Umsatz steigt mit dem Druck. Die Initiatoreffektivität hingegen sinkt mit dem Druck und zwar umso stärker, je niedriger sie ist: für tert-Butylperoxypivalat von 0.60 auf 0.25 zwischen 1600 und 2500 bar, für tertButylperoxy-2-ethylhexanoat von 0.66 auf 0.34 und für Di-tert-butylperoxid von 0.97 auf 0.92 . 


\section{Kapitel 2}

\section{Einleitung}

Kunststoffe sind aufgrund ihrer vielfältig variierbaren Materialeigenschaften sehr verbreitete Werkstoffe, die zunehmend klassische Werkstoffe wie Gläser und Metalle verdrängen, da sie preiswerter sind bzw. bessere Eigenschaften aufweisen. Es werden beständig Herstellungsprozesse verbessert oder neu entwickelt, um hoch spezielle Polymere und Copolymere zu erhalten. Aber auch die bestehenden Produktionsprozesse für die Standardkunststoffe Polyethylen (PE), Polypropylen (PP), Polyvinylchlorid (PVC) oder Polystyrol (PS) werden hinsichtlich der Produktionskosten und Materialeigenschaften optimiert.

Das wichtigste technische Polymer ist Polyethylen mit einer Herstellungsmenge von 56 Millionen Tonnen im Jahr 2002 [1]. Man unterscheidet in Polyethylen hoher Dichte, das nach dem Verfahren von Ziegler und Natta durch Metallkatalyse hergestellt wird (HDPE = high density polyethylene), und Polyethylen niedriger Dichte, das unterteilt wird in LLDPE (linear low density polyethylene) und LDPE (low density polyethylene). LLDPE wird wie HDPE metallkatalytisch durch Copolymerisation von Ethen mit $\alpha$-Olefinen $\left(\mathrm{C}_{4}-\mathrm{C}_{8}\right)$ erhalten. LDPE hingegen wird durch radikalische Hochdruck-Polymerisation von Ethen hergestellt, die in Strömungsrohr- bzw. Rührkesselreaktoren bei Temperaturen von 150 bis $300{ }^{\circ} \mathrm{C}$ und Drücken von 1500 bis 3000 bar durchgeführt wird [2].

Um die radikalische Hochdruckpolymerisation $\mathrm{zu}$ optimieren, muss neben der Polymerisationskinetik des Monomers und der Kinetik des thermisch induzierten Zerfalls der eingesetzten Initiatoren die Initiatoreffektivität $f$ bekannt sein. Die Initiatoreffektivität gibt den auf die Anzahl entstehender Primärradikale bezogenen Anteil von Radikalen an, der eine wachsende Kette startet.

Als Initiatoren werden meist organische Peroxide verwendet, deren Aktivierungsenergie für die homolytische Spaltung der Peroxobindung zwischen 100 und $160 \mathrm{~kJ} \cdot \mathrm{mol}^{-1}$ liegt [3]. tert-Alkylperoxyester werden neben Diacyl- und Dialkylperoxiden besonders häufig als Initiatoren eingesetzt.

Trotz der großen Bedeutung von LDPE und der langen Erfahrung mit dem Herstellungsprozess (das Verfahren wurde in den dreißiger Jahren von ICI entwickelt [4]) gibt es nur wenige Daten zur Initiierungseffektivität organischer Peroxide in der Hochdruck- 
Polymerisation von Ethen. In den Studien von VAN DER MOLEN und VAN HeERDEN [5], sowie LuFT et. al. [6] wurden als Maß der Initiierungseffektivität die Größen Initiatorverbrauch und Initiatorproduktivität eingeführt. In den Arbeiten von BECKER [7] und SANDMANN [8] wurden Methoden vorgestellt, mit denen Initiatoreffektivitäten von organischen Peroxiden durch Monomer-Umsatzmessung direkt erhältlich sind.

Neben der Kenntnis der Initiatoreffektivität in der Ethen-Hochdruckpolymerisation ist es wichtig zu wissen, welche Prozesse $f$ beeinflussen. Aufbauend auf dieser Information besteht der Wunsch, organische Peroxide so auszuwählen, dass sie beispielweise eine hohe Effektivität bei tiefen Temperaturen aufweisen.

Einige der beim thermischen Zerfall entstehende Radikale können rasch miteinander reagieren, so dass dieser Prozess im Lösungsmittelkäfig erfolgt. Die entstandenen Verbindungen werden als Käfigprodukte bezeichnet. Durch die Reaktion wird die Anzahl an Radikalen herabgesenkt, so dass die Initiatoreffektivität in der Polymerisation ebenfalls kleiner sein sollte. Es wurde für tert-Butylperoxyacetat (TBPA) gezeigt [9], dass der im Zerfallsexperiment bei $190{ }^{\circ} \mathrm{C}$ und 2000 bar in $n$-Heptan erhaltende Stoffmengenanteil $x=$ 0.20 des Käfigprodukts tert-Butylmethylether mit der Initiatoreffektivität $f_{\text {tthen }}=0.79$ zusammenhängt.

Da die Effektivität durch Verlustreaktionen der Radikale im Lösungsmittelkäfig bestimmt wird, soll im Rahmen dieser Arbeit ein kinetisches Modell der Käfigreaktionen aufgestellt werden, mit dem der Stoffmengenanteil der Käfigprodukte und die Initiatoreffektivität berechnet werden kann. Dieses Modell erlaubt es, Aussagen zu machen, welche Reaktionen verantwortlich für die Absenkung der Initiatoreffektivität sind.

Außerdem soll im Rahmen dieser Arbeit mit einem kontinuierlich betriebenen Rührkesselreaktor (CSTR $=$ continous stirred tank reactor) die Initiatoreffektivität $f$ von organischen Peroxiden bestimmt werden. 


\section{Literaturverzeichnis für Kapitel 2}

[1] U. Beginn, H. Keul, D. Klee, M. Möller, A. Mourran, K. Peter, O. Weichold, T. Dreier, Nachr. Chem. 52 (2004) 324

[2] Ullmann's encyclopedia of industrial chemistry A 23, Reprint, VCH, Weinheim (1995)

[3] W. Ando, Ed., Organic Peroxides, John Wiley and Sons, New York (1992)

[4] R. Mühlhaupt, Nachr. Chem. Tech. Lab. 41 (1993) 1344

[5] T. J. van der Molen, C. van Heerden, Advances in Chemistry Series, Ed. K. B. Bischoff, American Chemical Society, Washington (1972) 92

[6] G. Luft, H. Bitsch, H. Seidl, J. Macromol. Sci. Chem. A11 (1977) 1089

[7] P.Becker, Dissertation, Göttingen (2000)

[8] J. Sandmann, Dissertation, Göttingen (2000)

[9] D. Nelke, Diplomarbeit, Göttingen (1998) 


\section{Kapitel 3}

\section{Materialien und Methoden zum Studium der}

\section{Initiatoreffektivität in der Ethen-Homopolymerisation bei}

hohen Drücken und Temperaturen und zur Modellierung mit Predici

\subsection{Verwendete Chemikalien}

In Tabelle 3.1 sind die im Rahmen dieser Arbeit verwendeten Chemikalien mit dem vom Hersteller angegebenen Reinheitsgrad aufgelistet. Wenn keine weiteren Angaben gemacht werden, wurden die Substanzen ohne weitere Aufarbeitungsschritte eingesetzt. Der Reinheitsgrad der organischen Peroxide wurde vom Hersteller durch die iodometrische Bestimmung des Gehalts an aktivem Sauerstoff (Peroxo-Funktion) bestimmt. Die Aufbewahrung der Initiatoren erfolgte bei $-20^{\circ} \mathrm{C}$, da die Peroxide bei dieser Temperatur so langsam zerfallen, dass sie in der Regel ohne nachweisbare Zersetzung für mehrere Monate gelagert werden können.

Der Kettenüberträger Propionaldehyd wurde mittels eines Degassers (ERC $3215 \alpha$ Degasser, ERC, Altegolfsheim (Regensburg)) entgast. Die Lösung der Initiatoren in $n$-Heptan wurde durch Umgasen mit Stickstoff von Sauerstoff befreit. 


\begin{tabular}{|c|c|c|c|}
\hline Chemikalie & Abkürzung & Reinheitsgrad / \% & Hersteller \\
\hline tert-Amyl-tert-butylperoxid & TATBP & 99 & AKZO NOBEL \\
\hline $\begin{array}{c}\text { 1,1,2,2-Tetramethylpropyl- } \\
\text { tert-butylperoxid }\end{array}$ & ТМРТВР & 96.8 & AKZO NOBEL \\
\hline Di-tert-butylperoxid & DTBP & 99 & AKZO NOBEL \\
\hline Bistrimethylhexanoylperoxid & ВТМHP & 75 & AKZO NOBEL \\
\hline tert-Butylperoxy-2-ethylhexanoat & TBPO & 98.6 & AKZO NOBEL \\
\hline tert-Butylperoxyacetat & TBPA & 99.4 & AKZO NOBEL \\
\hline tert-Butylperoxypivalat & ТВРP & 99 & AKZO NOBEL \\
\hline tert-Amylperoxyacetat & TAPA & 98.9 & AKZO NOBEL \\
\hline tert-Amylperoxy- $n$-butyrat & TAPnB & 98 & AKZO NOBEL \\
\hline tert-Amylperoxy-iso-butyrat & TAPiB & 98 & AKZO NOBEL \\
\hline tert-Amylperoxy-2-ethylhexanoat & TAPO & 98 & AKZO NOBEL \\
\hline tert-Amylperoxypivalat & TAPP & 75 & AKZO NOBEL \\
\hline $\begin{array}{c}\text { 1,1,2,2-Tetramethylpropyl- } \\
\text { peroxypivalat }\end{array}$ & TMPPP & 90.9 & AKZO NOBEL \\
\hline 1,1,3,3-Tetramethylbutylperoxyacetat & TMBPA & 50 & AKZO NOBEL \\
\hline $\begin{array}{l}\text { 1,1,3,3-Tetramethylbutyl- } \\
\text { peroxy-2-ethylhexanoat }\end{array}$ & TMBPO & 90.9 & AKZO NOBEL \\
\hline 1,1,3,3-Tetramethylbutylperoxypivalat & TMBPP & 75 & AKZO NOBEL \\
\hline Ethen & $\mathrm{E}$ & 99.8 & Linde \\
\hline Propionaldehyd & PA & 98 & Fluka AG \\
\hline$n$-Heptan & & $\geq 99$ & Haltermann \\
\hline
\end{tabular}

Tab. 3.1: Verwendete Chemikalien mit dem vom Hersteller angegebenen Reinheitsgrad.

\subsection{Optische Hochdruckzelle}

Die für die kontinuierliche Reaktionstechnik verwendete optische Hochdruckzelle (Abbildung 3.1) ist bis zu Drücken von 3500 bar und Temperaturen von $300{ }^{\circ} \mathrm{C}$ einsetzbar. 


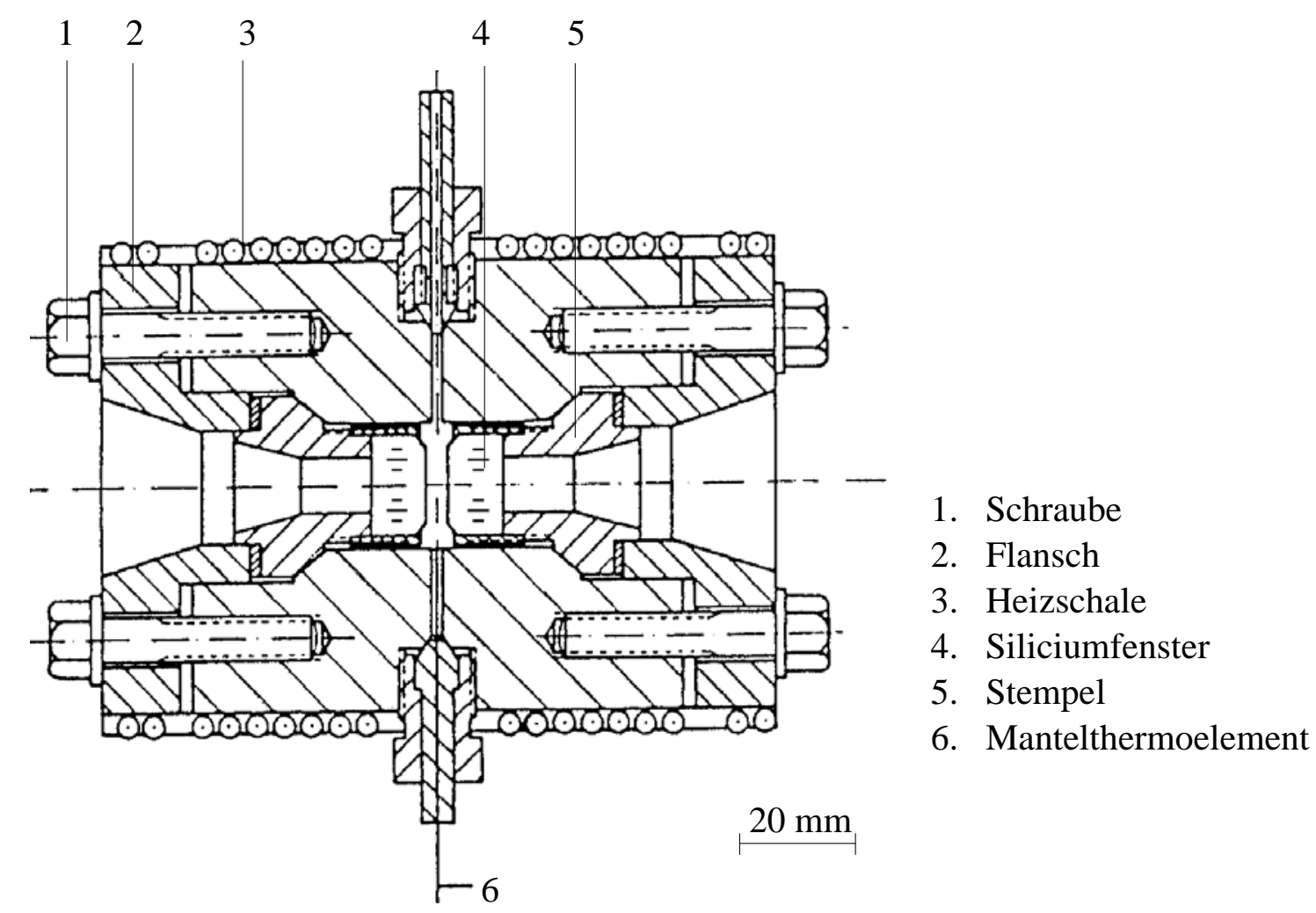

Abb. 3.1: Optische Hochdruckzelle

Der hohlzylindrische Zellkörper besteht aus einer hochwarmfesten Nickelbasislegierung (Werkstoff RGT 601, Werkstoff-Nr. 2.4668, Arbed Saarstahl), hat eine Länge von 100 mm, einen Außendurchmesser von 80 mm und einen Innendurchmesser von 22 mm. Die Dichtung erfolgt durch konische Stempel (5) (Werkstoff RGT 12, Werkstoff-Nr. 2.4969, Arbed Saarstahl), die durch Flansche (2) gegen die Zellkonen gepresst werden. Durch Auswahl von unterschiedlich langen Stempeln kann die optische Schichtlänge zwischen den drucktragenden Siliciumfenstern von weniger als einem Millimeter auf mehrere Millimeter variiert werden.

Zwei seitliche Bohrungen zum Anschluss von Hochdruckkapillaren ermöglichen das Befüllen und Entleeren der Zelle bzw. ihre Verwendung als kontinuierlich durchströmte Analysenzelle. Zur Temperaturmessung wird ein Thermoelement (6) (Nickel-Chrom gegen Nickel-ChromelAlumel, CIA S250, CGE Alsthom) über eine von zwei weiteren Bohrungen direkt bis an die optische Messschicht eingeführt. Nicht benötigte Kapillarbohrungen werden durch Blindstopfen verschlossen.

Die Heizung der Hochdruckzelle erfolgt elektrisch mit Mantelheizleitern (3) (Pyrolyn-M, $16 \Omega \mathrm{m}^{-1}$, Les Cables de Lyon), die in einer über die Zelle geschobenen Messingmatrix eingelassen sind. Die Temperatur wird während eines Experiments über einen PID-Regler (Eurotherm 820) auf $\pm 0.3 \mathrm{~K}$ konstant gehalten. 
Die optischen Hochdruckfenster (4) bestehen aus polykristallinem Reinstsilicium (Korth), das im Spektralbereich von $1450-7000 \mathrm{~cm}^{-1}$ eine ausreichend gute Durchlässigkeit besitzt. Zwischen Siliciumfenster und polierter Auflagefläche der Stempel befindet sich zum Ausgleich von Oberflächenunebenheiten eine $12 \mu \mathrm{m}$ dünne Folie aus Teflon, auf der das Hochdruckfenster durch eine Überwurfkappe fixiert wird.

\subsection{FT-IR/NIR-Spektrometer}

Zur Aufnahme von Infrarot- und Nahinfrarotspektren stand ein FouriertransformSpektrometer (Bruker IFS 88) zur Verfügung. Der Probenraum ist zur besseren Aufnahme der Hochdruckzellen gegenüber der Standardausführung etwas verändert worden. Er besitzt eine wärmeableitende, kühlwasserdurchspülte Zellhalterung sowie eine vergrößerte Abdeckung und wird kontinuierlich mit wasser- und kohlendioxidarmer Luft gespült.

Die optische Konfiguration für die hier beschriebenen Messungen besteht aus einer Globar-Strahlungsquelle und einem mit Silicium beschichteten Calciumfluorid-Strahlteiler. Für die Messungen wurde ein mit flüssigem Stickstoff gekühlter MCT-Detektor eingesetzt, der eine hohe Lichtempfindlichkeit besitzt und die schnelle Aufnahme der Spektren ermöglicht. Die Spektrenaufnahme und -verarbeitung erfolget über einen IBM-kompatiblen Personalcomputer. Als Software wurde das Programm Opus 3.0 der Firma Bruker verwendet. Während eines Experiments wurden Spektren im Wellenzahlbereich von 1500 bis $8000 \mathrm{~cm}^{-1}$ aufgenommen. Dazu wurden 100 Interferogramme coaddiert und abgespeichert. Zur Apodisierung vor der Fourier-Transformation wurde eine Blackman-Harris 3-Term Funktion verwendet. Aus dem so erhaltenen Ein-Kanal-Spektrum wurde mit Hilfe eines vorher aufgenommenen Ein-Kanal-Referenzspektrums das Absorbanz-Spektrum berechnet und abgespeichert.

\subsection{Ausschluss von Sauerstoff}

Das Sauerstoffmolekül kann als Diradikal wesentlich in radikalische Reaktionsmechanismen beim Initiatorzerfall eingreifen. Aus verschiedenen, in der Literatur beschriebenen Experimenten ist bekannt, dass dabei sowohl die Kinetik als auch die Selektivität des Zerfalls von organischen Peroxidverbindungen beeinflusst werden kann [1,2]. So kann ein Sauerstoffradikal z. B. mit dem aus einem Peroxyester gebildeten Alkylradikal kombinieren. 
Dieses Peroxyradikal kann sich anschließend durch Abstraktion eines Wasserstoffatoms aus dem Lösungsmittel zum Hydroperoxid stabilisieren:

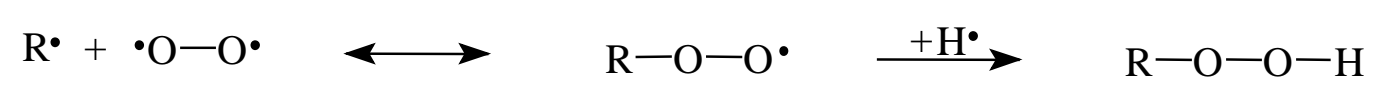

Abb. 3.2: Reaktion von Alkylradikal und Sauerstoff zum Hydroperoxid.

Das entstehende Hydroperoxid ist im Regelfall wesentlich stabiler als das ursprünglich vorhandene Peroxid, zerfällt nach einem anderen Mechanismus und kann sowohl zu einem zusätzlichen induzierten Zerfall als auch zur Bildung anderer Produkte führen

Es wurde daher versucht, möglichst unter Ausschluss von Luft und somit auch unter Ausschluss von Sauerstoff zu arbeiten. Der Kettenüberträger Propionaldehyd wurde mittels eines Degassers (ERC 3215 a Degasser, ERC, Altegolfsheim (Regensburg)) entgast. Die Lösung der Initiatoren in $n$-Heptan wurde durch Umgasen mit Stickstoff von Sauerstoff befreit.

\subsection{Einfluss von Metallwandungen}

Es ist bekannt, dass viele Metalle und ihre Salze, besonders Nebengruppenelemente und die aus ihnen gebildeten organometallischen Komplexe, die Zersetzung von Peroxiden beschleunigen [3, 4].

Die zum Aufbau der Hochdruckapparaturen verwendeten Materialien bestehen aus metallischen Legierungen, die größere Mengen an Eisen, Kobalt und Nickel enthalten. Bei den Messungen kommt die Peroxidlösung in direkten Kontakt mit diesen Materialien, so dass eine Beeinflussung der Ergebnisse durch Wandreaktionen zu erwarten ist. Durch den katalytischen Effekt des Reaktorwandmaterials ist eine Beschleunigung der Zerfallsreaktion, vor allem bei niedrigen Temperaturen, zu erwarten, da ein Katalysator die Aktivierungsenergie einer chemischen Reaktion herabsetzt. Dieser Effekt sollte mit steigender Temperatur gegenüber der unkatalysierten Reaktion zunehmend an Gewicht verlieren. In früheren Arbeiten [5, 6] wurde gezeigt, dass es möglich ist, die Temperaturabhängigkeit der kinetischen Koeffizienten sowohl aus "metallfreien" Innenzellenexperimenten, als auch aus kontinuierlichen Messungen durch eine gemeinsame Arrhenius-Gerade zu beschreiben. Daraus wurde gefolgert, dass bei den hohen Temperaturen, die im CSTR herrschen, und den damit verbundenen kurzen 
Reaktionshalbwertszeiten eine katalytische Wandreaktion keinen nennenswerten Einfluss auf die eigentliche Zerfallsreaktion besitzt.

\subsection{Experimentelle Anordnung zur kontinuierlichen Hochdruck- Hochtemperatur-Polymerisation}

\subsubsection{Mini-Technikumsanlage}

Im Rahmen der vorliegenden Arbeit wurde die im Arbeitskreis vorhandene MiniTechnikumsanlage, die BECKER [7] und SANDMANN [8] in Anlehnung einer bereits bestehenden Anlage aufgebaut haben [9, 10, 11], für Polymerisationsexperimente eingesetzt. In diesem Abschnitt wird eine Übersicht des Aufbaus des Mini-Technikums gegeben.

Im Gegensatz zu früheren Arbeiten, in denen weitestgehend Sauerstoff freies Ethen zur Verfügung stand, wird im Rahmen der vorliegenden Arbeit Ethen vor Ort mittels eines Katalysators (BASF, R 3-11, Kupfer in hochdisperser Form, Bindung des Sauerstoffs in oxidischer Form) gereinigt und anschließend über einem Molsieb von eventuell vorhandenen Spuren Wasser befreit. Somit kann unabhängig von der Güte einzelner Ethen-Chargen eine gleichbleibend gute Qualität des verwendeten Ethens gewährleistet werden. In Abbildung 3.3 ist der schematische Aufbau der Apparatur gezeigt. 


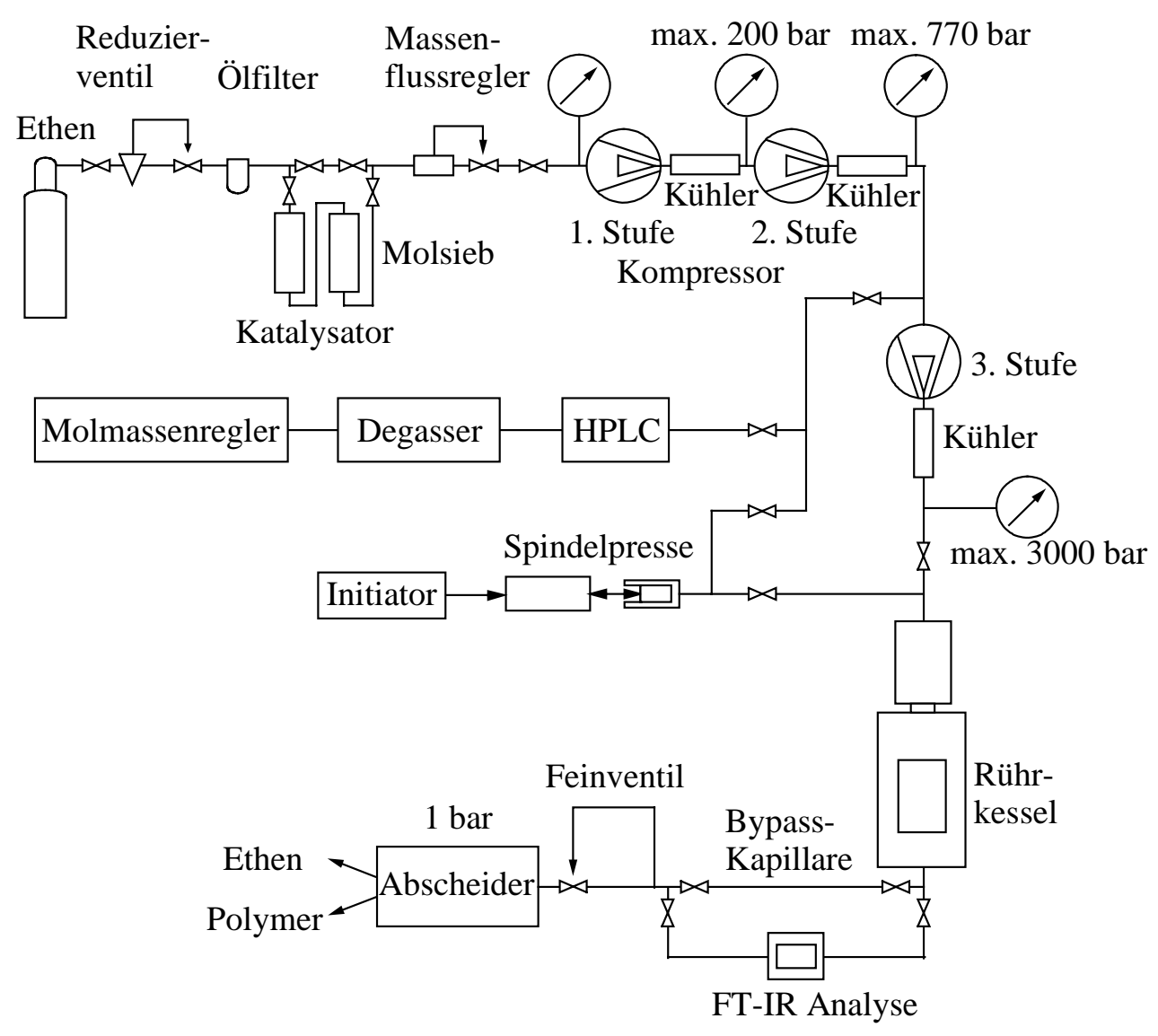

Abb. 3.3: Schematische Darstellung der Mini-Technikumsanlage.

Das Ethen strömt, nachdem es mit einem auf $40^{\circ} \mathrm{C}$ geheizten Rohrdruckminderer vom Flaschendruck auf ca. 10 bar entspannt worden ist (für diese Bedingungen wurde der Massenflussregler vom Hersteller kalibriert), durch einen Ölfilter (Porenweite, $0.01 \mu \mathrm{m}$, Riegler, Bad Urach). In der Katalysatorstufe wird das Ethen von Sauerstoff befreit und getrocknet. Ein Massenflussregler (5851 E, Brooks, Mannheim) bestimmt und regelt den Ethenmassenfluss entsprechend einer externen Sollwertvorgabe. Die Regelgenauigkeit beträgt $1 \%$ bei einer Reproduzierbarkeit des Skalenendwertes von 0.2 \%. Der Einsatzbereich des Massenflussreglers erstreckt sich bis $2 \mathrm{~kg} \cdot \mathrm{h}^{-1}$.

Im normalen Betrieb wird das Ethen von einem zweistufigen Vorverdichter (Hofer, Mühlheim/Ruhr) von 10 auf ca. 250 bar und einem einstufigen Nachverdichter (Hofer, Mühlheim/Ruhr) auf einen Druck von maximal 3000 bar komprimiert. Vor und hinter der Nachverdichterstufe können mit Hilfe verschiedener Dosiersysteme flüssige Additive, wie Molmassenregler und Initiatorlösung, in das Strömungssystem eingebracht werden. Eine detaillierte Beschreibung der Dosiersysteme findet sich bei MäHLING [12]. Die so hergestellte Reaktionsmischung gelangt über Kapillaren in den geheizten Rührkessel, in dem die Umsetzung erfolgt. Die Temperatur der Hochdruckkomponenten, die dem Rührkessel 
nachgeschaltet sind, liegt bei ca. $140^{\circ} \mathrm{C}$, um eine Phasentrennung der Reaktionsmasse zu vermeiden. Die Weiterreaktion in diesem Teil der Anlage kann aufgrund der sehr kurzen Verweilzeit vernachlässigt werden.

Die Reaktionsmischung strömt, je nach Wunsch, durch eine Bypass-Kapillare oder durch eine optische Hochdruckzelle, die in einem FT-IR-Spektrometer justiert ist (siehe Abschnitt 3.3). Sie entspricht der in Abschnitt 3.2 vorgestellten Zelle. In dieser Zelle kann der Umsatz und die Gemischzusammensetzung on-line IR/NIR-spektroskopisch analysiert werden. So kann das Vorliegen eines stationären Betriebszustands kontrolliert werden. Nach Passieren der optischen Hochdruckzelle wird das Reaktionsgemisch in einem Schritt von 2000 bar auf 1 bar entspannt. Hierzu wird ein, in der mechanischen Werkstatt des Instituts für Physikalische Chemie der Universität Göttingen angefertigtes, Feinventil verwendet [10]. Die über das Feinventil entspannte Reaktionsmischung gelangt über eine kurze Hochdruckkapillare in eine Normaldruck-Abscheidevorrichtung [13]. In dieser Vorrichtung wird das Polymerisat von gasförmigen Produkten getrennt und quantitativ aufgefangen.

Zur Temperaturmessung im Reaktor, in der optischen Hochdruckzelle, an den Kapillaren und dem Feinventil werden Thermoelemente (Nickel-Chrom gegen Nickel-Chrom-Alumel, CIA S250, CGE Alsthom) eingesetzt. Die Temperaturmessung im Reaktor erfolgt an drei Stellen (am Reaktoreinlass, unter dem Rührer und direkt vor dem Reaktorauslass), so dass eventuell auftretende Temperaturgradienten erkannt werden können. Zusätzlich wird die Temperatur im Reaktormantel ( $T_{\text {Mantel }}$ ) gemessen. Über diese Messstelle wird der PID-Regler für die Regelung der Reaktortemperatur angesprochen. Die Heizung des Reaktors, der optischen Hochdruckzelle und des Feinventils erfolgt elektrisch mit Mantelheizleitern (Pyrolon-M, $16 \Omega$ $\mathrm{m}^{-1}$, Les Cables de Lyon), die in einer über die Bauteile geschobenen Messingmatrix eingelassen sind. Für die Regelkreise der Betriebstemperaturen werden PID-Regler (Eurotherm, Typ 2208) verwendet. Die Druckmessung erfolgt mit DMS-Druck-Umwandlern (HBM-Messtechnik, Klasse 2, Maximaldruck 3 kbar) vor dem Rührkessel. Eine ausführlichere Beschreibung des Aufbaus findet sich bei BuscH [11].

\subsubsection{Hochdruck-Rührkessel}

Der Hochdruck-Rührkessel (siehe Abbildung 3.4) ist das Kernstück der beschriebenen Hochdruckapparatur. Im Rahmen dieser Arbeit wurde ausschließlich der von LoviS entwickelte und charakterisierte Käfig-Propeller-Rührer eingesetzt. Mit diesem Rührer ist der Rührkessel als annähernd idealer Rührkessel anzusehen [14]. Der Autoklav besitzt unter Berücksichtigung aller Einbauten ein Innenvolumen von $54.23 \mathrm{~cm}^{3}$. Er besteht aus einem 
zylindrischen Hohlkörper von 170 mm Länge und hat einen Außendurchmesser von 150 mm. Der Innendurchmesser beträgt $42 \mathrm{~mm}$. Der Autoklavenkörper, sowie unterer und oberer Flansch, wurden aus einer hochwarmfesten Nickelbasislegierung (RGT 601, Werkstoff-Nr. 2.4668, Arbed-Saarstahl) gefertigt. Das Verhältnis von Innen- zu Außendurchmesser in Verbindung mit dem gewählten Material gestattet unter Berücksichtigung eines Sicherheitsbeiwertes von 2.5 eine maximale Druckbelastung von 3000 bar bei $300{ }^{\circ} \mathrm{C}$. Abgedichtet wird die Unterseite des Reaktors durch einen Stempel (RTG 12, Werkstoff-Nr. 2.4969, Arbed-Saarstahl) mit einer Metall-Metall-Konendichtung. Der Stempel wird durch den unteren Flansch mit zwölf Dehnbolzen bei einem Drehmoment von jeweils 90 Nm auf den Autoklaven-Hauptkörper gepresst. Die Verwendung von Dehnbolzen verhindert bei hohen Drucksprüngen, die zu plastischen Verformungen des Metalls führen können, eine Beschädigung der Gewindeschäfte im Reaktorblock. An der oberen Seite wird der Reaktor durch eine Dichtlinse (17) (RTG 12) ebenfalls über Konendichtungen abgeschlossen. Die Linse ist axial durchbohrt, um den Rührer über eine Achse mit der Rührwelle zu verbinden. Der Innendurchmesser zur Rührantriebsseite beträgt $11 \mathrm{~mm}$, zur Reaktorseite $22 \mathrm{~mm}$. In diesem Teil befinden sich ein gekapseltes Edelstahlkugellager und eine Befestigungsvorrichtung für den Rührer. Das Lager wird mit einer Überwurfkappe in der Dichtlinse festgehalten. Der Antrieb des Rührers erfolgt über eine HochdruckMagnetkupplung (Autoclave Engineers). 


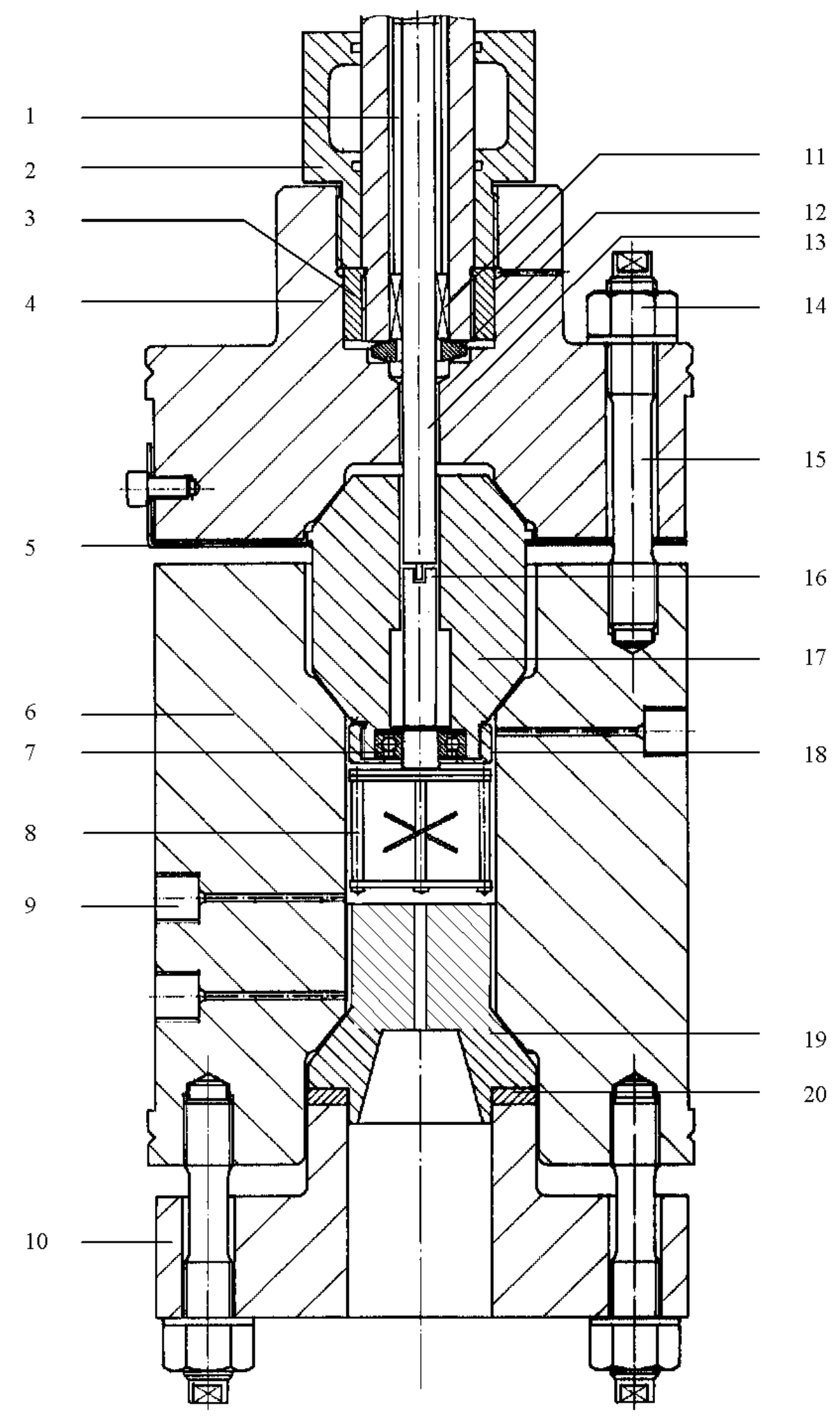

Abb. 3.4: Hochdruck-Rührkessel: 1 Magnetantrieb, 2 Andruckmutter, 3 Kontermutter, 4 oberer Flansch, 5 Halteblech für Dichtlinse, 6 Autoklavenkörper, 7 Rührlager, 8 Rührer, 9 Bohrung (z.B. für Thermoelement), 10 unterer Flansch, 11 Lager für Antriebswelle, 12 Dichtlinse, 13 Antriebswelle, 14 Mutter, 15 Dehnbolzen, 16 Kopplung für Antriebswelle an Rührer, 17 Dichtlinse, 18 Überwurfkappe, 19 Stempel, 20 Gleitring 
Um den Wärmefluss vom geheizten Reaktor zur Magnetkupplung zu verhindern, ist der Verbindungsflansch wassergekühlt. Der Antrieb der äußeren Permanentmagneten erfolgt über eine Riemenscheibe und einen Synchro-Servo-Motor (Seidel) mit entsprechender Regelelektronik. Es lassen sich Drehzahlen bis $1000 \mathrm{U} \cdot \mathrm{min}^{-1}$ stufenlos einstellen und über eine Hall-Sonde kontrollieren.

Detailliertere Informationen über die Konstruktion des Autoklaven, des Rührers und des Rührantriebes finden sich bei BUSCH [11], BUBACK et al. [10] und LOVIS [14].

\subsubsection{Dosierung des Molmassenreglers und der Initiatorlösung}

Die Dosierung des Molmassenreglers Propionaldehyd erfolgte mit einer elektrischen HPLCPumpe (Maxi-Star K-1001, Knauer) direkt vor der Nachverdichterstufe, nachdem der Propionaldehyd mit einem Degasser (ERC 3215 a Degasser, ERC, Altegolfsheim (Regensburg)) entgast worden ist. Die genaue Förderleistung der HPLC-Pumpe wurde während des gesamten Experiments gravimetrisch mittels einer Analysenwaage (Sartorius) kontrolliert.

Die Dosierung der Initiatorlösung erfolgte bei einem konstanten Druck von 2000 bar zwischen Nachverdichterstufe und Reaktoreinlass mit einer, durch einen Schrittmotor gesteuerten, Spindelpresse [12]. Diese Anordnung stellt zwar deutlich höhere Anforderungen an die Regelung des Systemdrucks als die Zudosierung des Initiators zwischen der zweiten und dritten Verdichterstufe [13], da schon geringe Druckschwankungen zu Schwankungen der Initiatordosierung und somit zu nicht-stationären Betriebszuständen führen können. Andererseits lässt sich bei dieser Anordnung das System bei einer Störung erheblich schneller in einen „initiatorfreien“ und damit sicherer handzuhabenden Zustand bringen. Dadurch konnte mehrfach der Abbruch der gesamten Messreihe verhindert werden. Außerdem kann so gewährleistet werden, dass keine, durch die im Nachverdichter freigesetzte Wärme, induzierte thermische Zersetzung des Initiators eintritt. Dies ist zum einen für die kinetische Auswertung der Experimente von fundamentaler Bedeutung, dient aber zum anderen auch dem Schutz der kostenintensiven Verdichteranlage.

\subsection{Auswahl der Reaktionsbedingungen}

Die im Experiment zugänglichen Reaktionsbedingungen sind durch folgende Punkte gegeben: 
- Apparative Druck- und Temperaturgrenzen: der Hochdruckautoklav ist für Temperaturen von maximal $300{ }^{\circ} \mathrm{C}$ und Drücke bis 3000 bar ausgelegt. Der Maximaldruck der Nachverdichterstufe ist auf 2500 bar begrenzt und legt somit den maximal zugänglichen Druck fest.

- Phasenverhalten im System Ethen/Polyethylen: Der Trübungspunkt, das ist der Punkt, an dem eine Phasentrennung von Polymerisat und Monomer auftritt, hängt vom Druck, der Temperatur, sowie der Molmasse des Polymers ab. Es konnte gezeigt werden [15], dass das System Ethen/Polyethylen bei Temperaturen oberhalb von $130{ }^{\circ} \mathrm{C}$ und einem Zahlenmittel des Molekulargewichts von $10^{4} \mathrm{~g} \cdot \mathrm{mol}^{-1}$ bei ca. 1600 bar homogen ist. Die in dieser Arbeit durchgeführten Experimente wurden daher alle bei 2000 bar durchgeführt. Zusätzlich wurde in allen Experimenten ein Molmassenregler zugesetzt, um die Entstehung hoher Molmassen zu verhindern. Die in einem typischen Experiment bei einem Stoffmengenanteil des Propionaldehyds im Einlassstrom von $f_{\mathrm{PA}}=3 \cdot 10^{-3}$ erhaltenen Zahlenmittel des Molekulargewichts $M_{\mathrm{n}}$ lagen zwischen 10000 und $20000 \mathrm{~g} \cdot \mathrm{mol}^{-1}$. Das Kapillarsystem, das dem Autoklaven nachgeschaltet ist, wurde auf $140{ }^{\circ} \mathrm{C}$ geheizt, um eine Phasentrennung zu verhindern. Somit ist die untere Temperatur des Reaktors auf $140{ }^{\circ} \mathrm{C}$ begrenzt.

- Spontane Zersetzung des Monomers: Auch wenn die Selbst-Initiierung der Polymerisation durch das Monomer Ethen bei der durch die apparativ gegebene Temperaturgrenze von $300{ }^{\circ} \mathrm{C}$ noch relativ langsam verläuft [16], nimmt mit zunehmender Temperatur die Wahrscheinlichkeit einer thermischen Zersetzung des Ethens deutlich zu, die eine Kontrolle des Reaktors unmöglich macht.

- Zerfallsgeschwindigkeit der jeweils untersuchten Initiatoren: Die Reaktionstemperaturen werden so gewählt, dass der Zerfallsgeschwindigkeitskoeffizient des Initiators, $k_{\mathrm{obs}}$, im Bereich von ca. $10^{-3}$ bis $1 \mathrm{~s}^{-1}$ liegt.

- Initiatorkonzentration: Die Stabilität stationärer Betriebsbedingungen nimmt mit zunehmendem Umsatz deutlich ab. Für Monomer-Umsätzen oberhalb $10 \%$ sind Experimente zunehmend schwieriger durchzuführen. Die Initiatorkonzentration wurde daher so gewählt, dass der erzielbare Monomer-Umsatz bei der optimalen Einsatztemperatur eines Initiators möglichst nicht über $10 \%$ liegt. Andererseits sollte der 
durch den Initiator erzielte Umsatz deutlich über dem thermischen Umsatz liegen. Die Initiatorkonzentrationen für die Untersuchungen der organischen Peroxide lagen somit im Bereich zwischen $2.6 \cdot 10^{-6}$ und $2.3 \cdot 10^{-5} \mathrm{~mol} \cdot \mathrm{L}^{-1}$. Je niedriger die optimale Einsatztemperatur eines Initiators liegt, desto höher wurde die Initiatorkonzentration gewählt.

- Massenstrom Ethen: Der Massenstrom des Ethens wurde mit $706 \mathrm{~g} \cdot \mathrm{h}^{-1}$ so gewählt, dass die resultierenden Verweilzeiten mit ca. $140 \mathrm{~s}$ im technisch relevanten Bereich liegen. Bei höheren Massenströmen wurde gezeigt, dass die manuelle Druckregulierung über das Feinventil deutlich schwieriger ist und es daher vermehrt zu starken Druckschwankungen kam, die zu instationären Betriebsbedingungen führen [7, 8].

- Initiatordosierung: Die Vorschubgeschwindigkeit der motorangetriebenen, getakteten Spindelpresse zur Initiatordosierung wurde so gewählt, dass sich eine gesamte Messreihe mit nur einer Füllung der Spindelpresse durchführen lässt.

- Rührergeschwindigkeit: Die Geschwindigkeit des Rührantriebs wurde mit ca. $1000 \mathrm{U} \cdot \mathrm{min}^{-1}$ so hoch wie möglich gewählt, um eine nahezu ideale Vermischung der Reaktionsmischung im Rührkessel zu gewährleisten.

\subsection{Durchführung eines typischen Experiments}

\subsubsection{Vorbereitung einer Messung}

Vor jedem Experiment werden der Reaktor und alle nachfolgenden Komponenten der Anlage zerlegt und bei Temperaturen bis $120^{\circ} \mathrm{C}$ gründlich mit Isododekan gereinigt und anschließend mit Aceton gespült. Am Vortag des Experiments wird die Anlage nach einer Dichtigkeitsprüfung mehrfach mit Ethen gespült und mindestens zwölf Stunden bei 2000 bar mit Ethen gefüllt stehengelassen. Hierbei sollen sich Sauerstoffspuren und andere Verunreinigungen im überkritischen Ethen lösen und anschließend mit diesem ausgetragen werden. Die Dichtigkeit der optischen Hochdruckzelle wird vor jedem Experiment zusätzlich getestet, da eine Undichtigkeit der Zelle, die während des Experiments im Probenraum des FT-IR-Spektrometers justiert ist, zu einer extremen Gefahrensituation führt. Als Lichtquelle wird ein weißglühender Globarstift verwendet, der im Falle einer Leckage ein Ethen-LuftGemisch zur Zündung bringen kann. Aus diesem Grund wird zusätzlich zur 
Dichtigkeitsprüfung der Probenraum des Spektrometers mit technischem $\mathrm{N}_{2}$ gespült, um bei einer Undichtigkeit das Entstehen eines zündfähigen Gemisches zu verhindern.

Aus dem gleichen Grund wird vor Beginn und zum Ende eines Experiments auch das Abluftrohrsystem mit technischem $\mathrm{N}_{2}$ gespült.

\subsubsection{Durchführung einer Messung}

Direkt vor dem Experiment wird der Druck in der Anlage auf 50 bar entspannt und die Thermostatisierung der Dosiersysteme angeschaltet. Alle heizbaren Komponenten der Anlage werden auf Solltemperatur gebracht. Die Temperatur des Reaktors und der nachgeschalteten Komponenten wird mit $140{ }^{\circ} \mathrm{C}$ so gewählt, dass noch keine Reaktion stattfindet. Wenn die optische Hochdruckzelle ihre Solltemperatur erreicht hat, wird der Druck auf 1 bar entspannt und ein Referenzspektrum der Zelle aufgenommen. Nun wird das Ethen bei geöffnetem Ventil des Masseflussreglers bis auf 2000 bar verdichtet. Bei ca. 1000 bar wird das Regelverhalten des Feinventils geprüft und bei ca. 1400 bar werden der Rührer und die Propionaldehyd-Dosierung (4.5 bis $5.0 \mathrm{~g} \cdot \mathrm{h}^{-1}$ entsprechend einer Förderleistung von ca. $6 \mathrm{~mL} \cdot \mathrm{h}^{-1}$ ) eingeschaltet. Anschließend wird der Fluss mit Hilfe des Massenflussreglers eingestellt. Der gewählte Ethen-Durchsatz beträgt für die Mehrzahl der Experimente $706 \mathrm{~g} \cdot \mathrm{h}^{-1}$. Der Reaktionsdruck wird manuell mit Hilfe des Feinventils reguliert. Während des Betriebs registriert das FT-IR-Spektrometer automatisch im vorgegeben Abstand von 2 min Spektren, wobei für jedes Spektrum 100 Interferogramme co-addiert werden. Für die Aufnahme der Spektren werden folgende optische Komponenten verwendet: Globar Lichtquelle, Silicium-beschichteter Calciumfluorid-Strahlteiler und ein mit flüssigem Stickstoff gekühlter MCT-Detektor. Über die FT-IR-Spektren wird der Umsatz, sowie die Stationarität des Systems kontrolliert. Wenn stationäre Konzentrationsverhältnisse erreicht sind, wird der Reaktor auf die gewünschte Reaktionstemperatur gebracht. Nachdem sich wieder ein stabiler Betriebszustand eingestellt hat, wird ca. acht bis zehn Verweilzeiten gewartet, bevor innerhalb eines definierten Zeitraums eine Polymerprobe in der Abscheideeinheit entnommen wird. Die Masse der Probe wird mittels einer Analysenwaage (Sartorius, Göttingen) bestimmt und daraus der Monomer-Umsatz berechnet. Es werden mindestens zwei Proben bei konstantem Umsatz genommen. Nachdem auf diese Weise der thermische Grundumsatz bestimmt worden ist, wird die Initiatorzudosierung eingeschaltet. Nach Erreichen stationärer Polymerisationsbedingungen werden wiederum mindestens zwei Proben genommen. Danach wird die Initiatordosierung ausgeschaltet und der Reaktor auf die nächste Temperatur aufgeheizt. Sobald das System wieder stationär ist, werden wieder 
thermischer Grundumsatz und Umsatz durch Initiator bestimmt. Die Temperatur wird in $10{ }^{\circ} \mathrm{C}$-Schritten solange erhöht, bis der Umsatz wieder abnimmt.

Die Initiatorkonzentration lag bei $4 \cdot 10^{-6}$ und $1 \cdot 10^{-5} \mathrm{~mol} \cdot \mathrm{L}^{-1} \mathrm{im}$ Reaktoreinlassstrom. Dies führt zu einem maximal erzielbaren Umsatz im Bereich 6 bis 12 \%.

\subsubsection{Beendigung des Experiments}

Das Experiment wird wie folgt beendet:

(1) Die Initiatordosierung wird ausgestellt.

(2) Sobald stationäre Betriebsbedingungen herrschen, wird die Propionaldehyd-Dosierung abgestellt und der Massenflussregler auf maximale Förderleistung gestellt.

(3) Sobald eine Abnahme der Propionaldehydkonzentration in den FT-IR-Spektren erkennbar ist, werden die Verdichter auf Bypass-Förderung umgestellt und die gesamte Anlage wird über das Feinventil auf Normaldruck entspannt. Daraufhin werden die Verdichter und alle Heizungen abgeschaltet und die Dosiereinheiten mit $n$-Heptan gespült.

\subsection{Fehlerbetrachtung}

Die Temperatur im Reaktor wurde über das Thermoelement im Reaktormantel geregelt. Für die Auswertung werden die Temperaturen der an unterschiedlichen Positionen in die Reaktionsmischung eintauchenden Thermoelemente verwendet. Die Anzeigegenauigkeit dieser Messstellen beträgt $\pm 1^{\circ} \mathrm{C}$.

Der Druck im Reaktionssystem konnte aufgrund der verwendeten Messgeräte auf \pm 6 bar genau bestimmt werden. Die manuelle Druckregelung mittels des Feinventils ließ sich mit einer Genauigkeit von \pm 15 bar durchführen.

Die Förderleistung der HPLC-Pumpe (Knauer) zur Propionaldehyddosierung wurde während des gesamten Messtages mittels einer Waage kontrolliert und konnte auf $\pm 0.05 \mathrm{~g} \cdot \mathrm{h}^{-1}$ bestimmt werden.

Die Umsatzbestimmung erfolgt mittels einer Analysenwaage (Sartorius) und Stoppuhr. Die Unsicherheit der Massenbestimmung des Polymerisats beträgt 0.02 g. Die Unsicherheit der 
Zeitmessung beträgt $2 \mathrm{~s}$. Dies führt bei einer Probennahme über 5 min und einer Polymermasse von $5 \mathrm{~g}$ bei einem Ethenmassenfluss von $706 \mathrm{~g} \cdot \mathrm{h}^{-1} \mathrm{zu}$ einem relativen Fehler der Umsatzbestimmung von ca. 3 \%.

Bei der Bestimmung der Initiatorverbrauchskurven für die Peroxide wurde die Manteltemperatur in der Regel in $10^{\circ} \mathrm{C}$-Schritten erhöht. Dies entspricht in etwa der Änderung der Reaktorinnentemperatur. Somit lässt sich die Bestimmung der optimalen Temperatur mit einer Genauigkeit von $\pm 5^{\circ} \mathrm{C}$ vornehmen.

\subsection{Simulationsprogramm PREDICI}

Das in dieser Arbeit genutzte kinetische Modell wurde in das Programm PREDICI (Polyreaction Distributions by Countable System Integration), entwickelt von M. Wulkow,[17, 18] implementiert und zur Modellierung der Konzentrationen von Zersetzungsprodukten verwendet, die beim thermischen Zerfall von tert-Alkylperoxyester entstehen. Das Programm nutzt einen diskreten hp-Algorithmus, um komplexe Systeme von Differentialgleichungen zu lösen. Ein integrierter Interpreter erlaubt die mathematische Beschreibung individueller Zusammenhänge von Geschwindigkeitskonstanten, Konzentrationen und Umsätzen der beteiligten Spezies. 


\subsection{Literaturverzeichnis für Kapitel 3}

[1] K. Nozaki, P. D. Bartlett, J. Am. Chem. Soc. 68 (1946) 1686

[2] P. D. Bartlett, R. Altschul, J. Am. Chem. Soc. 67 (1945) 812

[3] J. Barton, E. Borsig, Complexes in Free Radical Polymerization, in: Polymer Science Library 6, A. D. Jenkins, ed. Elsevier, Amsterdam (1988)

[4] M. S. Kharasch, A. Fono, J. Org. Chem. 24 (1959) 606

[5] C. Hinton, Dissertation, Göttingen (1995)

[6] S. Klingbeil, Dissertation, Göttingen (1995)

[7] P. Becker, Dissertation, Göttingen (2000)

[8] J. Sandmann, Dissertation, Göttingen (2000)

[9] M. Buback, M. Busch, K. Lovis, F.-O. Mähling, Macromol. Chem. Phys. 197 (1996) 303

[10] M. Buback, M. Busch, K. Lovis, F.-O. Mähling, Chem. Ing. Tech. 67 (1995) 1652

[11] M. Busch, Dissertation, Göttingen (1993)

[12] F.-O. Mähling, Dissertation, Göttingen (1994)

[13] L. Wittkowski, Dissertation, Göttingen (1998)

[14] K. Lovis, Dissertation, Göttingen (1995)

[15] F. Becker, M. Buback, H. Latz, G. Sadowski, F. Tumakaka, Fluid Phase Equilibria, 215 (2004) 263

[16] M. Buback, Z. Naturforsch. 39a (1984) 399

[17] P. Deufelhardt, M. Wulkow, Konrad Zuse Zentrum, Berlin, Preprint SC94-22 (1994)

[18] "Predici - the software package for realizing polymerization kinetics", proceedings of the $8^{\text {th }}$ conference of the European Consortium of Mathematics in Industry 1994, Springer, Berlin 1995 


\section{Kapitel 4}

\section{Untersuchung der Initiatoreffektivität organischer Peroxide in der radikalischen Hochdruckpolymerisation von Ethen}

\subsection{Einleitung}

Organische Peroxide werden in der radikalischen Polymerisation als Radikalquelle eingesetzt. Ein Maß der Güte eines Initiators ist die Initiatoreffektivität $f$. Sie ist definiert als der auf die Anzahl der entstehenden Primärradikale bezogene Anteil von Radikalen, der eine wachsende Kette startet.

$$
f=\frac{\text { Anteil der durch Initiatorradikale gestarteten Ketten }}{\text { Anzahl der entstandenen Primärradikale }}
$$

Für die Modellierung der kinetischen Prozesse und in der technischen Anwendung der radikalischen Polymerisation ist die Initiatoreffektivität eine wichtige Größe.

In der Literatur gibt es neben den Arbeiten von BecKeR [1, 2] und SANDMAnN [2, 3] wenige Daten für die Initiatoreffektivität von Peroxiden in der Hochdruck-Polymerisation von Ethen [4].

Es wurden allerdings andere Größen eingeführt, um aus initiatorspezifischen Umsatzmessungen verschiedene Initiatoren $\mathrm{zu}$ vergleichen. So wurde der spezifische Initiatorverbrauch, der angibt wie viel g oder mol eines Initiators zur Herstellung einer bestimmten Menge Polymer benötigt wird [4, 5], oder die Initiatorproduktivität eingeführt, die die Stoffmenge umgesetzten Monomers pro Stoffmenge Initiator angibt [6]. Aus diesen Daten kann nur bei gleichen experimentellen Bedingungen eine relative Aussage über die Initiierungseffektivität gemacht werden. Es besteht jedoch keine Möglichkeit, Daten von verschiedenen Autoren bzw. von unterschiedlichen Apparaturen zu vergleichen. Ein weiterer Nachteil dieser Größen ist, dass der unvollständige Initiatorzerfall nicht berücksichtigt wird, der unter Umständen bei einigen Reaktionsbedingungen auftreten kann. Diese Nachteile bestehen hingegen für die Größe der Initiatoreffektivität $f$ nicht. 
In diesem Kapitel der Arbeit soll die Initiatoreffektivität von diversen Peroxiden in der Hochdruckpolymerisation von Ethen in einem kontinuierlich betriebenen Rührkessel mit idealer Rückvermischung (CSTR = continuous stirred tank reactor) bestimmt werden.

\subsection{Theoretische Grundlagen}

Im folgenden Abschnitt werden kurz die theoretischen Grundlagen der idealen HomoPolymerisation und des kontinuierlich betriebenen Rührkessels mit idealer Rückvermischung, dargestellt. Für weitere Details siehe [1, 2, 3].

Das kinetische Schema der Homo-Polymerisation von Ethen kann wie folgt vereinfacht dargestellt werden:

1. Kettenstart: $\quad \mathrm{INI} \stackrel{k_{\mathrm{obs}}}{\longrightarrow} 2 \cdot f \cdot \mathrm{R}_{0}$

2. Kettenwachstum: $\quad \mathrm{R}_{\mathrm{i}}+\mathrm{E} \stackrel{k_{\mathrm{p}}}{\longrightarrow} \mathrm{R}_{\mathrm{i}+1}$

3. Kettenübertragung: $\quad \mathrm{R}_{\mathrm{i}}+\mathrm{CTA} \stackrel{k_{\text {U, СTA }}}{\longrightarrow} \mathrm{P}_{\mathrm{i}}+\mathrm{R}_{0}$

4. Kettenabbruch: $\quad \mathrm{R}_{\mathrm{i}}+\mathrm{R}_{\mathrm{j}} \stackrel{k_{\mathrm{t}, \text { komb }}}{\longrightarrow} \mathrm{P}_{\mathrm{i}+\mathrm{j}}$

$$
\mathrm{R}_{\mathrm{i}}+\mathrm{R}_{\mathrm{j}} \stackrel{k_{\mathrm{t} \text { disp }}}{\longrightarrow} \mathrm{P}_{\mathrm{i}}+\mathrm{P}_{\mathrm{j}}
$$

mit

INI: Initiator

f: $\quad$ Initiatoreffektivität

$\mathrm{R}_{0}$ : Radikal der Kettenlänge 0

$\mathrm{R}_{\mathrm{i}}$ : Radikal der Kettenlänge $\mathrm{i}$

E: Ethen

CTA:Kettenübertragungsagens

$\mathrm{P}_{\mathrm{i}}$ : $\quad$ Polymer der Kettenlänge i $k_{\text {obs: }} \quad$ Zerfallsgeschwindigkeitskonstante von INI

$k_{\mathrm{p}}$ : Wachstumsgeschwindigkeitskonstante

$k_{\text {Ü, СтА }}$ : Geschwindigkeitskonstante der Übertragung zum CTA

$k_{\mathrm{t}, \text { komb}}$ : Geschwindigkeitskonstante des Kettenabbruchs durch Kombination

$k_{\mathrm{t}, \text { disp: }}$ Geschwindigkeitskonstante des Kettenabbruchs durch Disproportionierung

Der Gesamtumsatz an Monomer wird durch die Reaktionen 1, 2 und 4 bestimmt. Demnach ist die Kenntnis der kinetischen Koeffizienten des Initiatorzerfalls $k_{\mathrm{obs}}$, des Kettenwachstums $k_{\mathrm{p}}$ 
und des Kettenabbruchs $k_{\mathrm{t}}$ notwendig, um aus Umsatzdaten auf die Initiatoreffektivität zu schließen. Für die Kettenwachstumsreaktion werden die temperatur-, druck- und umsatzabhängigen Daten von SCHWEER [7] verwendet. Die temperatur- und druckabhängigen Geschwindigkeitskonstanten für die Kettenabbruchreaktion werden ebenfalls von SCHWEER übernommen, doch ist die Umsatzabhängigkeit bei kleinen Ethen-Umsätzen überschätzt. Diese ist allerdings in der modifizierten, in Referenz [2] beschriebenen Form, korrigiert.

Es wurden folgende Geschwindigkeitsgesetze angenommen:

$$
\begin{aligned}
& \frac{\mathrm{d} c_{\mathrm{INI}}}{\mathrm{d} t}=-k_{\mathrm{obs}} \cdot c_{\mathrm{INI}} \\
& \frac{\mathrm{d} c_{\mathrm{Ri}}}{\mathrm{d} t}=2 \cdot f \cdot k_{\mathrm{obs}} \cdot c_{\mathrm{INI}}-2 \cdot k_{\mathrm{t}} \cdot c_{\mathrm{Ri}}^{2} \\
& \frac{\mathrm{d} c_{\mathrm{E}}}{\mathrm{d} t}=-k_{\mathrm{p}} \cdot c_{\mathrm{E}} \cdot c_{\mathrm{Ri}}
\end{aligned}
$$

mit

$C_{\text {INI }}: \quad$ stationäre Konzentration an Initiatormolekülen

$c_{\mathrm{Ri}}$ : $\quad$ stationäre Konzentration an Radikalen der Kettenlänge i

$c_{\mathrm{E}}: \quad$ Ethenkonzentration

$t: \quad$ Zeit

Die Bilanz einer beliebigen Teilchenart X in einem kontinuierlich betriebenen Rührkessel mit idealer Rückvermischung im stationären Zustand wird durch folgende Gleichung beschrieben [8]:

$$
\frac{\mathrm{d} c_{\mathrm{X}}}{\mathrm{d} t}=0=c_{\mathrm{X}, 0} \cdot \frac{\rho_{\mathrm{A}}}{\rho_{\mathrm{E}}}-c_{\mathrm{X}}+\tau \cdot \sum R_{\mathrm{X}, \mathrm{i}}
$$

mit

$c_{\mathrm{X}}: \quad$ Konzentration der Teilchenart X im CSTR

$c_{\mathrm{X}, 0}: \quad$ Konzentration der Teilchenart X im Reaktoreinlassstrom

$\rho_{\mathrm{E}}: \quad$ Dichte am Reaktoreinlass 
$\rho_{\mathrm{A}}: \quad$ Dichte am Reaktorauslass

$\tau$ : $\quad$ hydrodynamische Verweilzeit

$\sum R_{\mathrm{X}, \mathrm{i}}$ : Reaktionsgeschwindigkeit der von $c_{\mathrm{X}}$ abhängigen Teilreaktionen im CSTR

Unter der Voraussetzung, dass die ideale Polymerisationskinetik und Massenbilanz des CSTR gültig sind, lässt sich mit diesem Ansatz nun jede Konzentration einer beliebigen Teilchenart berechnen. Weiterhin wurde eine Gleichung abgeleitet, mit der die Initiatoreffektivität berechnet werden kann [1, 2, 3]. Sie lautet:

$$
f=\left(\frac{\Delta U}{1-\Delta U}\right)^{2} \cdot \frac{k_{\mathrm{t}}}{\left(\tau \cdot k_{\mathrm{p}}\right)^{2}} \cdot \frac{\rho_{\mathrm{E}}}{\rho_{\mathrm{A}} \cdot c_{\mathrm{INI}, 0}} \cdot \frac{1+\tau \cdot k_{\mathrm{obs}}}{k_{\mathrm{obs}}}
$$

wobei $\Delta U$ der Peroxid-induzierte Ethenumsatz ist. Die Bestimmung der Initiatoreffektivität mit Gleichung (4.5) wird im Folgenden Methode 1 bzw. analytische Bestimmung genannt. Zusätzlich wurde die Größe „reduzierter Umsatz“ eingeführt [1, 2, 3]. Sie soll es ermöglichen, Experimente bei verschiedenen Initiatorkonzentrationen zu vergleichen.

$$
\text { Reduzierter (Monomer-)Umsatz } \equiv \frac{\Delta U}{\sqrt{c_{\text {INI, zersetzt }}}}=\Delta U_{\mathrm{r}}=\frac{k_{\mathrm{p}}}{\sqrt{k_{\mathrm{t}}}} \cdot \sqrt{\tau} \cdot \sqrt{f}
$$

$c_{\text {INI, zersetzt }}$ ist die Konzentration an Initiator, der im CSTR zerfällt. Der reduzierte Umsatz wurde aus dem oben aufgeführten kinetischen Schema unter der Annahme abgeleitet, dass das Produkt $k_{\mathrm{p}} \cdot C_{\mathrm{Ri}} \cdot \tau<1$ sein muss. Für die Experimente im Rahmen dieser Arbeit liegt das Produkt $k_{\mathrm{p}} \cdot c_{\mathrm{Ri}} \cdot \tau \approx 0.02$ bis 0.05 . Weiterhin wird angenommen, dass die Dichte im Reaktoreinlass $\rho_{\mathrm{E}}$ und die Dichte am Reaktorauslass $\rho_{\mathrm{A}}$ gleich ist mit: $\rho_{\mathrm{A}} / \rho_{\mathrm{E}} \approx 1$. Bei $200{ }^{\circ} \mathrm{C}$ und $6 \%$ Monomer-Umsatz ist $\rho_{\mathrm{A}} / \rho_{\mathrm{E}}=1.03$. Gleichung (4.6) sollte somit gut anwendbar sein. Aus Gleichung (4.6) wird ersichtlich, dass bei gleicher Verweilzeit der reduzierte Umsatz nur von den kinetischen Daten des Monomers $\left(k_{\mathrm{p}}\right.$ und $k_{\mathrm{t}}$ ) und der Initiatoreffektivität $f$ abhängig ist. Dies eröffnet eine weitere Methode zur Bestimmung von $f$ direkt aus den experimentellen Daten.

Die Temperaturabhängigkeit des reduzierten Umsatzes sollte unter der Annahme einer temperaturunabhängigen Initiatoreffektivität und Verweilzeit im CSTR nach Gleichung (4.6) ausschließlich von der Temperaturabhängigkeit des Terms $k_{\mathrm{p}} / \sqrt{k_{\mathrm{t}}}$ des Monomers (in dieser Arbeit Ethen) bestimmt sein. 


$$
\ln \Delta U_{\mathrm{r}}=\ln \frac{k_{\mathrm{p}}}{\sqrt{k_{\mathrm{t}}}}+\ln \sqrt{\tau}+\ln \sqrt{f}
$$

Eine Auftragung des logarithmierten reduzierten Umsatzes (4.7) gegen die reziproke Temperatur sollte somit eine Gerade mit der Steigung entsprechend der Aktivierungsenergie von $k_{\mathrm{p}} / \sqrt{k_{\mathrm{t}}}$ des Monomers ergeben. Initiatoren unterschiedlicher Effektivität führen in dieser Auftragung nach Gleichung (4.7) zu parallel verschobenen Geraden. Aus der relativen Lage dieser Geraden lässt sich die Initiatoreffektivität ermitteln, wenn eine umsatz- und temperaturunabhängige Effektivität angenommen wird. Für Di-tert-Butylperoxid (DTBP) wurde eine Initiatoreffektivität von 1 gefunden, so dass die Ausgleichsgerade dieser Messwerte als Referenz zur Bestimmung von $f$ dient $[1,2,3]$. Die relative Lage von ln $\Delta U_{\mathrm{r}}$ eines Peroxids in der Arrhenius-Auftragung in Bezug zu dieser Ausgleichsgerade sei

$$
\Delta y=\ln \Delta U_{\mathrm{r}}(\mathrm{DTBP})-\ln \Delta U_{\mathrm{r}}
$$

Dann ist mit Gleichung 4.7

$$
\Delta y=\left[\ln \frac{k_{\mathrm{p}}}{\sqrt{k_{\mathrm{t}}}}+\ln \sqrt{\tau}+\ln \sqrt{f}\right]_{\text {DTBP }}-\left[\ln \frac{k_{\mathrm{p}}}{\sqrt{k_{\mathrm{t}}}}+\ln \sqrt{\tau}+\ln \sqrt{f}\right]
$$

Werden die Initiatoren wie in dieser Arbeit bei identischen Verweilzeiten $\tau$ untersucht und wird eine gleichartige Temperaturabhängigkeit der kinetischen Koeffizienten $k_{\mathrm{p}}$ und $k_{\mathrm{t}}$ für diese Reaktionsbedingungen angenommen, so lässt sich Gleichung 4.9 wie folgt vereinfachen:

$$
\Delta y=\ln \sqrt{f_{\text {DTBP }}}-\ln \sqrt{f}=\ln \sqrt{\frac{f_{\text {DTBP }}}{f}}
$$

Wird Gleichung 4.10 nach $f$ aufgelöst, wird eine weitere Methode zur Bestimmung der Initiatoreffektivität erhalten, die im Folgenden Methode 2 bzw. Relativmethode zu DTBP genannt wird.

$$
f=\frac{f_{\text {DTBP }}}{(\exp (\Delta y))^{2}}
$$




\subsection{Voruntersuchungen}

Es wurden von BECKER [1] und SANDMANN [3] Experimente durchgeführt, um geeignete Messbedingungen $\mathrm{zu}$ finden und $\mathrm{zu}$ zeigen, dass die in Kapitel 4.2. beschriebene Vorgehensweise zulässig ist.

Dazu wurde der Einfluss folgender Parameter überprüft:
i) Einfluss des Kettenübertragungsagens (CTA = chain transfer agent) auf den Initiatorzerfall
ii) Einfluss des CTA auf den Monomer-Umsatz
iii) Einfluss des Initiator-Lösungsmittels $n$-Heptan auf den Monomer-Umsatz
iv) Einfluss der Rührergeschwindigkeit auf den Monomer-Umsatz
v) Einfluss der Verweilzeit auf den Monomer-Umsatz

i) Ein Einfluss des Kettenübertragungsreagenzes auf den Initiatorzerfall konnte nicht festgestellt werden. Eine Mischung aus Peroxid und Kettenübertragungsreagenz (in diesem Fall Propionaldehyd) mit fünffach höherer Konzentration wurde in $n$-Heptan gelöst und die Zerfallskinetik gemessen. Die höhere Konzentration des Propionaldehyds sollte der Tatsache Rechnung tragen, dass auch in der Polymerisation die Konzentration des Propionaldehyds deutlich über der des Initiators liegt. Es konnte kein signifikanter Unterschied der Zersetzungsgeschwindigkeit des Peroxids im Vergleich zur Lösung nur in $n$-Heptan beobachtet werden.

ii) Von BuBACK [9] wurde gezeigt, dass bei den gewählten Versuchsbedingungen Ethen ohne Zugabe von Initiator nur in sehr geringem Ausmaß thermisch induziert spontan polymerisiert. Bei Zugabe von Propionaldehyd wurden signifikant höhere Monomer-Umsätze gemessen. Dieser Monomer-Umsatz wurde als thermischer Grundumsatz bezeichnet. Durch eine Konzentrationsvariation konnte gezeigt werden, dass sich Propionaldehyd wie ein „schlechter Initiator“ verhält. Es kann angenommen werden, dass sich der thermische Grundumsatz, verursacht durch Propionaldehyd, und der durch den Initiator hervorgerufene MonomerUmsatz additiv verhalten. Da an verschiedenen Messtagen unterschiedliche Absolutwerte für den thermischen Grundumsatz erhalten wurden, muss während eines Experiments vor jeder Umsatzbestimmung mit Initiator der thermische Grundumsatz gemessen werden.

iii) Das Peroxid wird in Lösung von $n$-Heptan zur Reaktionsmischung eindosiert. Es wurde gezeigt, dass sich der thermische Grundumsatz durch die Anwesenheit geringer Mengen von $n$-Heptan (Massenfluss $n$-Heptan $=1 \mathrm{~g} \cdot \mathrm{h}^{-1}$ ) nicht signifikant unterscheidet. 
iv) Bei der Polymerisation im CSTR hat die Rührergeschwindigkeit einen großen Einfluss auf den maximal erreichbaren Monomer-Umsatz, da bei zu niedriger Rührergeschwindigkeit der eindosierte Initiator nicht homogen im CSTR verteilt wird. Dies führt zu lokal erhöhten Radikalkonzentrationen, was die Terminierungsreaktion begünstigt und den MonomerUmsatz senkt [5, 10]. Um dies zu überprüfen, wurde der Umsatz in Abhängigkeit der Rührergeschwindigkeit gemessen. Es konnte gezeigt werden, dass oberhalb einer Drehzahl von $750 \mathrm{U} \cdot \mathrm{min}^{-1}$ kein Einfluss auf den Monomer-Umsatz vorhanden ist. Bei nicht ausreichender Vermischung (im Drehzahlbereich unterhalb von $750 \mathrm{U} \cdot \mathrm{min}^{-1}$ ) nimmt der Umsatz kontinuierlich ab. Die weiteren Experimente wurden daher bei maximaler Rührergeschwindigkeit von $1000 \mathrm{U} \cdot \mathrm{min}^{-1}$ durchgeführt, um eine optimale Vermischung zu gewährleisten.

v) Die Verweilzeit im CSTR hat ebenfalls einen Einfluss auf den Monomer-Umsatz und auf die Lage der optimalen Temperatur. Dies ist die Temperatur, bei der der Monomer-Umsatz unter ansonsten gleichen Versuchsbedingungen ein Maximum durchläuft. Mit zunehmender Verweilzeit nimmt der Monomer-Umsatz zu und die optimale Temperatur ab. Dies wurde auf den Initiatorumsatz zurückgeführt, der bei der verwendeten Apparatur bei der optimalen Temperatur um $98 \%$ liegt. Dieser Initiatorumsatz wird bei höheren Verweilzeiten bei niedrigeren Temperaturen erreicht [1, 3].

Eine weitere Erklärung für die Änderung der Temperatur liegt in der experimentellen Vorgehensweise. Die Variation der Verweilzeiten wurde durch unterschiedliche Massenströme bei konstantem Reaktorvolumen erhalten. Dadurch verändert sich das Strömungsverhalten und die Einmischcharakteristik am Reaktoreinlass. Bei höheren Massenflüssen bedeutet dies wiederum eine bessere Vermischung, die wie oben erklärt einen höheren Monomer-Umsatz bewirkt. Dies führt ebenfalls zu einer höheren optimalen Temperatur.

\subsection{Auswertung eines typischen Experiments}

Wie in Kapitel 3 beschrieben, wird im Experiment in der Regel bei konstantem Druck und konstanter Initiatordosierung die Temperaturabhängigkeit des Ethenumsatzes in An- und Abwesenheit des Initiators bestimmt. Aufgrund der Vielzahl relevanter experimenteller Parameter ( $p, T, f_{\mathrm{PA}}, c_{\mathrm{INI}, 0}, \tau, U$ etc.), wurden einige Größen in den Experimenten konstant gehalten, um eine Vergleichbarkeit der einzelnen Messreihen zu gewährleisten. Der 
Reaktionsdruck betrug 2000 bar, außer bei den Druckabhängigkeitsexperimenten, der Ethenmassenstrom lag bei $706 \mathrm{~g} \cdot \mathrm{h}^{-1}$, was einer durchschnittlichen Verweilzeit von ca. $140 \mathrm{~s}$ entspricht. Der Stoffmengenanteil des Propionaldehyds im Reaktoreinlassstrom lag bei 0.0032, das entspricht ca. $0.3 \mathrm{~mol} \%$. Die Initiatordosierung wurde so gewählt, dass die maximal erreichbaren Umsätze möglichst zwischen vier und zehn Prozent lagen. In Abbildung 4.1 ist das Ergebnis eines solchen Experiments mit 1,1,3,3-Tetramethylbutylperoxy-2-ethylhexanoat (TMBPO) als Initiator gezeigt. Die ausgefüllten Kreise entsprechen dem thermischen Grundumsatz. Dieser wurde jeweils nach Erreichen der gewünschten Manteltemperatur direkt vor der nächsten Initiatorzudosierung bestimmt. Sobald nach Beginn der Initiatoreindosierung wieder stationäre Polymerisationsbedingungen vorlagen, wurde der Gesamtethenumsatz mit Initiator (offene Kreise) bestimmt.

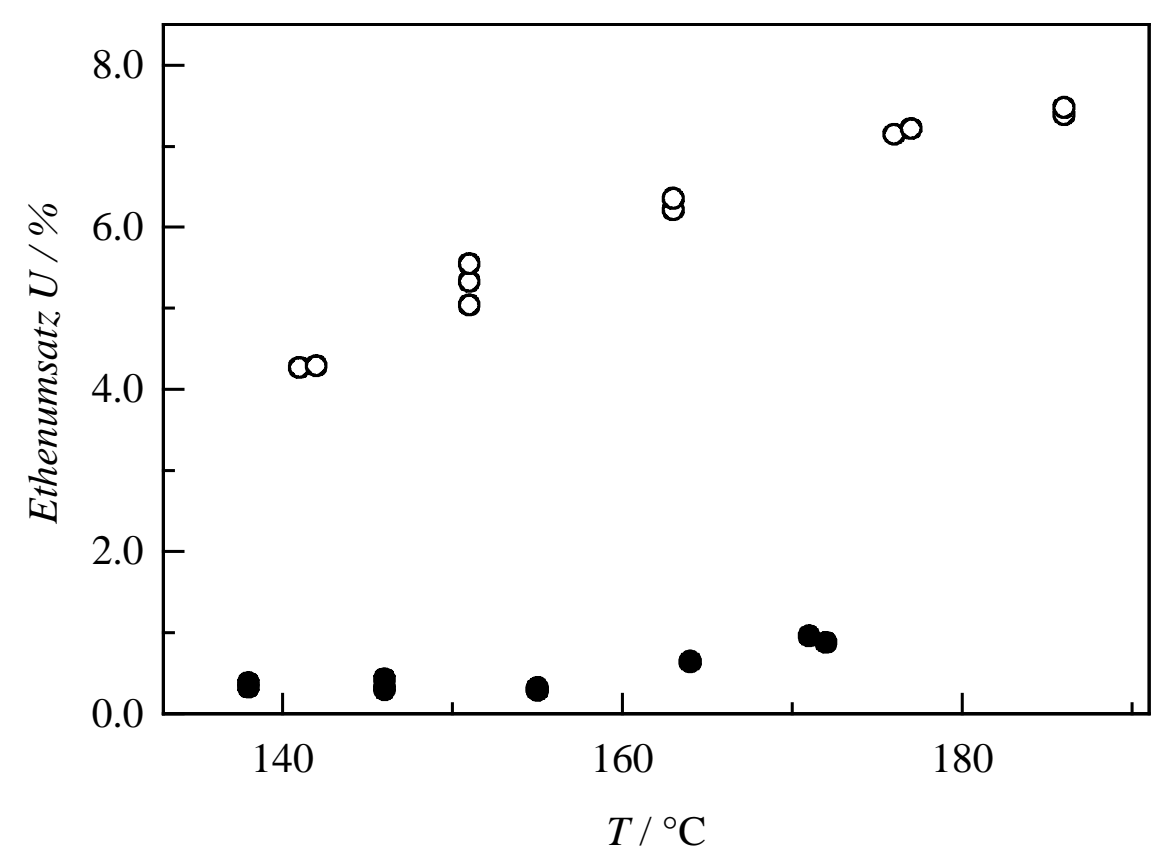

Abb. 4.1: Temperaturabhängigkeit des Gesamtethen- und des Grundumsatzes in der EthenHochdruckpolymerisation bei 2000 bar mit TMBPO als Initiator. $c(\text { TMBPO })_{0}=1.4 \cdot 10^{-5} \mathrm{~mol} \cdot \mathrm{L}^{-1} ; f_{P A}=0.0032 ; m_{E}=706 \mathrm{~g} \cdot \mathrm{h}^{-1}$.

Abbildung 4.2 zeigt den um den thermischen Grundumsatz bereinigten Umsatz, im nachfolgenden Text Peroxid-induzierter Ethenumsatz $\Delta U$ genannt, für das in Abbildung 4.1 gezeigte Experiment, sowie für ein weiteres Experiment mit halbierter Initiatorkonzentration. Um $\Delta U$ zu erhalten, wird der Grundumsatz vom Gesamt-Ethenumsatz abgezogen. Beide werden bei der gleichen Manteltemperatur gemessen. 


$$
\Delta U=\text { Gesamt-Ethenumsatz }- \text { Grundumsatz }
$$

Die maximalen Peroxid-induzierten Umsätze sind 4.15 bzw. 6.55 \%. Das Verhältnis der beiden maximalen Umsätze entspricht ungefähr $\sqrt{2}$, was nach der idealen Polymerisationskinetik bei der Verdoppelung der Initiatorkonzentration erwartet wird. Als typischer UmsatzTemperatur-Verlauf wird (auch nach der Korrektur) ein Anstieg des Peroxid-induzierten Umsatzes mit steigender Temperatur gefunden. Dieser durchläuft bei der im folgenden $T_{\mathrm{opt}}$ genannten Temperatur ein Maximum und fällt zu höheren Temperaturen wieder ab.

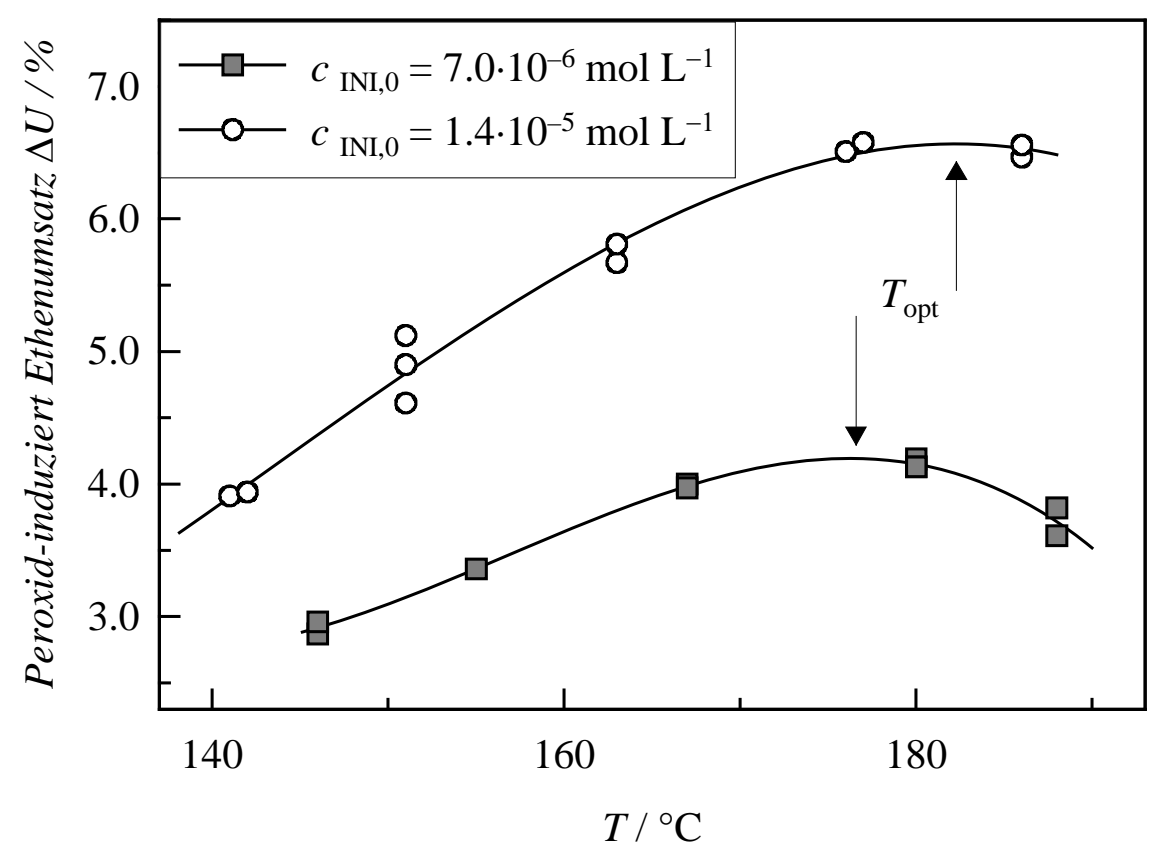

Abb. 4.2: Temperaturabhängigkeiten des Peroxid-induzierten Ethenumsatzes für EthenHomopolymerisationen mit TMBPO als Initiator. Die optimalen Temperaturen der zwei Experimente sind durch Pfeile gekennzeichnet; gefüllte Quadrate $c(\mathrm{TMBPO})_{0}=7.0 \cdot 10^{-6} \mathrm{~mol} \cdot \mathrm{L}^{-1}$; offene Kreise $\mathrm{C}(\mathrm{TMBPO})_{0}=1.4 \cdot 10^{-5} \mathrm{~mol} \cdot \mathrm{L}^{-1}$; $p=2000 \mathrm{bar}, m_{E}=706 \mathrm{~g} \cdot \mathrm{h}^{-1}$.

Dieser Verlauf lässt sich folgendermaßen erklären: Mit steigender Temperatur nimmt die Zerfallsgeschwindigkeit des Initiators zu. Dadurch erhöht sich der Initiatorumsatz bei vorgegebener Verweilzeit und damit die Konzentration der Radikale. Somit wird auch ein Anstieg des erzielbaren Umsatzes mit steigender Temperatur erwartet. Außerdem erhöht sich auch die Polymerisationsgeschwindigkeit, da auch $\left(k_{\mathrm{p}} / k_{\mathrm{t}}^{0.5}\right)$ mit der Temperatur ansteigt, d.h. der pro erzeugtem Radikal erzielbare Umsatz nimmt mit steigender Temperatur zu. Nähert sich die Temperatur nun $T_{\mathrm{opt}}$, so erfolgt der Initiatorzerfall schneller als eine vollständige Einmischung des Initiators erreicht werden kann. Daher entsteht am Reaktoreinlass lokal eine 
höhere Radikalkonzentration und die Wahrscheinlichkeit des Kettenabbruchs durch Kombination oder Disproportionierung zweier Radikale nimmt zu. Der Umsatz stagniert und geht bei noch höheren Temperaturen, und damit noch schnellerem Initiatorzerfall, sogar zurück. Wie Abbildung 4.2 zeigt, ist die Lage der optimalen Temperatur ( $T_{\text {opt }}=177$ bzw. $\left.182{ }^{\circ} \mathrm{C}\right)$ innerhalb der Messgenauigkeit $\left( \pm 5^{\circ} \mathrm{C}\right)$ nicht von der Initiatorkonzentration abhängig.

Aus den so experimentell bestimmten Umsatzdaten lassen sich die Größen spezifischer Initiatorverbrauch ID (Gleichung 4.13) und Initiatorproduktivität IP (Gleichung 4.14) berechnen. Die entsprechende Auftragung ist in Abbildung 4.3 gezeigt.

$$
I D=\frac{\text { eingesetzte Initiatormenge }[\mathrm{mol}]}{\text { Polymerisatmenge }[\mathrm{kg}]}
$$

$$
I P=\frac{\text { umgesetzte Monomermenge }[\mathrm{mol}]}{\text { eingesetzte Initiatormenge }[\mathrm{mol}]}
$$

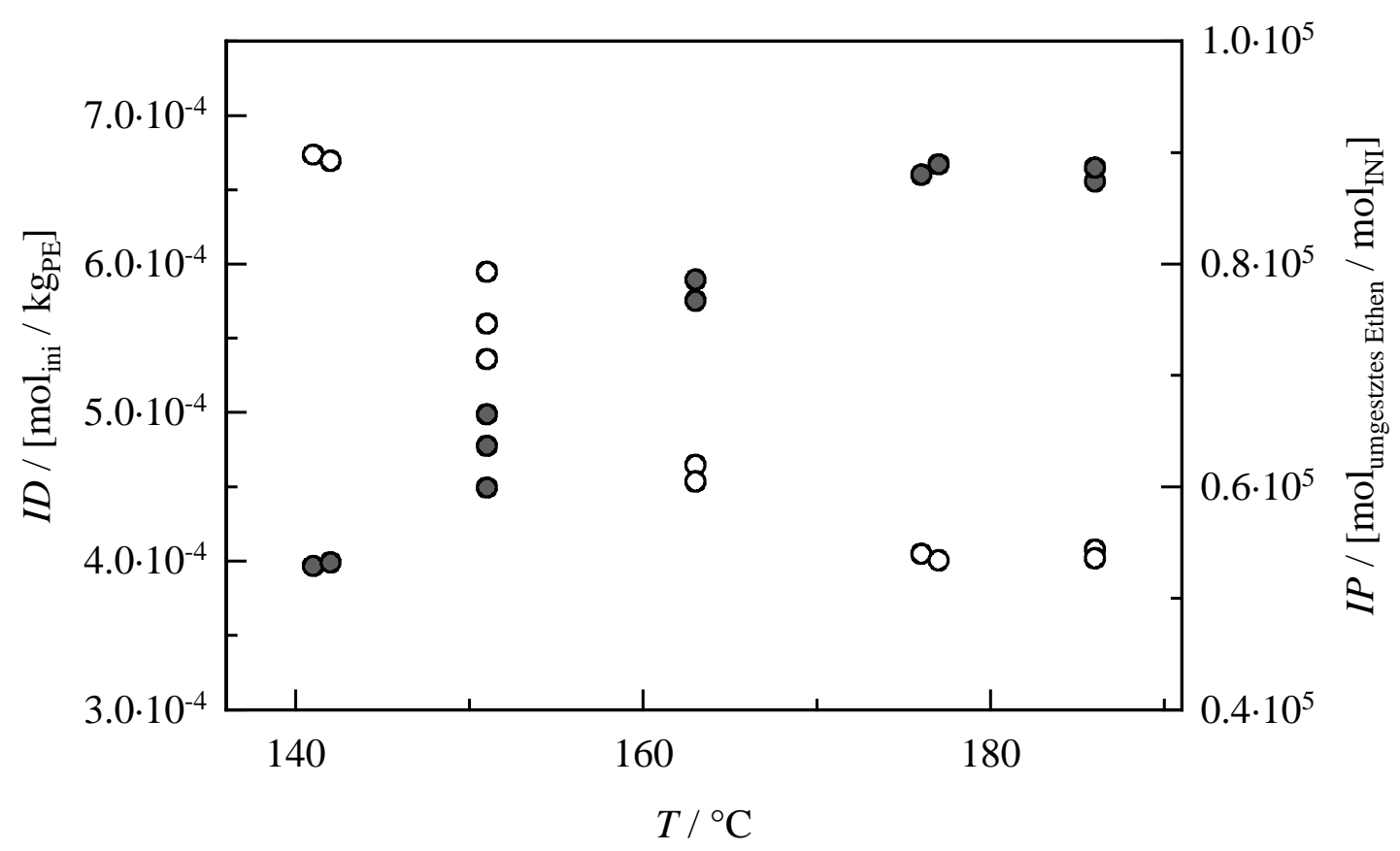

Abb. 4.3: Spezifischer Initiatorverbrauch, ID (offene Symbole), Initiatorproduktivität, IP (gefüllte Symbole), des in Abbildung 4.1 dargestellten Experiments.

Die in Abbildung 4.3 dargestellten Größen spiegeln qualitativ das Umsatz-Temperatur-Profil aus Abbildung 4.2 wider. Der spezifische Initiatorverbrauch durchläuft bei $T_{\text {opt }}$ ein Minimum, 
die Initiatorproduktivität dagegen ein Maximum. Für $T=T_{\mathrm{opt}}$ wird also die geringste Menge Initiator benötigt, um eine bestimmte Menge Polymer herzustellen, bzw. die Anzahl der pro zersetztem Initiatormolekül umgesetzten Monomermoleküle ist maximal. Zur Berechnung beider Größen wird jedoch nicht berücksichtigt, dass bei niedrigeren Temperaturen kein vollständiger Initiatorumsatz erzielt wird. Dieser Aspekt spielt allerdings auch bei der Beurteilung aus anwendungstechnischer Sicht eine untergeordnete Rolle, da der nicht umgesetzte Initiator nicht weiter verwendet werden kann. Zudem ist dies keine sinnvolle technische Reaktionsbedingung, da der Initiator beim Recyceln des unverbrauchten Ethens akkumuliert.

Um auch Messreihen, die mit unterschiedlichen Initiatorkonzentrationen durchgeführt wurden, miteinander vergleichen zu können, wurde in Kapitel 4.2 der "reduzierte Umsatz" eingeführt. Dieser Reduktion liegt die Annahme zugrunde, dass bei einer Polymerisation in einem CSTR der Umsatz näherungsweise proportional zur Wurzel der Initiatorkonzentration ist. Außerdem wird berücksichtigt, dass bei niedrigeren Temperaturen der Initiator nicht zu 100 \% zerfallen ist. Die Definition für den reduzierten Umsatz (siehe Kapitel 4.2) lautet:

$$
\text { Reduzierter (Monomer-)Umsatz } \equiv \frac{\Delta U}{\sqrt{C_{\text {INI,zersetzt }}}}
$$

Die Gültigkeit dieses Modells soll am Beispiel von zwei Messreihen des 1,1,3,3-Tetramethylbutylperoxy-2-ethyl-hexanoats (TMBPO) bei unterschiedlichen Initiatorkonzentrationen gezeigt werden. In Abbildung 4.2 sind die zwei erhaltene Umsatz-Temperatur-Profile gezeigt. In Abbildung 4.4 ist für die gleichen Messungen der reduzierte Ethenumsatz als Funktion der Temperatur gezeigt. Im Bereich $T<T_{\text {opt }}$ stimmen die reduzierten Umsätze für die zwei Initiatorkonzentrationen innerhalb der Messgenauigkeit überein. Die stärkere Streuung der Daten nach Erreichen von $T_{\text {opt }}$ kommt dadurch zustande, dass sich die Nichtidealitäten des Systems unterschiedlich auf die einzelnen Messwerte auswirken. 


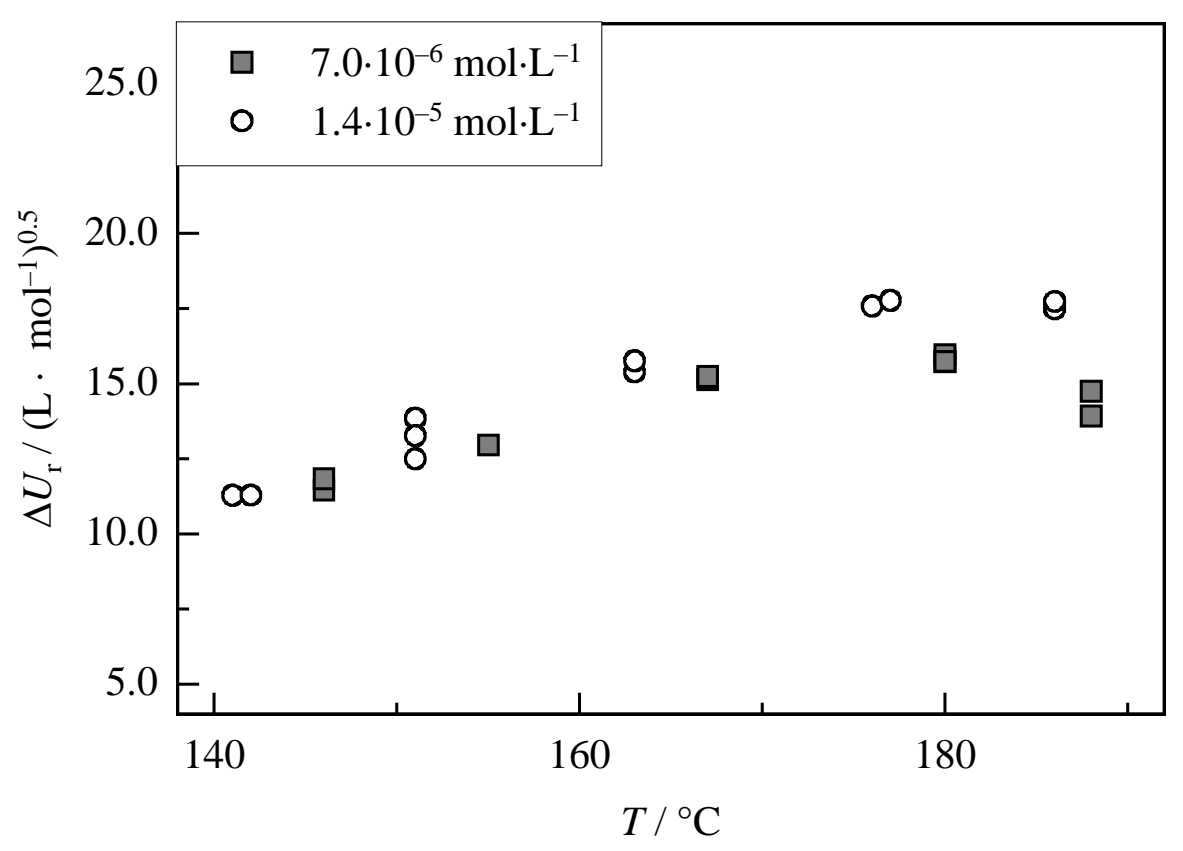

Abb.4.4: Reduzierter Ethenumsatz für die in Abbildung 4.2 gezeigten Experimente; $p=2000$ bar; $m_{E}=706 \mathrm{~g} \cdot h^{-1} ; f_{P A}=0.0032$.

Zur Bestimmung von $c_{\text {INI,zersetzt }}$ muss die verwendete Initiatormenge genau bekannt sein. Im Rahmen dieser Doktorarbeit wurde festgestellt, dass BECKER und SANDMANN eine inadäquate Kalibrierkurve der Spindelpresse zur Eindosierung des Initiators benutzten. In Abbildung 4.5 sind für drei baugleiche Spindelpressen SP I, SP II und SP III der Volumenfluss gegen den Spindelpressenvorschub aufgetragen. Die Kalibrierungen der drei Spindelpressen von HinRICHS/JAuER und MÜller sind identisch. SP I war die Spindelpresse, die BECKER/SANDMANN und HinRICHS/JAUER in ihren Experimenten benutzt haben. Die senkrechte Linie gibt den Spindelpressenvorschub an, bei dem alle Experimente durchgeführt worden sind. 


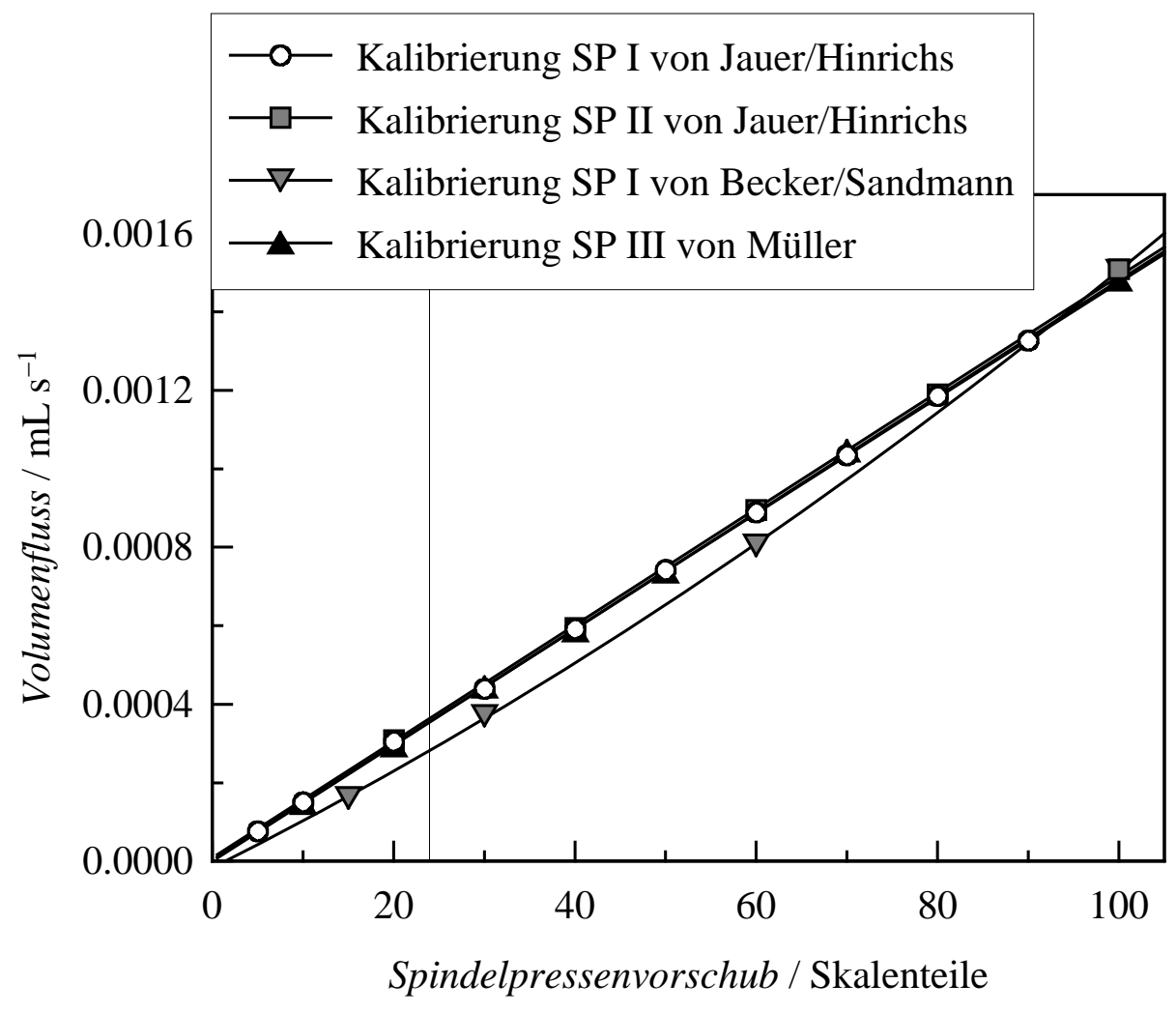

Abb.4.5: Kalibrierkurven von drei baugleichen Spindelpressen durchgeführt zu unterschiedlichen Zeitpunkten. SP I zeigt identischen Volumenfluss mit SP II und SP III, wenn die Kalibrierung von HINRICHS/JAUER verwendet wird. Einzig die Kalibrierung von BECKER/SANDMANN zeigt Abweichungen.

Bei den von Becker/Sandmann durchgeführten Experimenten wurde ein um ca. $20 \%$ geringerer Initiatorvorschub angenommen als der aktuelle Fluss. Dieser Fehler muss nicht zwangsläufig zu größeren Initiatoreffektivitäten führen, wenn die Effektivität relativ zu DTBP bestimmt wurde und die Referenzeffektivität von DTBP korrekt ist. Deshalb ist es wichtig; die Größe der Initiatoreffektivität von DTBP zu überprüfen. Diese Überprüfung wurde mit Gleichung 4.5 durchgeführt. Die erhaltende Initiatoreffektivität ist temperaturunabhängig und nahe eins (Abbildung 4.6), wie bei BECKER und SANDMANN. 


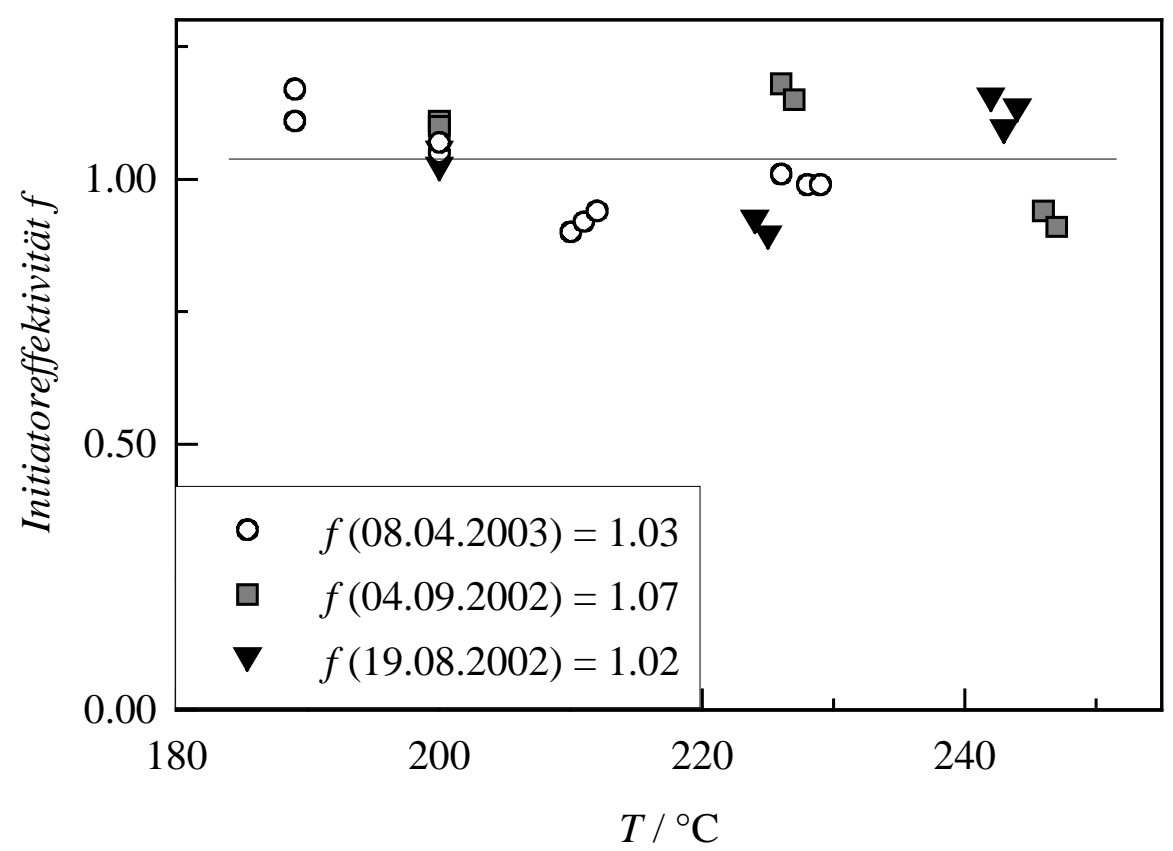

Abb. 4.6: $\quad$ Temperaturabhängigkeit der Initiatoreffektivität für DTBP mit Gleichung 4.5 erhalten. $p=2000$ bar, $m_{E}=706 \mathrm{~g} \mathrm{~h}^{-1} ; c(D T B P)_{0}=2.6 \cdot 10^{-6} \mathrm{~mol} \mathrm{~L}^{-1}$ für alle drei Messungen.

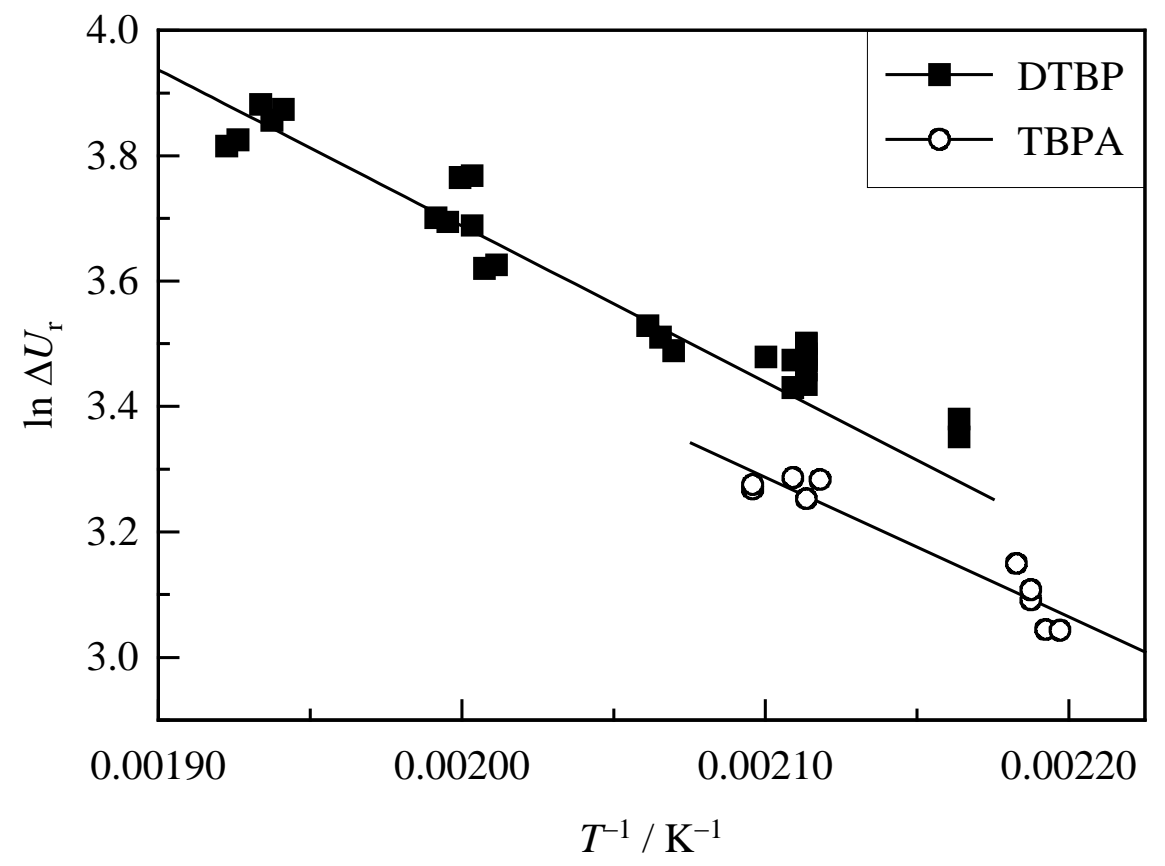

Abb.4.7: Experimentelle Daten für den reduzierten Monomer-Umsatz, erhalten aus mehreren unabhängigen Hochdruckpolymerisations-Experimenten von Ethen mit tert-Butylperoxyacetat (TBPA) oder DTBP als Initiator; $p=2000$ bar, $m_{E}=706 \mathrm{~g} \cdot \mathrm{h}^{-1} ; c(D T B P)_{0}=2.6 \cdot 10^{-6} \mathrm{~mol} \cdot \mathrm{L}^{-1}, c(D T B P)_{0}=5.4 \cdot 10^{-6} \mathrm{~mol} \cdot \mathrm{L}^{-1}$.

Abbildung 4.7 veranschaulicht die Primärdaten - der reduzierte Umsatz in der ArrheniusAuftragung - für Peroxid-induzierte Ethenpolymerisationen. Anhand der Abbildung 4.7 ist zu erkennen, dass die aus verschiedenen, unabhängigen Experimenten erhaltenden Daten sehr 
gut übereinstimmen. Die Reproduzierbarkeit von $f$ wird besser als \pm 0.10 abgeschätzt. Die Daten in Abbildung 4.6 und 4.7 zeigen, dass die Auswertung von $f$ über den reduzierten Monomerumsatz mit der Referenz von $f=1$ für DTBP eine gültige Methode ist. Im nachfolgenden Text sind die Initiatoreffektivitäten aus den Literaturstellen [1, 2, 3, 15, 16], sowie die im Rahmen der Arbeit untersuchten Peroxide relativ zu der, in Abbildung 4.7 gezeigten, Ausgleichsgerade berechnet. Die Punkte für DTBP bei den zwei tiefsten Temperaturen wurden bei der Ausgleichsgerade nicht berücksichtigt, weil die Unsicherheit dieser Punkte im Vergleich zu den restlichen Punkten sehr hoch ist [11].

\subsection{Initiatorcocktails}

Mit der Untersuchung von Initiatorcocktails sollte überprüft werden, ob sich die Initiatoreffektivität eines Peroxids während der Polymerisation in Ethen bei 2000 bar in Anwesenheit eines anderen Peroxids verändert und wie der Einfluss des Initiatorcocktails auf das Ethenumsatz-Temperaturprofil ist. Dafür wurden zwei Experimente durchgeführt. Bei einem wurden Peroxide verwendet, deren optimale Temperaturen weit auseinander lagen (TBPA und TBPP), bei dem anderen wurden Peroxide verwendet, deren optimale Temperaturen sehr ähnlich waren (BTMHP und TBPO). Die Zerfallskinetik wurde in $n$-Heptan in einem weiten Zustandsbereich gemessen [12, 13, 14]. In Abbildung sind die Strukturformeln der verwendeten Peroxide gezeigt.
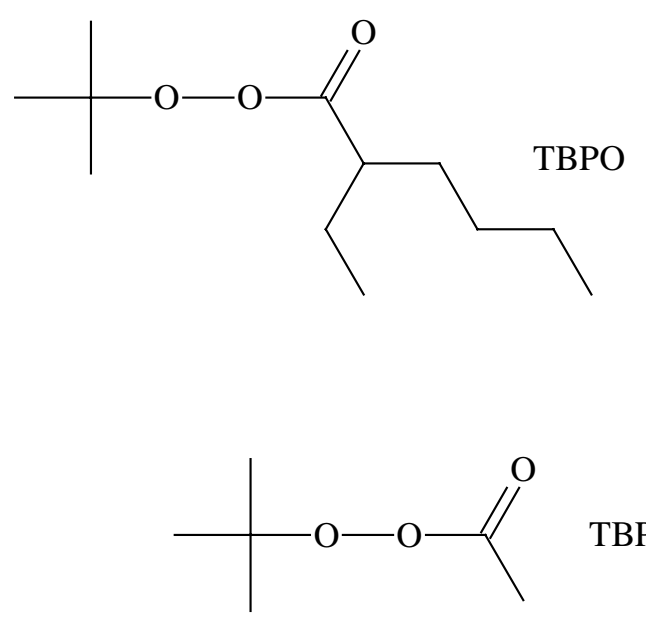

TBPA

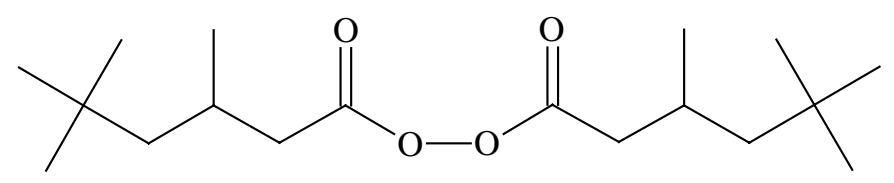

BTMHP

Abb. 4.8: Strukturformeln der untersuchten Peroxide. 


\subsubsection{Initiierungsexperiment mit einer BTMHP/TBPO Mischung}

In diesem Initiierungsexperiment wurde Bis-3,5,5-trimethylhexanoylperoxid mit einer Initiatorkonzentration von $c(\mathrm{BTMHP})_{0}=8.6 \cdot 10^{-6} \mathrm{~mol} \cdot \mathrm{L}^{-1}$ und tert-Butylperoxy-2ethylhexanoat mit $c(\mathrm{TBPO})_{0}=8.5 \cdot 10^{-6} \mathrm{~mol} \cdot \mathrm{L}^{-1}$ verwendet. Der Stoffmengenanteil an Propionaldehyd betrug 0.0032. Die Reaktionstemperatur lag zwischen 140 und $200{ }^{\circ} \mathrm{C}$. Der Ethenumsatz-Temperaturverlauf ist in Abbildung 4.9 gezeigt.

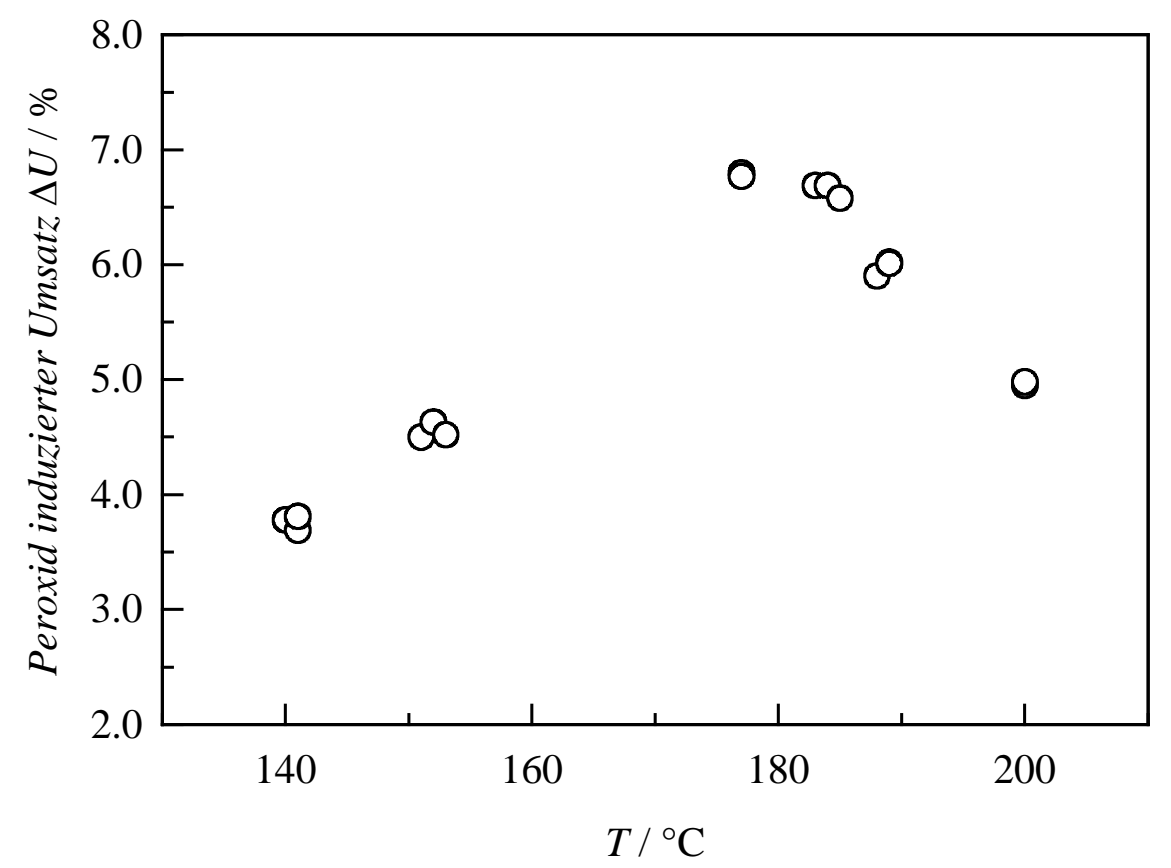

Abb.4.9: Temperaturabhängigkeit des Peroxid-induzierten Umsatzes für EthenHomopolymerisation bei 2000 bar mit einer Initiatormischung von TBPO und BTMHP; $p=2000 \mathrm{bar}, m_{E}=706 \mathrm{~g} \cdot \mathrm{h}^{-1} ; c(\mathrm{TBPO})_{0}=8.5 \cdot 10^{-6} \mathrm{~mol} \cdot \mathrm{L}^{-1}$; $c(\text { BTMHP })_{0}=8.6 \cdot 10^{-6} \mathrm{~mol} \cdot \mathrm{L}^{-1}$.

Bei einer Temperatur von 177 bis $184^{\circ} \mathrm{C}$ bildet sich ein Umsatzplateau. In diesem Bereich liegen die beiden optimalen Temperaturen der eingesetzten Initiatoren, so dass oberhalb der ersten optimalen Temperatur von BTMHP $\left(T_{\text {opt }}=177 \pm 5^{\circ} \mathrm{C}\right)$ die Abnahme des BTMHP induzierten Ethenumsatzes durch den Anstieg des TBPO induzierten Ethenumsatzes kompensiert wurde. Oberhalb der optimalen Temperatur von TBPO $\left(T_{\text {opt }}=188 \pm 5^{\circ} \mathrm{C}\right) \operatorname{sinkt}$ der gesamte Peroxid-induzierte Ethenumsatz ab.

Analog zur Herleitung der Formel zur Berechnung der Effektivität eines Peroxids (Gleichung 4.5 und 4.11) [1, 2, 3], lässt sich Gleichung 4.17 und 4.18 für Initiatormischungen ableiten. Gleichung 4.2 ergibt unter Berücksichtigung zweier Initiatoren: 


$$
\frac{\mathrm{d} c_{\mathrm{Ri}}}{\mathrm{d} t}=2 \cdot f_{1} \cdot k_{\mathrm{obs}, 1} \cdot c_{1, \mathrm{INI}}+2 \cdot f_{2} \cdot k_{\mathrm{obs}, 2} \cdot c_{2, \mathrm{INI}}-2 \cdot k_{\mathrm{t}} \cdot c_{\mathrm{Ri}}^{2}
$$

Nach dem Bodensteinprinzip lässt sich Gleichung (4.15) nach der Radikalkonzentration auflösen:

$$
c_{\mathrm{Ri}}=\sqrt{\frac{f_{1} \cdot k_{\mathrm{obs}, 1} \cdot c_{1, \mathrm{INI}}+f_{2} \cdot k_{\mathrm{obs}, 2} \cdot c_{2, \mathrm{INI}}}{k_{\mathrm{t}}}}
$$

Mit Gleichung 4.16 folgen Gleichung 4.17 und 4.18.

$$
\begin{aligned}
& f_{2}=\left[\left(\frac{\Delta U}{1-\Delta U}\right)^{2} \cdot \frac{k_{\mathrm{t}} \cdot \rho_{\mathrm{E}}}{k_{\mathrm{p}}{ }^{2} \cdot \tau^{2} \cdot \rho_{\mathrm{A}}}-f_{1} \cdot k_{\mathrm{obs}, 1} \cdot \frac{c_{1, \mathrm{INI}, 0}}{1+\tau \cdot k_{\mathrm{obs}, 1}}\right] \cdot \frac{1+\tau \cdot k_{\mathrm{obs}, 2}}{k_{\mathrm{obs}, 2} \cdot c_{2, \mathrm{INI}, 0}} \\
& f_{2}=\left[\exp (2 \Delta \mathrm{y})-f_{1}\right] \cdot \frac{C_{1, \mathrm{INI}, \text { zersetzt }}}{c_{2, \mathrm{INI}, \text { ersetzt }}}
\end{aligned}
$$

mit:

$c_{\mathrm{INI}, \mathrm{zersetzt}}=c_{\mathrm{INI}, 0}-c_{\mathrm{INI}}$

$c_{\mathrm{INI}}=\frac{c_{\mathrm{INI}, 0}}{1+\tau \cdot k_{\mathrm{obs}}}$, Initiatorkonzentration im CSTR

Wenn die Initiatoreffektivität eines Initiators $\left(f_{1}\right)$ bekannt ist, lässt sich aus den Umsatzdaten die Initiatoreffektivität des anderen Initiators $\left(f_{2}\right)$ berechnen, bzw. wenn beide bekannt sind, wird eine eventuelle Beeinflussung der Effektivität des einen Initiators durch den anderen erkennbar. In Abbildung 4.10 ist die mit Gleichung 4.16 berechnete Initiatoreffektivität von BTMHP gegen die Temperatur aufgetragen. 


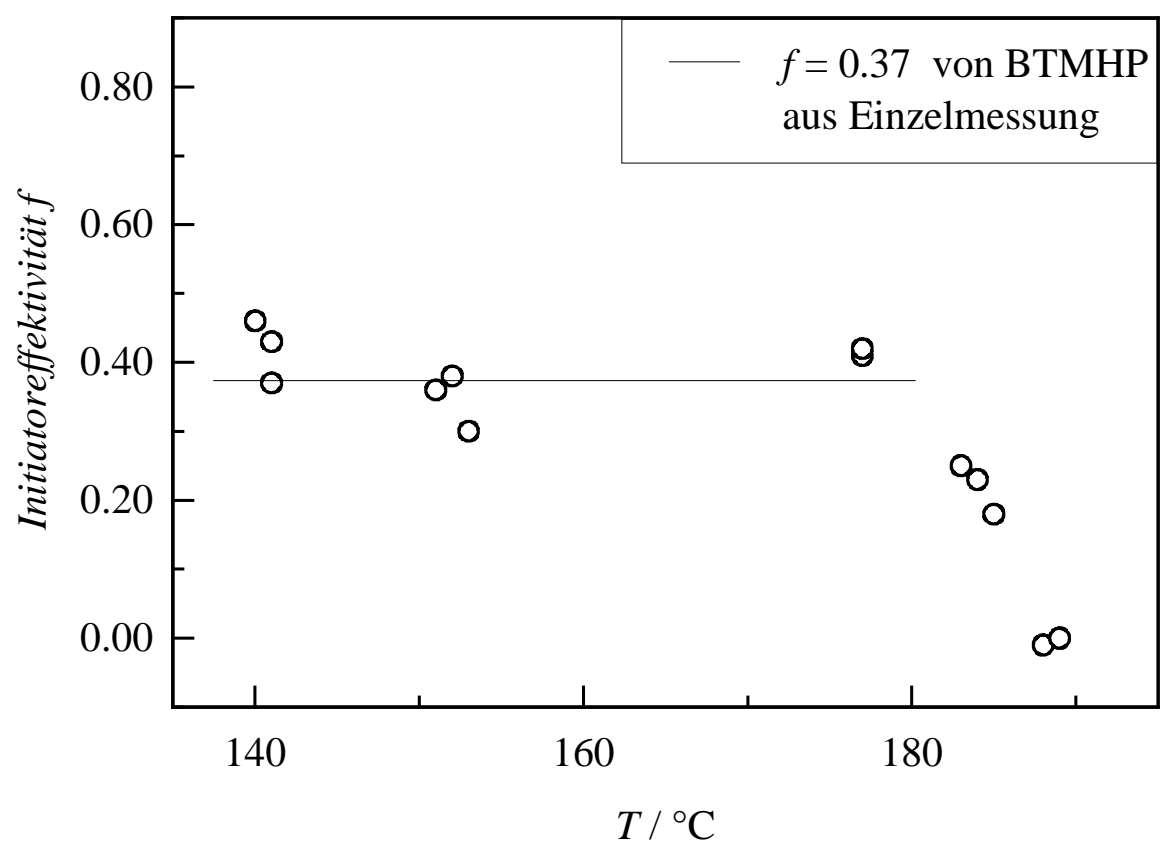

Abb. 4.10: Temperaturabhängigkeit der Initiatoreffektivität für BTMHP, berechnet mit aus der Literatur bekannten $f(T B P O)=0.62$ [2]. Die durchgezogene Linie gibt $f=0.37$ für BTMHP aus einer separaten Messung an [15]. $p=2000$ bar, $m_{E}=706 \mathrm{~g} \cdot \mathrm{h}^{-1} ; \quad c(\text { TBPO })_{0}=8.5 \cdot 10^{-6} \mathrm{~mol} \cdot \mathrm{L}^{-1}$; $c(\mathrm{BTMHP})_{0}=8.6 \cdot 10^{-6} \mathrm{~mol} \cdot \mathrm{L}^{-1}$.

Für TBPO ist die Effektivität von 0.62 aus der Literatur bekannt [2]. Die aus der Polymerisation mit dem Cocktail erhaltende Initiatoreffektivität für BTMHP ist 0.39 und stimmt mit dem vorher bestimmten Wert gut überein [15]. Die beiden Peroxide in der Mischung initiieren unabhängig die Ethen-Polymerisation und beeinflussen sich nicht gegenseitig.

\subsubsection{Initiierungsexperiment mit einer TBPP/TBPA Mischung}

In diesem Initiierungsexperiment wurde tert-Butylperoxypivalat mit einer Initiatorkonzentration von $c(\mathrm{TBPP})_{0}=9.8 \cdot 10^{-6} \mathrm{~mol} \cdot \mathrm{L}^{-1}$ und tert-Butylperoxyacetat mit $c(\mathrm{TBPA})_{0}=$ $5.0 \cdot 10^{-6} \mathrm{~mol} \cdot \mathrm{L}^{-1}$ verwendet. Der Stoffmengenanteil an Propionaldehyd betrug 0.0032. Die Reaktionstemperatur lag zwischen 160 und $217{ }^{\circ} \mathrm{C}$. Der Umsatz-Temperaturverlauf ist in Abbildung 4.11 gezeigt. Zum Vergleich sind in Abbildung 4.11 die in Messungen mit nur einem Initiator erhaltenen Peroxid-induzierten Umsätze von TBPP und TBPA $[3,16]$ dargestellt. Die jeweiligen Initiatorkonzentrationen des Cocktails entsprechen denen der Messungen mit nur einem Initiator. 


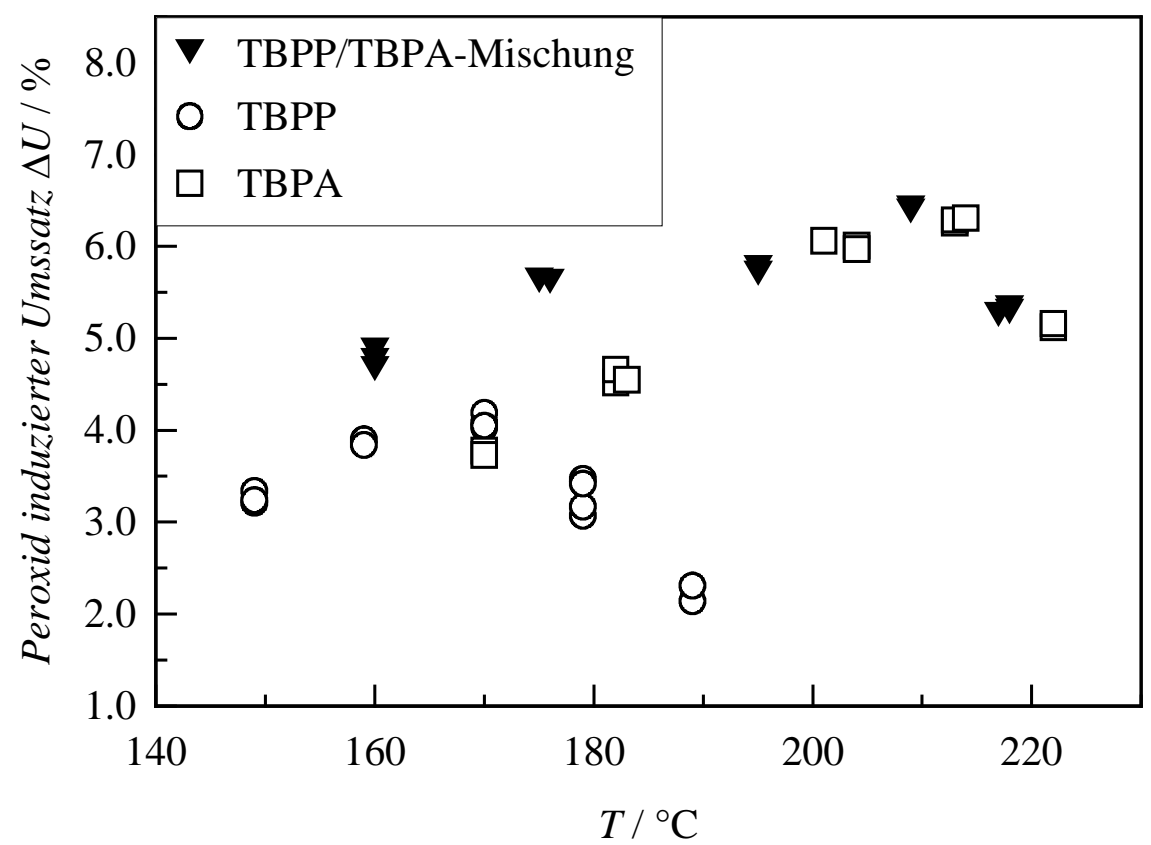

Abb. 4.11: Temperaturabhängigkeit der Peroxid-induzierten Umsätze für die Ethen-Homopolymerisation mit einer Initiatormischung von TBPP und TBPA (gefüllte Symbole) und die entsprechenden Messungen mit nur einem Initiator (offene Symbole) [3, 16]. Die verwendeten Initiatorkonzentrationen sind gleich. $p=2000$ bar, $m_{E}=706 \mathrm{~g} \cdot \mathrm{h}^{-1}, \quad c(\text { TBPP })_{0}=9.8 \cdot 10^{-6} \mathrm{~mol} \cdot \mathrm{L}^{-1}$, $c(\mathrm{TBPA})_{0}=5.0 \cdot 10^{-6} \mathrm{~mol} \cdot \mathrm{L}^{-1}$.

Der Peroxid-induzierte Umsatz der Mischung steigt bis $175{ }^{\circ} \mathrm{C}$ an und ist größer als der Peroxid-induzierte Umsatz der Einzelmessungen. Ab 175 bis $195{ }^{\circ} \mathrm{C}$ ist der Peroxidinduzierte Ethenumsatz der Mischung konstant oder durchläuft ein Minimum, da der von TBPA verursachte Umsatz mit der Temperatur ansteigt, aber der von TBPP induzierte Umsatz sinkt. Ab $195{ }^{\circ} \mathrm{C}$ steigt der Peroxid-induzierte Umsatz wieder bis zur optimalen Temperatur von TBPA $\left(T_{\text {opt }}=210 \pm 5^{\circ} \mathrm{C}\right)$ an und entspricht dem Umsatz aus der Einzelmessung mit TBPA. In diesem Temperaturbereich trägt TBPP nicht mehr zum Peroxidinduziertem Umsatz bei.

In Abbildung 4.12 ist für das Initiierungsexperiment der Mischung die mit Gleichung 4.16 bestimmten Initiatoreffektivität von TBPP dargestellt. Die zur Berechnung benutzte Initiatoreffektivität von TBPA ist 0.79 [16]. Die Effektivität der Einzelmessung mit TBPP ist $0.37[2]$. 


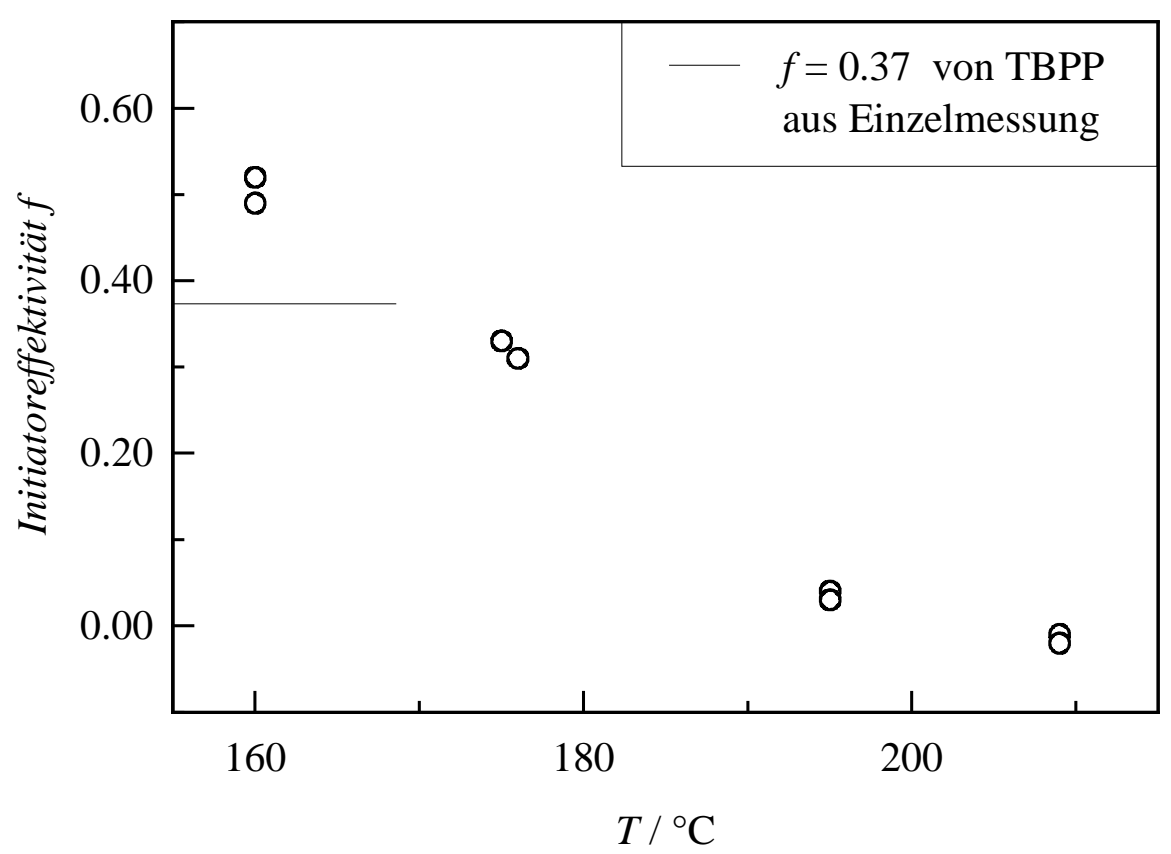

Abb. 4.12: Temperaturabhängigkeit der Initiatoreffektivität für TBPP berechnet mit f(TBPA) $=0.79$ [16]. Die durchgezogene Linie gibt $f=0.37$ für TBPP aus einer Einzelmessung an [2].

$p=2000$ bar, $\quad m_{E}=706 \mathrm{~g} \cdot \mathrm{h}^{-1}, \quad c(\text { TBPP })_{0}=9.8 \cdot 10^{-6} \mathrm{~mol} \cdot \mathrm{L}^{-1}$, $c(\text { TBPA })_{0}=5.0 \cdot 10^{-6} \mathrm{~mol} \cdot \mathrm{L}^{-1}$.

Wiederum ist bis $T_{\text {opt }}$ eine Übereinstimmung innerhalb der Messgenauigkeit der Initiatoreffektivitäten aus den Messungen mit nur einem Initiator mit den Effektivitäten, die im Initiatorcocktail erhalten worden sind, zu beobachten. Es gibt keine Anzeichen, dass die Radikale des bei tieferen Temperaturen zerfallenden Peroxyesters den langsamer zerfallenden Peroxyester beeinflussen.

\subsection{Asymmetrische Dialkylperoxide}

In der Arbeit von BECKER wurden bereits Initiatoreffektivitäten von symmetrischen Dialkylperoxiden untersucht [1, 2]. In der vorliegenden Arbeit wurden weitere Dialkylperoxide untersucht. Hierbei handelt es sich um asymmetrische Dialkylperoxide, wobei ein Alkylrest eine tert-Butylgruppe ist. Die in dieser Arbeit untersuchten Peroxide sind tert-Amyl-tert-butylperoxid (TATBP) und 1,1,2,2-Tetramethylpropyl-tert-butylperoxid (TMPTBP). Die Strukturformeln von TATBP und TMPTBP sind in Abbildung 4.13 gezeigt. 


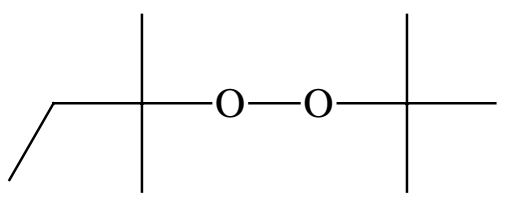

TATBP

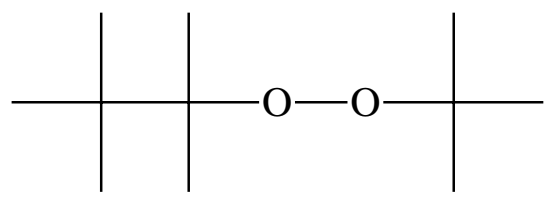

TMPTBP

Abb. 4.13: Strukturformeln der untersuchten Dialkylperoxide.

\subsubsection{Initiierungsexperiment mit tert-Amyl-tert-butylperoxid als Initiator}

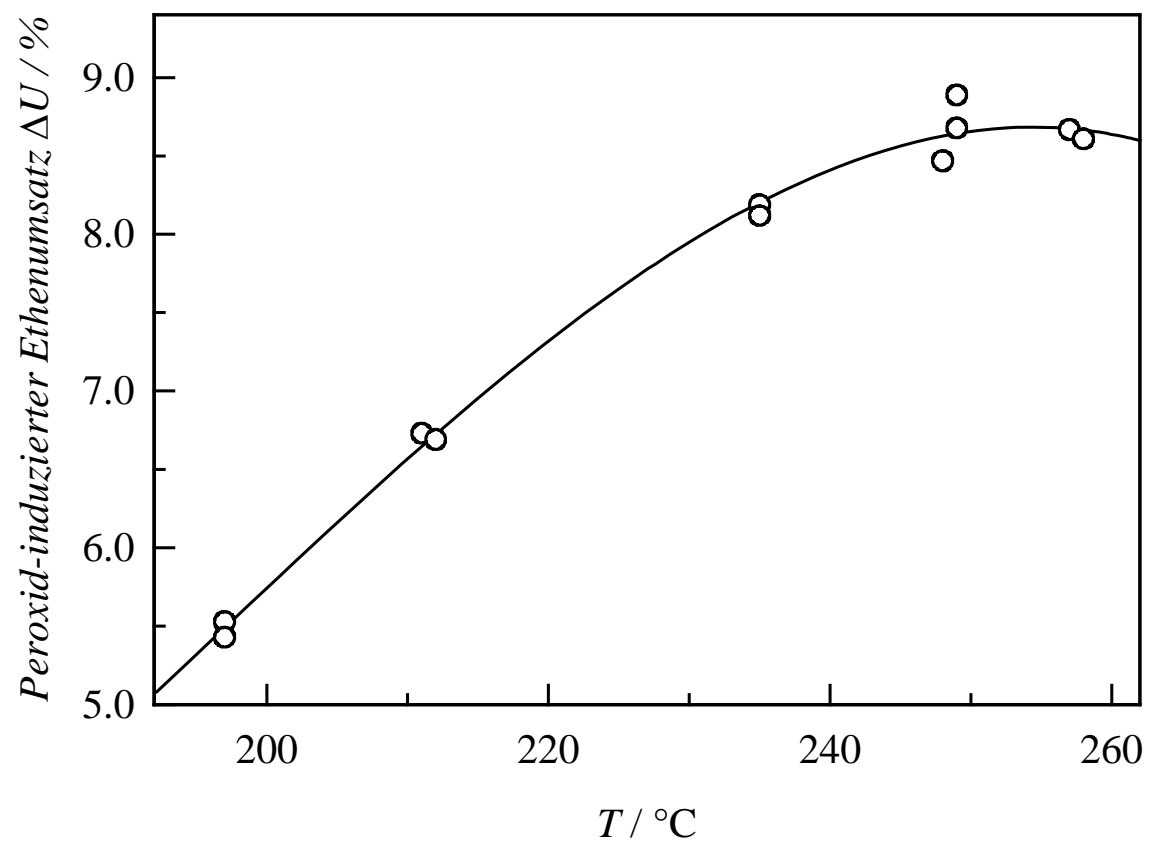

Abb. 4.14: Temperaturabhängigkeit des Peroxid-induzierten Ethenumsatzes für EthenHomopolymerisation mit TATBP als Initiator;

$p=2000 \mathrm{bar}, m_{E}=706 \mathrm{~g} \cdot \mathrm{h}^{-1} ; \mathrm{c}(\mathrm{TATBP})_{0}=3.9 \cdot 10^{-6} \mathrm{~mol} \cdot \mathrm{L}^{-1}$.

In diesem Initiierungsexperiment wurde TATBP mit einer Initiatorkonzentration von $c(\text { TATBP })_{0}=3.9 \cdot 10^{-6} \mathrm{~mol} \cdot \mathrm{L}^{-1}$ als Initiator verwendet. Der Stoffmengenanteil an Propionaldehyd betrug 0.0032. Die Reaktionstemperatur lag zwischen 197 bis $258{ }^{\circ} \mathrm{C}$. Die Temperaturabhängigkeit des Peroxid-induzierten Ethenumsatzes ist in Abbildung $4.14 \mathrm{zu}$ sehen. Die optimale Temperatur liegt bei $252 \pm 5{ }^{\circ} \mathrm{C}$.

Für diesen Initiator gibt es keine Hochdruckdaten für den thermischen Zerfall. In der Literatur gibt es eine Zerfallskinetik von MATSUYAMA, die in Cumol bei 1 bar gemessen worden ist [17].

Da die symmetrischen Peroxide Di-tert-Butylperoxid (DTBP) und Di-tert-Amylperoxid (DTAP) ein Aktivierungsvolumen von $\Delta V^{\ddagger}=10.1 \mathrm{~cm}^{3} \cdot \mathrm{mol}^{-1}$ besitzen, kann dies in guter 
Näherung auch für TATBP angenommen werden. Die Temperaturabhängigkeit der Initiatoreffektivität, die mit dieser Kinetik erhalten worden ist, ist in Abbildung 4.15 gezeigt. Es wird eine Effektivität erhalten, die schon vor der optimalen Temperatur $\left(T_{\text {opt }}=252{ }^{\circ} \mathrm{C}\right)$ absinkt.

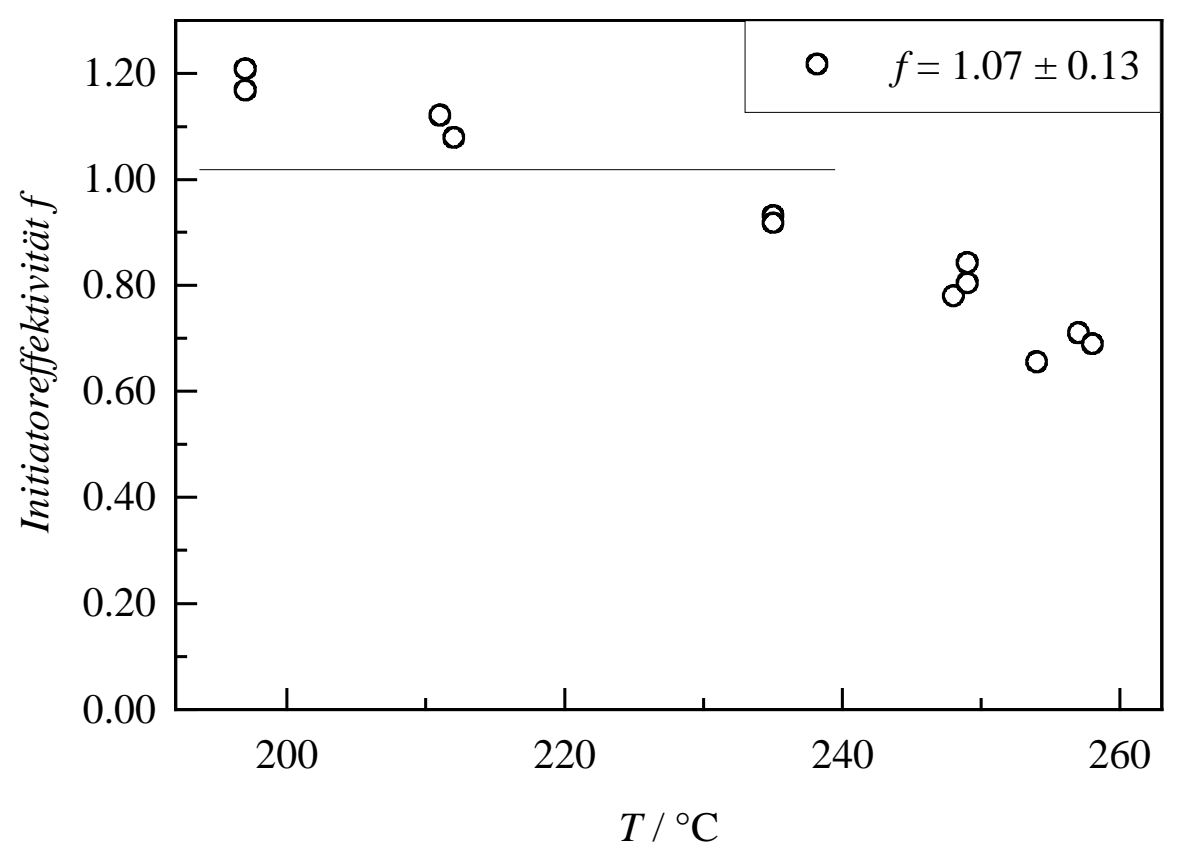

Abb. 4.15: Temperaturabhängigkeit der Initiatoreffektivität für TATBP. Verwendete Zerfallskinetik von MATSUYAMA [17]. Die durchgezogene Linie stellt den Mittelwert dar. $p=2000 \mathrm{bar}, m_{E}=706 \mathrm{~g} \cdot \mathrm{h}^{-1} ; \mathrm{c}(\mathrm{TATBP})_{0}=3.9 \cdot 10^{-6} \mathrm{~mol} \cdot \mathrm{L}^{-1}$.

Ein Grund für das Absinken könnte ein Fehler in $k_{\text {obs }}$ sein, der durch die Extrapolation der von Matsuyama bei $120{ }^{\circ} \mathrm{C}$ bis $140{ }^{\circ} \mathrm{C}$ gemessenen Kinetik erhalten wurde [17]. Deswegen wurde versucht, mit einer Zerfallskinetik, die aus der Zerfallskinetik von DTBP und DTAP konstruiert worden ist, eine bessere Temperaturabhängigkeit zu erhalten. Dazu wurden die Aktivierungsparameter von DTBP und DTAP aus der Literatur genommen und gemittelt [18, 19]. In Tabelle 4.1 sind die Aktivierungsparameter aufgelistet. Die Aktivierungsenergie der Hochdruckdaten wurde mit folgender Gleichung auf verschwindenden Druck berechnet:

$$
E_{\mathrm{A}}(0 \text { bar })=E_{\mathrm{A}}(p)-p \cdot \Delta V^{\mp} ; p \text { ist der Druck, bei dem } E_{\mathrm{A}} \text { bestimmt worden ist. }
$$

Somit sind die Hochdruckdaten mit der Kinetik von MATSUYAMA vergleichbar. 


\begin{tabular}{cccc}
\hline Peroxid & $\boldsymbol{E}_{\mathbf{A}}(\mathbf{0} \mathbf{~ b a r}) / \mathbf{~} \mathbf{J} \cdot \mathbf{m o l}^{\mathbf{- 1}}$ & $\boldsymbol{A} / \mathbf{s}^{\mathbf{- 1}}$ & $\Delta \boldsymbol{V}^{\neq} / \mathbf{~ c m}^{\mathbf{3}} \cdot \mathbf{m o l}^{\mathbf{- 1}}$ \\
\hline DTBP [18] & 150.9 & $1.20 \cdot 10^{15}$ & 10.1 \\
DTAP [19] & 150.9 & $2.76 \cdot 10^{15}$ & 10.1 \\
TATBP (abgeschätzt) & 150.9 & $1.98 \cdot 10^{15}$ & 10.1 \\
TATBP [17] & 157.3 & $1.09 \cdot 10^{16}$ & - \\
\hline
\end{tabular}

Tab. 4.1: Präexponentieller Faktor und Aktivierungsparameter von DTBP und DTAP [18, 19], sowie die durch Mittelung abgeschätzten Werte für TATBP. Zum Vergleich die Zerfallskinetik von MATSUYAMA [17]

In Abbildung 4.16 ist die Temperaturabhängigkeit der mit der konstruierten Zerfallskinetik (siehe Tabelle 4.1) berechneten Initiatoreffektivität gezeigt.

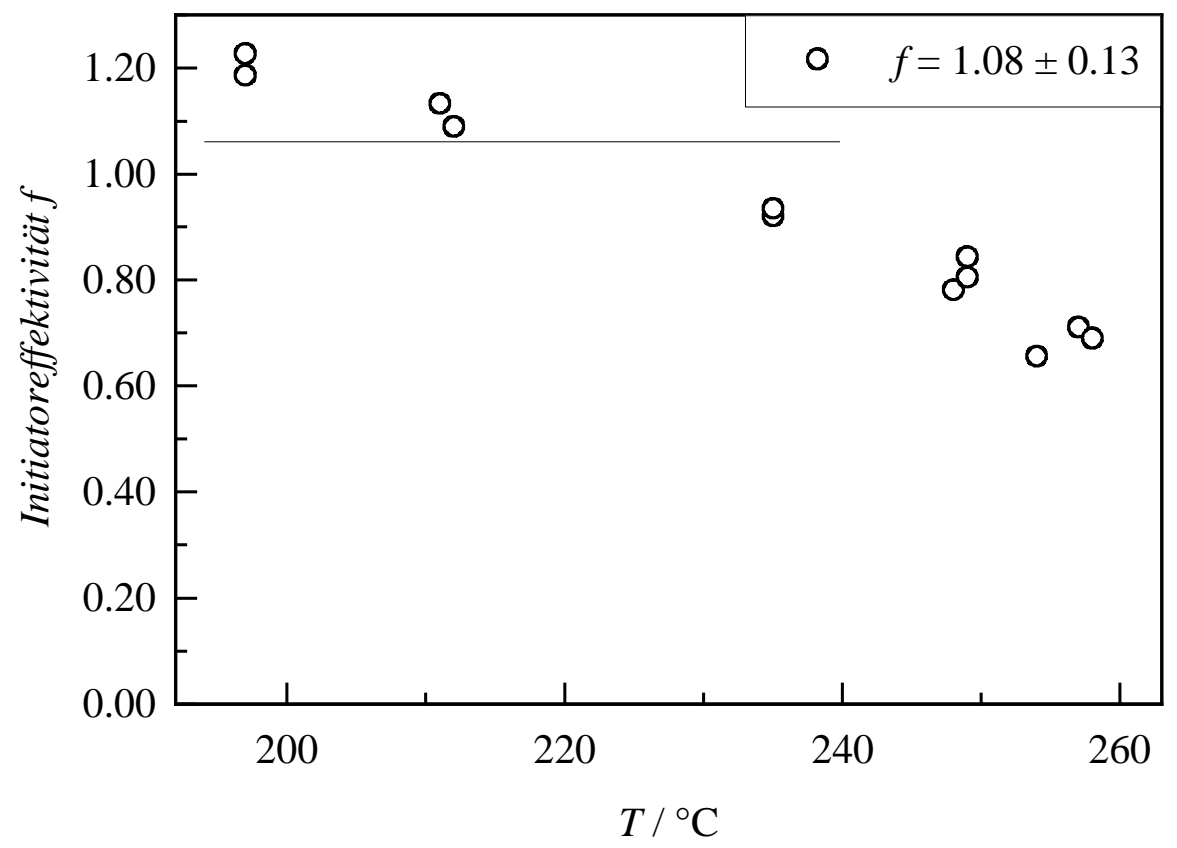

Abb. 4.16: Temperaturabhängigkeit der Initiatoreffektivität für TATBP. Berechnet mit abgeschätzter Zerfallskinetik, siehe Tabelle 4.1. Die durchgezogene Linie stellt den Mittelwert von $f$ dar. $p=2000 \mathrm{bar}, m_{E}=706 \mathrm{~g} \cdot \mathrm{h}^{-1} ; \mathrm{c}(\mathrm{TATBP})_{0}=3.9 \cdot 10^{-6} \mathrm{~mol} \cdot \mathrm{L}^{-1}$.

Die erhaltenden Effektivitäten sind nahezu identisch mit den, mit der Zerfallskinetik von Matsuyama berechneten, $f$-Werten. Es scheint, dass die optimale Temperatur vor $252{ }^{\circ} \mathrm{C}$ bereits durchlaufen ist, obwohl bei dieser Temperatur der Ethenumsatz angestiegen ist. Deshalb sind in Abbildung 4.15 und 4.16 nur die Effektivitäten der ersten drei Temperaturen zur Berechnung des Mittelwertes von $f$ herangezogen worden. Die mit der Zerfallskinetik von MATSUYAMA berechnete Initiatoreffektivität ist 1.07. $f$ mit der abgeschätzten Zerfallskinetik berechnet ist nahezu identisch und liegt bei 1.08. Die optimale Temperatur sollte zwischen 
235 und $249^{\circ} \mathrm{C}$ liegen. Frühere Arbeiten haben gezeigt, dass die optimale Temperatur mit der Zerfallskinetik korreliert. Bei $T_{\mathrm{opt}}$ ist $k_{\mathrm{obs}} \approx 0.5 \mathrm{~s}^{-1}$. Mit der MATSUYAMA Zerfallskinetik wird eine optimale Temperatur von $236.5{ }^{\circ} \mathrm{C}$ und mit der konstruierten Kinetik eine optimale Temperatur von $239{ }^{\circ} \mathrm{C}$ vorausgesagt. Ein weiterer Beleg dafür, dass $T_{\text {opt }}$ unter $249{ }^{\circ} \mathrm{C}$ liegt. JAUER hat ebenfalls durch Mittelung der Zerfallskinetiken zweier verschiedener Initiatoren eine Kinetik für einen dritten Initiator abgeschätzt und damit zufriedenstellende Ergebnisse erhalten [11].

\subsubsection{Initiierungsexperiment mit 1,1,2,2-Tetramethylpropyl-tert-butylperoxid als Initiator}

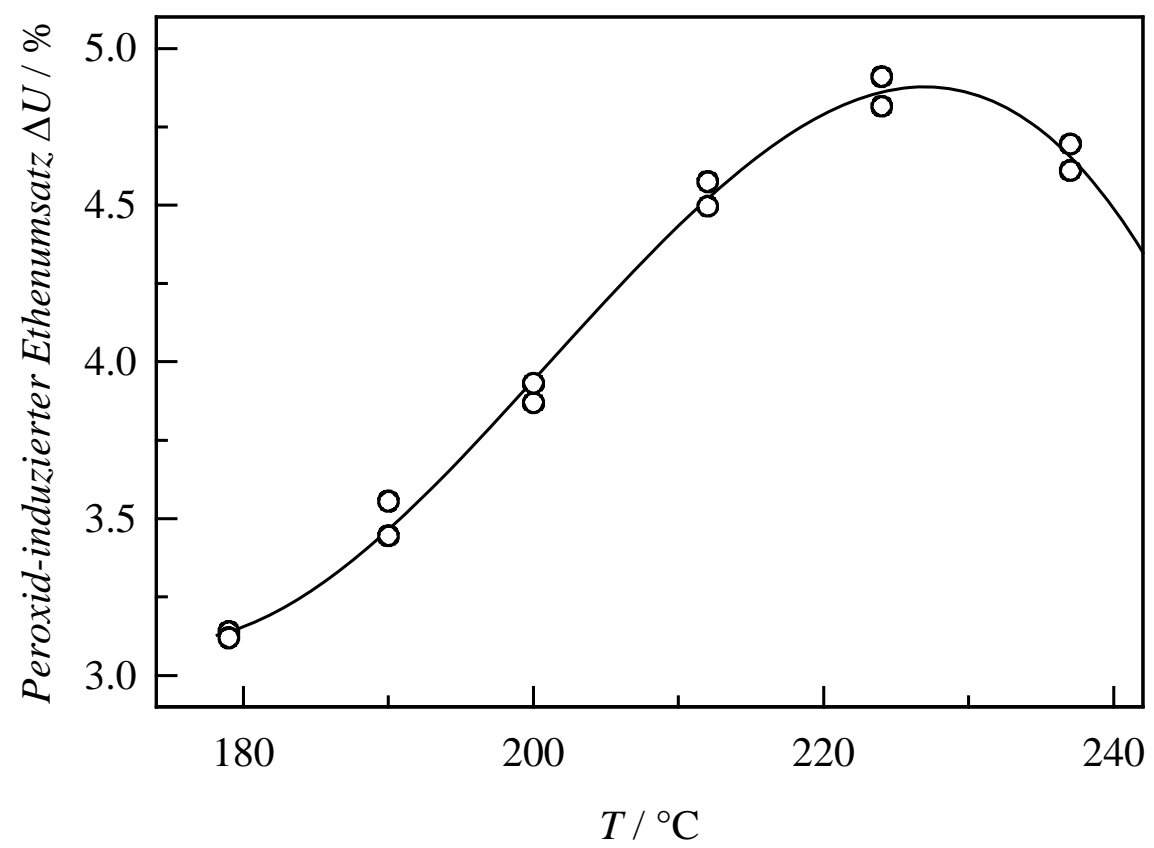

Abb. 4.17: Temperaturabhängigkeit des Peroxid-induzierten Ethenumsatzes für EthenHomopolymerisation mit TMPTBP als Initiator; $p=2000 \mathrm{bar}, m_{E}=706 \mathrm{~g} \cdot \mathrm{h}^{-1} ; \mathrm{c}(\mathrm{TMPTBP})_{0}=2.6 \cdot 10^{-6} \mathrm{~mol} \cdot \mathrm{L}^{-1}$;

In Abbildung 4.17 ist der mit TMPTBP induzierte Ethenumsatz zu sehen. Die Reaktionstemperaturen lagen zwischen 179 und $237{ }^{\circ} \mathrm{C}$. Die Initiatorkonzentration betrug $\mathrm{c}(\mathrm{TMPTBP})_{0}=2.6 \cdot 10^{-6} \mathrm{~mol} \cdot \mathrm{L}^{-1}$. Der Stoffmengenanteil an Propionaldehyd betrug 0.0032 . $T_{\text {opt }}$ lag bei $225 \pm 5^{\circ} \mathrm{C}$.

In Abbildung 4.18 ist die Temperaturabhängigkeit der Initiatoreffektivität von TMPTBP gezeigt. Die zur Berechnung der Effektivität benutzte Zerfallskinetik wurde von VöGELE in $n$-Heptan in einem weiten Zustandsbereich bestimmt [19]. $f$ bei $179{ }^{\circ} \mathrm{C}$ ist leicht erhöht im Vergleich zu den anderen Temperaturen. Die Punkte in der Abbildung 4.18 streuen, doch 
durch die hohe Anzahl der Datenpunkten ist eine befriedigend genaue Bestimmung der Initiatoreffektivität möglich. Es wird eine Effektivität von 0.72 erhalten.

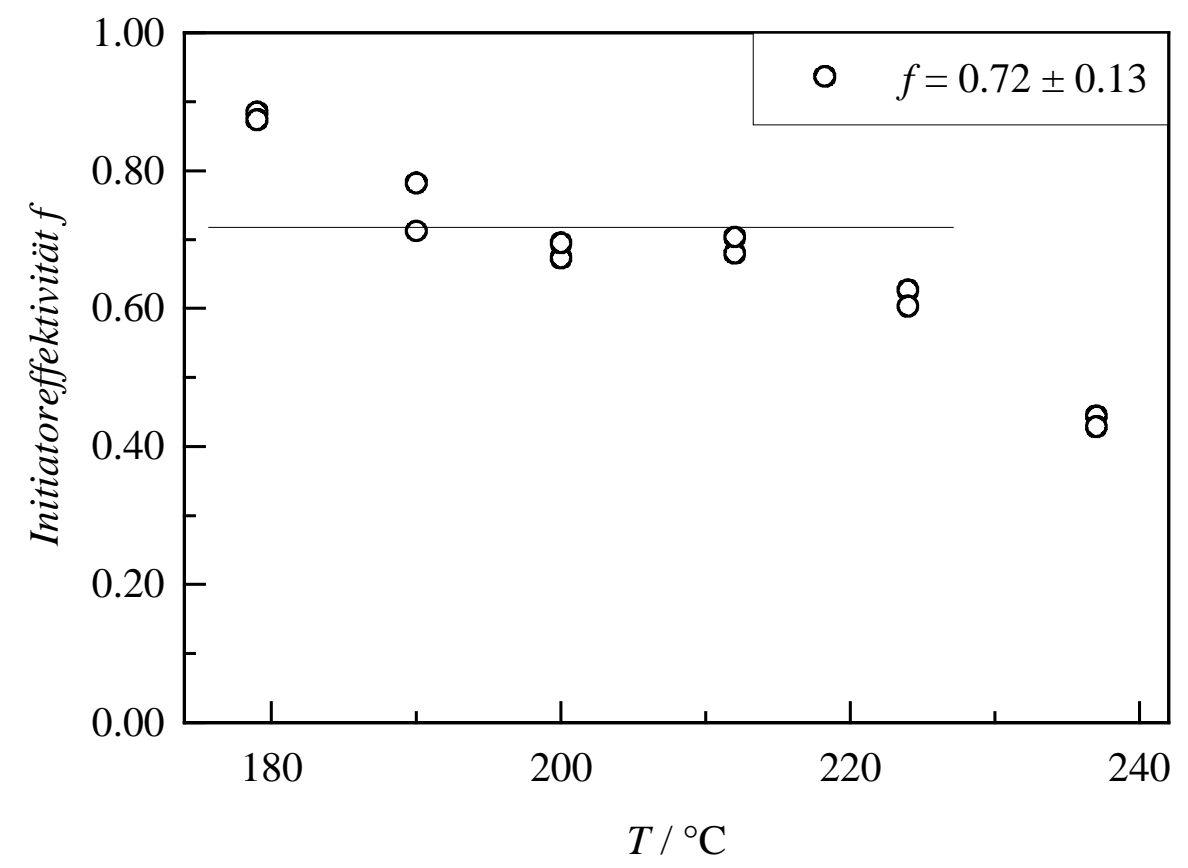

Abb. 4.18: Temperaturabhängigkeit der Initiatoreffektivität für TMPTBP. $p=2000 \mathrm{bar}, m_{E}=706 \mathrm{~g} \cdot \mathrm{h}^{-1} ; \mathrm{c}(\mathrm{TMPTBP})_{0}=2.6 \cdot 10^{-6} \mathrm{~mol} \cdot \mathrm{L}^{-1}$;

\section{7 tert-Amylperoxyester}

Wie bei den asymmetrischen Dialkylperoxiden soll der Einfluss der strukturellen Unterschiede auf die Initiatoreffektivität untersucht werden. Peroxyester bestehen aus einer Alkohol- und einer Säureseite. In Abbildung 4.19 wird dies verdeutlich. Die Namen Alkoholund Säureseite stammen von den Edukten (Alkohol und Säure) der Estersynthese und werden ebenfalls bei Peroxyestern verwendet. Beide Seiten beeinflussen die Zerfallskinetik und Initiatoreffektivität unterschiedlich. Auch wird ersichtlich, welchen Einfluss die Reste R und R' haben, denn je nach Stabilität der Radikale R· bzw. R'· erfolgt die Folgereaktion nach dem primären Bindungsbruch unterschiedlich schnell. Je stabiler das Radikal ist, das bei der $\beta$ scission bzw. Decarboxylierung entsteht, desto schneller erfolgt die $\beta$-scission bzw. Decarboxylierung. Auch der primäre Bindungsbruch erfolgt schneller, wenn stabilere Radikale bei den Folgereaktionen entstehen [20, 13]. 


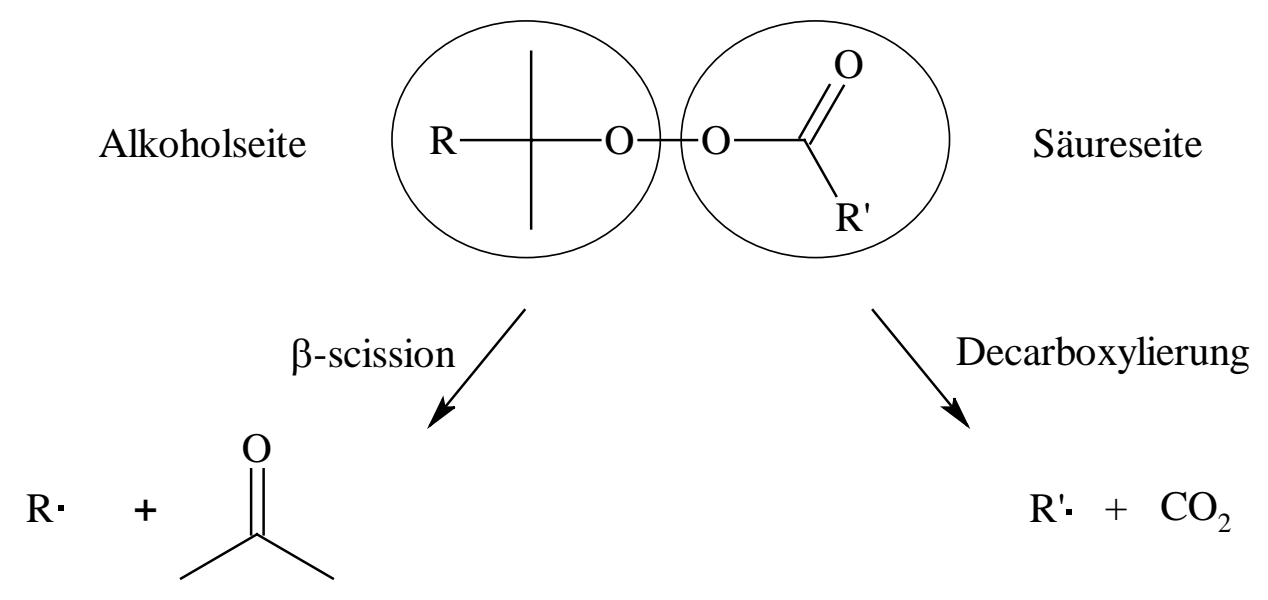

Abb. 4.19: Allgemeine Strukturformel von Peroxyestern mit Alkohol- und Säureseite, sowie die Folgereaktionen der Radikale nach dem primären Bindungsbruch.

Zunächst soll der Einfluss der Säureseite R' untersucht werden. Die Säureseite kann in drei Klassen unterteilt werden. Wenn das $\alpha$-C-Atom zur Carboxylgruppe ein primäres ist, wird der Peroxyester primär genannt. Dies bedeutet, dass nach dem Zerfall des Peroxyesters bei der anschließenden Decarboxylierung ein primäres Radikal R' entsteht. Ist das $\alpha$-C-Atom zur Carboxylgruppe sekundär bzw. tertiär, dann handelt es sich um sekundäre bzw. tertiäre Peroxyester.

In diesem Abschnitt werden die Initiatoreffektivitäten von fünf tert-Amylperoxyestern vorgestellt. Dabei handelt es sich um tert-Amylperoxyacetetat (TAPA), tert-Amylperoxy- $n$ butyrat (TAPnB), tert-Amylperoxy-iso-butyrat (TAPiB), tert-Amylperoxy-2-ethylhexanoat (TAPO) und tert-Amylperoxypivalat (TAPP). Die ersten beiden tert-Amylperoxyester, TAPA und TBPnB, sind primäre Peroxyester bezüglich des $\alpha$-Kohlenstoffatoms der Säureseite. TAPiB und TAPO sind sekundäre, TAPP ein tertiärer Peroxyester. Die Strukturformeln der untersuchten tert-Amylperoxyester sind in Abbildung 4.20 zu sehen. 
<smiles>CCCC(=O)OOC(C)(C)CC</smiles><smiles>CCCCC(CC)C(=O)OOC(C)(C)CC</smiles><smiles>CCC(C)(C)OOC(=O)C(C)(C)[AlH2]</smiles>

Abb. 4.20: Strukturformeln der untersuchten tert-Amylperoxyester.

\begin{tabular}{cccc}
\hline Peroxid & $\boldsymbol{E}_{\mathbf{A}}(\mathbf{0} \mathbf{b a r}) / \mathbf{k J} \cdot \mathbf{m o l}^{\mathbf{- 1}}$ & $\mathbf{A} / \mathbf{s}^{\mathbf{- 1}}$ & $\Delta \boldsymbol{V}^{\neq} / \mathbf{c m}^{\mathbf{3}} \cdot \mathbf{m o l}^{-\mathbf{1}}$ \\
\hline TAPA & 141.2 & $1.52 \cdot 10^{15}$ & 12.5 \\
TAPnB & 139.6 & $1.03 \cdot 10^{15}$ & 8.2 \\
TAPiB & 132.9 & $7.53 \cdot 10^{14}$ & 2.6 \\
TAPO & 130.5 & $7.27 \cdot 10^{14}$ & 2.8 \\
TAPP & 122.7 & $4.11 \cdot 10^{14}$ & 2.4 \\
\hline
\end{tabular}

Tab.4.2: Präexponentieller Faktor und Aktivierungsparameter der Geschwindigkeitskonstante des thermischen Zerfalls für die untersuchten tert-Amylperoxyester [20].

Die zur Bestimmung der Initiatoreffektivität benutzten Zerfallskoeffizienten wurden aus der Literatur entnommen [20]. Die Aktivierungsparameter des Geschwindigkeitskoeffizienten des thermischen Zerfalls sind in Tabelle 4.2 aufgelistet. 


\subsubsection{Initiierungsexperiment mit TAPA als Initiator}

In Abbildung 4.21 ist der Peroxid-induzierte Ethenumsatz von TAPA gegen die Temperatur aufgetragen. Die Initiatorkonzentration betrug $c(\text { TAPA })_{0}=4 \cdot 4 \cdot 10^{-6} \mathrm{~mol} \cdot \mathrm{L}^{-1}$. Der Stoffmengenanteil des Propionaldehyds betrug 0.0032. Die Reaktionstemperatur lag zwischen 165 und $208^{\circ} \mathrm{C}$. Die optimale Temperatur $T_{\text {opt }}$ betrug $200 \pm 5^{\circ} \mathrm{C}$.

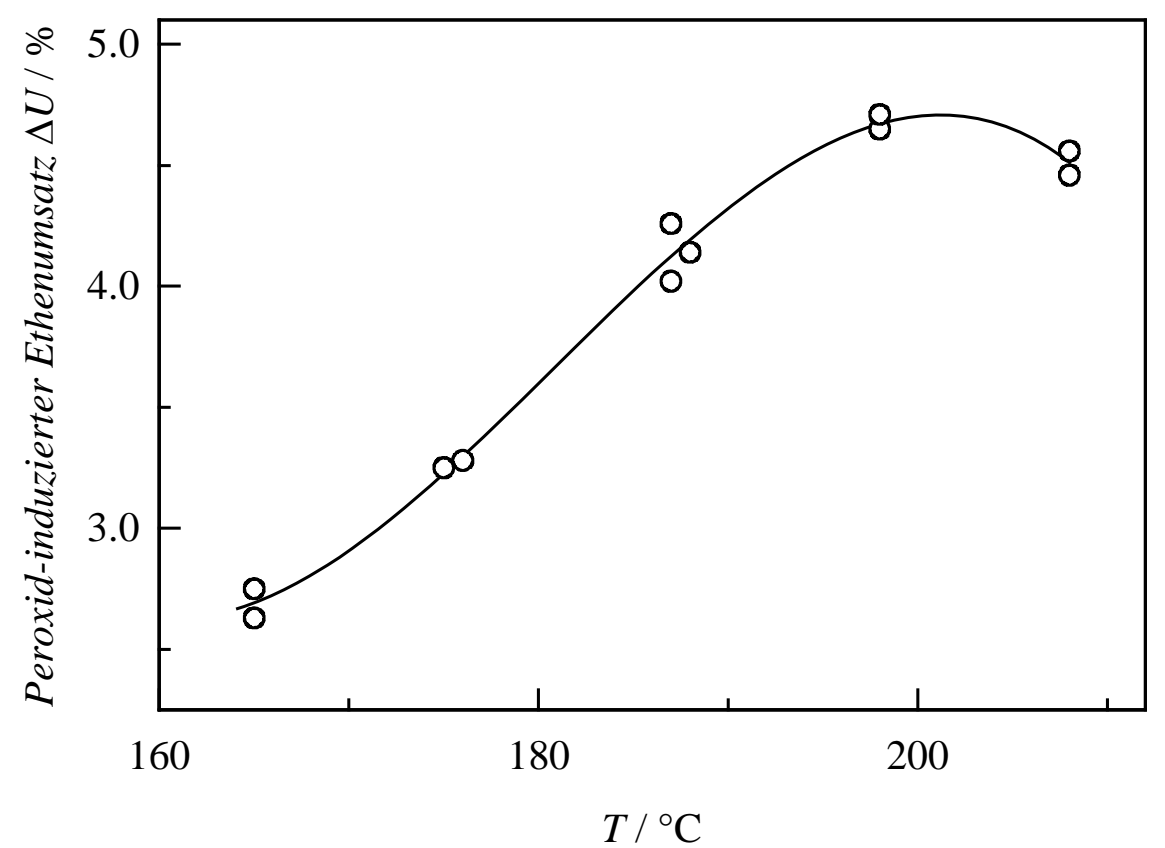

Abb. 4.21: Temperaturabhängigkeit des Peroxid-induzierten Ethenumsatzes für EthenHomopolymerisation mit TAPA als Initiator;

$p=2000 \mathrm{bar}, m_{E}=706 \mathrm{~g} \cdot \mathrm{h}^{-1} ; \mathrm{c}(\mathrm{TAPA})_{0}=4.4 \cdot 10^{-6} \mathrm{~mol} \cdot \mathrm{L}^{-1}$.

In Abbildung 4.22 ist die Temperaturabhängigkeit der Initiatoreffektivität von TAPA gezeigt. Die durchgezogene Linie stellt den Mittelwert der Initiatoreffektivität dar. Es wird eine Effektivität von 0.69 erhalten. 


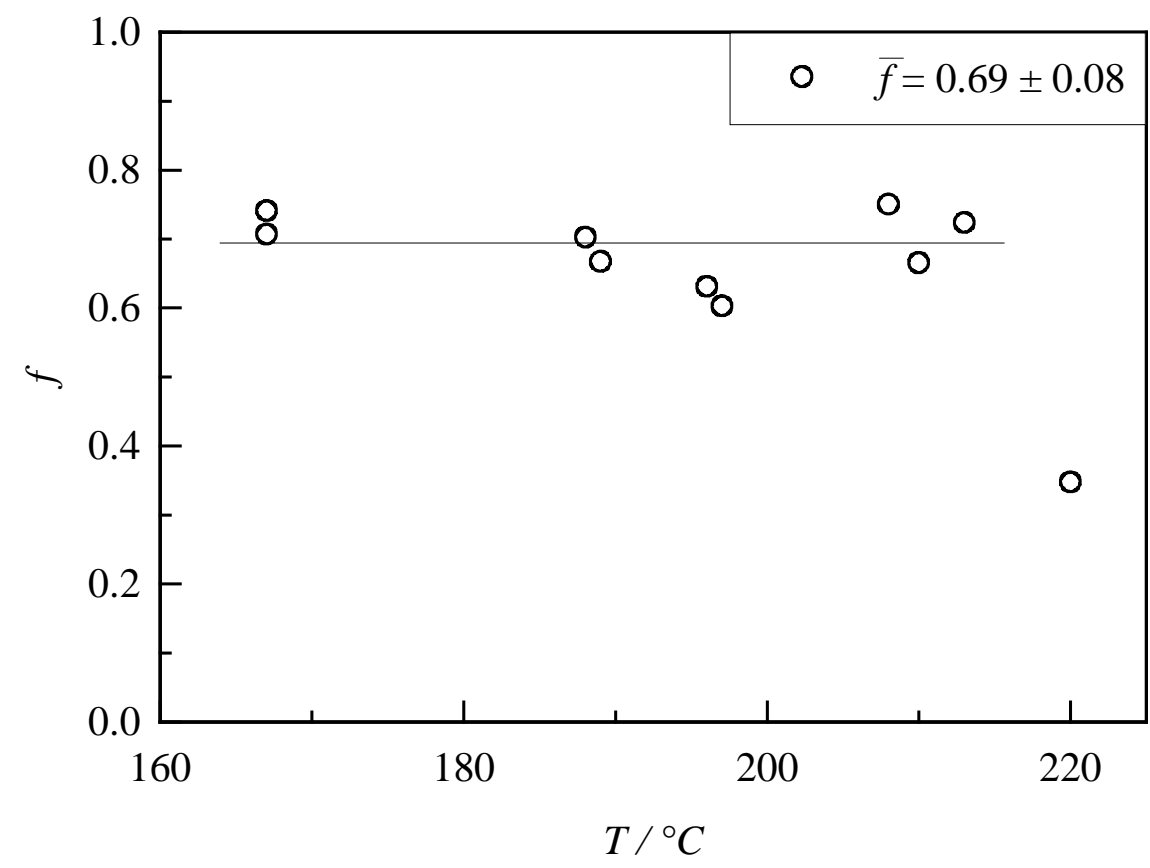

Abb. 4.22: Temperaturabhängigkeit der Initiatoreffektivität für TAPA.

$p=2000 \mathrm{bar}, m_{E}=706 \mathrm{~g} \cdot \mathrm{h}^{-1} ; \mathrm{c}(\mathrm{TAPA})_{0}=4.4 \cdot 10^{-6} \mathrm{~mol} \cdot \mathrm{L}^{-1}$.

\subsubsection{Initiierungsexperiment mit TAPnB als Initiator}

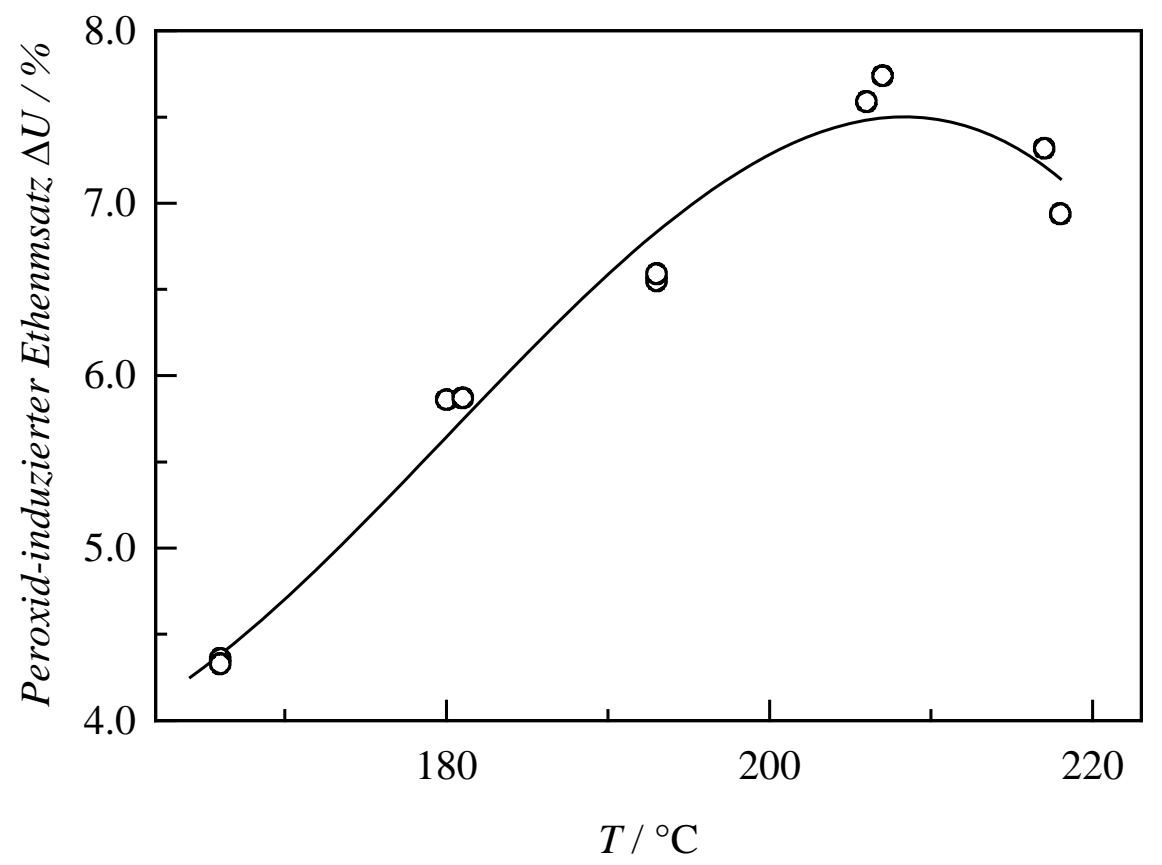

Abb. 4.23: Temperaturabhängigkeit des Peroxid-induzierten Ethenumsatzes für EthenHomopolymerisation mit TAPnB als Initiator;

$p=2000 \mathrm{bar}, m_{E}=706 \mathrm{~g} \cdot \mathrm{h}^{-1} ; \mathrm{c}(\mathrm{TAPnB})_{0}=8.6 \cdot 10^{-6} \mathrm{~mol} \cdot \mathrm{L}^{-1}$.

Die Initiatorkonzentration von TAPnB betrug $c(\mathrm{TAPnB})_{0}=8.6 \cdot 10^{-6} \mathrm{~mol} \cdot \mathrm{L}^{-1}$. Der Stoffmengenanteil an Propionaldehyd betrug 0.0032. Die Reaktionstemperatur lag zwischen 
166 und $217^{\circ} \mathrm{C} . T_{\text {opt }}$ betrug $209 \pm 5^{\circ} \mathrm{C}$. Der Umsatz-Temperaturverlauf ist in Abbildung 4.23 zu sehen.

Die aus den Ethenumsätzen bestimmten Effektivitäten sind in Abhängigkeit der Temperatur in Abbildung 4.24 zu sehen. Der Mittelwert der Initiatoreffektivität ist 0.73.

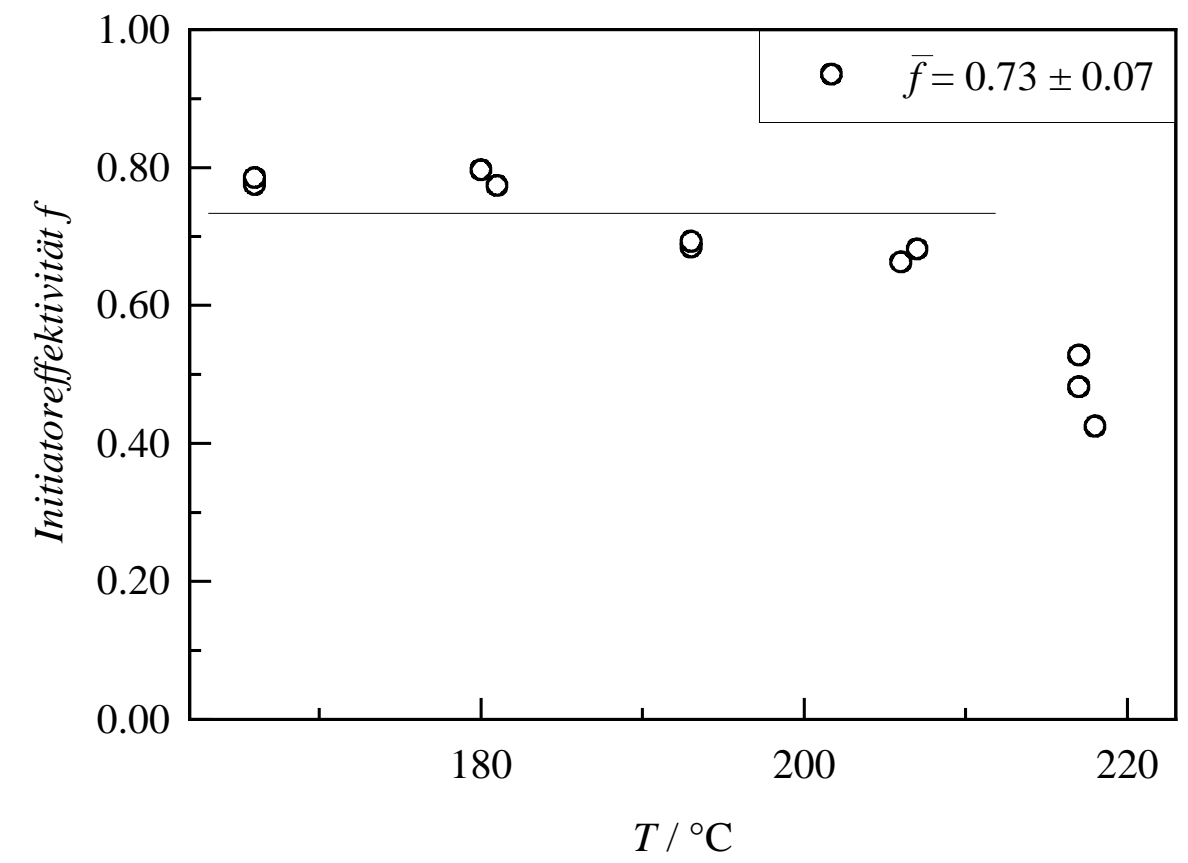

Abb. 4.24: Temperaturabhängigkeit der Initiatoreffektivität für TAPnB. $p=2000 \mathrm{bar}, m_{E}=706 \mathrm{~g} \cdot \mathrm{h}^{-1} ; \mathrm{c}(\mathrm{TAPnB})_{0}=8.6 \cdot 10^{-6} \mathrm{~mol} \cdot \mathrm{L}^{-1}$.

\subsubsection{Initiierungsexperiment mit TAPiB als Initiator}

In diesem Experiment betrug die Initiatorkonzentration von TAPiB $c(\mathrm{TAPiB})_{0}=$ $7.4 \cdot 10^{-6} \mathrm{~mol} \cdot \mathrm{L}^{-1}$. Die Reaktionstemperatur lag zwischen 138 und $195{ }^{\circ} \mathrm{C}$. Die optimale Temperatur wurde zu $184 \pm 5^{\circ} \mathrm{C}$ bestimmt. In Abbildung 4.25 ist der Peroxid-induzierte Ethenumsatz gegen die Reaktionstemperatur aufgetragen. Der Ethenumsatz steigt mit der Temperatur von $2.1 \%$ bei $138^{\circ} \mathrm{C}$ bis auf $4.7 \%$ bei $184^{\circ} \mathrm{C}$. 


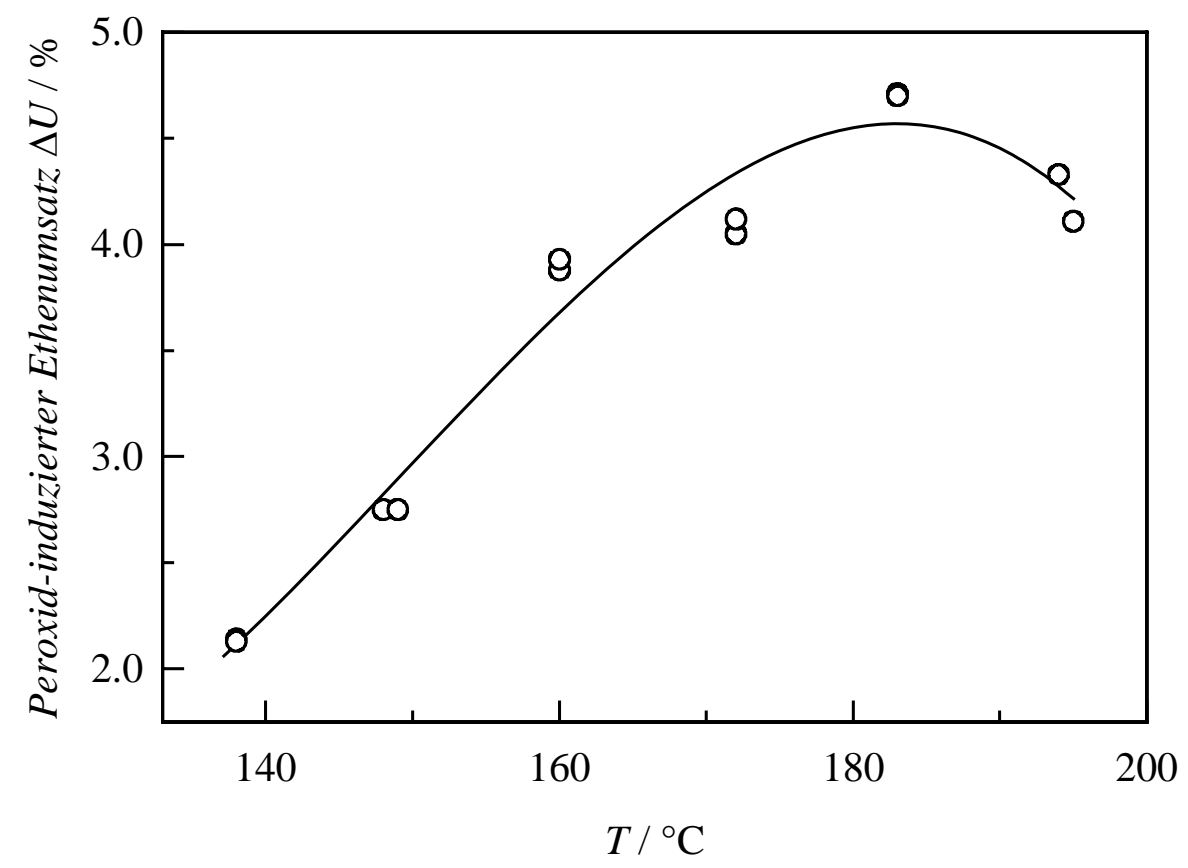

Abb. 4.25: Temperaturabhängigkeit des Peroxid-induzierten Ethenumsatzes für EthenHomopolymerisation mit TAPiB als Initiator; $p=2000 \mathrm{bar}, m_{E}=706 \mathrm{~g} \cdot \mathrm{h}^{-1} ; \mathrm{c}(\mathrm{TAPiB})_{0}=7.4 \cdot 10^{-6} \mathrm{~mol} \cdot \mathrm{L}^{-1}$.

Die erhaltene Temperaturabhängigkeit der Initiatoreffektivität ist in Abbildung 4.26 dargestellt.

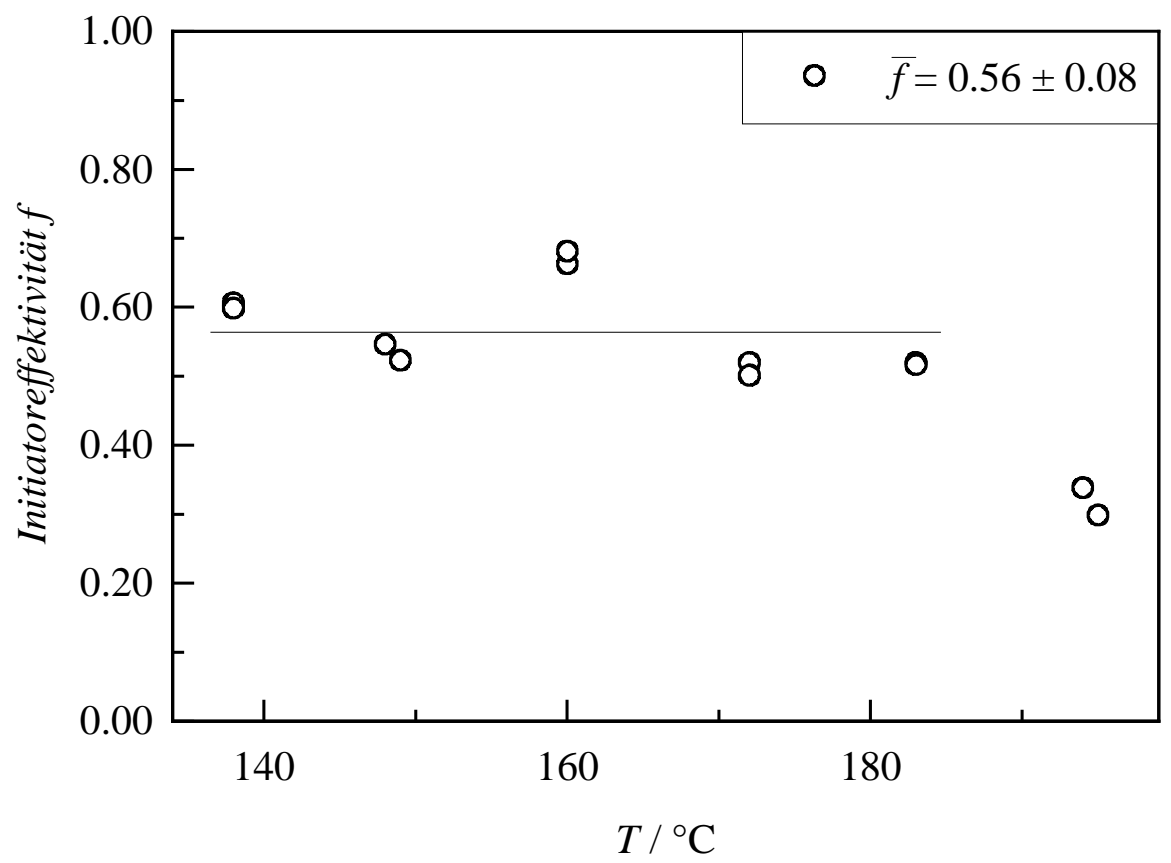

Abb. 4.26: Temperaturabhängigkeit der Initiatoreffektivität für TAPiB. $p=2000 \mathrm{bar}, m_{E}=706 \mathrm{~g} \cdot \mathrm{h}^{-1} ; \mathrm{c}(\mathrm{TAPiB})_{0}=7.4 \cdot 10^{-6} \mathrm{~mol} \cdot \mathrm{L}^{-1}$. 
Bei dieser Messung ist eine starke Streuung der Initiatoreffektivitäten zu sehen. Der Mittelwert von $f$ ist 0.56 .

\subsubsection{Initiierungsexperiment mit TAPO als Initiator}

In diesem Experiment betrug die Anfangsinitiatorkonzentration $c(\text { TAPO })_{0}=7.1 \cdot 10^{-6} \mathrm{~mol} \cdot \mathrm{L}^{-1}$. Die Reaktionstemperatur lag zwischen 144 und $188{ }^{\circ} \mathrm{C}$. Der Stoffmengenanteil an Propionaldehyd betrug 0.0032. Als $T_{\text {opt }}$ wurde $178 \pm 5^{\circ} \mathrm{C}$ bestimmt. Der Peroxid-induzierte Ethenumsatz ist in Abbildung 4.27 gezeigt.

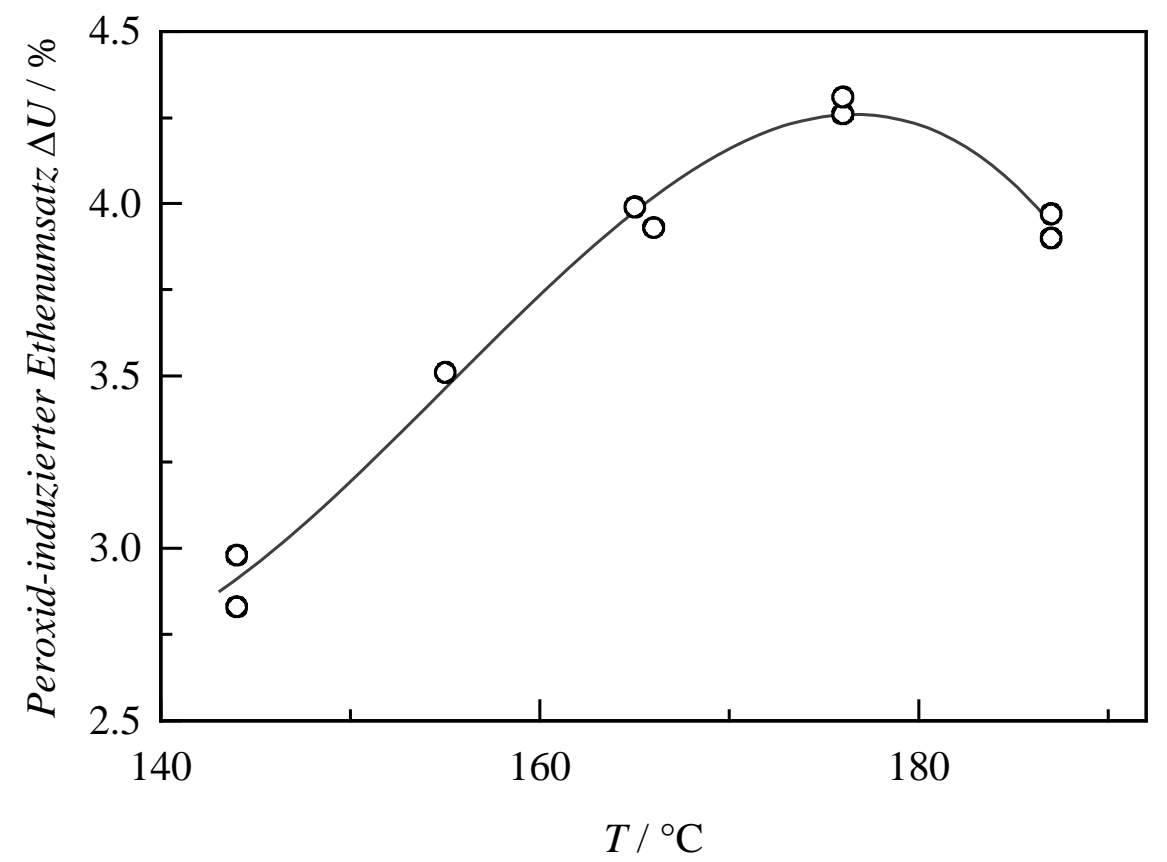

Abb. 4.27: Temperaturabhängigkeit des Peroxid-induzierten Ethenumsatzes für EthenHomopolymerisation mit TAPO als Initiator;

$p=2000 \mathrm{bar}, m_{E}=706 \mathrm{~g} \cdot \mathrm{h}^{-1} ; \mathrm{c}(\mathrm{TAPO})_{0}=7.1 \cdot 10^{-6} \mathrm{~mol} \cdot \mathrm{L}^{-1}$.

Die Temperaturabhängigkeit der Initiatoreffektivität ist in Abbildung 4.28 zu sehen. Der Mittelwert der erhaltenden Effektivitäten ist 0.61. 


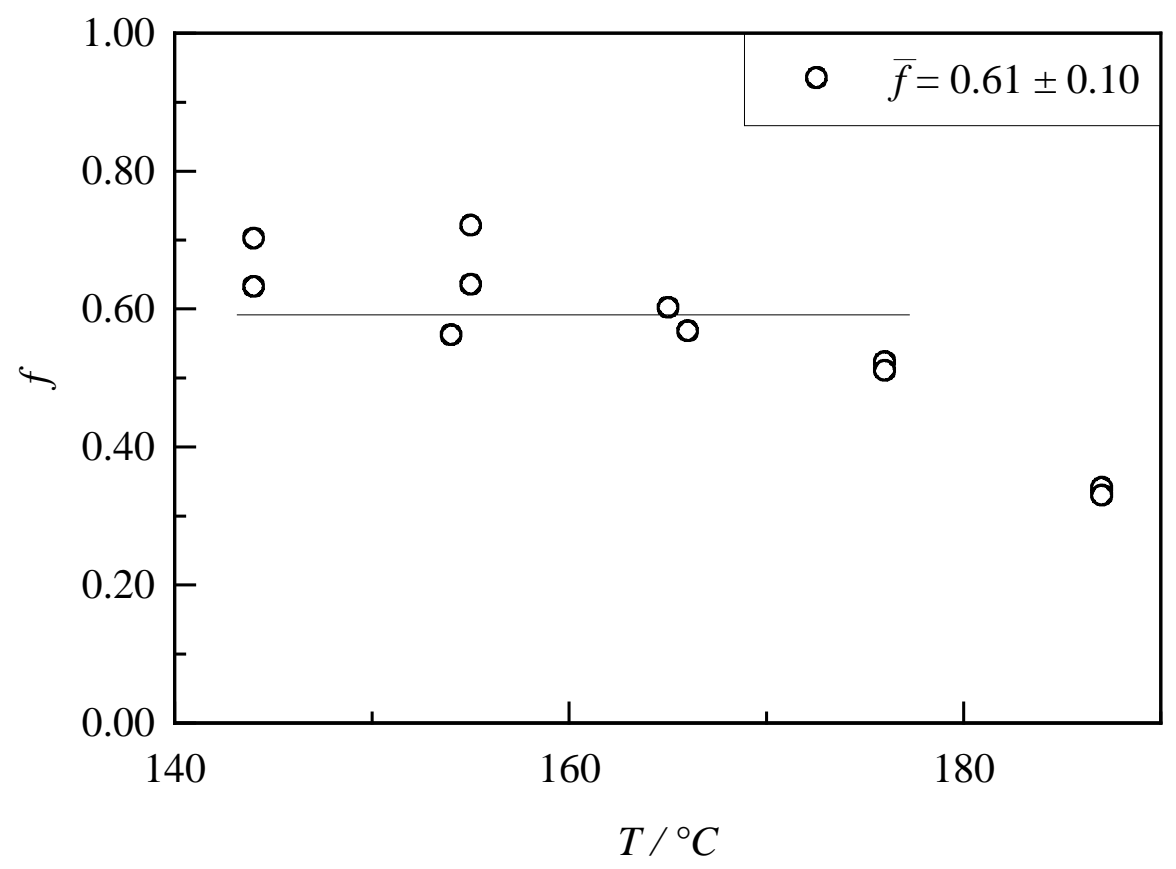

Abb. 4.28: Temperaturabhängigkeit der Initiatoreffektivität für TAPO. $p=2000 \mathrm{bar}, m_{E}=706 \mathrm{~g} \cdot \mathrm{h}^{-1} ; \mathrm{c}(\mathrm{TAPO})_{0}=7.1 \cdot 10^{-6} \mathrm{~mol} \cdot \mathrm{L}^{-1}$.

\subsubsection{Initiierungsexperiment mit TAPP als Initiator}

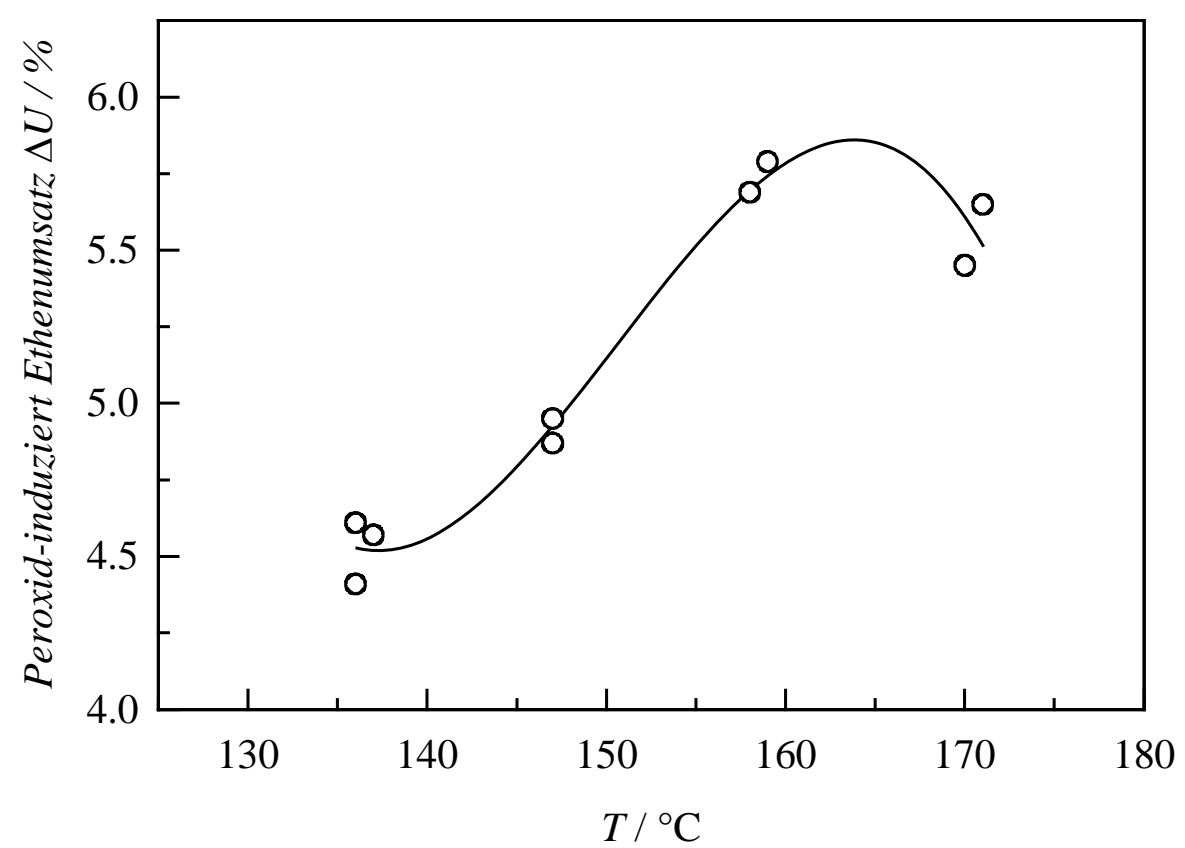

Abb. 4.29: Temperaturabhängigkeit des Peroxid-induzierten Ethenumsatzes für EthenHomopolymerisation mit TAPP als Initiator;

$p=2000 \mathrm{bar}, m_{E}=706 \mathrm{~g} \cdot \mathrm{h}^{-1} ; \mathrm{c}(\mathrm{TAPP})_{0}=2.3 \cdot 10^{-5} \mathrm{~mol} \cdot \mathrm{L}^{-1}$. 
In Abbildung 4.29 ist der Peroxid-induzierte Ethenumsatz mit TAPP als Initiator gegen die Temperatur aufgetragen. Die Initiatorkonzentration betrug $c(\mathrm{TAPP})_{0}=2.3 \cdot 10^{-5} \mathrm{~mol} \cdot \mathrm{L}^{-1}$. Die Reaktionstemperatur lag zwischen 137 und $171^{\circ} \mathrm{C}$. Der Stoffmengenanteil an Propionaldehyd betrug 0.0032. Als optimale Temperatur wurde $164 \pm 5^{\circ} \mathrm{C}$ bestimmt.

Die erhaltene Temperaturabhängigkeit der Initiatoreffektivität ist in Abbildung 4.30 dargestellt. Der Mittelwert der Initiatoreffektivitäten beträgt 0.48.

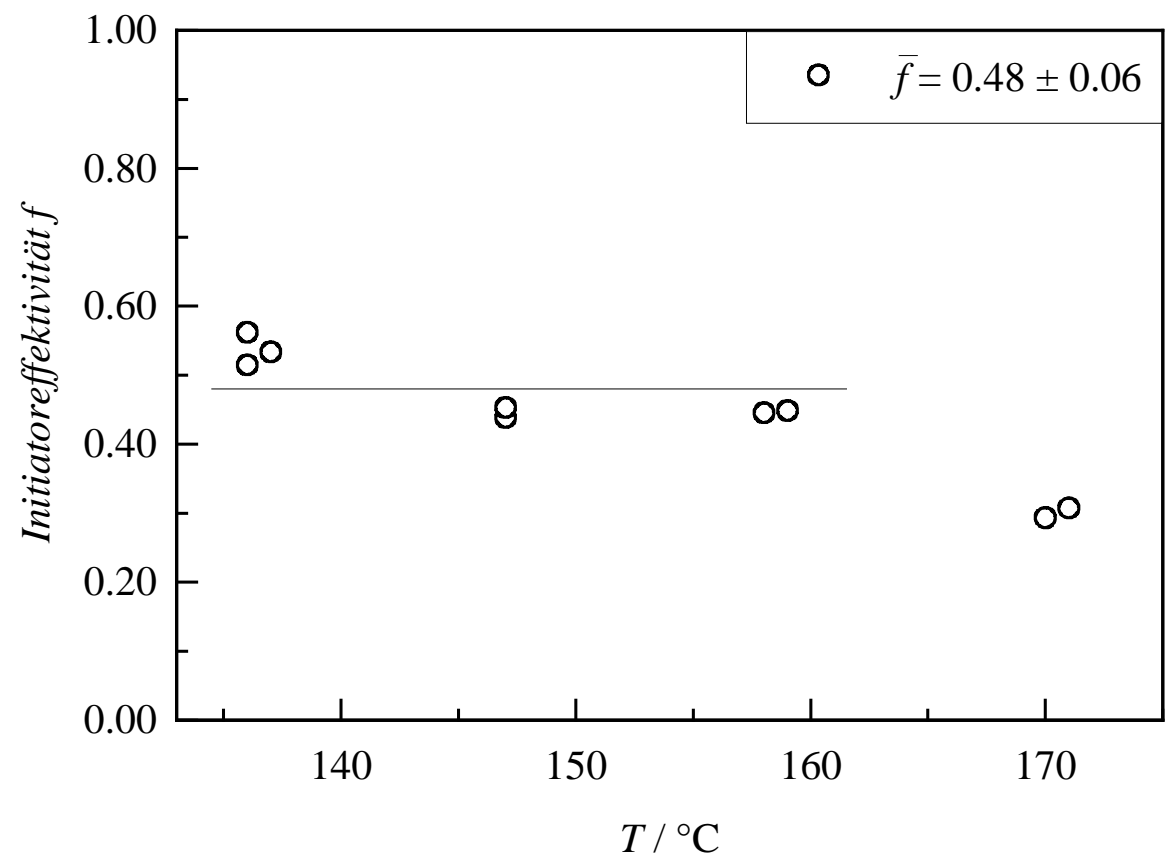

Abb. 4.30: Temperaturabhängigkeit der Initiatoreffektivität für TAPP. $p=2000 \mathrm{bar}, m_{E}=706 \mathrm{~g} \cdot \mathrm{h}^{-1} ; \mathrm{c}(\mathrm{TAPP})_{0}=2.3 \cdot 10^{-5} \mathrm{~mol} \cdot \mathrm{L}^{-1}$.

\subsection{1,1,2,2-Tetramethylpropylperoxypivalat}

Im Rahmen dieser Arbeit wurde 1,1,2,2-Tertramethylpropylperoxypivalat (TMPPP) untersucht. Die Strukturformel von TMPPP ist in Abbildung $4.31 \mathrm{zu}$ sehen. Der strukturelle Unterschied zu TAPP ist, dass die endständig Methylgruppe auf der Alkoholseite durch eine tert-Butylgruppe ersetzt worden ist. Dadurch wird der Zerfall von TMPPP gegenüber TAPP beschleunigt. Die Zerfallskinetik wurde von VöGELE untersucht [19]. Die Gleichung zur Bestimmung der Geschwindigkeitskonstante des thermischen Zerfalls ist unten aufgeführt.

$$
k_{\mathrm{obs}}=7.98 \cdot 10^{13} \mathrm{~s}^{-1} \exp -\left(\frac{115.7 \mathrm{~kJ} \mathrm{~mol}^{-1}+1.8 \mathrm{~cm}^{3} \mathrm{~mol}^{-1} \cdot p}{R T}\right)
$$




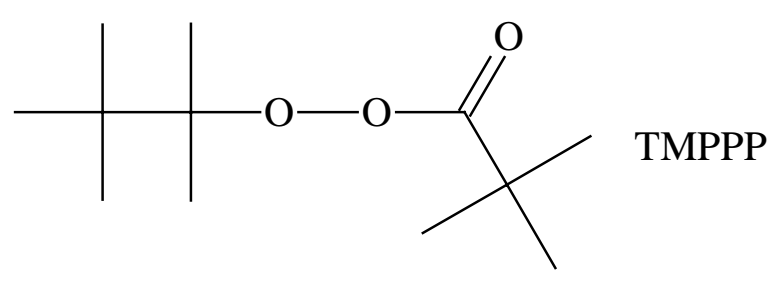

Abb. 4.31: Strukturformel von 1,1,2,2-Tetramethylpropylperoxypivalat.

In Abbildung 4.32 ist der TMPPP induzierte Ethenumsatz in der Hochdruckpolymerisation gegen die Temperatur aufgetragen. Die Initiatorkonzentration betrug $c(\mathrm{TMPPP})_{0}=$ 8.8 $\cdot 10^{-6} \mathrm{~mol} \cdot \mathrm{L}^{-1}$. Die Reaktionstemperatur lag zwischen 129 und $156{ }^{\circ} \mathrm{C}$. Der Stoffmengenanteil an Propionaldehyd betrug 0.0032. Die optimale Temperatur lag bei $144 \pm 5^{\circ} \mathrm{C}$.

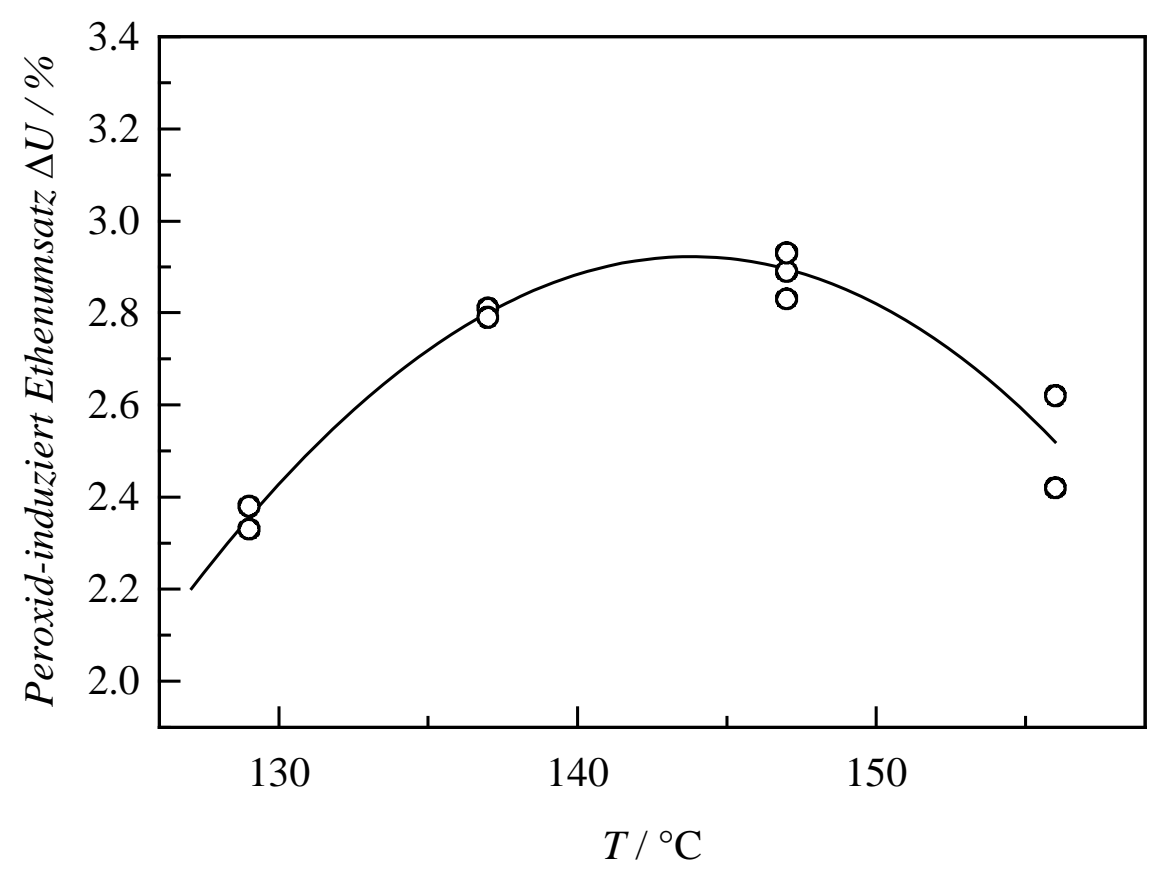

Abb. 4.32: Temperaturabhängigkeit des Peroxid-induzierten Ethenumsatzes für EthenHomopolymerisation mit TMPPP als Initiator;

$p=2000 \mathrm{bar}, m_{E}=706 \mathrm{~g} \cdot \mathrm{h}^{-1} ; \mathrm{c}(\mathrm{TMPPP})_{0}=8.8 \cdot 10^{-6} \mathrm{~mol} \cdot \mathrm{L}^{-1}$

In Abbildung 4.33 ist die Initiatoreffektivität gegen die Temperatur aufgetragen. Es wird im Mittel eine Initiatoreffektivität von 0.50 erhalten. 


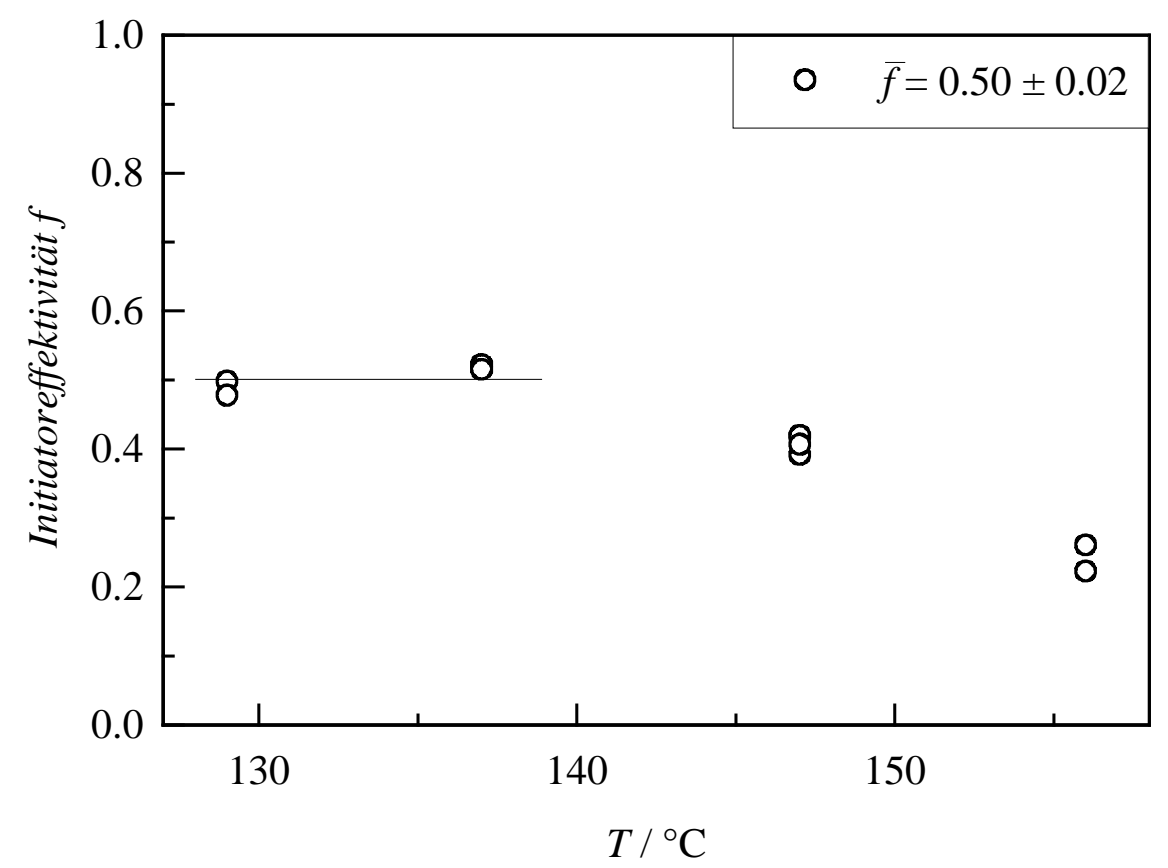

Abb. 4.33: Temperaturabhängigkeit der Initiatoreffektivität für TMPPP. $p=2000 \mathrm{bar}, m_{E}=706 \mathrm{~g} \cdot \mathrm{h}^{-1} ; \mathrm{c}(\mathrm{TMPPP})_{0}=8.8 \cdot 10^{-6} \mathrm{~mol} \cdot \mathrm{L}^{-1}$.

\subsection{1,1,3,3-Tetramethylbutylperoxyester}

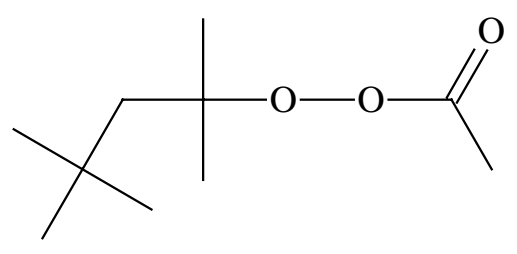

TMBPA
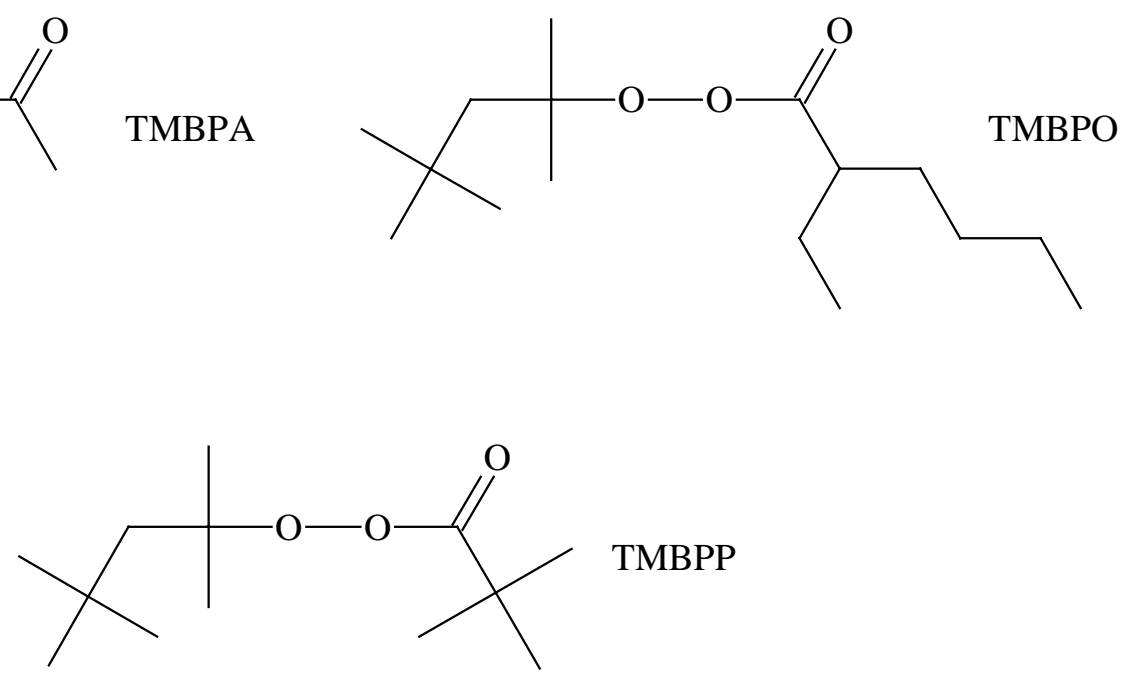

Abb. 4.34: Strukturformeln der untersuchten 1,1,3,3-Tetramethylbutylperoxyester.

In diesem Abschnitt werden die Initiierungsexperimente von drei Tetramethylbutylperoxyestern vorgestellt. Es wurde untersucht 1,1,3,3Tetramethylbutylperoxyacetat (TMBPA), 1,1,3,3-Tetramethylbutylperoxy-2-ethylhexanoat 
(TMBPO) und 1,1,3,3-Tetramethylbutylperoxypivalat (TMBPP) untersucht. Die Strukturformeln sind in Abbildung $4.34 \mathrm{zu}$ sehen. Es handelt sich wiederum um einen primären (TMBPA), einen sekundären (TMBPO) und einen tertiären (TMBPP) Peroxyester hinsichtlich des $\alpha$-C-Atoms der Säureseite. Es soll - wie für die vorangegangenen Peroxyester - untersucht werden, wie dieser strukturelle Unterschied die Initiatoreffektivität beeinflusst. Die Zerfallskinetik wurde von VöGELE untersucht [19]. In Tabelle 4.3 sind die Aktivierungsparameter des Geschwindigkeitskoeffizienten des thermischen Zerfalls aufgeführt.

\begin{tabular}{cccc}
\hline Peroxid & $\boldsymbol{E}_{\mathbf{A}}(\mathbf{0} \mathbf{b a r}) / \mathbf{k J} \cdot \mathbf{m o l}^{-\mathbf{1}}$ & $\boldsymbol{A} / \mathbf{s}^{-\mathbf{1}}$ & $\Delta \boldsymbol{V}^{\neq} / \mathbf{c m}^{\mathbf{3}} \cdot \mathbf{m o l}^{-\mathbf{1}}$ \\
\hline TMBPA & 138.8 & $1.55 \cdot 10^{15}$ & 12.8 \\
TMBPO & 128.3 & $7.49 \cdot 10^{14}$ & 4.0 \\
TMBPP & 118.4 & $3.16 \cdot 10^{14}$ & 1.2 \\
\hline
\end{tabular}

Tab.4.3: Aktivierungsparameter des Geschwindigkeitskoeffizienten des thermischen Zerfalls für die untersuchten 1,1,3,3-Tetramethylbutylperoxyester [19].

\subsubsection{Initiierungsexperimente mit 1,1,3,3-Tetramethylbutylperoxyacetat}

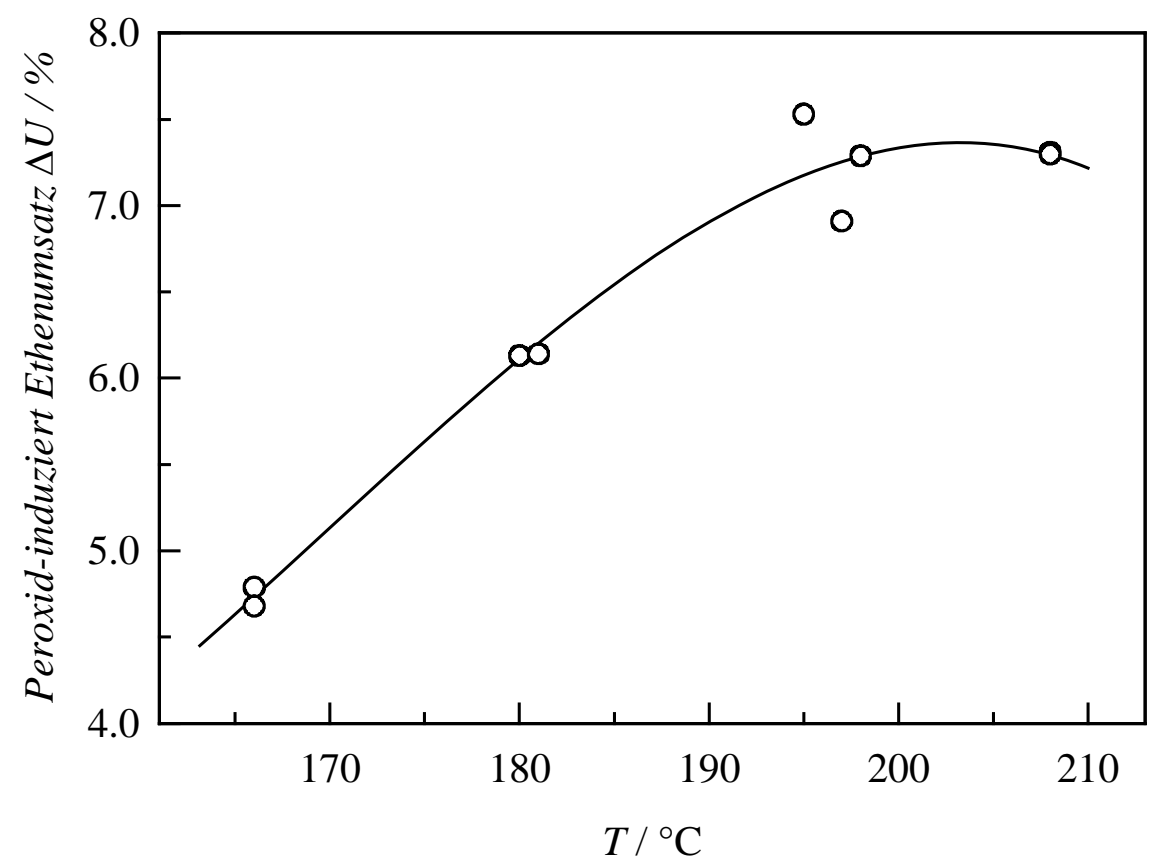

Abb. 4.35: Temperaturabhängigkeit des Peroxid-induzierten Ethenumsatzes für EthenHomopolymerisation mit TMBPA als Initiator; $p=2000 \mathrm{bar}, m_{E}=706 \mathrm{~g} \cdot \mathrm{h}^{-1} ; \mathrm{c}(\mathrm{TMBPA})_{0}=8.9 \cdot 10^{-6} \mathrm{~mol} \cdot \mathrm{L}^{-1}$. 
In Abbildung 4.35 ist der durch TMBPA induzierte Ethenumsatz in der Hochdruckpolymerisation gegen die Temperatur aufgetragen. Die Reaktionstemperatur lag zwischen 163 und $208{ }^{\circ} \mathrm{C}$. Als Initiatorkonzentration von TMBPA wurde $c(\mathrm{TMBPA})_{0}=8.9 \cdot 10^{-6} \mathrm{~mol} \cdot \mathrm{L}^{-1}$ verwendet. Der Stoffmengenanteil an Propionaldehyd betrug 0.0032. $T_{\text {opt }}$ lag bei $204 \pm 5{ }^{\circ} \mathrm{C}$.

Die resultierende Initiatoreffektivität ist in Abbildung $4.35 \mathrm{zu}$ sehen. Es wird im Mittel eine Initiatoreffektivität von 0.76 erhalten.

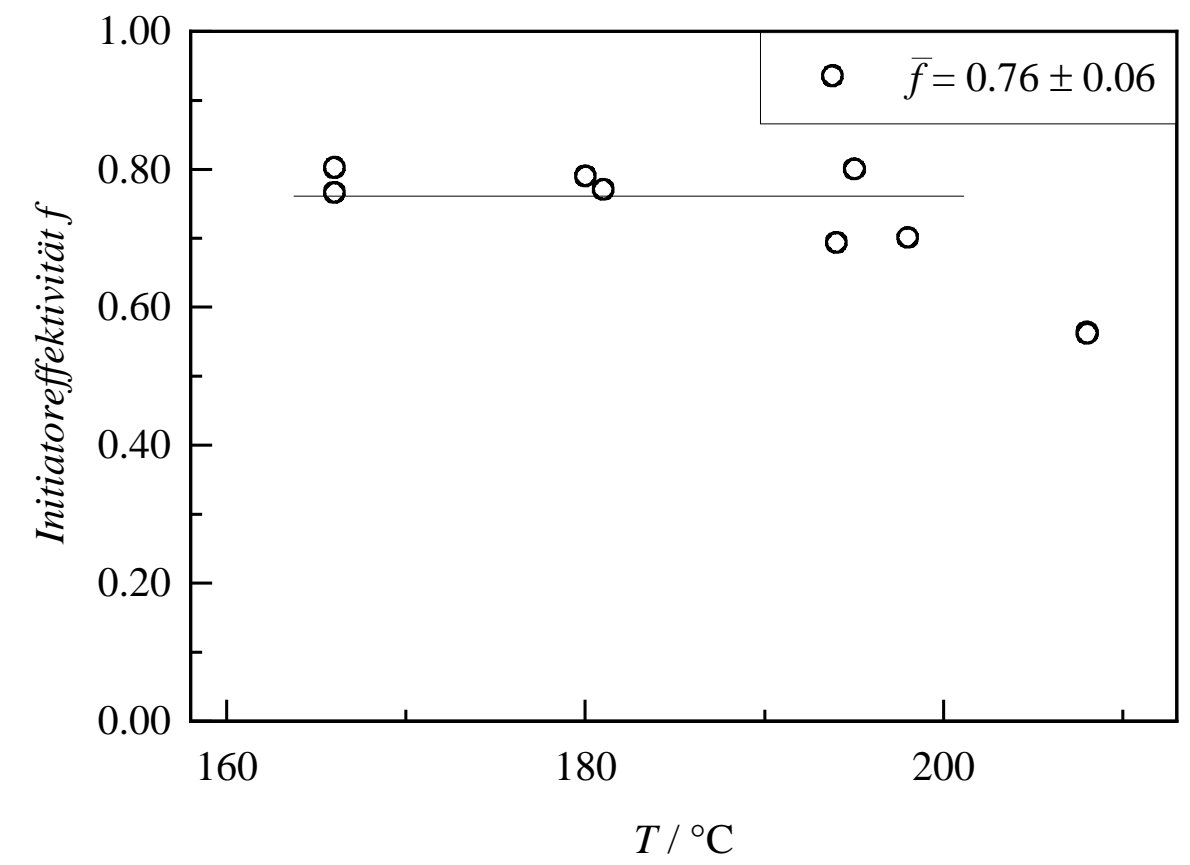

Abb. 4.36: Temperaturabhängigkeit der Initiatoreffektivität für TMBPA. $p=2000 \mathrm{bar}, m_{E}=706 \mathrm{~g} \cdot \mathrm{h}^{-1} ; \mathrm{c}(\mathrm{TMBPA})_{0}=8.9 \cdot 10^{-6} \mathrm{~mol} \cdot \mathrm{L}^{-1}$.

\subsubsection{Initiierungsexperimente mit 1,1,3,3-Tetramethylbutylperoxy-2-ethyl- hexanoat}

Die Temperaturabhängigkeit des durch TMBPO induzierten Ethenumsatzes in der Hochdruckpolymerisation wurde bereits in Abbildung 4.2 im Kapitel 4.4 dargestellt. Es wurden zwei Experimente durchgeführt. Die Initiatorkonzentration der ersten Messung betrug $c(\text { TMBPO })_{0}=7.0 \cdot 10^{-6} \mathrm{~mol} \cdot \mathrm{L}^{-1}$, die der zweiten war doppelt so hoch mit $c(\mathrm{TMBPO})_{0}=$ $1.4 \cdot 10^{-5} \mathrm{~mol} \cdot \mathrm{L}^{-1}$. Die Reaktionstemperatur lagen zwischen 145 und $188{ }^{\circ} \mathrm{C}$ für die erste und zwischen 138 und $186{ }^{\circ} \mathrm{C}$ für die zweite Messung. Der Stoffmengenanteil an Propionaldehyd betrug bei beiden Experimenten 0.0032. Die optimale Temperatur lag bei $177 \pm 5^{\circ} \mathrm{C}$ bzw. bei $182 \pm 5^{\circ} \mathrm{C}$. 
In Abbildung 4.37 ist für beide Experimente die Temperaturabhängigkeit der Initiatoreffektivität gezeigt. Die offenen Symbole sind die Werte, die für $c(\mathrm{TMBPO})_{0}=$ $1.4 \cdot 10^{-5} \mathrm{~mol} \cdot \mathrm{L}^{-1}$ erhalten worden sind. Die gefüllten Symbole stellen die Werte für $c(\text { TMBPO })_{0}=7.0 \cdot 10^{-6} \mathrm{~mol} \cdot \mathrm{L}^{-1}$ dar. Die Effektivitäten liegen zwischen 0.56 und 0.65 und sind innerhalb Fehlergrenzen identisch.

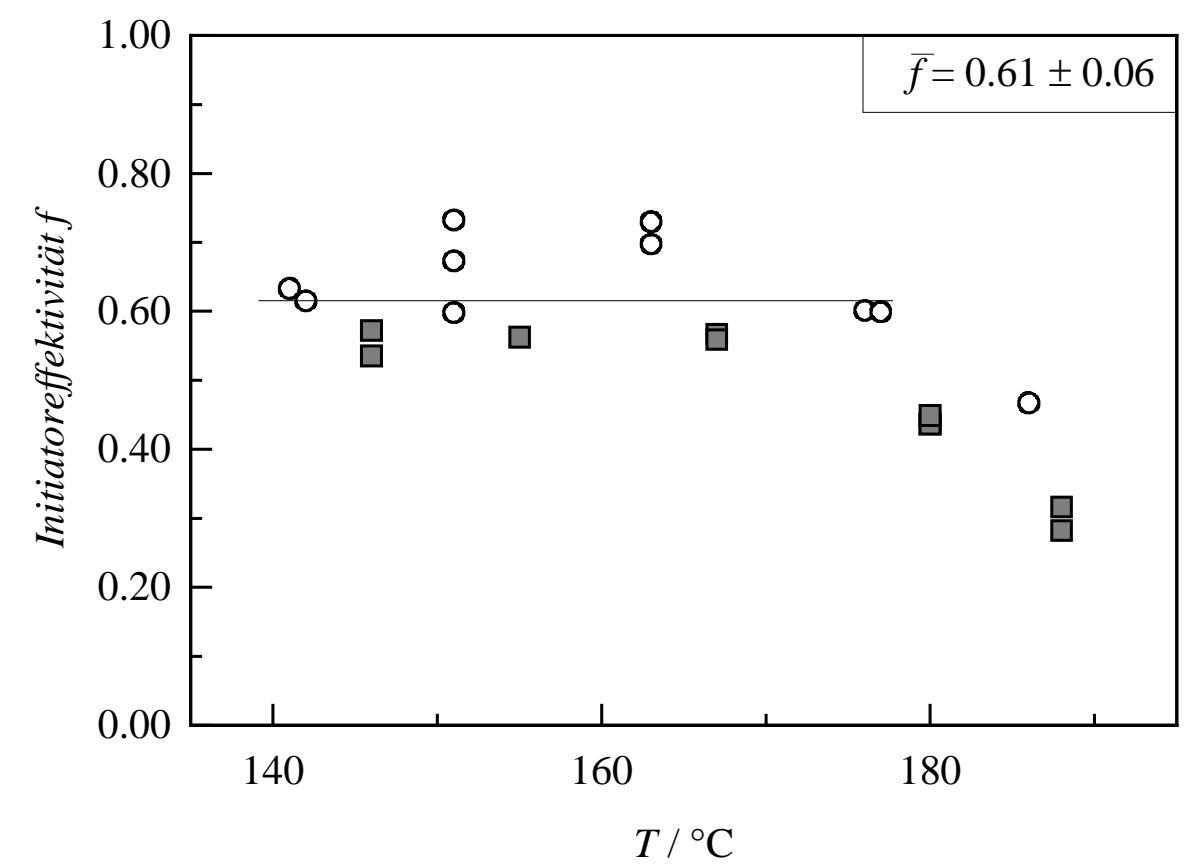

Abb. 4.37: Temperaturabhängigkeit der Initiatoreffektivität für TMBPO. Offene Symbole $c(\text { TMBPO })_{0}=1.4 \cdot 10^{-5} \mathrm{~mol} \cdot \mathrm{L}^{-1}$; gefüllte Symbole $c(\mathrm{TMBPO})_{0}=7.0 \cdot 10^{-6} \mathrm{~mol} \cdot \mathrm{L}^{-1}$; $p=2000 \mathrm{bar}, m_{E}=706 \mathrm{~g} \cdot \mathrm{h}^{-1}$.

\subsubsection{Initiierungsexperiment mit 1,1,3,3-Tetramethylbutylperoxypivalat}

Bei diesem Experiment wurde nur bei zwei Temperaturen unterhalb von $T_{\text {opt }}$ gemessen, weil unterhalb von $130{ }^{\circ} \mathrm{C}$ das Ethen/Polymergemisch zweiphasig werden kann. Der TMBPPinduzierte Ethenumsatz in der Hochdruckpolymerisation ist in Abbildung 4.38 zu sehen. Die Initiatorkonzentration betrug $c(\mathrm{TMBPP})_{0}=2.3 \cdot 10^{-5} \mathrm{~mol} \cdot \mathrm{L}^{-1}$. Die Reaktionstemperatur lagen zwischen 130 und $153{ }^{\circ} \mathrm{C}$. Der Stoffmengenanteil an Propionaldehyd betrug 0.0032. Die optimale Temperatur lag bei $150 \pm 5^{\circ} \mathrm{C}$. 


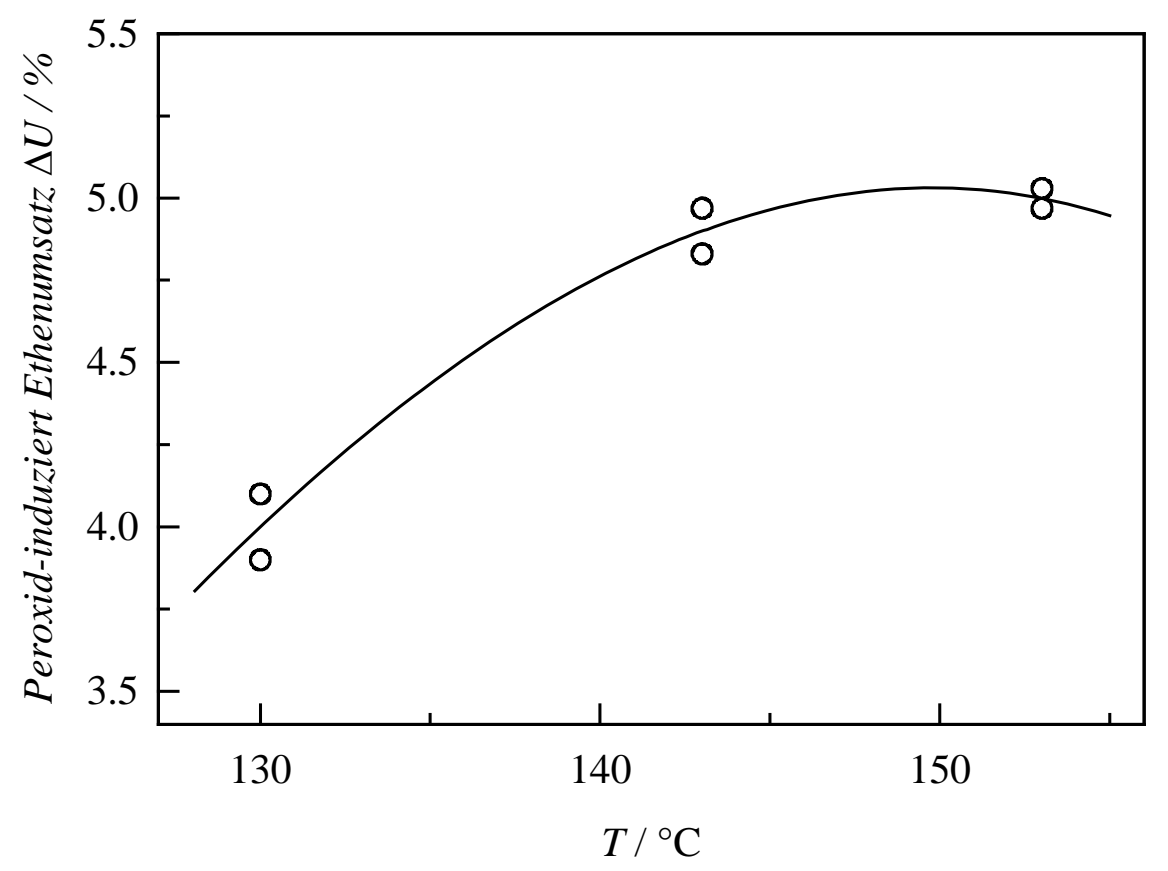

Abb. 4.38: Temperaturabhängigkeit des Peroxid-induzierten Ethenumsatzes für EthenHomopolymerisation mit TMBPP als Initiator;

$p=2000 \mathrm{bar}, m_{E}=706 \mathrm{~g} \cdot \mathrm{h}^{-1} ; \mathrm{c}(\mathrm{TMBPP})_{0}=2.3 \cdot 10^{-5} \mathrm{~mol} \cdot \mathrm{L}^{-1}$.

In Abbildung 4.39 ist die Initiatoreffektivität von TMBPP in Abhängigkeit von der Temperatur zu sehen. $f$ betrug im Mittel 0.50.

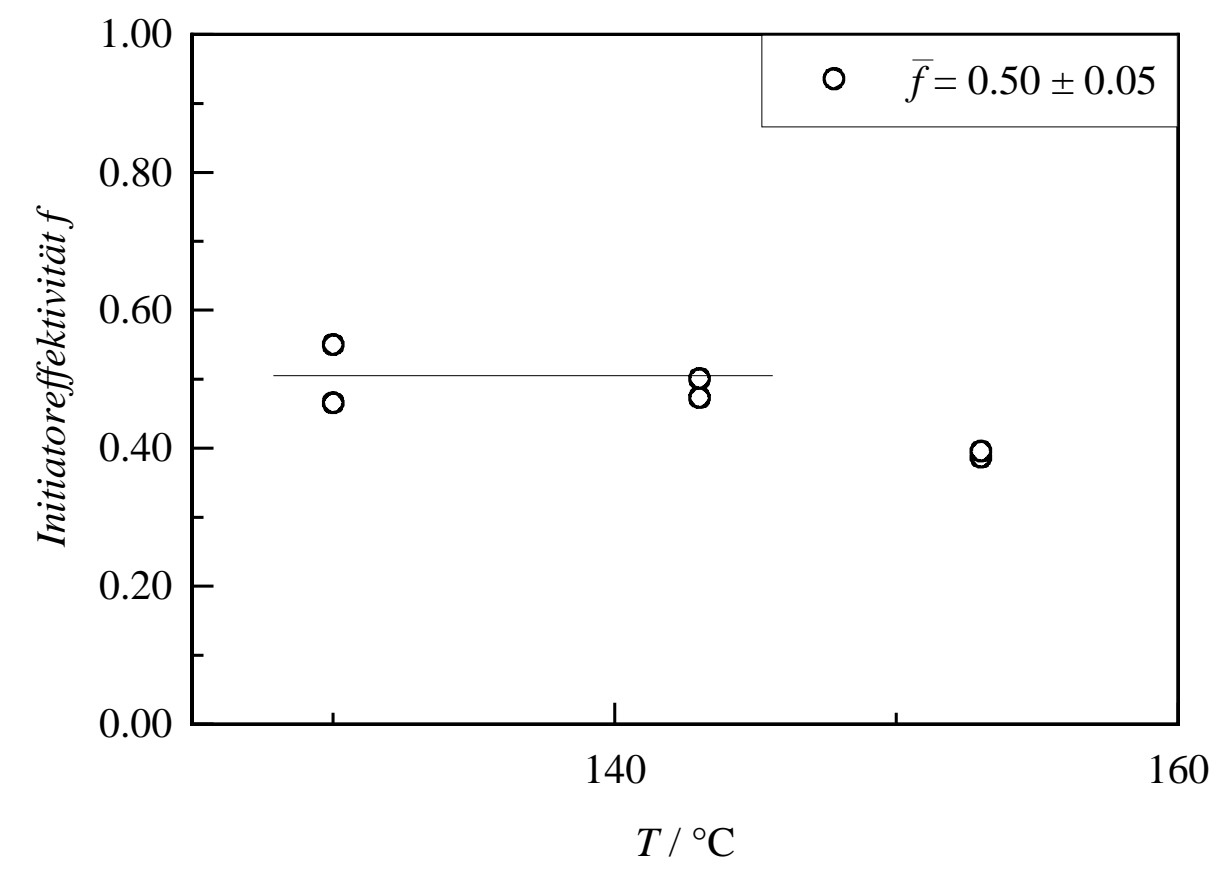

Abb. 4.39: Temperaturabhängigkeit der Initiatoreffektivität für TMBPP. $p=2000 \mathrm{bar}, m_{E}=706 \mathrm{~g} \cdot \mathrm{h}^{-1} ; \mathrm{c}(\mathrm{TMBPP})_{0}=2.3 \cdot 10^{-5} \mathrm{~mol} \cdot \mathrm{L}^{-1}$. 


\subsection{Druckabhängigkeit der Initiatoreffektivität}

Da die Ethen-Hochdruckpolymerisationen im weiten Zustandsbereich durchgeführt werden, erschien es sehr wichtig, hierzu auch Experimente durchzuführen. Als Modellverbindungen wurden tert-Butylperoxypivalat (TBPP), tert-Butylperoxy-2-ethylhexanoat (TBPO) und Ditert-butylperoxid (DTBP) ausgewählt. Die Strukturformeln sind in Abbildung 4.40 gezeigt.

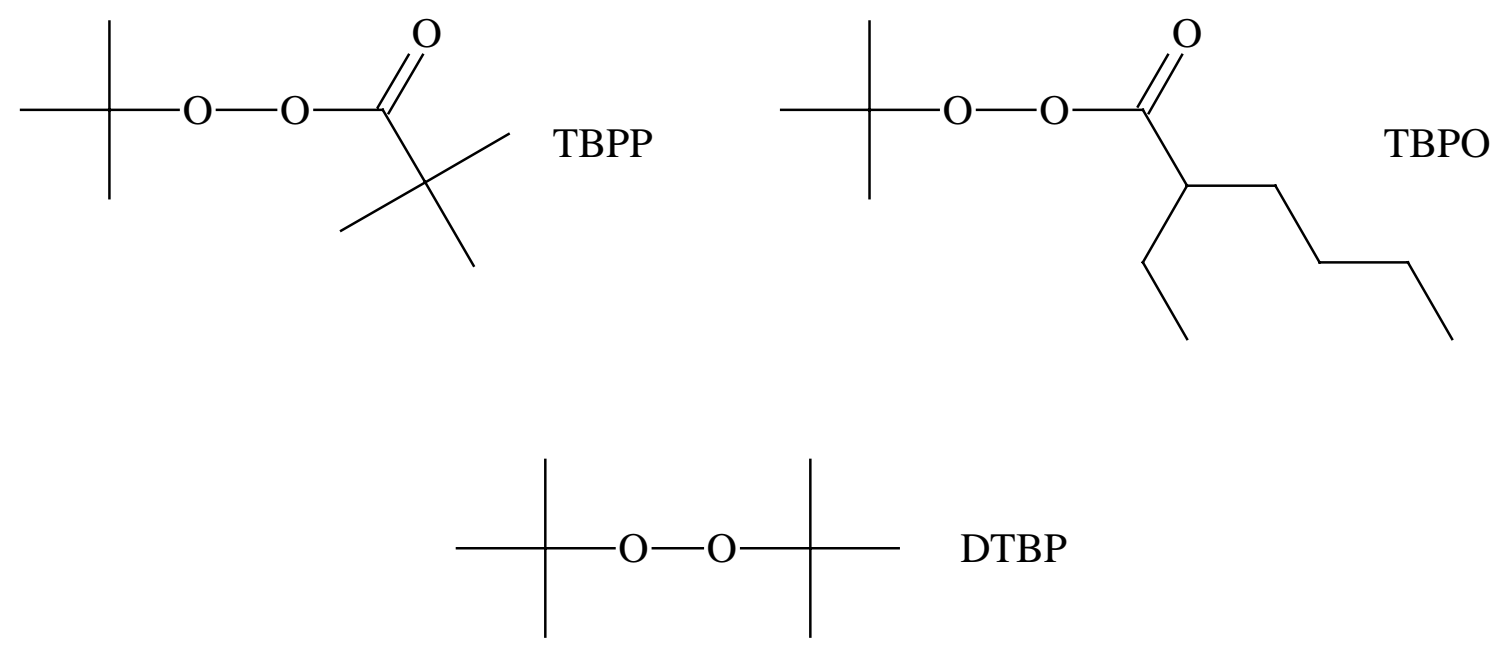

Abb. 4.40: Strukturformeln der untersuchten Peroxide.

Für alle drei Initiatoren gibt es Literaturwerte für die Initiatoreffektivität bei 2000 bar [2]. Die Druck- und Temperaturabhängigkeit der thermischen Zerfallsgeschwindigkeitskonstanten für die untersuchten Peroxide ist ebenfalls bekannt [18, 12, 13].

Bei einem Druck unterhalb von 1500 bar und einer Temperatur von $150{ }^{\circ} \mathrm{C}$ wird das Polymer/Ethen-Gemisch inhomogen [21]. Es wurde daher nur bei Drücken oberhalb von 1600 bar gemessen. Die in Kapitel 3 beschriebene Apparatur ist bis zu einem Druck von 3000 bar zugelassen. Um das Material nicht an seine Grenzen zu belasten, wurde bei 1600, 1800, 2000, 2250 und 2450 bzw. 2500 bar gemessen. Bei den Experimenten wurde jeweils dieselbe Manteltemperatur eingestellt. Doch aufgrund der Reaktionswärme heizte sich das Reaktionsgemisch je nach Ethenumsatz unterschiedlich auf, so dass sich die Reaktionstemperaturen leicht unterschieden. Für die Berechnung der Initiatoreffektivität wird Gleichung 4.15 benutzt, da für alle darin enthaltenen Geschwindigkeitskonstanten die Druckabhängigkeit bekannt ist. Die unterschiedlichen Reaktionstemperaturen werden berücksichtigt. 


\subsubsection{Initiierungsexperimente mit Di-tert-butylperoxid}

In Abbildung 4.41 ist die Druckabhängigkeit des DTBP induzierten Ethenumsatzes in der Hochdruckpolymerisation zu sehen. Die eingesetzte Initiatorkonzentration betrug $c(\mathrm{DTBP})_{0}=$ $2.8 \cdot 10^{-6} \mathrm{~mol} \cdot \mathrm{L}^{-1}$. Die Reaktionstemperatur lag zwischen 199 und $203^{\circ} \mathrm{C}$. Der Stoffmengenanteil an Propionaldehyd betrug 0.0032. Mit dem Druck steigt der Ethenumsatz stetig an, da zum einen die Ethenkonzentration und zum anderen $k_{\mathrm{p}} / k_{\mathrm{t}}^{0.5}$ mit dem Druck größer wird.

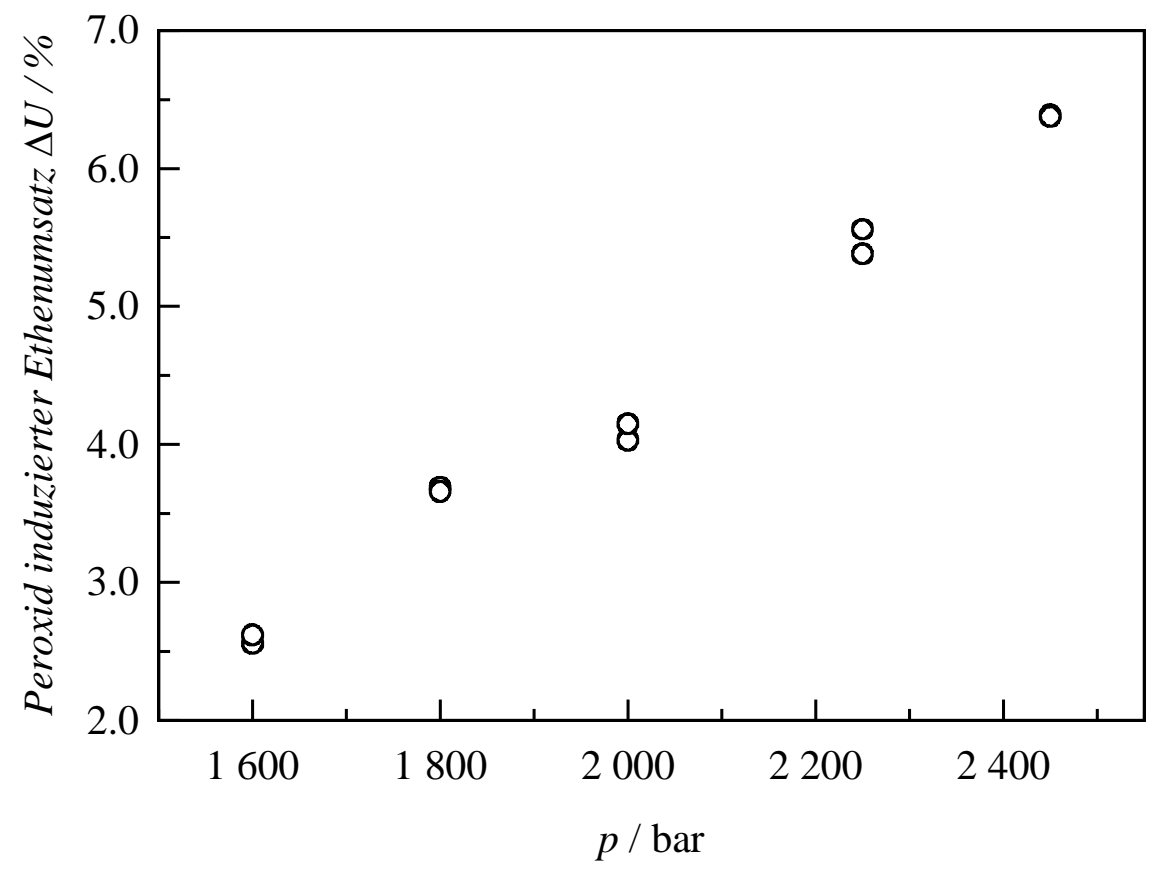

Abb. 4.41: Druckabhängigkeit des Peroxid-induzierten Ethenumsatzes für Ethen-Homopolymerisation mit DTBP als Initiator.

$T=199 \mathrm{bis} 20{ }^{\circ} \mathrm{C}, \mathrm{m}_{\mathrm{E}}=706 \mathrm{~g} \cdot \mathrm{h}^{-1} ; \mathrm{c}(\mathrm{DTBP})_{0}=2.8 \cdot 10^{-6} \mathrm{~mol} \cdot \mathrm{L}^{-1}$.

In Abbildung 4.42 ist die resultierende Initiatoreffektivität von DTBP in Abhängigkeit vom Druck gezeigt. Es ist nur ein leichter Abfall zu erkennen. Auf Grund der Streuung der Datenpunkte ist eine gesicherte Aussage, ob f(DTBP) druckabhängig ist, nicht zu treffen. Die Effektivität liegt bei 1800 bar mit 1.11 über eins. Der bei 2000 bar erhaltende Punkt ist mit 0.92 unter dem Literaturwert von eins [2]. Der Abfall von 0.97 bei 1600 bar auf 0.92 bei 2450 bar, dies entspricht $(\Delta f / \Delta p) \cdot 1000 \mathrm{bar}=-0.06$, ist sehr gering. 


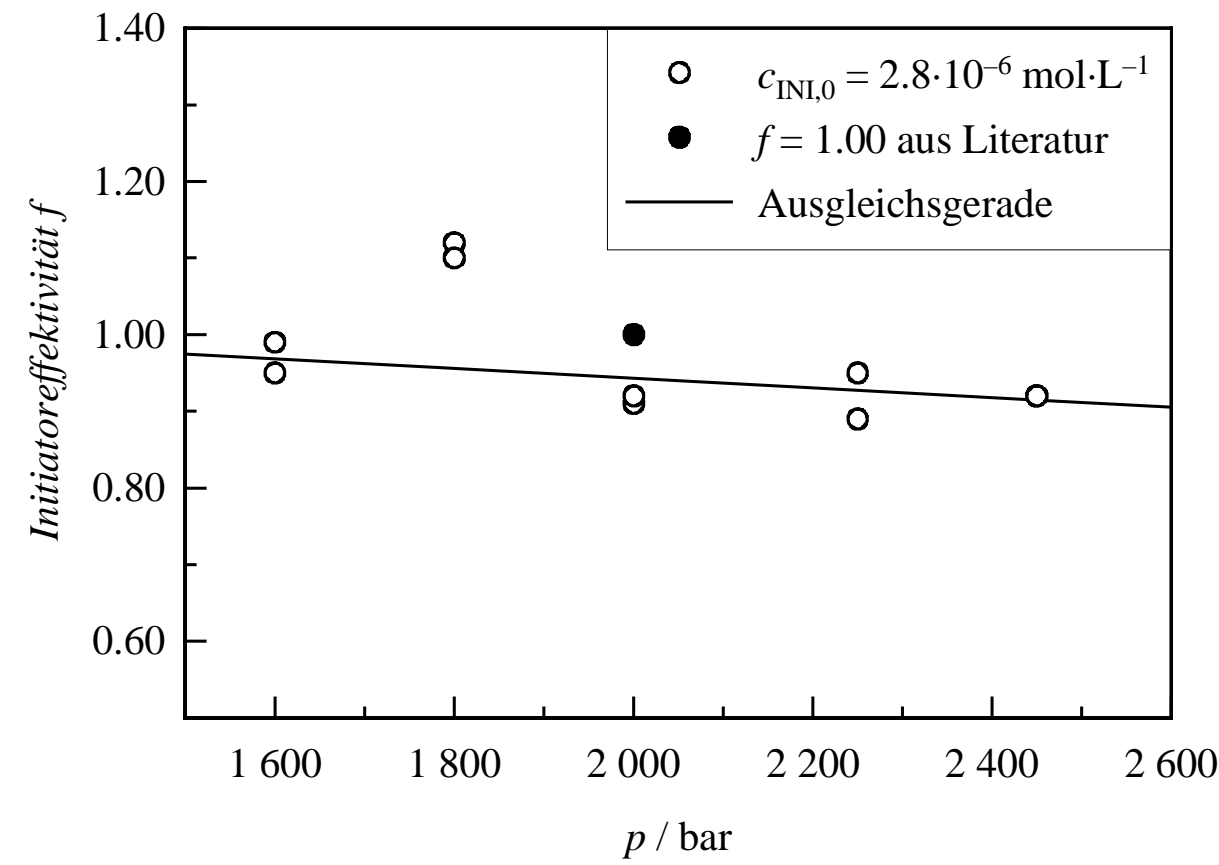

Abb. 4.42: Druckabhängigkeit der Initiatoreffektivität für DTBP.

$T=199$ bis $203{ }^{\circ} \mathrm{C}, m_{E}=706 \mathrm{~g} \cdot \mathrm{h}^{-1} ; \mathrm{c}(\mathrm{DTBP})_{0}=2.8 \cdot 10^{-6} \mathrm{~mol} \cdot \mathrm{L}^{-1}$.

\subsubsection{Initiierungsexperimente mit tert-Butylperoxypivalat}

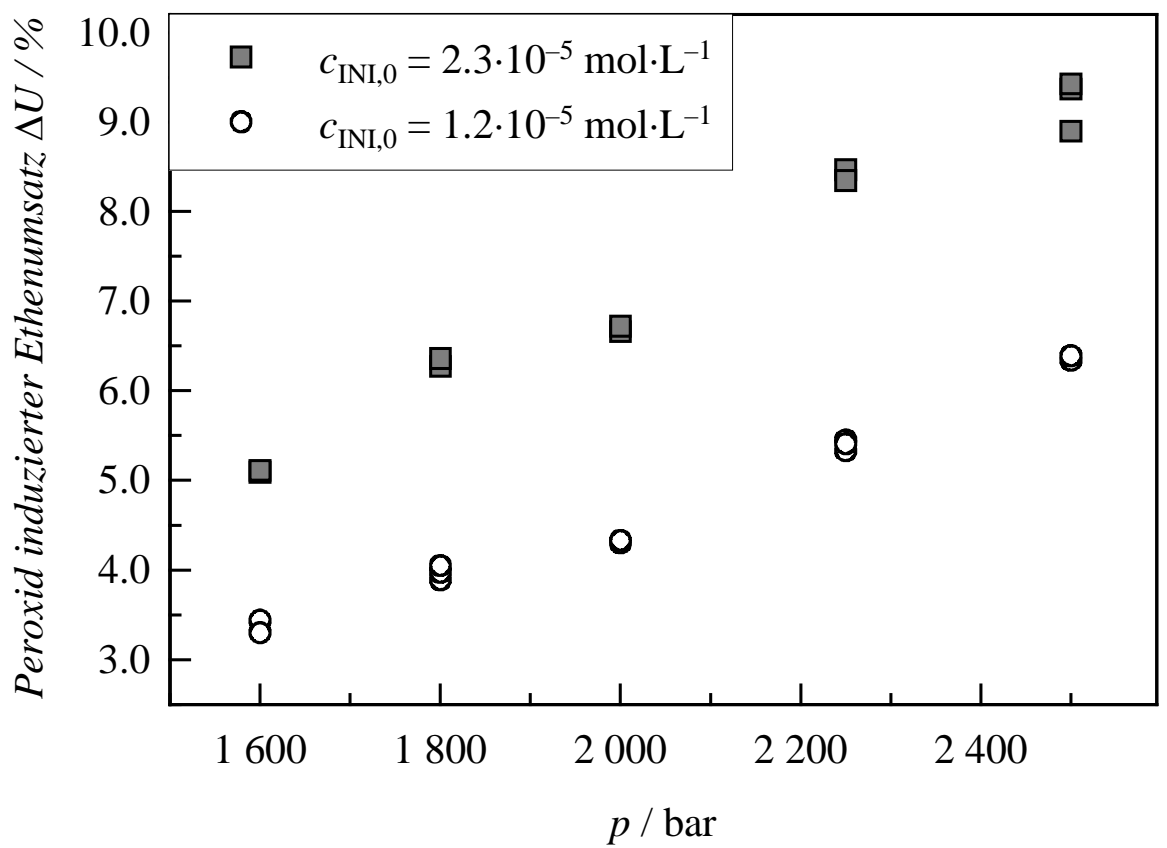

Abb. 4.43: Druckabhängigkeiten des Peroxid-induzierten Ethenumsatzes für EthenHomopolymerisationen mit TBPP als Initiator; offene Kreise $c(T B P P)_{0}=$ $1.2 \cdot 10^{-5} \mathrm{~mol} \cdot \mathrm{L}^{-1}$; gefüllte Quadrate $c(T B P P)_{0}=2.3 \cdot 10^{-5} \mathrm{~mol} \cdot \mathrm{L}^{-1}$;

$T=156$ bis $162{ }^{\circ} \mathrm{C}$ (offene Kreise), $T=163$ bis $172{ }^{\circ} \mathrm{C}$ (gefüllte Quadrate), $m_{E}=706 \mathrm{~g} \cdot \mathrm{h}^{-1}$. 
In der Abbildung 4.43 ist die Druckabhängigkeit des TBPP-induzierten Ethenumsatzes in der Hochdruckpolymerisation $\mathrm{zu}$ sehen. Es wurden Experimente bei zwei Initiatorkonzentrationen durchgeführt.

Die Initiatorkonzentration der ersten Messung betrug $c(\text { TBPP })_{0}=1.2 \cdot 10^{-5} \mathrm{~mol} \cdot \mathrm{L}^{-1}$. Die Ethenumsätze sind in Abbildung 4.43 mit offenen Kreisen dargestellt. Die Reaktionstemperatur lag zwischen 156 und $162{ }^{\circ} \mathrm{C}$. Der Stoffmengenanteil an Propionaldehyd betrug bei beiden Experimenten 0.0032. Bei der zweiten Messung wurde eine Initiatorkonzentration von $c(\text { TBPP })_{0}=2.3 \cdot 10^{-5} \mathrm{~mol} \cdot \mathrm{L}^{-1}$ verwendet. Die Reaktionstemperatur lag zwischen 163 und $172{ }^{\circ} \mathrm{C}$. Die daraus resultierenden Ethenumsätze sind in Abbildung 4.43 als gefüllte Quadrate zu sehen.

In Abbildung 4.44 ist für beide Messungen die Druckabhängigkeit der Initiatoreffektivität gezeigt. Die erhaltenden Effektivitäten sind für beide Experimente nahezu deckungsgleich und stark druckabhängig. $f$ sinkt von 0.59 bei 1600 bar auf 0.23 bei 2500 bar, dies entspricht $(\Delta f / \Delta p) \cdot 1000$ bar $=-0.40$. Die Effektivität sinkt um den Faktor sieben schneller als $f$ von DTBP. Der Literaturwert mit $f=0.37$ bei 2000 bar ist geringfügig kleiner als die Effektivitäten, die in beiden Messungen mit 0.39 bzw. 0.41 bestimmt worden sind [2].

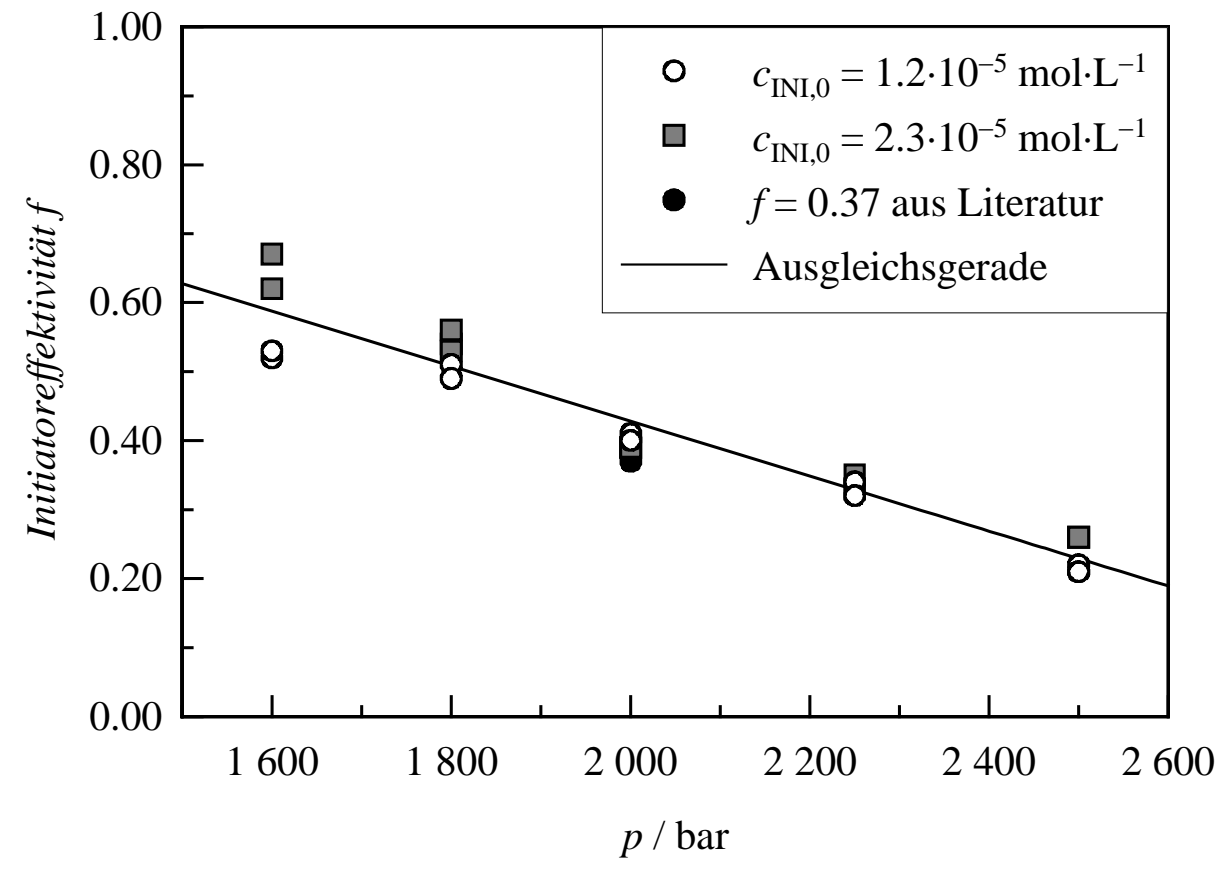

Abb. 4.44: Druckabhängigkeit der Initiatoreffektivität für TBPP. Offene Kreise $c(T B P P)_{0}=$ $1.2 \cdot 10^{-5} \mathrm{~mol} \cdot \mathrm{L}^{-1}$; gefüllte Quadrate $\mathrm{C}(\mathrm{TBPP})_{0}=2.3 \cdot 10^{-5} \mathrm{~mol} \cdot \mathrm{L}^{-1}$; $T=156$ bis $162{ }^{\circ} \mathrm{C}$ (offene Kreise), $T=163$ bis $172{ }^{\circ} \mathrm{C}$ (gefüllte Quadrate), $m_{E}=$ $706 g \cdot h^{-1}$. 


\subsubsection{Initiierungsexperimente mit tert-Butylperoxy-2-ethylhexanoat}

Bei diesem Experiment betrug die Initiatorkonzentration $c(\text { TBPO })_{0}=5.6 \cdot 10^{-6} \mathrm{~mol} \cdot \mathrm{L}^{-1}$. Die Reaktionstemperatur lag zwischen 171 und $177^{\circ} \mathrm{C}$. Der Stoffmengenanteil an Propionaldehyd betrug bei 0.0032. In Abbildung 4.45 ist die Druckabhängigkeit des TBPO-induzierten Ethenumsatzes in der Hochdruck-Polymerisation zu sehen. Wie bei den zuvor gezeigten Messungen steigt der Ethenumsatz mit dem Druck an.

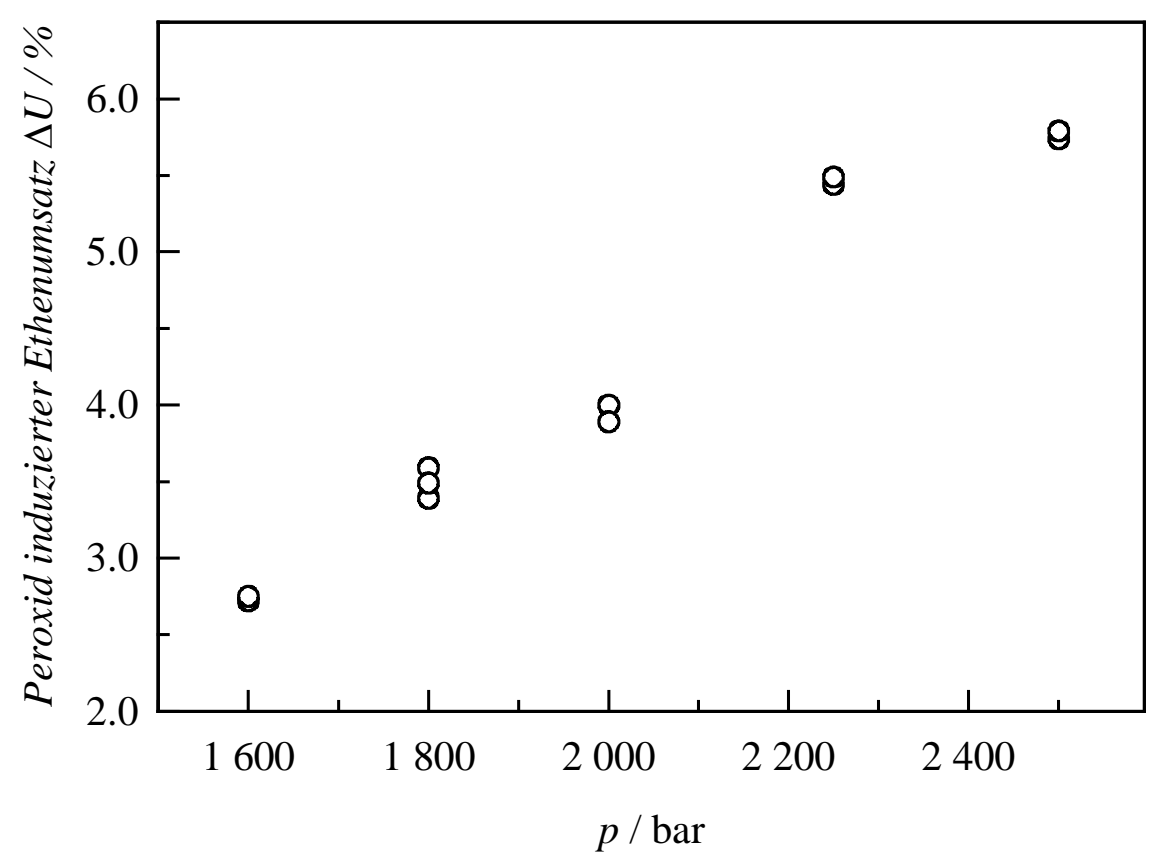

Abb. 4.45: Druckabhängigkeit des Peroxid-induzierten Ethenumsatzes für Ethen-Homopolymerisation mit TBPO als Initiator; $T=171$ bis $177^{\circ} \mathrm{C}, m_{E}=706 \mathrm{~g} \cdot \mathrm{h}^{-1} ; \mathrm{c}(\mathrm{TBPO})_{0}=5.6 \cdot 10^{-6} \mathrm{~mol} \cdot \mathrm{L}^{-1}$.

Die Initiatoreffektivität sinkt mit dem Druck (siehe Abbildung 4.46), was auch bei TBPP beobachtet wurde. Die Effektivität bei 2000 bar weicht mit 0.51 um 0.1 vom Literaturwert von $0.61 \mathrm{ab}$ [2]. Die Werte sinken von 0.67 bei 1600 bar auf 0.42 bei 2500 bar, dies entspricht $(\Delta f / \Delta p) \cdot 1000$ bar $=-0.28$. Die Steigung der Ausgleichsgerade ist geringer als bei TBPP. 


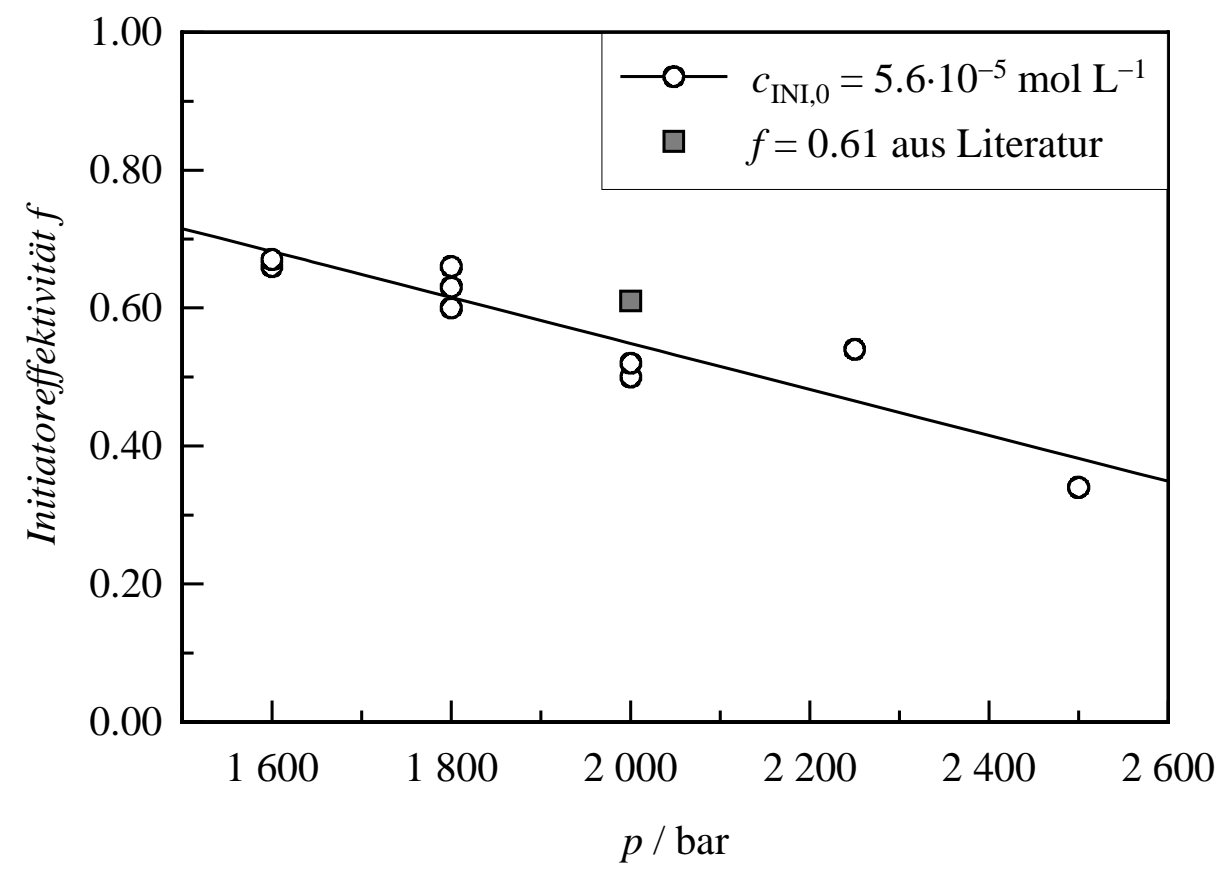

Abb. 4.46: Druckabhängigkeit der Initiatoreffektivität für TBPO.

$\mathrm{T}=171$ bis $177^{\circ} \mathrm{C}, \mathrm{m}_{\mathrm{E}}=706 \mathrm{~g} \mathrm{~h}^{-1} ; \mathrm{c}(\mathrm{TBPO})_{0}=5.6 \cdot 10^{-6} \mathrm{~mol} \mathrm{~L}$.

\subsection{Diskussion}

In diesem Abschnitt sollen die vorgestellten Ergebnisse zusammengefasst und diskutiert werden.

\subsubsection{Initiatorcocktails}

In Abschnitt 4.5 wurde die Temperaturabhängigkeit der Peroxid-induzierten Ethenumsätze von zwei Initiatorcocktails (TBPA/TBPP und BTMHP/TBPO) gezeigt. Die optimalen Temperaturen waren bei der BTMHP/TBPO-Mischung sehr ähnlich, so dass kaum Unterschiede im Umsatz-Temperatur-Profil im Vergleich zu typischen Einzelmessungen zu sehen sind. Im Fall von der TBPA/TBPP-Mischung hingegen ist der Unterschied beider optimaler Temperaturen groß. Beide optimalen Temperaturen sind im Umsatz-Temperatur-Profil zu erkennen.

Ebenfalls wurde gezeigt, dass die Initiatoreffektivitäten in Cocktails nicht getrennt von einander bestimmbar sind. Die Effektivität eines Peroxids muss bekannt sein, um $f$ des anderen Peroxids zu berechnen. Im vorliegenden Fall sind für beide Mischungen die Effektivitäten der einzelnen Peroxide bekannt, so dass es möglich war, eine Veränderung in 
der Effektivität zu beobachten. In beiden Fällen wurden Initiatoreffektivitäten erhalten, die mit den Literaturwerten übereinstimmen. Dies bedeutet, dass der schnell zerfallende Initiator (niedrigere $T_{\mathrm{opt}}$ ) den langsam zerfallenden Initiator (höhere $T_{\mathrm{opt}}$ ) bei diesen kleinen Konzentrationen $\left(10^{-6}\right.$ bis $\left.10^{-5} \mathrm{~mol} \cdot \mathrm{L}^{-1}\right)$ beim Zerfall nicht beeinflusst. Es kommt zu keinem induzierten Initiatorzerfall, der die Initiatoreffektivität herabsenkt.

\subsubsection{Asymmetrische Dialkylperoxide}

In diesem Abschnitt soll die Initiatoreffektivität der im Rahmen dieser Arbeit untersuchten asymmetrischen Dialkylperoxide mit der Initiatoreffektivität der symmetrischen Dialkylperoxide aus der Literatur verglichen werden [1, 2]. Ebenfalls wird versucht, die erhaltenden Effektivitäten zu erklären.

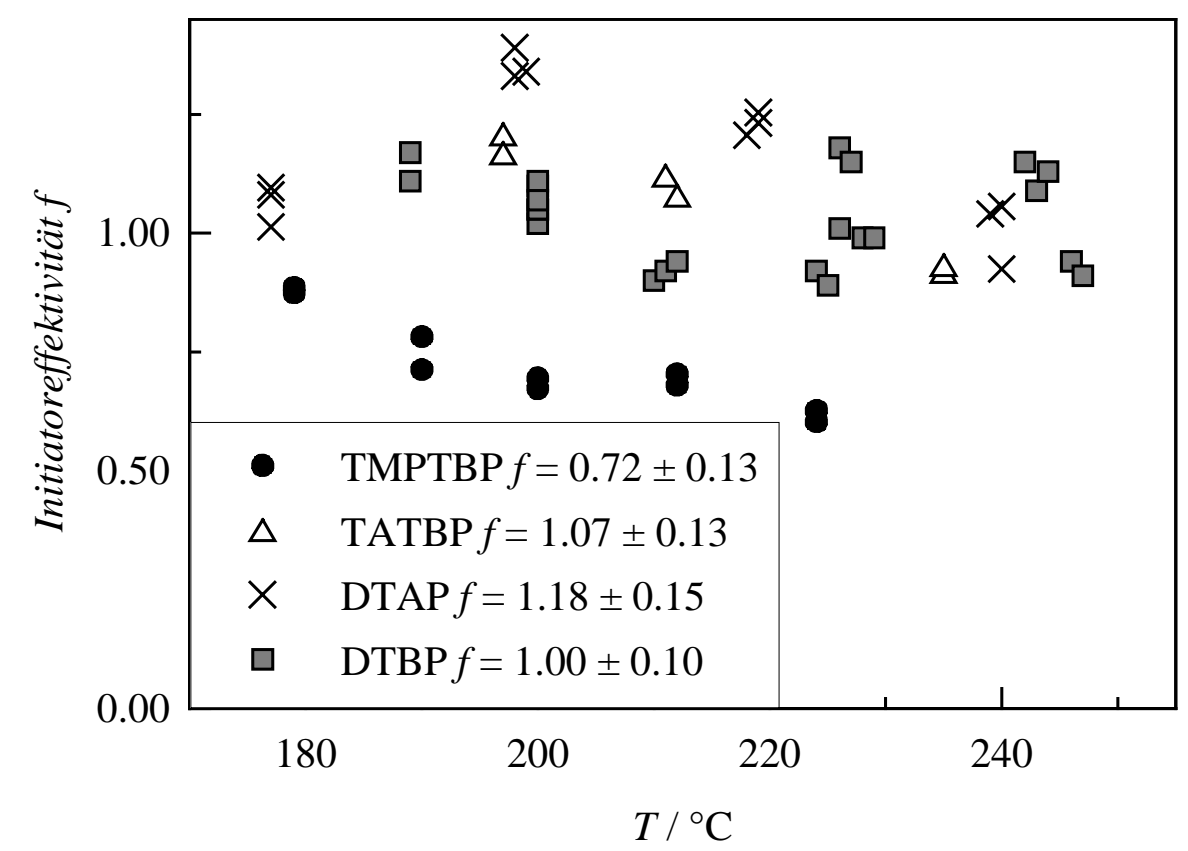

Abb. 4.47: Temperaturabhängigkeit der Initiatoreffektivitäten für TMPTBP und TATBP. Im Vergleich dazu die Temperaturabhängigkeit der korrigierten Initiatoreffektivitäten von DTBP und DTAP, aus der Literatur entnommen [1, 2]. $p=2000 \mathrm{bar}, m_{E}=706 \mathrm{~g} \cdot \mathrm{h}^{-1}$; es wurden nur Werte für $T \leq T_{\text {opt }}$ berücksichtigt.

In Abbildung 4.47 ist die Temperaturabhängigkeit der Initiatoreffektivität der im Rahmen dieser Arbeit untersuchten Dialkylperoxide TATBP und TMPTBP, sowie der korrigierten Initiatoreffektivität von Di-tert-butylperoxid (DTBP) [1, 2] und Di-tert-amylperoxid (DTAP) aus der Literatur [1] dargestellt. Bis auf TMPTBP haben alle Dialkylperoxide eine Initiator- 
effektivität um 1. Die Effektivität von TMPTBP ist mit 0.72 deutlich kleiner als die anderen Di-tert-Alkylperoxide.

Um diese Herabsetzung der Initiatoreffektivität zu erklären, müssen die strukturellen Unterschiede der Di-tert-Alkylperoxide betrachtet werden. Sie sind verantwortlich für die Geschwindigkeit der Folgereaktion nach dem primären Bindungsbruch. In Abbildung 4.48 ist ein Reaktionsschema für die thermische Zersetzung eines Dialkylperoxides gezeigt.

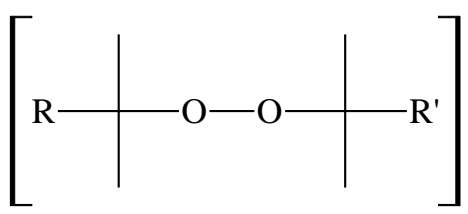

$\begin{aligned} & \text { Rekombi- } \\ & \text { nation }\end{aligned}$ $\begin{aligned} & \text { primärer } \\ & \text { Bindungsbruch }\end{aligned}$

${ }^{\mathrm{O}}+\mathrm{R} \cdot \underset{\beta \text {-scission }}{\stackrel{\text { Diffusion }}{\rightleftarrows}}\left[\mathrm{R} \longrightarrow \mathrm{O} \cdot+\cdot \mathrm{O} \longrightarrow \mathrm{R}^{\prime}\right] \frac{\text { Diffusion }}{\underset{\beta \text {-scission }}{\longrightarrow}} \cdot \mathrm{R}^{\prime}+$

$\downarrow \beta$-scission

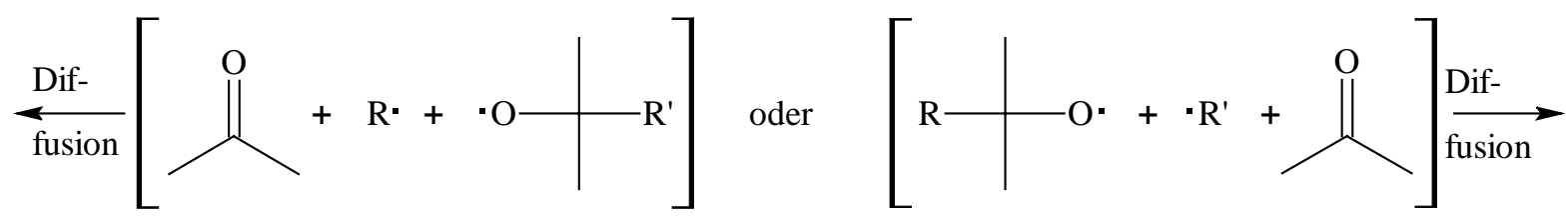

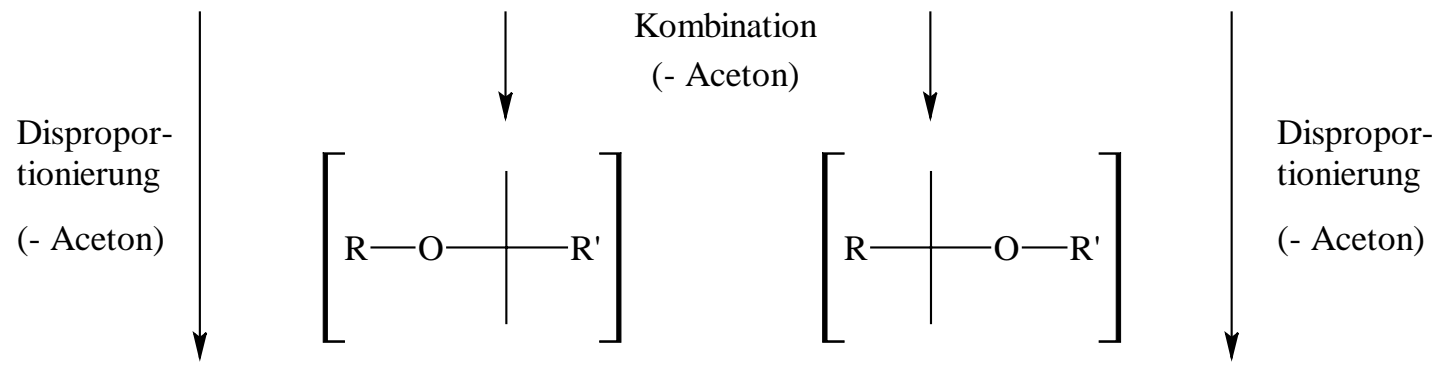

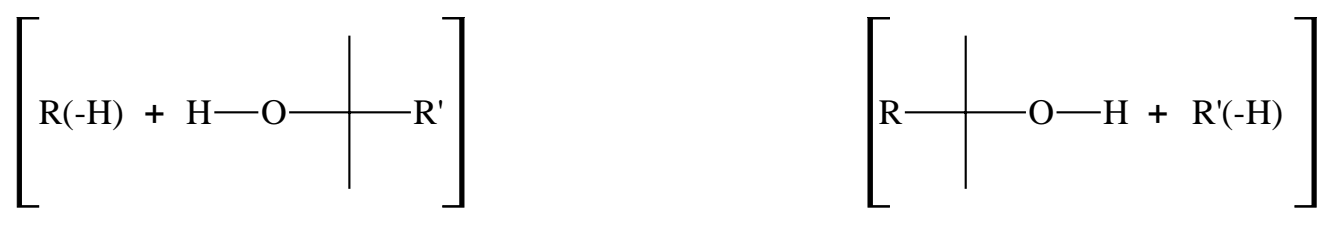

Abb. 4.48: Zerfallsschema der Di-tert-Alkylperoxide. Die Klammern symbolisieren den Lösungsmittelkäfig.

Die Klammern sollen den Lösungsmittelkäfig symbolisieren. Dieser wurde in der Literatur eingeführt, um Rekombinationsreaktionen in Lösung besser zu erklären. Es wurde festgestellt, 
dass die Rückreaktion in Lösung, im Unterschied zu Gasen, stärker berücksichtigt werden muss. Nach dem primären Bindungsbruch sind die Radikale nahe beieinander, umgeben von Molekülen des Lösungsmittels. Dies wird der Lösungsmittelkäfig genannt. Dieser Käfig verhindert, nachdem das Peroxidmolekül zerfallen ist, dass die entstandenen Radikale sich sofort voneinander trennen können. Folgereaktionen der nicht getrennten Radikale sind nun möglich.. Das kann zum einen die Rekombination zum Ausgangsmolekül oder zum anderen die $\beta$-scission der tert-Alkoxyradikale sein. Die $\beta$-scission kann auch außerhalb des Käfigs geschehen, wenn die Diffusion schneller erfolgt. Bei der $\beta$-scission wird ein Alkylradikal unter Bildung eines Ketons abgespalten. Erst durch Diffusion eines Lösungsmittelmoleküls zwischen die beiden Radikale, bzw. durch Diffusion eines der beiden Radikale aus diesem Lösungsmittelkäfig, werden die Radikale getrennt. Es hat sich gezeigt, dass mit diesem Ansatz qualitativ die Unterschiede der Initiatoreffektivität der tert-Butylperoxyester erklärt werden können [3]. Ist eine Folgereaktion schneller oder in der selben Größenordnung wie der Diffusionsschritt, so kann die Effektivität durch eine Radikalverlustreaktion als weitere Folgereaktion herabgesetzt werden. Radikalverlustreaktionen sind Kombination und Disproportionierung (H-Abstraktion). Bei beiden Reaktionen werden aus den im Lösungsmittelkäfig gefangenen Radikalen stabile Moleküle gebildet, die keine Polymerkette starten können. Die Reaktionsgeschwindigkeit der $\beta$-scission ist abhängig von der Stabilität des gebildeten Alkylradikals. In der Reihe Methyl- < primäres < sekundäres < tertiäres Radikal steigt die Stabilität der Alkylradikale an und somit die Reaktionsgeschwindigkeit der $\beta$-scission, ausgehend von den korrespondierenden Alkoxyradikalen. Das tert-Butoxyradikal kann nur in ein Methylradikal und Aceton zerfallen. Das tert-Amyloxyradikal kann in ein Methylradikal und in 2-Butanon oder in ein Ethylradikal (primäres Radikal) und Aceton zerfallen. Das 1,1,2,2-Tetramethylpropyloxyradikal kann in ein Methylradikal und ein 3,3Dimethylbutan-2-on oder in ein tert-Butylradikal (tertiäres Radikal) und Aceton zerfallen. Die $\beta$-scission des 1,1,2,2-Tetramethylpropyloxyradikals zum tert-Butylradikal ist die schnellste unter den untersuchten tert-Alkoxyradikalen. Die Geschwindigkeitskonstanten für die $\beta$-scission der hier besprochenen tert-Alkoxyradikale und für das 1,1,3,3-Tetramethylbutoxyradikal wurde von KLING und SCHMATZ durch DFT-Berechnungen erhalten [22]. 


\begin{tabular}{|c|c|c|c|}
\hline Alkoxyradikal & $k_{\beta}(1$ bar $) / s^{-1}$ bei $200{ }^{\circ} \mathrm{C}$ & $A / \mathrm{s}^{-1}$ & $E_{\mathrm{A}} / \mathbf{k J} \cdot \mathbf{m o l}^{-1}$ \\
\hline tert-Butoxy & $1.60 \cdot 10^{7}$ & $1.3 \cdot 10^{14}$ & 62.6 \\
\hline tert-Amyloxy & $9.91 \cdot 10^{8}$ & $1.1 \cdot 10^{14}$ & 45.7 \\
\hline 1,1,3,3-Tetramethylbutyloxy & $5.40 \cdot 10^{9}$ & $4.6 \cdot 10^{13}$ & 35.6 \\
\hline 1,1,2,2-Tetramethylpropyloxy & $5.56 \cdot 10^{11}$ & $1.1 \cdot 10^{14}$ & 20.8 \\
\hline
\end{tabular}

Tab. 4.4: Auf DFT-Rechnungen (UB3LYP/6-31G(d,p)) basierende Geschwindigkeitskonstanten und Aktivierungsparameter der $\beta$-scission für die untersuchten tertAlkoxyradikale [22]. Es ist jeweils die Geschwindigkeitskonstante für die schnellste Reaktion angegeben, die zum Aceton führt.

In Tabelle 4.4 sind für die $\beta$-scission-Reaktionen die berechneten Geschwindigkeitskonstanten für $200^{\circ} \mathrm{C}$ und 1 bar angegeben, bei denen Aceton und das entsprechende Radikal gebildet werden. Es ist die oben genannte Reihung der Geschwindigkeitskonstanten zu sehen. Um die Lebensdauer des Lösungsmittelkäfigs abzuschätzen, wurde die Geschwindigkeit berechnet, mit der ein Lösungsmittelmolekül einen Platzwechsel vollzieht. Dieser Geschwindigkeit kann eine Geschwindigkeitskonstante zugeordnet werden, die im nachfolgenden Text Diffusionsgeschwindigkeitskonstante $k_{\text {diff }}$ genannt wird. Die berechnete Diffusionsgeschwindigkeitskonstante $k_{\text {diff }}$ für $n$-Heptan als Lösungsmittel ist bei $200{ }^{\circ} \mathrm{C}$ und 1 bar $8.1 \cdot 10^{10} \mathrm{~s}^{-1}$. Dieser Wert wurde aus Dichtedaten von $n$-Heptan berechnet (siehe Kapitel 5). n-Heptan gilt als gute Modellsubstanz für die Ethen/Polymer-Lösung, da zum einen n-Heptan als Ersatz für die Polymerkette gesehen werden kann und zum anderen die Dichten von $n$-Heptan und der Ethen/Polyethylen-Lösung bei 2000 bar ähnlich sind. Die Geschwindigkeitskonstanten der $\beta$-scission bzw. der Diffusion, deuten darauf hin, dass das tert-Butoxyradikal den Käfig unbeschadet verlässt und erst außerhalb des Käfigs zerfällt. Das 1,1,2,2-Tetramethylpropyloxyradikal zerfällt innerhalb des Käfigs. Für das tert-Amyloxy- und 1,1,3,3-Tetramethylbutoxyradikal ist es nicht eindeutig, ob die $\beta$-scission inner- oder außerhalb des Käfigs erfolgt. Bei der Hochdruckpolymerisation von Ethen wurde für TATBP eine Effektivität von eins erhalten. Im Umkehrschluss bedeutet dies, dass die $\beta$-scission des tert-Amyloxy- und tert-Butoxyradikals bei diesen Temperaturen außerhalb des Käfigs erfolgt. Die $\beta$-scission des 1,1,2,2-Tetramethylpropyloxyradikals hingegen findet im Käfig statt. Somit können innerhalb der Käfiglebensdauer das tert-Butyl- und tert-Butoxyradikal miteinander reagieren. Dies erklärt die für Dialkylperoxide relativ niedrige Effektivität von 0.72 . 


\subsubsection{Peroxyester}

In diesem Abschnitt werden die Initiatoreffektivitäten der Peroxyester, die in Abschnitt 4.7, 4.8 und 4.9 vorgestellt worden sind, diskutiert und verglichen.

In Abbildung 4.49 ist die Temperaturabhängigkeit der Effektivität der tert-Amylperoxyester zu sehen. Die primären tert-Amylperoxyester TAPA und TAPnB zeigen mit $f$ von 0.69 bzw. 0.73 die höchste Initiatoreffektivität der fünf untersuchten tert-Amylperoxyestern. Die sekundären tert-Amylperoxyester TAPiB und TAPO besitzen eine Effektivität von 0.56 bzw. 0.61 und sind damit 0.13 unterhalb der primären tert-Amylperoxyester. Für das tertiäre tert-Amylperoxypivalat TAPP wird eine Initiatoreffektivität von 0.48 erhalten. Das ist eine Herabsetzung der Initiatioreffektivität von 0.1 gegenüber dem sekundären tertAmylperoxyester.

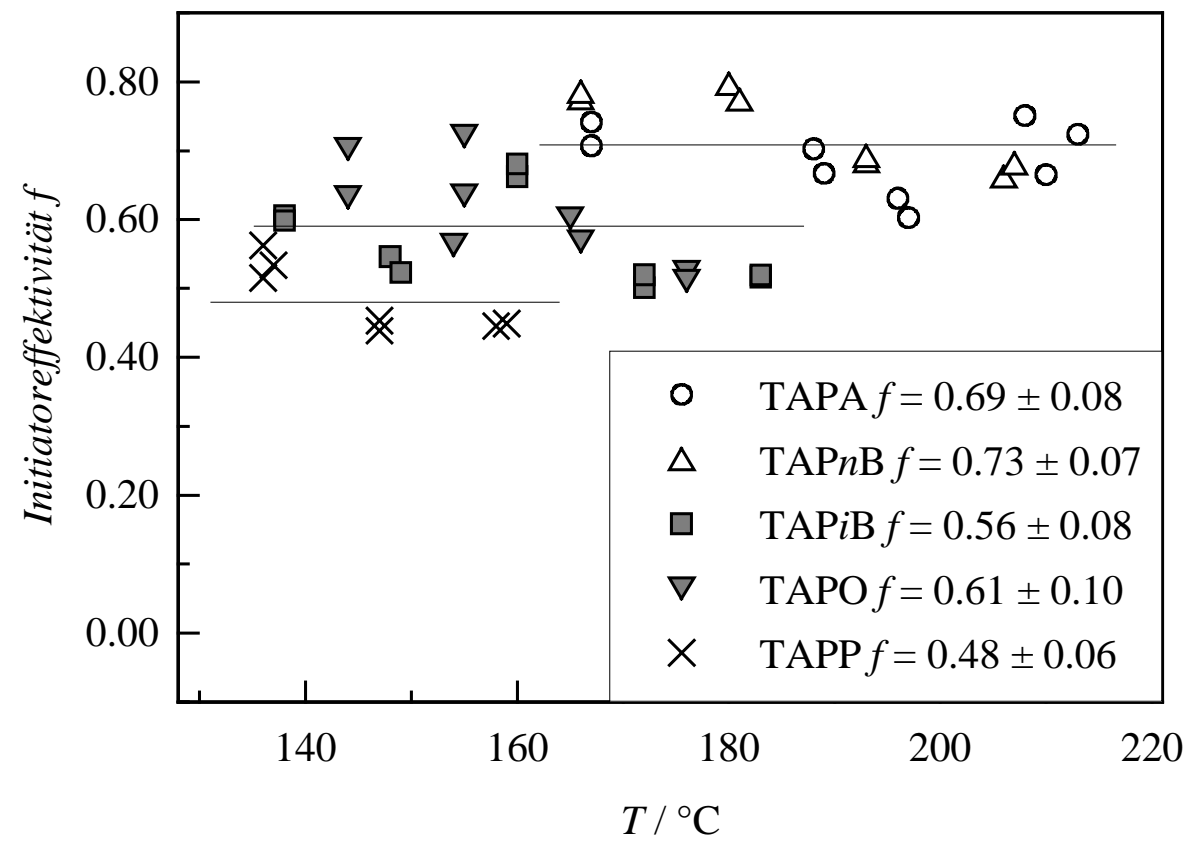

Abb. 4.49: Temperaturabhängigkeit der Initiatoreffektivität für die untersuchten tert-Amylperoxyester.

$p=2000 \mathrm{bar}, m_{E}=706 \mathrm{~g} \cdot \mathrm{h}^{-1}$; es wurden nur Werte für $T \leq T_{\text {opt }}$ berücksichtigt.

Für mehrere 1,1,3,3-Tetramethylbutylperoxyester ist die Temperaturabhängigkeit der Initiatoreffektivität in Abbildung 4.50 gezeigt. 


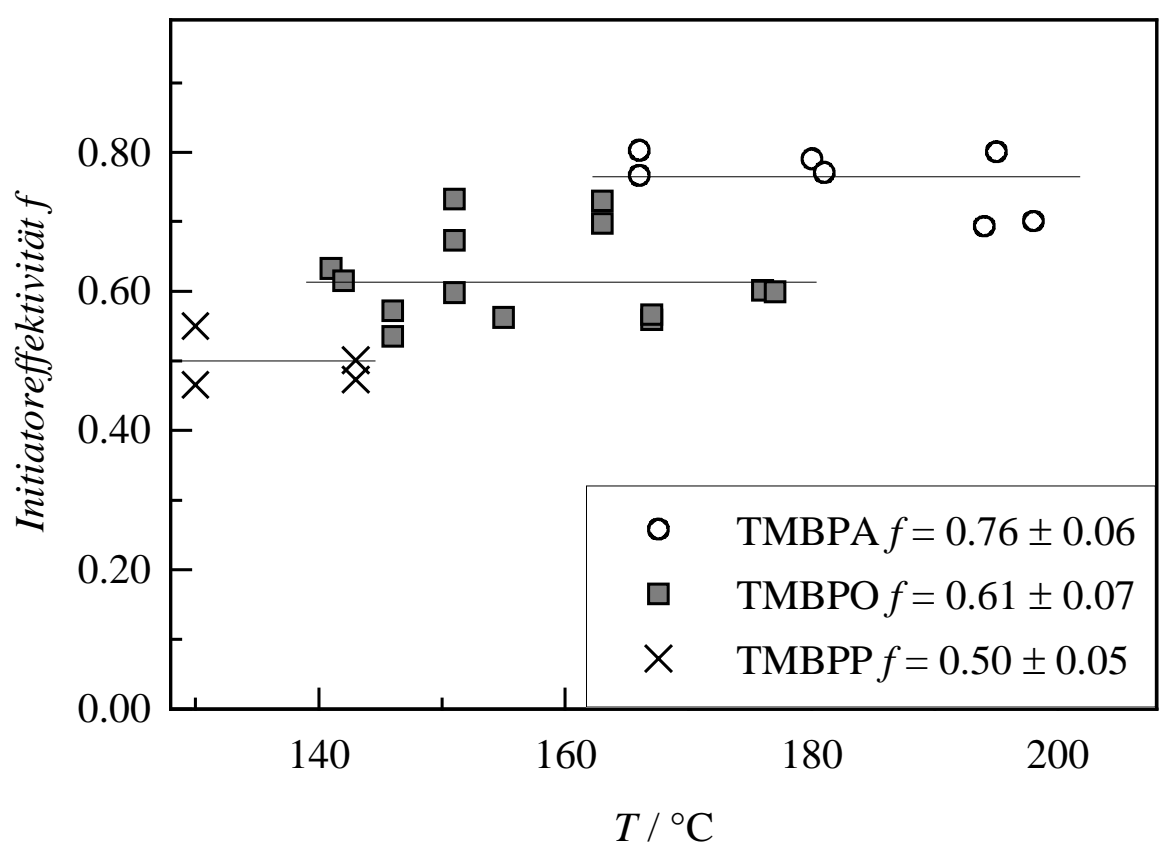

Abb. 4.50: Temperaturabhängigkeit der Initiatoreffektivität für die untersuchten 1,1,3,3-Tetramethylbutylperoxyester.

$p=2000 \mathrm{bar}, m_{E}=706 \mathrm{~g} \cdot \mathrm{h}^{-1}$; es wurden nur Werte für $T \leq T_{\text {opt }}$ berücksichtigt.

TMBPA hat eine Initiatoreffektivität von 0.76, TMBPO von 0.61 und TMBPP von 0.50. Die ähnlichen Initiatoreffektivitäten von den 1,1,3,3-Tetramethylbutyl- und tert-Amylperoxyester mit gleicher Säureseite lassen sich dadurch erklären, dass die $\beta$-scission des 1,1,3,3-Tetramethylbutyloxyradikals ähnlich schnell ist, wie die des tert-Amyloxyradikals, da bei beiden Radikalen bei der $\beta$-scission-Reaktion bevorzugt ein primäres Radikal (Ethylradikal beim tert-Amyloxyradikal, bzw. neo-Pentylradiakl beim 1,1,3,3-Tetramethylbutyloxyradikal) abgespalten wird. Die $\beta$-scission zum Methylradikal ist im Vergleich zur $\beta$-scission zu dem primären Radikal langsam. In Tabelle 4.4 im Kapitel 4.11.2 sind Geschwindigkeitskonstanten der $\beta$-scission für die tert-Alkoxyradikale aufgelistet, die beim Zerfall der im Rahmen dieser Arbeit untersuchten Initiatoren auftreten.

Aufgrund der $\beta$-scission innerhalb des Käfigs, die zur Radikalverlustreaktionen führt, ist die Effektivität der 1,1,2,2-Tetramethylpropylperoxyestern sehr niedrig. In Abbildung 4.51 ist die Temperaturabhängigkeit der Initiatoreffektivität für TMPPA und TMPPP dargestellt. Für beide Initiatoren ist $f=0.50$. Die Daten für TMPPA sind aus der Literatur entnommen [3]. 


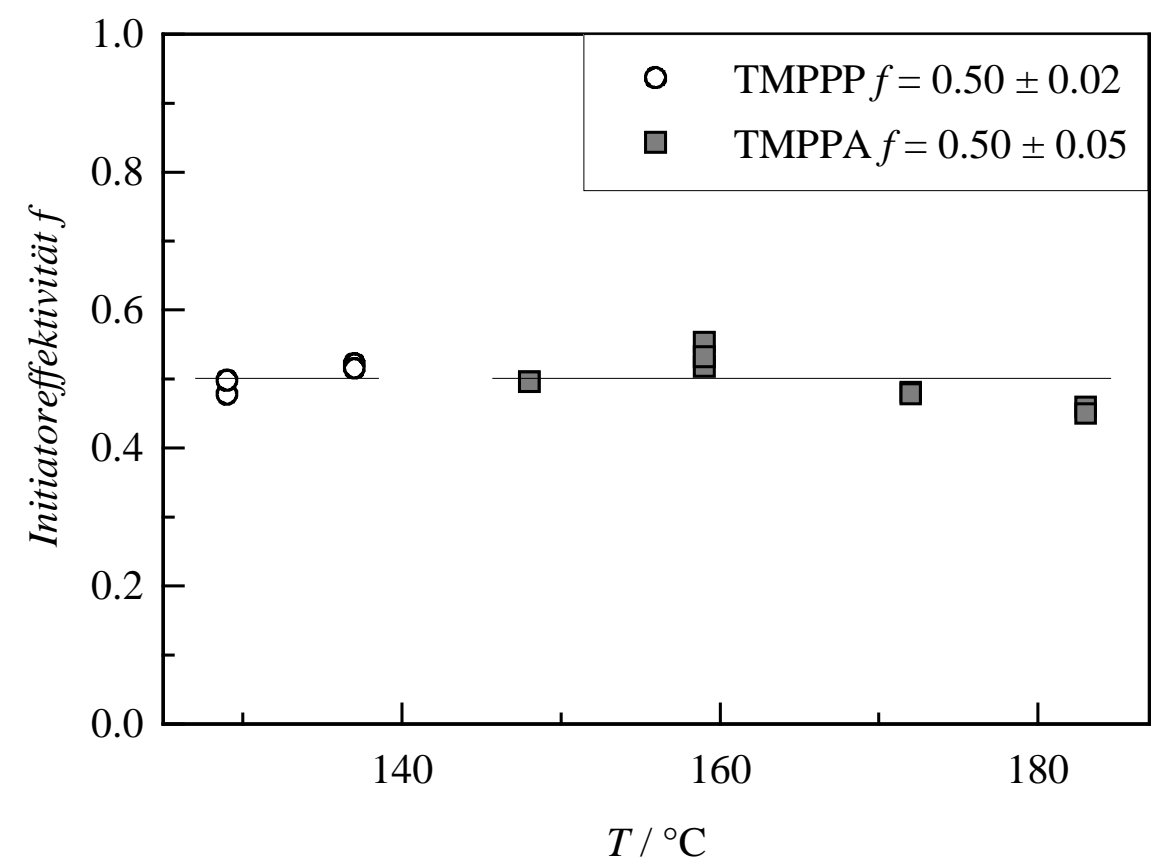

Abb. 4.51: Temperaturabhängigkeit der Initiatoreffektivität für das untersuchte 1,1,2,2-Tetramethylpropylperoxypivalat. Im Vergleich dazu $f$ von TMPPA aus der Literatur entnommen [1].

$p=2000 \mathrm{bar}, m_{E}=706 \mathrm{~g} \cdot \mathrm{h}^{-1}$; es wurden nur Werte für $T \leq T_{\text {opt }}$ berücksichtigt.

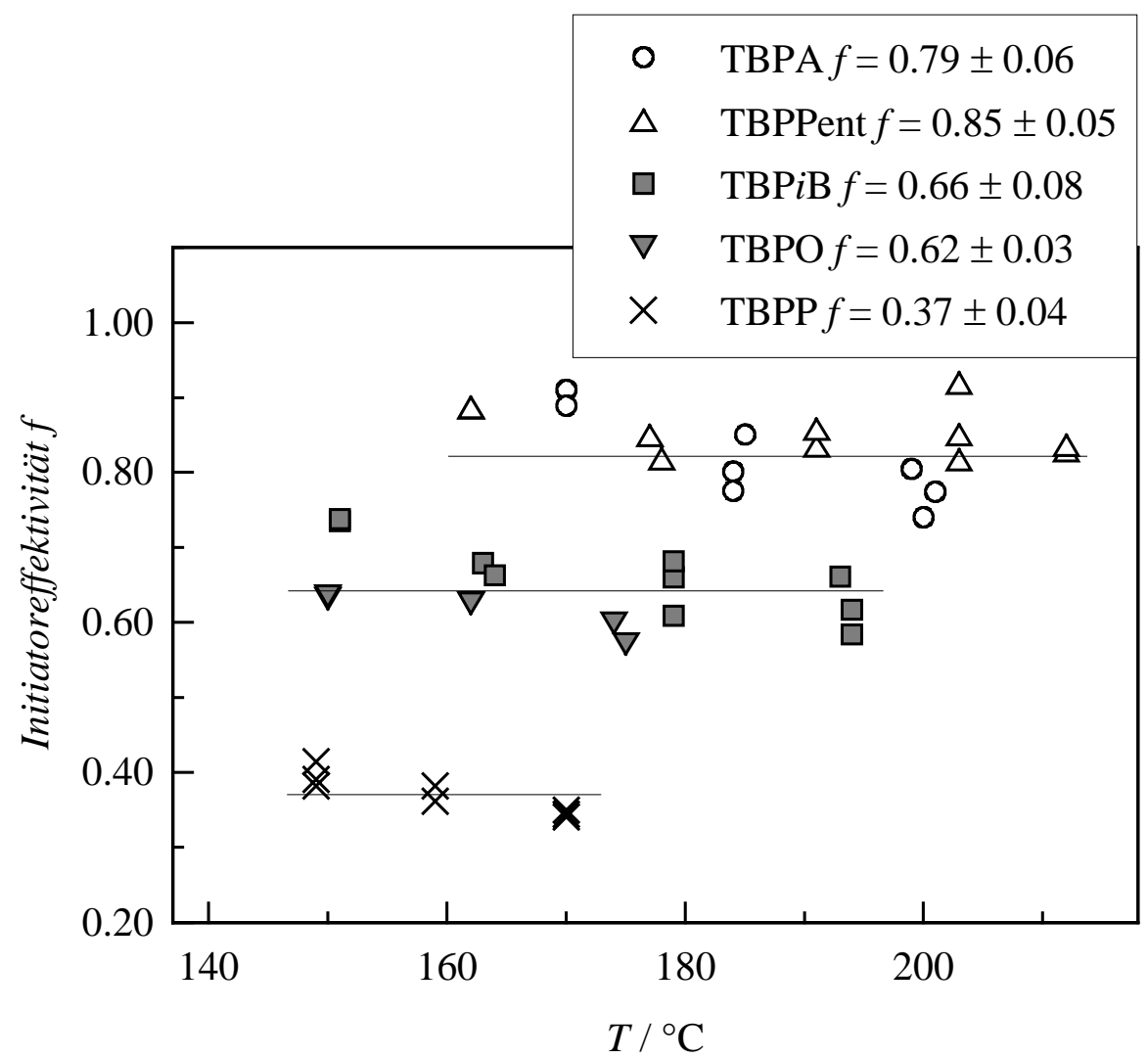

Abb. 4.52: Korrigierte Literaturwerte für die Temperaturabhängigkeit der Initiatoreffektivität der tert-Butylperoxyester [2, 3, 16].

$p=2000$ bar, $m_{E}=706 \mathrm{~g} \cdot \mathrm{h}^{-1}$; es wurden nur Werte für $T \leq T_{\text {opt }}$ berücksichtigt. 
In vorangegangenen Arbeiten wurden Experimente mit der selben Apparatur mit tert-Butylperoxyestern als Initiatoren in der Ethen-Hochdruckpolymerisation durchgeführt [2, 3, 16]. Die erhaltenen Initiatoreffektivitäten sind in Abbildung 4.52 in Abhängigkeit von der Temperatur dargestellt.

Bei den tert-Butylperoxyestern ist der Effektivitätsunterschied bei Variation der Säureseite am stärksten. Die Initiatoreffektivität sinkt von den primären Peroxyestern mit 0.85 für TBPPent bzw. 0.79 für TBPA über die sekundären Peroxyestern mit 0.66 für TBPiB bzw. 0.62 für TBPO auf 0.37 des tertiären TBPP.

In Abbildung 4.53 sind die Initiatoreffektivitäten der tert-Alkylperoxyester aus der Hochdruckpolymerisation von Ethen zusammengefasst [2, 3, 16]. Es wurde die Alkoholseite, sowie die Säureseite verändert. Für die primären (TBPA und TBPPent bzw. TAPA und TAPnB) und sekundären (TBPiB und TBPO bzw. TAPiB und TAPO) tert-Butyl- und tertAmylperoxyester wurde jeweils ein Mittelwert gebildet.

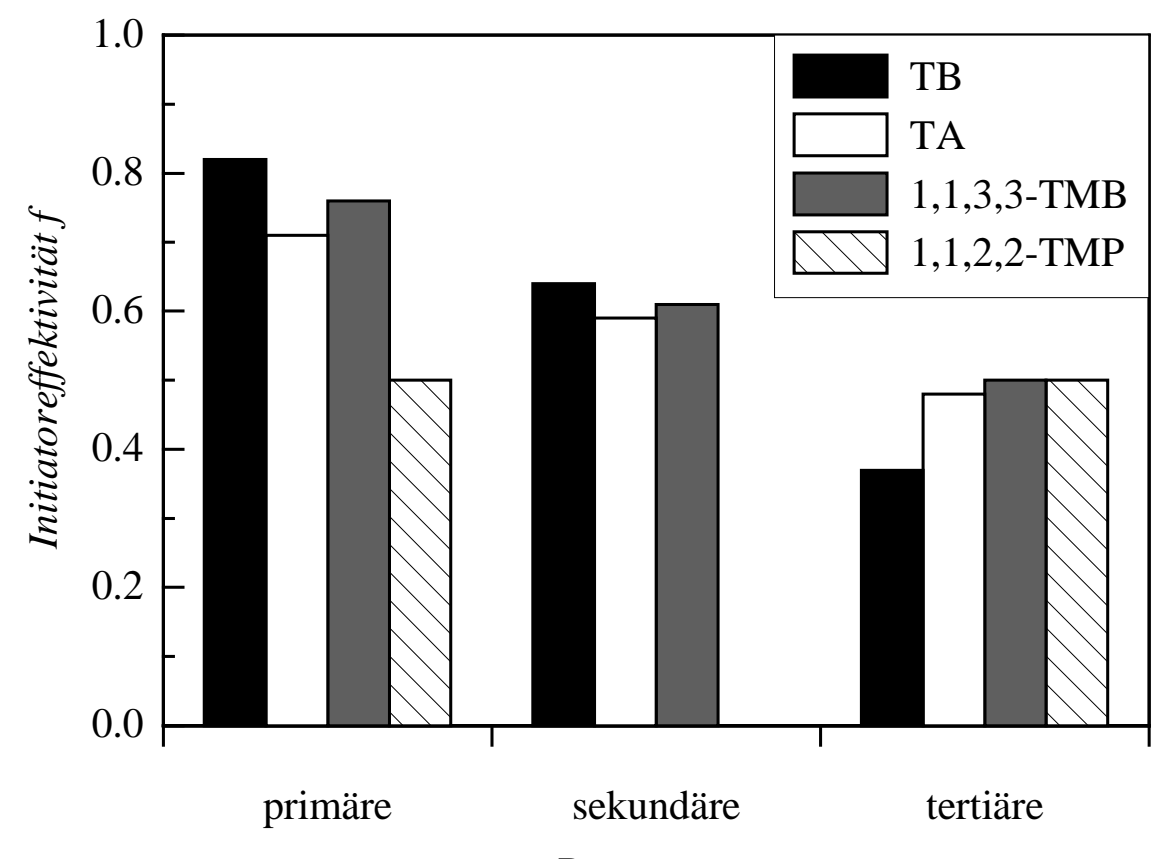

Peroxyester

Abb. 4.53: Abhängigkeit der Initiatoreffektivität der untersuchten tert-Alkylperoxyester vom Säurerest. Die Literaturwerte der tert-Butylperoxyester und der Literaturwert von TMPPA sind zum Vergleich angegeben [2, 3, 16].

Die nachfolgenden Beobachtungen können gemacht werden:

(a) Die Effektivität der tert-Butyl-, tert-Amyl- und 1,1,3,3-Tetramethylbutylperoxyester sinkt vom primären zum tertiären Peroxyester. 
(b) Die Effektivität der tert-Amyl- und 1,1,3,3-Tetramethylbutylperoxyester (mit der gleichen Säureseite) ist nahezu identisch.

(c) Die Effektivität der tert-Butylperoxyester ist verglichen zu den 1,1,2,2-Tetramethylpropylperoxyester für die primären höher, aber niedriger für die tertiären Peroxyester.

(d) Die Effektivität der primären und tertiären 1,1,2,2-Tetramethylpropylperoxyester ist identisch.

All diese Effekte können auf der Basis der unterschiedlichen Zeitskalen der Folgereaktionen (Decarboxylierung der Acyloxyradikale, bzw. $\beta$-scission der Alkoxyradikale) nach dem primären Bindungsbruch und der Reaktivität der entstehenden Radikale erklärt werden. Es ist besonders wichtig, ob die Folgereaktionen innerhalb des Käfigs erfolgen, oder nicht. Zusätzlich spielt die Art des Radikals eine wichtige Rolle. So kann die Disproportionierungsreaktion innerhalb des Lösungsmittelkäfigs im Falle eines tertiären kohlenstoffzentrierten Radikals mit einem sauerstoffzentrierten Radikal besonders schnell erfolgen, wie die Kombination von primären Alkylradikalen (siehe Kapitel 6).

Effekt (a) ist leicht zu verstehen, da dieser von der Decarboxylierung herrührt, die schneller wird beim Übergang vom primären zum sekundären und tertiären Peroxyester. Die Decarboxylierung der primären Peroxyester erfolgt hauptsächlich außerhalb des Käfigs, hingegen die der sekundären und tertiären Peroxyester innerhalb des Käfigs ( $f$ (TBPA) $=0.79$, $f($ TBPO $)=0.62$ und $f($ TBPP $)=0.37)$.

Effekt (b) wurde bereits durch die ähnlichen Geschwindigkeitskonstanten der $\beta$-scission für die zwei tert-Alkoxyradikale erklärt $(f($ TAPA, TMBPA $) \approx 0.7, f($ TAPO, TMBPO) $\approx 0.6$ und $f($ TAPP, TMBPP $) \approx 0.5)$.

Effekt (c) kann durch die schnelle Disproportionierung des Acetoxyradikals mit dem tert-Butylradikal erklärt werden, weil die $\beta$-scission des 1,1,2,2-Tertametylpropyloxyradikals schneller ist als die Decarboxylierung des Acetoxyradikals. Bei TBPA kommt es nur zur langsameren Kombination des tert-Butoxyradikals mit dem Methylradikal (Decarboxylierung des Acetoxyradikals) $(f($ TBPA $)=0.79, f($ TMPPA $)=0.50)$.

Bei TMPPP werden schnell durch $\beta$-scission und Decarboxylierung zwei tert-Butylradikale gebildet, die nicht so schnell miteinander reagieren, wie das tert-Butoxy- und tert-Butylradikal durch Disproportionierung, so dass die Initiatoreffektivität von TBPP $(f=0.37)$ kleiner ist als die von TMPPP $(f=0.48)$.

Effekt (d) ist damit zu erklären, dass bei TMPPA die $\beta$-scission schneller erfolgt als die Decarboxylierung, so dass das Acetoxyradikal mit dem tert-Butylradikal reagieren kann ( $f=$ 
0.50). Bei TMPPP ist es umgekehrt. Die Decarboxylierung ist schneller als die $\beta$-scission, so dass es zwischen dem tert-Butylradikal und dem 1,1,2,2-Tetramethylpropyloxyradikal zur Reaktion kommen kann $(f=0.50)$.

In Tabelle 3 sind die Initiatoreffektivitäten der tert-Alkylperoxyester zusammengefasst.

\begin{tabular}{ccc}
\hline Peroxyester & $\boldsymbol{f}$ & $\boldsymbol{f}_{\max }-\boldsymbol{f}_{\min }$ \\
\hline TBPA [16] & $0.79 \pm 0.06$ & $0.74-0.85$ \\
TBPPent [3] & $0.85 \pm 0.05$ & $0.82-0.92$ \\
TBPiB [16] & $0.66 \pm 0.08$ & $0.58-0.74$ \\
TBPO [2, 3] & $0.62 \pm 0.03$ & $0.57-0.63$ \\
TBPP [2, 3] & $0.37 \pm 0.04$ & $0.34-0.41$ \\
\hline TAPA & $0.69 \pm 0.08$ & $0.60-0.75$ \\
TAPnB & $0.73 \pm 0.07$ & $0.66-0.79$ \\
TAPiB & $0.56 \pm 0.08$ & $0.52-0.68$ \\
TAPO & $0.61 \pm 0.10$ & $0.52-0.72$ \\
TAPP & $0.48 \pm 0.06$ & $0.44-0.56$ \\
\hline TMBPA & $0.76 \pm 0.06$ & $0.69-0.80$ \\
TMBPO & $0.61 \pm 0.07$ & $0.60-0.73$ \\
TMBPP & $0.50 \pm 0.05$ & $0.46-0.55$ \\
\hline TMPPA [3] & $0.50 \pm 0.05$ & $0.45-0.55$ \\
TMPPP & $0.50 \pm 0.02$ & $0.48-0.52$ \\
\hline
\end{tabular}

Tab.4.5: $\quad$ Übersicht der Initiatoreffektivitäten für die im Rahmen dieser Arbeit untersuchten tert-Alkylperoxyester und zum Vergleich die Literaturwerte für die tert-Butylperoxyester bzw. für TMPPA [2, 3, 16].

\subsubsection{Druckabhängigkeit der Initiatoreffektivität}

In Abschnitt 4.7 wurde gezeigt, dass die im Rahmen dieser Arbeit untersuchten Peroxide DTBP, TBPO und TBPP eine Druckabhängigkeit in der Initiatoreffektivität besitzen. Diese Druckabhängigkeit ist unterschiedlich stark ausgeprägt. Die Initiatoreffektivität von TBPP zeigt die stärkste Druckabhängigkeit. DTBP hingegen besitzt nur eine schwache Druckabhängigkeit bezüglich der Initiatoreffektivität. Die Druckabhängigkeit der Initiatoreffektivität von TBPO ist weniger stark ausgeprägt als bei TBPP, aber stärker als bei DTBP. 


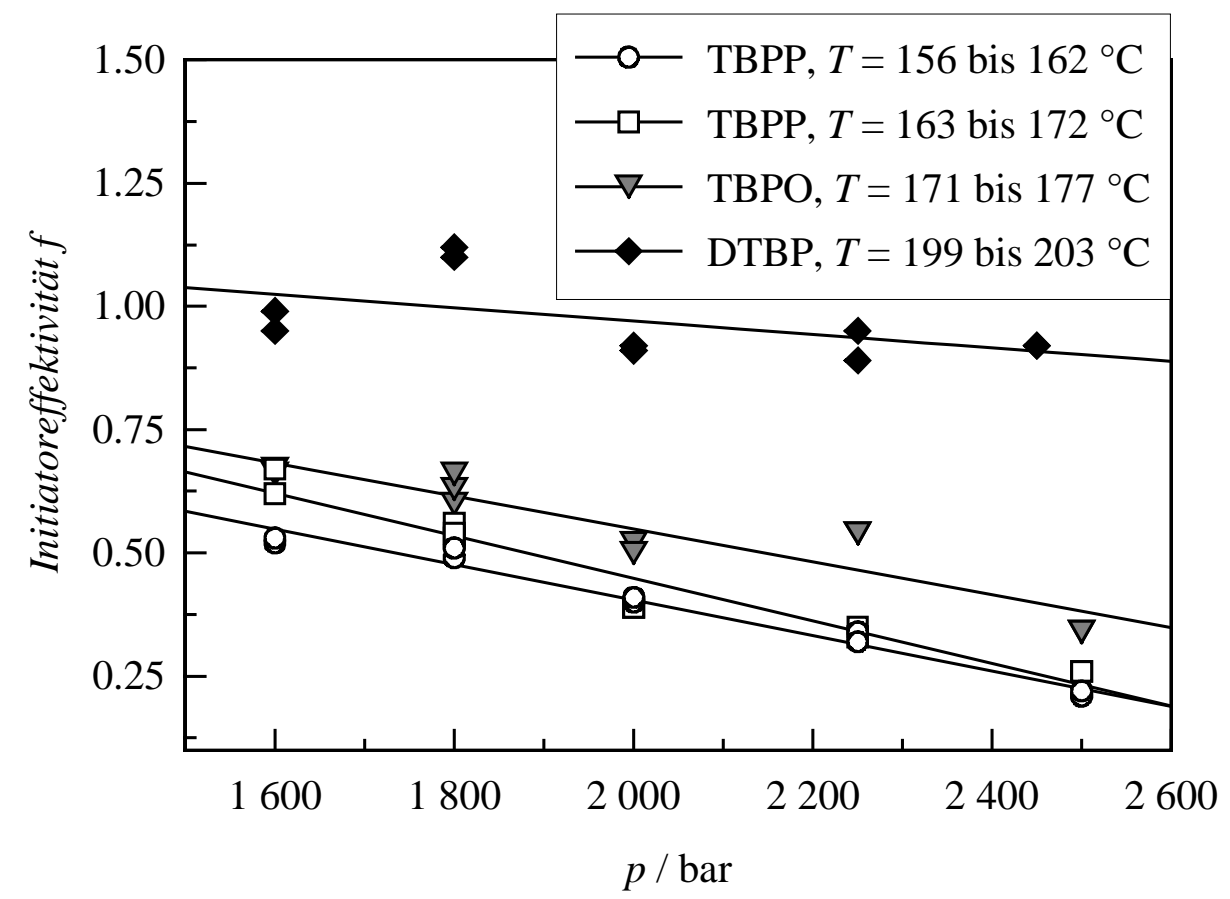

Abb. 4.54: Druckabhängigkeit von $f$ der untersuchten Peroxide. Die offenen Symbole stellen die Experimente mit TBPP dar, die grauen Symbole das Experiment mit TBPO und die schwarzen Symbole das Experiment mit DTBP.

In Abbildung 4.54 ist die Druckabhängigkeit der Initiatoreffektivität für die vier Experimente gezeigt. Die als Maß der Druckabhängigkeit angegebene Änderung von $f$ für einen Druckbereich von 1000 bar $(\Delta f / \Delta p) \cdot 1000$ bar ist in Tabelle 4.6 aufgelistet.

\begin{tabular}{ccc}
\hline Peroxid & $(\boldsymbol{\Delta} \boldsymbol{f} / \boldsymbol{\Delta} \boldsymbol{p}) \cdot \mathbf{1 0 0 0} \mathbf{b a r}$ & $\Delta \boldsymbol{V}^{\ddagger}\left(\boldsymbol{k}_{\mathbf{o b s}}\right) / \mathbf{c m}^{\mathbf{3}} \cdot \mathbf{m o l}^{-\mathbf{1}}$ \\
\hline DTBP & 0.06 & $10.1[18]$ \\
TBPO & 0.28 & $3.6[13]$ \\
TBPP & 0.40 & $3.0[12]$ \\
\hline
\end{tabular}

Tab. 4.6: Druckabhängigkeit der Initiatoreffektivität verglichen mit dem Aktivierungsvolumen des Geschwindigkeitskoeffizienten des thermischen Zerfalls aus der Literatur [18, 12, 13].

$(\Delta f / \Delta p) \cdot 1000$ bar steigt stark an von 0.06 für DTBP auf 0.40 für TBPP. Die Aktivierungsvolumina für den Geschwindigkeitskoeffizienten des thermischen Zerfalls sind für die drei Peroxide in Tabelle 4.6 angegeben. Diese sind für TBPP 3.0, für TBPO 3.6 und für DTBP $10.1 \mathrm{~cm}^{3} \cdot \mathrm{mol}^{-1}[18,12,13]$. Die Reihenfolge von $\Delta V^{\ddagger}\left(k_{\mathrm{obs}}\right)$ verhält sich umgekehrt wie $(\Delta f / \Delta p) \cdot 1000$ bar. 
Die großen Unterschiede in $(\Delta f / \Delta p) \cdot 1000$ bar lassen sich durch den Diffusionsschritt beim thermischen Zerfall der Peroxide erklären. Bei DTBP ist dieser Diffusionsschritt im Geschwindigkeitskoeffizienten des thermischen Zerfalls bereits enthalten, weil die Abnahme der DTBP-Konzentration nur durch einen diffusiven Schritt ermöglicht wird. Andere Reaktionen ( $\beta$-scission / Decarboxylierung), die eine Rückreaktion zum DTBP verhindern, sind zu langsam oder nicht möglich. Der Diffusionsschritt bedeutet eine große räumliche Trennung der entstandenen Radikale. Dies findet sich in einem großen Aktivierungsvolumen der Geschwindigkeitskonstante des thermischen Zerfalls wieder. Da nach erfolgreichem thermischen Zerfall die Radikale schon getrennt werden, ist $(\Delta f / \Delta p) \cdot 1000$ bar klein. Für TBPO und TBPP hingegen ist die Diffusion beim thermischen Zerfall weniger wichtig, weil hier die Decarboxylierung, bei TBPO zum Teil und bei TBPP vollständig, innerhalb der Käfiglebensdauer erfolgt und eine Rückreaktion zum Ausgangsmolekül verhindert. Dies hat zur Folge, dass das Aktivierungsvolumen des Geschwindigkeitskoeffizienten des thermischen Zerfalls klein ist. Aber durch die schnelle Decarboxylierung können Käfigreaktionen (Kombination / Disproportionierung (H-Abstraktion)) ein druckabhängiges $f$ ergeben. Die Initiatoreffektivität beider Peroxyester wird durch die Diffusion bestimmt. ( $\Delta f / \Delta p) \cdot 1000$ bar ist dadurch größer als das Aktivierungsvolumen von DTBP. Für TBPO ist $(\Delta f / \Delta p) \cdot 1000$ bar nicht so ausgeprägt wie beim TBPP, weil die Decarboxylierung nicht ausreichend schnell ist.

\subsubsection{Zusammenfassung der Diskussion}

Die in Kapitel 4 diskutierten Punkte lassen sich wie folgt zusammenfassen:

Es konnte gezeigt werden, dass bei den verwendeten Initiatorkonzentrationen von $10^{-5}$ bis $10^{-6} \mathrm{~mol} \cdot \mathrm{L}^{-1}$ die Initiatoreffektivität der untersuchten Peroxide in einem Initiatorcocktail sich nicht gegenseitig beeinflussen. Die mit Gleichung 4.16 erhaltenden Effektivitäten stimmten mit den Initiatoreffektivitäten aus den Einzelmessungen überein.

Es wurde ebenfalls gezeigt, dass die Initiatoreffektivität der Dialkylperoxide und tert-Alkylperoxyester stark von der Konkurrenz der Folgereaktionen des primären Bindungsbruchs und der Diffusion abhängt. Die Geschwindigkeiten dieser Folgereaktionen sind von der Konstitution des Peroxidmoleküls abhängig. Durch Variation des Säure- und Alkoholrestes bei den Peroxyestern wurden gezeigt, dass beide Seiten zum Absenken der Effektivität beitragen können, und dass die Änderung der Säureseite $f$ stärker beeinflusst als die Änderung auf der Alkoholseite. Die gleichzeitige Anwesenheit des sauerstoffzentrierten Radikals und des tert-Butylradikals bewirkt die stärkste Reduktion der Effektivität $(f(\mathrm{TBP})=0.37)$. 
Eine Druckabhängigkeit der Initiatoreffektivität wurde erstmals für TBPP, TBPO und DTBP untersucht. In einem Druckbereich von 1600 bis 2500 bar wurde gezeigt, wie der Ethenumsatz mit dem Druck steigt, aber die Initiatoreffektivität mit dem Druck sinkt. Für die drei untersuchten Peroxide wurde als Maß der Druckabhängigkeit der Initiatoreffektivität $(\Delta f / \Delta p) \cdot 1000$ bar erhalten. $(\Delta f / \Delta p) \cdot 1000$ bar ist mit 0.40 für TBPP am größten, gefolgt von TBPO mit 0.28. DTBP besitzt das kleinste $(\Delta f / \Delta p) \cdot 1000$ bar mit 0.06. $(\Delta f / \Delta p) \cdot 1000$ bar wurde mit den Literaturwerten des Aktivierungsvolumens der Geschwindigkeitskonstante für den thermischen Zerfall $\Delta V^{\ddagger}\left(k_{\text {obs }}\right)$ verglichen. Es wurde festgestellt, dass $\Delta V^{\ddagger}\left(k_{\text {obs }}\right)$ sich entgegengesetzt zu $(\Delta f / \Delta p) \cdot 1000$ bar verhält.

Dies wurde wiederum mit der Konkurrenz der Folgereaktionen nach dem primären Bindungsbruch erklärt. 


\subsection{Literaturverzeichnis für Kapitel 4}

[1] P. Becker, Dissertation, Göttingen (2001)

[2] P. Becker, M. Buback, J. Sandmann, Macromol. Chem. Phys. 203 (2002) 2113

[3] J. Sandmann, Dissertation, Göttingen (2000)

[4] T. J. van der Molen, C. van Heerden, Advances in Chemistry Series, Ed. K. B. Bischoff, American Chemical Society, Washington (1972) 92

[5] G. Luft, H. Bitch, H. Seidl, J. Macromol. Sci. Chem. A11 (1977) 1089

[6] R. A. Hutchinson, R. E. Fuller, Dechema Monographs 134 (1998) 35

[7] J. Schweer, Dissertation, Göttingen (1988)

[8] E. Fitzer, W. Fritz, G. Emig, Technische Chemie, Springer-Verlag, Berlin (1995)

[9] M. Buback, Z. Naturforsch. 39a (1984) 399

[10] T. v. d. Molen, A. Koenen, H. Oosterwijk, H. v. d. Bend, Ing Chim. Ital. 18 (1982) 7

[11] S. Jauer, Dissertation, Göttingen (2005)

[12] M. Buback, S. Klingbeil, J. Sandmann, M.-B. Sderra, H. P. Vögele, H. Wackerbarth,

L. Wittkowski, Z. Phys. Chem., 210 (1999) 199

[13] M. Buback, J. Sandmann, Z. Phys. Chem., 214 (2000) 583

[14] C. Hinton, Dissertation, Göttingen (1995)

[15] P. Becker, persönliche Mitteilung, (2000)

[16] S. Jauer, persönliche Mitteilung, (2001)

[17] K. Matsuyama, Y. Higuchi, Bull. Chem. Soc. Jpn, 64 (1991) 259

[18] M. Buback, H. Lendle, Z. Naturforsch. 34a (1979) 1482

[19] H.-P. Vögele, unveröffentliche Ergebnisse

[20] M. Buback, D. Nelke, H. P. Vögele, Z. Phys. Chem., 217 (2003) 1169

[21] F. Becker, M. Buback, H. Latz, G. Sadowski, F. Tumakaka, Fluid Phase Equilibria, 215 (2004) 263

[22] M. Buback, M. Kling, S. Schmatz, eingereicht, 2005 


\section{Kapitel 5}

\section{Modellierung von Käfigreaktionen durch Analyse von Zerfallsprodukten in $n$-Heptan}

\subsection{Einleitung}

Es ist wünschenswert die Initiatoreffektivität von Peroxiden mittels kinetischer Modellierung vorhersagen zu können. Als Ansatz wurden folgende Hypothesen gewählt:

1. Die Initiatoreffektivität wird nur durch Käfigreaktionen herabgesenkt.

2. Radikale, die den Lösungsmittelkäfigen entkommen, starten ein Kettenwachstum.

3. Der Stoffmengenanteil der Käfigprodukte aus Initiator-Zerfallsexperimente in $n$-Heptan ist übertragbar auf den Stoffmengenanteil von Käfigprodukten bei der Ethen-Hochdruckpolymerisation.

Um die Käfigreaktionen modellieren zu können, muss vorweg ein kinetisches Modell aufgestellt und an experimentelle Daten angepasst werden.

Für mehrere tert-Butylperoxyester sind zum einen Initiatoreffektivitäten und zum anderen GC-Zerfallsproduktanalysen in $n$-Heptan vorhanden, so dass für diese Peroxyester ein kinetisches Modell aufgestellt und an vorhandene Daten angepasst wurde [1, 2, 3, 4, 5].

\subsection{Das kinetische Schema}

Die Käfigreaktionen (Kombination und Kreuz-Disproportionierung) sind bimolekulare Reaktionen. Sie sind somit abhängig von der makroskopischen Konzentration zweier Reaktanden. Da aber nur die Radikale im selben Lösungsmittelkäfig zu Käfigprodukten reagieren dürfen, wurden virtuelle Käfigspezies implementiert, die unimolekular zu den Produkten reagieren. Dies hat die Folge, dass eine unimolekulare Geschwindigkeitskonstante einer bimolekularen Reaktion zugeordnet wird. Bei einigen Käfigreaktionen müssen zusätzliche Reaktionsschritte eingeführt werden, die mit der hohen Geschwindigkeitskonstante von $k_{\text {fast }}=1 \cdot 10^{50} \mathrm{~s}^{-1}$ (der Wert wurde willkürlich gesetzt, musste aber bedeutend höher sein als die restlichen Geschwindigkeitskonstanten) erfolgen, da das Programm Predici 
bei einer unimolekularen Reaktion nur zwei Produktmoleküle entstehen lassen kann. Während der Käfigreaktionen können aber im Lösungsmittelkäfig mehr als zwei Moleküle gebildet werden, so dass eine schnelle Folgereaktion angenommen wird, in der eventuell weitere Produkte entstehen (siehe unten im kinetischen Schema).

Das in Predici implementierte kinetischer Schema lautet wie folgt:

\section{Zerfall und Rekombination}

Peroxyester $\stackrel{k_{\text {diss }}}{\longrightarrow}[\text { Alkoxyradikal }+ \text { Acyloxyradikal }]_{\text {cage } 1}$

$[\text { Alkoxyradikal }+ \text { Acyloxyradikal }]_{\text {cage } 1} \stackrel{k_{\text {rec }}}{\longrightarrow}$ Peroxyester

\section{Käfigreaktion}

$[\text { Alkoxyradikal }+ \text { Acyloxyradikal }]_{\text {cage } 1} \stackrel{k \mathrm{co} 2}{\longrightarrow}\left[\text { Alkoxyradikal }+ \text { Alkylradikal }+\mathrm{CO}_{2}\right]_{\text {cage2 }}$

$\left[\text { Alkoxyradikal }+ \text { Alkylradikal }+\mathrm{CO}_{2}\right]_{\text {cage2 }} \stackrel{k_{\mathrm{comb}}}{\longrightarrow}$ Ether $+\mathrm{CO}_{2}$

$\left[\text { Alkoxyradikal }+ \text { Alkylradikal }+\mathrm{CO}_{2}\right]_{\text {cage2 }} \stackrel{k_{\text {cross }}}{\longrightarrow}\left[\text { Alken }+\mathrm{CO}_{2}\right]_{\text {cage2,cross }}+$ Alkohol

$\left[\text { Alken }+\mathrm{CO}_{2}\right]_{\text {cage2,cross }} \stackrel{k_{\text {fast }}}{\longrightarrow} \mathrm{CO}_{2}+$ Alken

$\underline{\text { Diffusion }}$

$[\text { Alkoxyradikal }+ \text { Acyloxyradikal }]_{\text {cage } 1} \stackrel{k_{\text {diff }}}{\longrightarrow}$ Alkoxyradikal + Acyloxyradikal

$\left[\text { Alkoxyradikal }+ \text { Alkylradikal }+\mathrm{CO}_{2}\right]_{\text {cage2 }} \stackrel{k_{\text {diff }}}{\longrightarrow}$

$\left[\text { Alkylradikal }+\mathrm{CO}_{2}\right]_{\text {cage2,Rest }}+$ Alkoxyradikal

$\left[\text { Alkylradikal }+\mathrm{CO}_{2}\right]_{\text {cage2,Rest }} \stackrel{k \mathrm{fast}}{\longrightarrow} \mathrm{CO}_{2}+$ Alkylradikal

\section{$\underline{\text { Reaktionen außerhalb des Käfigs }}$}

Alkoxyradikal $\stackrel{k_{\beta}}{\longrightarrow}$ Aceton + Alkylradikal

Alkoxyradikal + Heptan $\stackrel{k_{o l}}{\longrightarrow}$ Alkohol + Heptylradikal

In Abbildung 5.1 ist das kinetische Schema veranschaulicht. Zunächst zerfällt der tert-Butylperoxyester in zwei sauerstoffzentrierte Radikale, die entweder zum Ausgangsmolekül rekombinieren oder weiterzerfallen können. Die $\beta$-scission des tert-Butoxyradikals erfolgt nur 
außerhalb des Lösungsmittelkäfigs (siehe Kapitel 4.11.2) in Konkurrenz zur H-Abstraktion vom Lösungsmittel. Die Decarboxylierung erfolgt anteilig im Käfig abhängig vom Rest $\mathrm{R}_{1}$. Die im Käfig gebildeten tert-Butoxy- und Alkylradikale können nun aus dem Lösungsmittelkäfig diffundieren, zum Ether kombinieren oder zum tert-Butanol und Alken kreuzdisproportionieren. Ether, Aceton und tert-Butanol wurden als Zersetzungsprodukte beim thermischen Zerfall der tert-Butylperoxyester gefunden [4,5]. Die Stoffmengenanteile der Zersetzungsprodukte dienen zur Anpassung der Geschwindigkeitskonstanten der Käfigreaktionen (Kombination und Kreuz-Disproportionierung).
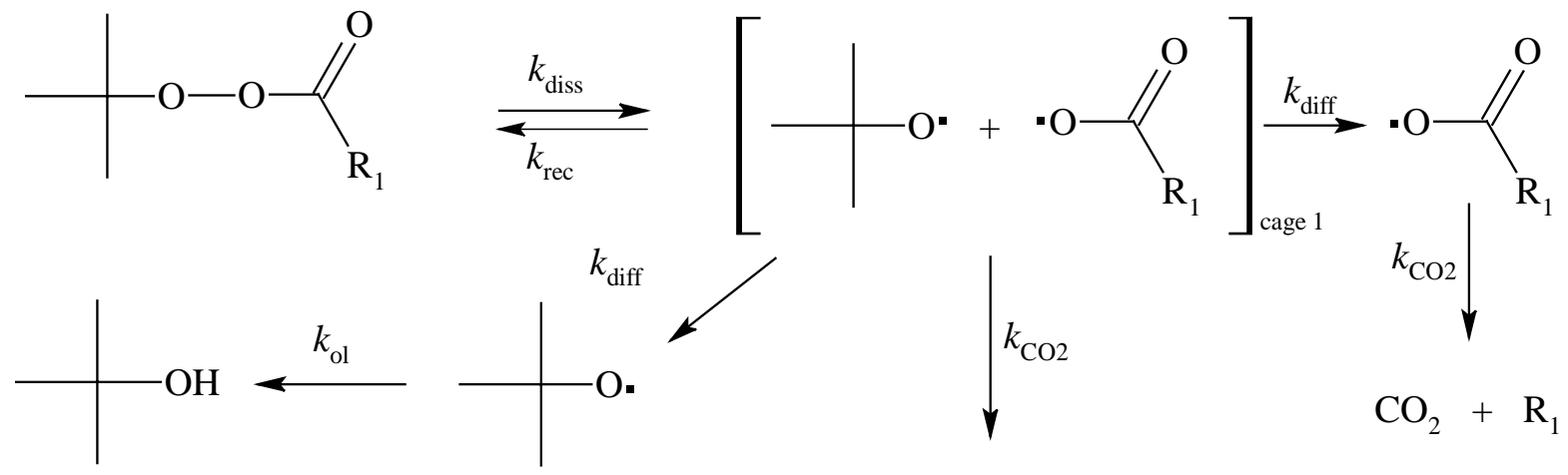

diff
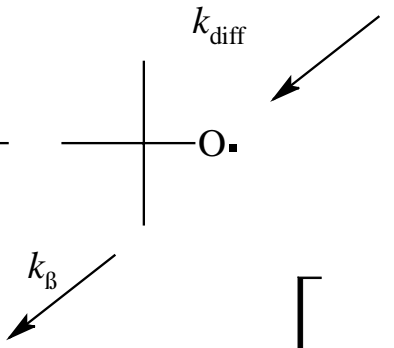<smiles>C[C+]C(C)=O</smiles>
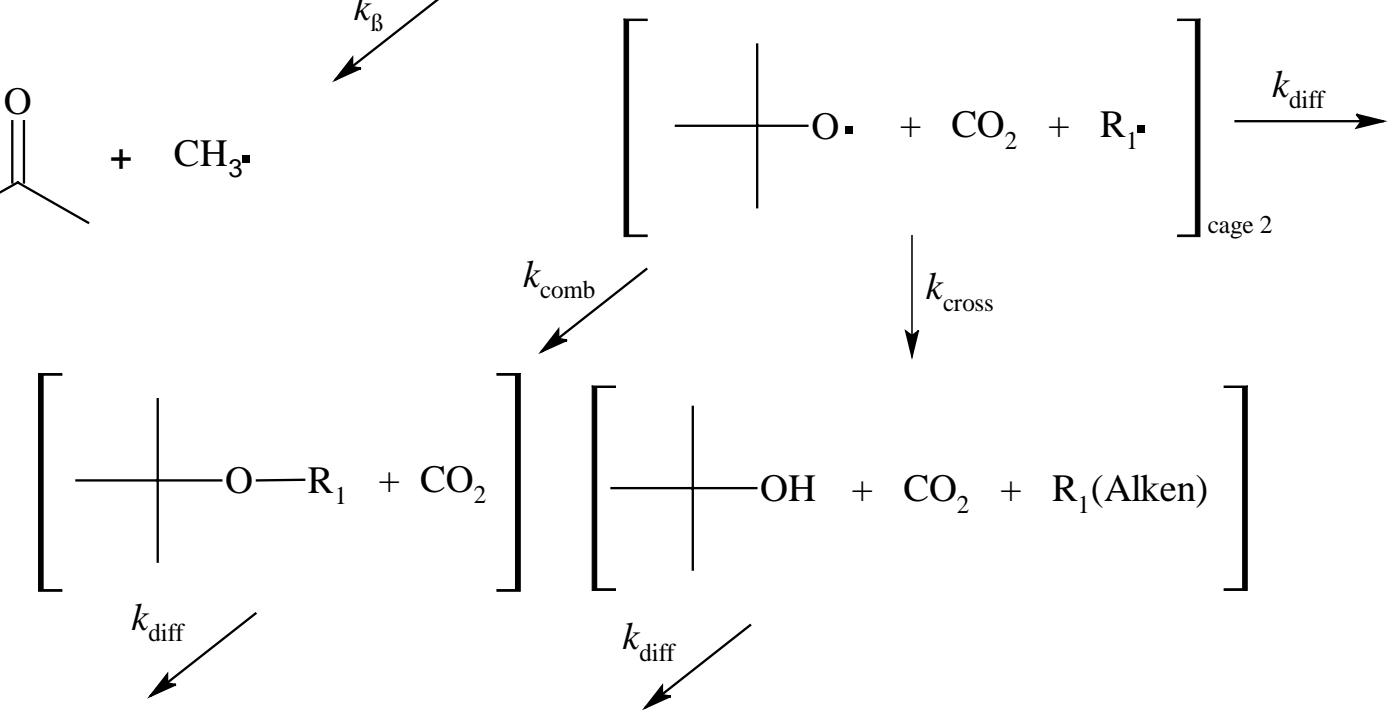

Abb. 5.1: Das kinetische Schema zur Modellierung mit Strukturformeln dargestellt. Die eckigen Klammern stellen den Lösungsmittelkäfig dar. 


\subsection{Die Geschwindigkeitskonstanten}

Für die Teilreaktionen im oben dargestellten Schema und Abbildung 5.1 benötigt man Geschwindigkeitskonstanten. Diese wurden, soweit vorhanden, aus der Literatur entnommen, berechnet oder in Predici angepasst.

\subsubsection{Die Diffusionsgeschwindigkeitskonstante $\boldsymbol{k}_{\text {diff }}$}

Es wurde für jeden Diffusionsschritt dieselbe Diffusionsgeschwindigkeitskonstante $k_{\text {diff }}$ angenommen. Diese wurde wie in der Arbeit von WITTKOWSKI aus $n$-Heptandichtedaten bestimmt [6]. Zunächst wurde aus den temperatur- und druckabhängigen Dichtedaten von DoolitTle [7] die Viskosität nach der Stiel-Thodos-Methode berechnet [8]. Die Formel lautet:

$$
\begin{array}{r}
{\left[\left(\eta-\eta_{0}\right) \xi+10^{-4}\right]^{0.25}=0.10230+0.023364 \rho_{r}+0.058533 \rho_{r}{ }^{2}} \\
-0.040758 \rho_{r}{ }^{3}+0.0093324 \rho_{r}{ }^{4}
\end{array}
$$

mit

$\eta \quad=$ Viskosität in $\mathrm{cP}$

$\eta_{0} \quad=$ Viskosität bei verschwindenden Druck bei der gleichen Temperatur in cP

$\eta_{0} \xi \quad=34.0 \cdot 10^{-5} T_{r}^{0.94}$ für $T_{r} \leq 1.5$

$\eta_{0} \xi=17.78 \cdot 10^{-5}\left(4.58 T_{r}-1.67\right)^{5 / 8}$ für $T_{r}>1.5$

$T_{r} \quad=$ reduzierte Temperatur, $T / T_{c}$

$\xi \quad=\left(\frac{T_{c}}{K}\right)^{1 / 6} /\left[\left(\frac{M}{\mathrm{~g} \mathrm{~mol}^{-1}}\right)^{1 / 2} \cdot\left(\frac{P_{c}}{\mathrm{~atm}}\right)^{2 / 3}\right]$

$\rho_{r} \quad=$ reduzierte Dichte, $\rho / \rho_{c}$

für $n$-Heptan ist:

$T_{c} \quad=540.3 \mathrm{~K}$

$P_{c} \quad=27.0 \mathrm{~atm}$

$M \quad=100.2 \mathrm{~g} \mathrm{~mol}^{-1}$ 
Die Diffusionskonstante wurden aus den Viskositätsdaten mit der Wilke-Chan-Gleichung [8] berechnet:

$$
D_{12}=7.4 \cdot 10^{-8}\left[\left(\phi M_{2}\right)^{1 / 2} T /\left(\eta_{2} V_{1}^{0.6}\right) \mid\right.
$$

mit

$D_{12}=$ Diffusionskoeffizient des Gelösten 1 im Lösungsmittel 2 in $\mathrm{cm}^{2} \mathrm{~s}^{-1}$

$\phi \quad=$ Assoziationsparameter des Lösungsmittels, für $n$-Heptan 1.0

$\eta_{2} \quad=$ Viskosität des Lösungsmittels in $\mathrm{cP}$

$V_{1}=$ Molares Volumen des Gelösten bei dessen Siedepunkt in $\mathrm{cm}^{3} \mathrm{~mol}^{-1}$ abgeschätzt durch die Inkrementmethode von LeBas [8]

$\mathrm{H}=3.7 \mathrm{~cm}^{3} \mathrm{~mol}^{-1}, \mathrm{C}=14.8 \mathrm{~cm}^{3} \mathrm{~mol}^{-1}, \mathrm{O}=7.4 \mathrm{~cm}^{3} \mathrm{~mol}^{-1}$ als Modellsubstanz wurde das tert-Butoxyradikal verwendet

Mit dem Diffusionsmodell von Einstein-Smoluchowski wurde $k_{\text {diff }}$ berechnet.

$$
k_{\text {diff }}=\tau^{-1}=2 D_{12} \lambda^{-2}
$$

mit

$\tau \quad=$ benötigte Zeit um die Diffusionsstrecke $\lambda$ zu überwinden

$\lambda=$ Diffusionsstrecke

Im Modell wird angenommen, dass im zeitlichen Mittel ein Lösungsmittelmolekül - in diesem Fall ein $n$-Heptanmolekül - kugelförmig ist. Weiter wird angenommen, dass, wenn sich zwischen zwei Moleküle ein kugelförmiges $n$-Heptanmolekül befindet, diese Moleküle diffusiv getrennt sind. Dies bedeutet, dass die Diffusionsstrecke $\lambda$, die ein Molekül zurücklegen muss, damit der Käfig zerfallen ist, dem Durchmesser des kugelförmigen n-Heptanmoleküls entspricht. Aus der Dichte, der Molmasse und dem Kugelvolumen lässt sich die Diffusionsstrecke berechnen.

\subsubsection{Die Rekombinationsgeschwindigkeitskonstante $\boldsymbol{k}_{\text {rec }}$}

NEUMAN und BusSEY haben für tert-Butoxyradikale das Verhältnis von $k_{\text {diff }}$ und $k_{\text {rec }}$ in Abhängigkeit vom Druck bei $45^{\circ} \mathrm{C}$ in $n$-Octan bestimmt [9]. Für $k_{\text {rec }}$ wird in der Literatur 
keine Temperaturabhängigkeit angenommen [10]. Da $k_{\text {diff }}$ über die oben beschriebene Methode berechnet werden kann, ist die Bestimmung von $k_{\text {rec }}$ über das Verhältnis möglich. Aus der Druckabhängigkeit des Verhältnisses $k_{\text {diff }}$ und $k_{\text {rec }}$ wurde eine druck- und temperaturunabhängige Rekombinationsgeschwindigkeitskonstante $k_{\text {rec }}=2.6 \cdot 10^{9} \mathrm{~s}^{-1}$ erhalten .

\subsubsection{Die Dissoziationsgeschwindigkeitskonstante $\boldsymbol{k}_{\text {diss }}$}

Für den thermischen Bindungsbruch der Peroxidbindung ist in der Literatur für die untersuchten Peroxyester die beobachteten Geschwindigkeitskonstanten $k_{\text {obs }}$ verfügbar $[11,12]$. PRYOR hat gezeigt, dass diese beobachtbare Geschwindigkeitskonstante von dem primären Bindungsbruch, der Diffusion, der Decarboxylierung, der $\beta$-scission und der Rekombination abhängt (siehe Gleichung 5.3) [13].

$$
k_{\mathrm{obs}}=k_{\mathrm{diss}}\left(\frac{k_{\mathrm{diff}}+k_{\mathrm{CO} 2}+k_{\beta}}{k_{\mathrm{diff}}+k_{\mathrm{CO} 2}+k_{\beta}+k_{\mathrm{rec}}}\right)
$$

Sind die Geschwindigkeitskonstanten für diese Reaktionen bekannt, kann $k_{\text {diss }}$ mit folgender Gleichung berechnet werden:

$$
k_{\mathrm{diss}}=k_{\mathrm{obs}}\left(\frac{k_{\mathrm{diff}}+k_{\mathrm{CO} 2}+k_{\beta}+k_{\mathrm{rec}}}{k_{\mathrm{diff}}+k_{\mathrm{CO} 2}+k_{\beta}}\right)
$$

\subsubsection{Die $\beta$-scission-Geschwindigkeitskonstante $k_{\beta}$}

Für die $\beta$-scission des tert-Butoxy-Radikals wurden auf Geschwindigkeitskonstanten zurückgegriffen, die mit Hilfe von DFT-Berechnungen bestimmt wurden [14] (siehe Kapitel 4.10.2). Mit den optimierten Geometrien des Grundzustandes und des Übergangzustandes aus den oben genannten DFT-Berechnungen wurde das Aktivierungsvolumen abgeschätzt. Aus den Unterschieden der einzelnen Bindungslängen wurde die Volumenänderung zwischen Übergangszustand und Grundzustand mit Gleichung 5.5 berechnet [15]. 
$\Delta V^{\ddagger}=\frac{\pi\left(r_{\mathrm{A}}^{2}+r_{\mathrm{B}}^{2}\right)}{2} \cdot \Delta l$

mit

$r$ = van-der-Waals-Radius des Atoms in der jeweiligen Bindung [16]

$\Delta l=$ Änderung der Bindungslänge beim Übergang vom Edukt zum Übergangszustand

Die Summe dieser Volumenänderungen wurde dem Aktivierungsvolumen gleich gesetzt. Für das Aktivierungsvolumen der $\beta$-scission wurde ein Wert von $2.2 \mathrm{~cm}^{3} \mathrm{~mol}^{-1}$ erhalten.

\subsubsection{Die Decarboxylierungsgeschwindigkeitskonstante $\boldsymbol{k}_{\mathrm{CO} 2}$}

In der Literatur ist für das Acetyloxy- und Propionyloxyradikal eine Temperaturabhängigkeit von $k_{\mathrm{CO} 2}$ erhältlich [17]. BRAUN, RAJBENBACH und EIRICH haben für das Acetyloxyradikal eine Geschwindigkeitskonstante von $1.6 \cdot 10^{9} \mathrm{~s}^{-1}$ bei $60{ }^{\circ} \mathrm{C}$ abgeschätzt [18]. Für das Propionyloxy-, iso-Butyryloxy- und Pivalyloxyradikal wird eine Geschwindigkeitskonstante bei $20^{\circ} \mathrm{C}$ angegeben [19], die durch Photolyse der Naphtyloxyderivate erhalten wurde. In Abbildung 5.2 sind alle Literaturwerte in einer Arrhenius-Auftragung dargestellt. Die Abbildung 5.2 zeigt die Streuung der Daten. Es ist nicht klar, weshalb $k_{\mathrm{CO} 2}$ des Acetyloxyradikals sehr viel kleiner ist als $k_{\mathrm{CO} 2}$ des Propionyloxyradikal. Auch ist nicht nachvollziehbar, dass die Decarboxylierung des iso-Butyryloxy- und Pivaloxyradikals, die zu dem sekundären und tertiären kohlenstoffzentrierten Radikalen führen, nicht wesentlich schneller sind.

Deshalb wurden für das Aetyloxy-, iso-Butyryloxy- und Pivalyloxyradikal DFT-Rechnung von Schmatz und KLING durchgeführt [20]. Für das Acetyloxyradikal waren die Berechnungen erfolgreich. Die Temperaturabhängigkeit von $k_{\mathrm{CO} 2}$ wird durch folgende Gleichung beschrieben:

$$
k_{\mathrm{CO} 2}=1.5 \cdot 10^{13} \exp \left(-\frac{20.92 \mathrm{~kJ} \mathrm{~mol}^{-1}}{R T}\right)
$$

Hingegen waren die Berechnungen für das iso-Butyryloxy- und Pivalyloxyradikal schwierig, weil die Decarboxylierung dieser Radikale über eine verschwindend kleine Barriere verläuft. 
Deshalb wurde die Decarboxylierungskinetik für das iso-Butyryloxy- und das Pivalyloxyradikal aus der Kinetik des Acetyloxyradikal abgeschätzt. Die Decarboxylierung zu einem tertiären Radikal sollte schneller erfolgen als die zu einem sekundären Radikal, so dass der präexponentielle Faktor beibehalten und die Aktivierungsenergie für das iso-Butyryloxyradikal zu $5 \mathrm{~kJ} \mathrm{~mol}^{-1}$ und für das Pivalyloxyradikal zu $1 \mathrm{~kJ} \cdot \mathrm{mol}^{-1}$ gesetzt wurde. Für die Decarboxylierung des n-Butyryloxyradikals wurde die Kinetik des Acetyloxyradikals angenommen.

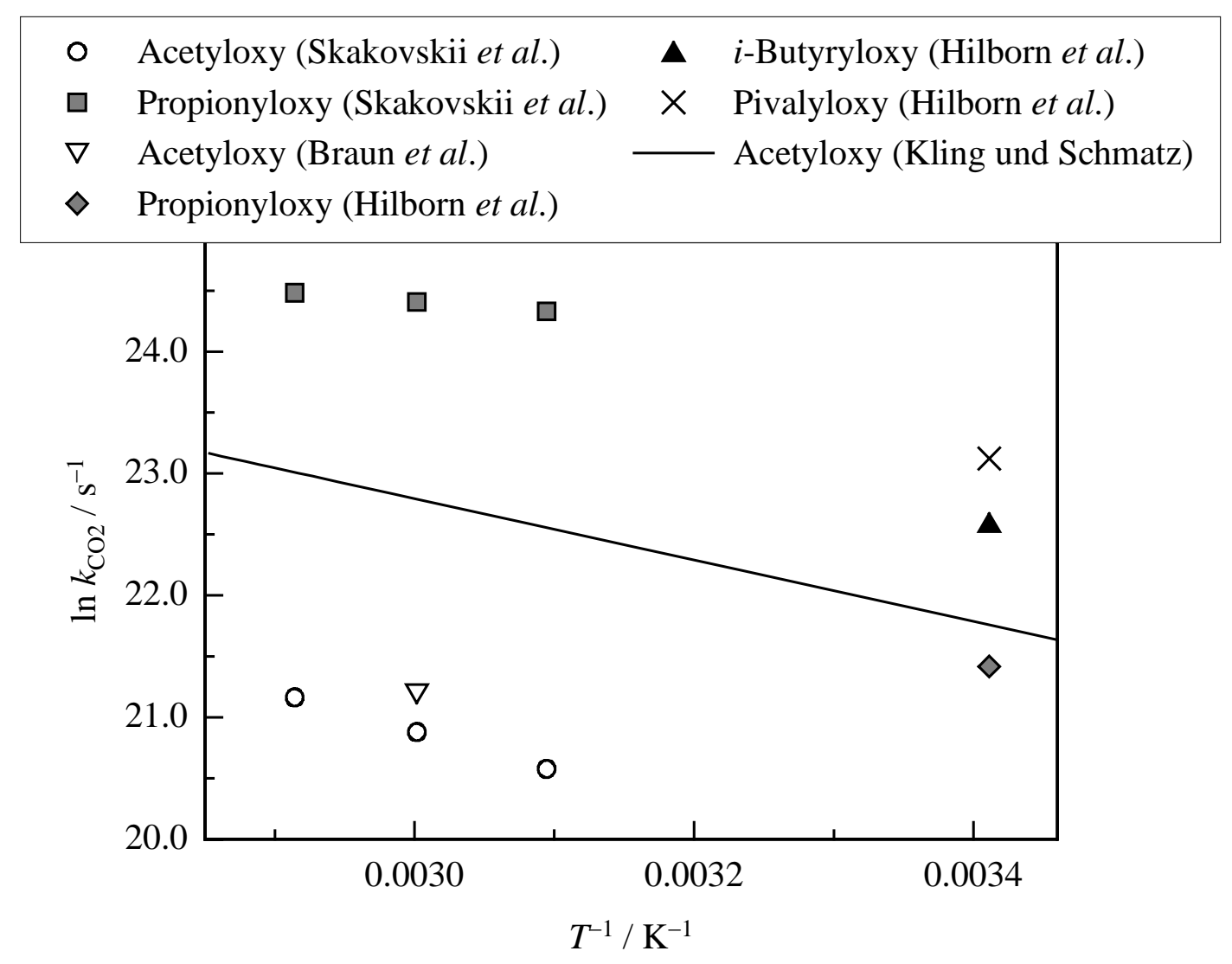

Abb.5.2: Temperaturabhängigkeit der Geschwindigkeitskonstante für die Decarboxylierung der Acyloxyradikale aus der Literatur [17, 18, 19 20].

Das Aktivierungsvolumen für die Decarboxylierung des Acetyloxyradikals wurde mit der gleichen Methode wie bei der $\beta$-scission abgeschätzt (siehe Kapitel 5.3.4). Es wurde ein Wert von $0.77 \mathrm{~cm}^{3} \cdot \mathrm{mol}^{-1}$ erhalten. Dieser Wert ist für eine unimolekulare Reaktion relativ klein, da die Bindungsdehnung der C-C-Bindung nahezu vollständig durch die Kontraktion der beiden C-O-Bindungen kompensiert wird. Für die anderen im Rahmen dieser Arbeit untersuchten Acyloxyradikale wurde derselbe Wert benutzt. 


\subsubsection{Die H-Abstraktionsgeschwindigkeitskonstante $\boldsymbol{k}_{\mathrm{ol}}$}

Die bimolekulare Geschwindigkeitskonstante $k_{\mathrm{ol}}$ wurde an die Alkoholkonzentrationen, die in den Zerfallsexperimenten des tert-Butylperoxyacetat in $n$-Heptan erhalten wurden, angepasst. Der Alkohol kann nur außerhalb des Käfigs gebildet werden, weil von dem Methylradikal, das nach der Decarboxylierung gebildet wird, kein H-Atom abstrahiert werden kann. Diese Geschwindigkeitskonstanten wurden für die restlichen tert-Butylperoxyester verwendet.

\subsubsection{Die Kombinationsgeschwindigkeitskonstante $\boldsymbol{k}_{\text {comb }}$ und die Kreuzdisproportionierungsgeschwindigkeitskonstante $\boldsymbol{k}_{\text {cross }}$}

Die Käfigreaktionsgeschwindigkeitskonstanten der Kombination $k_{\text {comb }}$ und der Kreuz-Disproportionierung $k_{\text {cross }}$ wurden wie $k_{\mathrm{ol}}$ mit Predici an die Käfigprodukte (Ether bzw. zusätzlicher Alkohol) aus den Zerfallsexperimenten angepasst.

\subsection{Ergebnisse der Modellierung der Käfigreaktionen von tert-Butylperoxyestern}<smiles>CC(=O)OOC(C)(C)C</smiles>
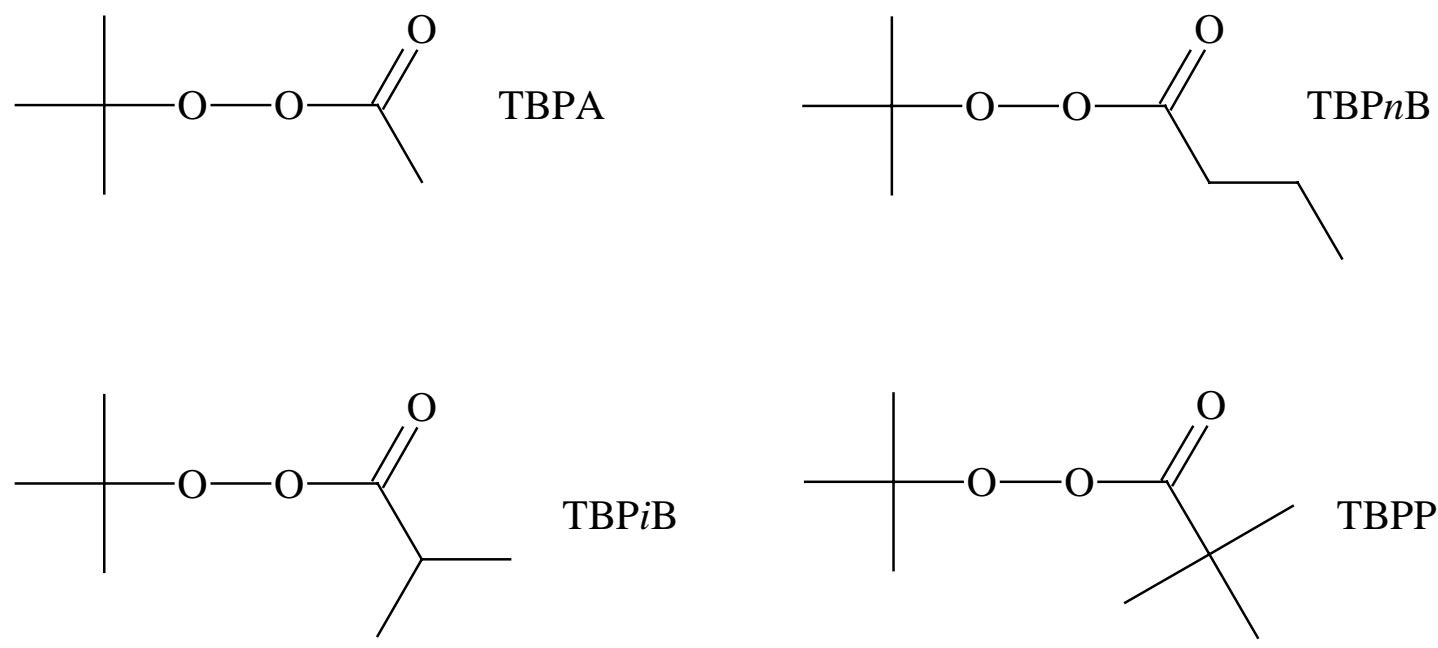

TBPiB

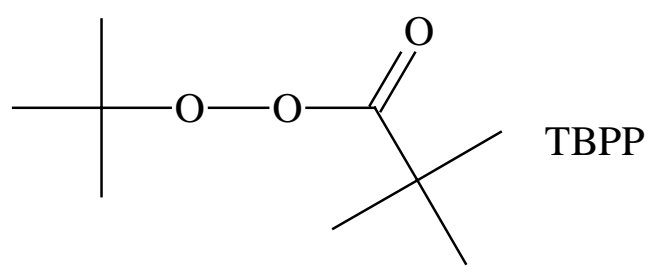

Abb. 5.3 Strukturformel der untersuchten tert-Butylperoxyester.

In diesem Kapitel werden die Temperatur- und Druckabhängigkeiten der modellierten Geschwindigkeitskonstanten für die Käfigreaktionen und die H-Abstraktion vom Lösungsmittel vorgestellt. Es werden für tert-Butylperoxyacetat (TBPA), tert-Butylperoxy-nbutyrat (TBPnB), tert-Butylperoxy-iso-butyrat (TBPiB) und tert-Butylperoxypivalat (TBPP) 
Geschwindigkeitskonstanten angegeben. Die Strukturformeln der tert-Butylperoxyester sind in Abbildung 5.3 gezeigt.

\subsubsection{Modellierung der Temperatur- und Druckabhängigkeit der Geschwindigkeitskonstanten für die H-Abstraktion von $n$-Heptan und die Kombination des tert-Butoxy- und Methylradikals}

Wie in Kapitel 5.3.6 erwähnt wurde, wurde zunächst $k_{\mathrm{ol}}$ und $k_{\mathrm{comb}}$ an Stoffmengenanteile der Zerfallsprodukte tert-Butanol und tert-Butylmethylether des TBPA angepasst [4]. Da keine Kreuzdisproportionierung auftreten kann, muss der Alkohol, der bei den Zerfallsexperimenten entstanden ist, komplett außerhalb des Käfigs gebildet worden sein, indem die tert-Butoxyradikale aus dem Käfig diffundiert sind und vom Lösungsmittel $n$-Heptan ein H-Atom abstrahiert haben. $k_{\mathrm{ol}}$ wurde an die entstandene Menge an tert-Butanol mit Predici angepasst. In Abbildung 5.4 ist die Temperaturabhängigkeit von $k_{\mathrm{ol}}$ bei 500 bar zu sehen. Die Aktivierungsenergie von $k_{\mathrm{ol}}$ ist $27.9 \mathrm{~kJ} \cdot \mathrm{mol}^{-1}$. Für die weiteren Peroxyester wurde dieses $k_{\mathrm{ol}}$ verwendet.

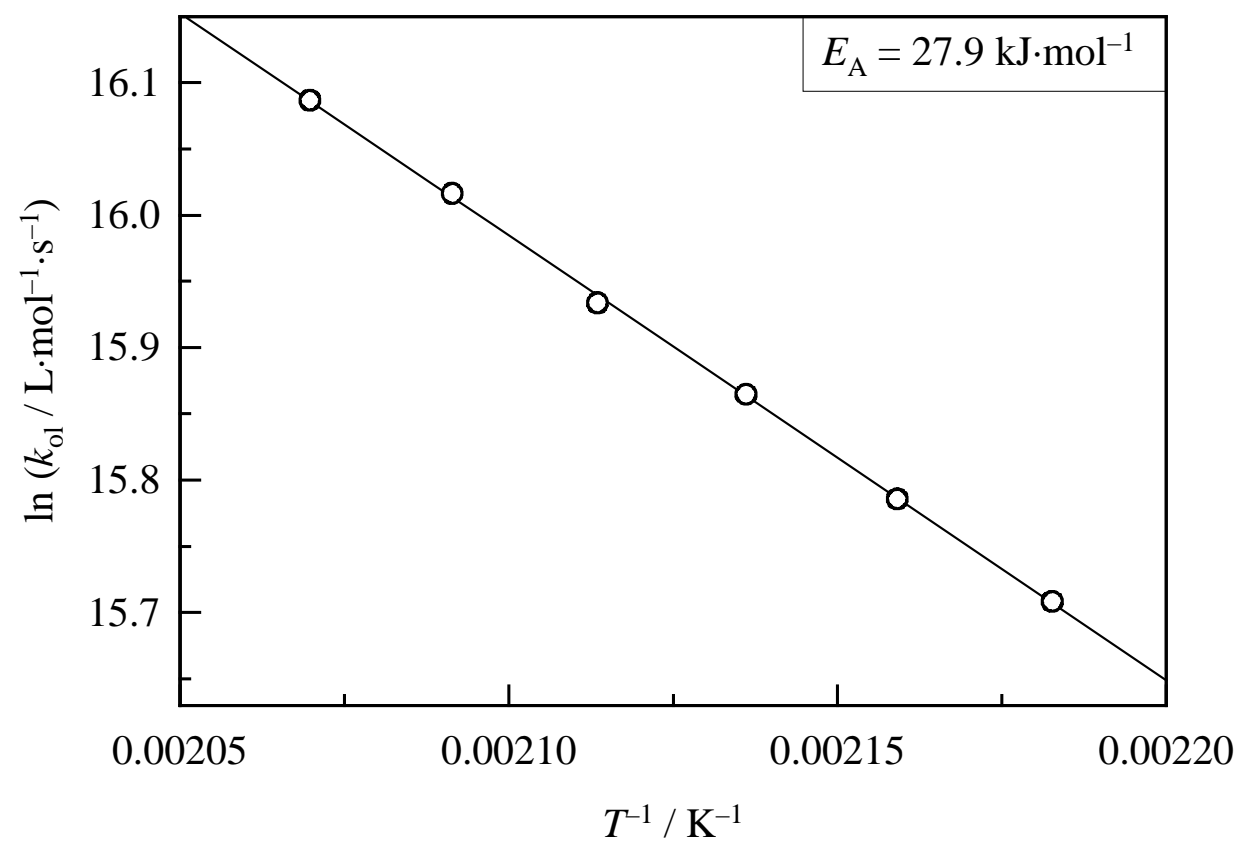

Abb.5.4: Erhaltende Temperaturabhängigkeit der Geschwindigkeitskonstante für die $H$-Abstraktion des tert-Butoxyradikals von n-Heptan bei 500 bar aus Anpassung an den Stoffmengenanteil von tert-Butanol.

An den gefundenen Ether wurde die Geschwindigkeitskonstante für die Kombination $k_{\text {comb }}$ mit Predici angepasst. Die Temperaturabhängigkeit ist in Abbildung 5.5 dargestellt. 


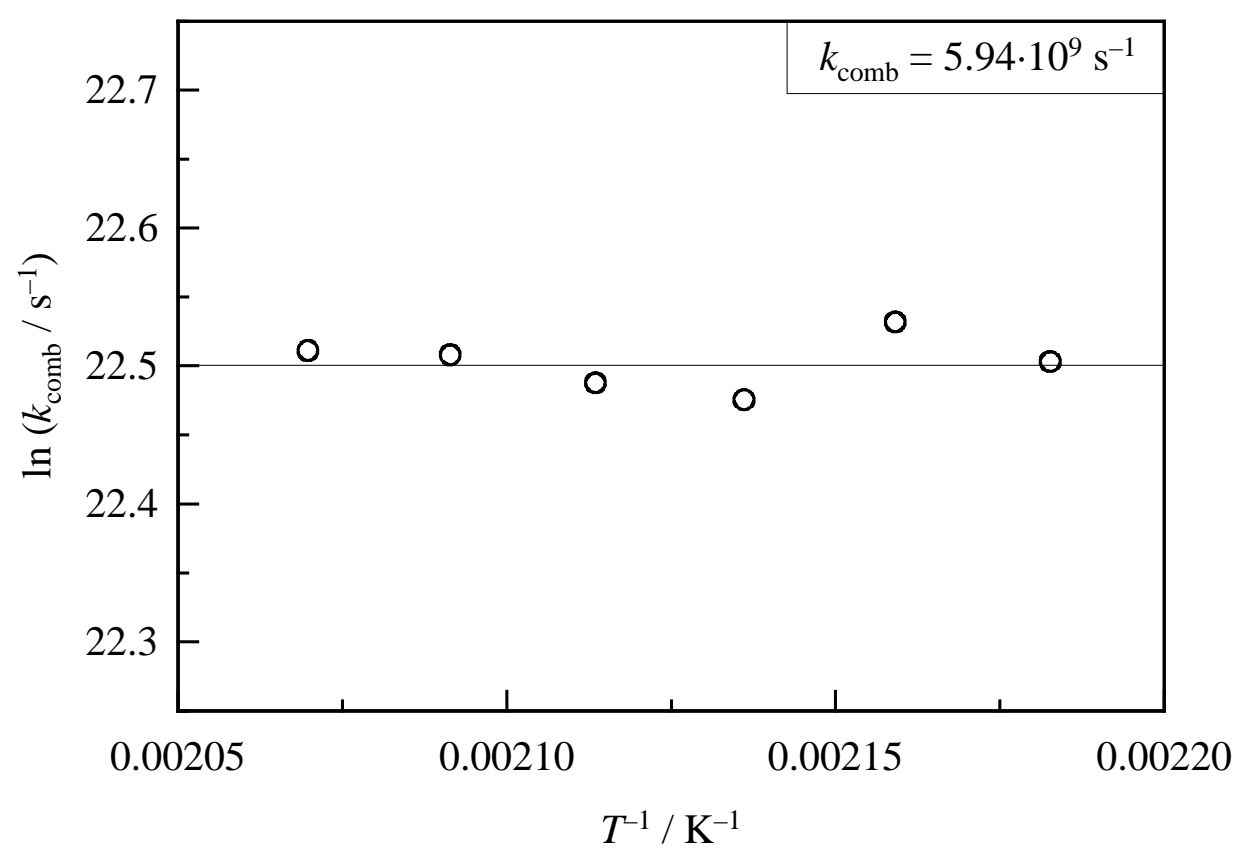

Abb. 5.5: Erhaltende Temperaturabhängigkeit der Geschwindigkeitskonstante für die Kombination des tert-Butoxyradikals und des Methylradikals bei 500 bar aus Anpassung an den Stoffmengenanteil von tert-Butylmethylether.

Es wird mit $k_{\text {comb }}$ von $5.94 \cdot 10^{9} \mathrm{~s}^{-1}$ eine temperaturunabhängige Geschwindigkeitskonstante $k_{\text {comb }}$ bestimmt. Für die weiteren tert-Butylperoxyester wurde kein entsprechender Ether gefunden. Es ist unwahrscheinlich, dass beim thermischen Zerfall kein Ether gebildet wird. Vielmehr ist wahrscheinlich, dass der Etherpeak im Gaschromatogramm unter dem Lösungmittelpeak verborgen war. Deshalb wurde mit diesem $k_{\text {comb }}$ für die weiteren tertButylperoxyester modelliert.

In Abbildung 5.6 ist die Druckabhängigkeit der Geschwindigkeitskonstante für die H-Abstraktion des tert-Butoxyradikals von $n$-Heptan gezeigt. Das erhaltende Aktivierungsvolumen $\Delta V^{\neq}$ist $-9.4 \mathrm{~cm}^{3} \cdot \mathrm{mol}^{-1}$. 


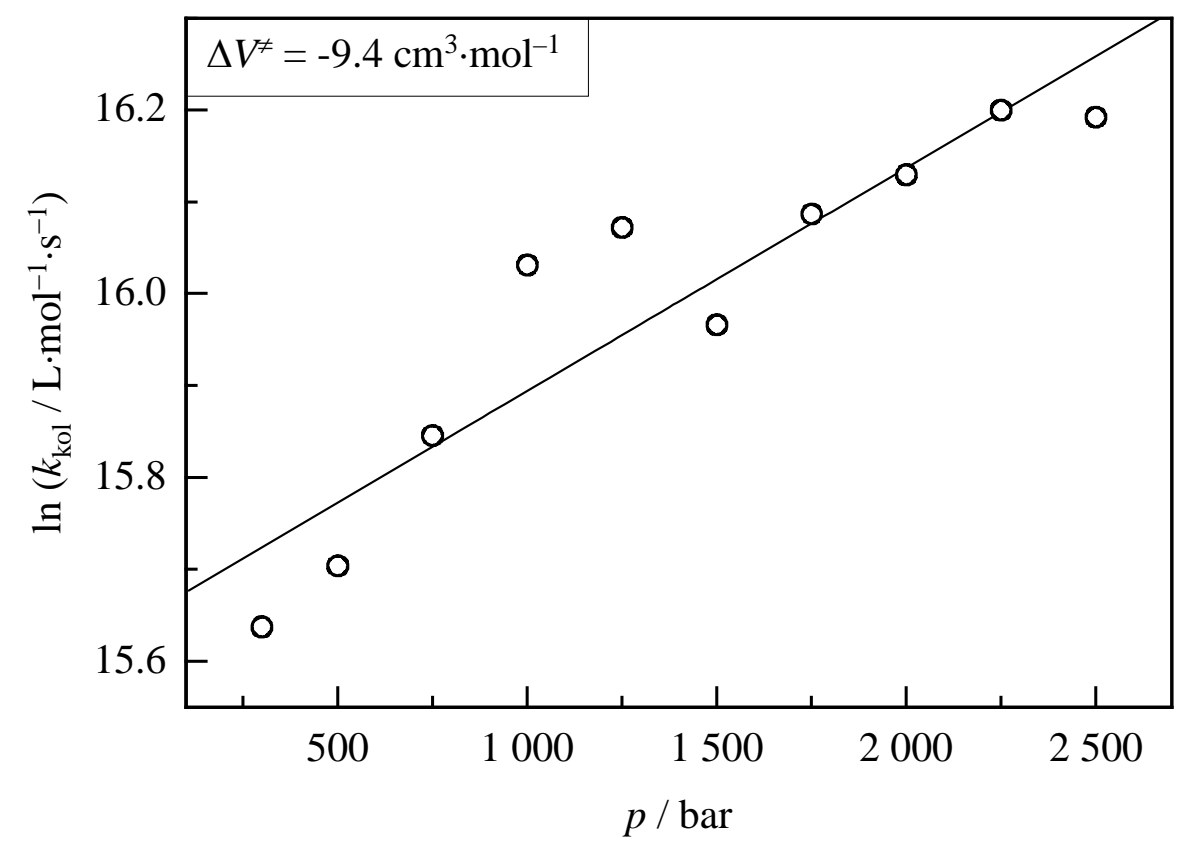

Abb. 5.6: Erhaltende Druckabhängigkeit der Geschwindigkeitskonstante für die $\mathrm{H}$ Abstraktion des tert-Butoxyradikals von n-Heptan bei $190{ }^{\circ} \mathrm{C}$ bar aus Anpassung an den Stoffmengenanteil von tert-Butanol.

Die Druckabhängigkeit der Kombinationsgeschwindigkeitskonstante $k_{\text {comb }}$ ist in Abbildung $5.7 \mathrm{zu}$ sehen.

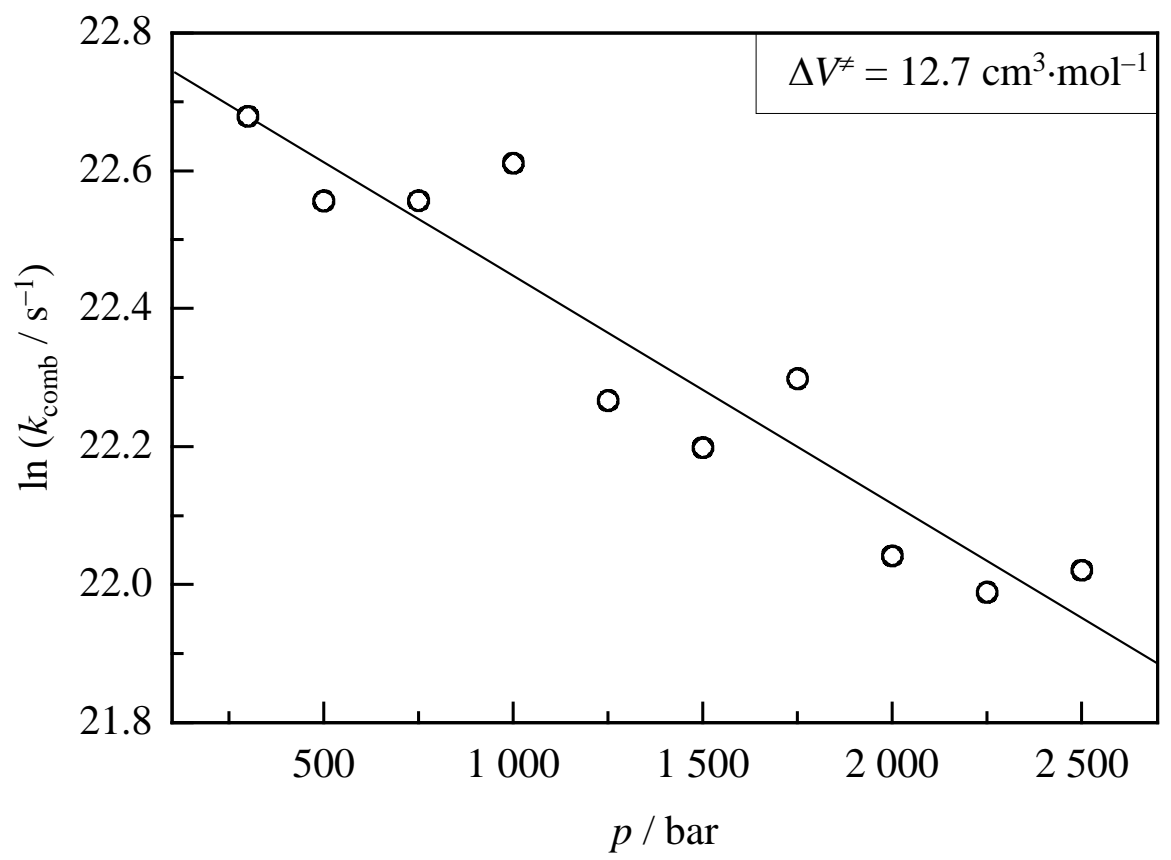

Abb.5.7: Erhaltende Druckabhängigkeit der Geschwindigkeitskonstante für die Kombination des tert-Butoxyradikals und des Methylradikals bei 190 bar aus Anpassung an den Stoffmengenanteil von tert-Butylmethylether. 


\subsubsection{Temperatur- und Druckabhängigkeit der Initiatoreffektivität abgeschätzt aus dem Stoffmengenanteil der Käfigprodukte für tert-Butylperoxyacetat}

Mit dem Modell werden neben den Geschwindigkeitskonstanten auch die Mengen an Käfigprodukten erhalten. Mit der Annahme, dass alle Käfigprodukte die Initiatoreffektivität absenken, lässt sich mit folgender Gleichung die Initiatoreffektivität $f$ berechnen.

$$
f=1-\Sigma x_{\text {Käfigprodukt }}
$$

$x_{\text {Käfigprodukte }}$ ist der Stoffmengenanteil der gefundenen Käfigprodukte bezogen auf die zersetzte Menge an Peroxid. Entstehen zwei Käfigprodukte bei einer Käfigreaktion (wie bei der Kreuzdisproportionierung), wird nur ein Käfigprodukt gezählt. Die Bedingungen der Zersetzungsexperimente wurden so gewählt, dass die tert-Butylperoxyester vollständig zersetzt waren.

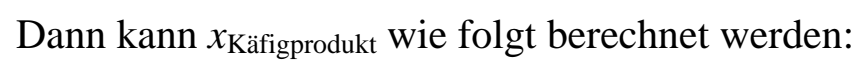

$$
X_{\text {Käfigprodukt }}=\frac{C_{\text {Käfigprodukt }}}{C_{\mathrm{INI}, 0}}
$$

In Abbildung 5.8 ist die Temperaturabhängigkeit der aus Käfigprodukten berechneten Initiatoreffektivität von TBPA bei 500 bar gezeigt. Die Effektivität ist temperaturunabhängig.

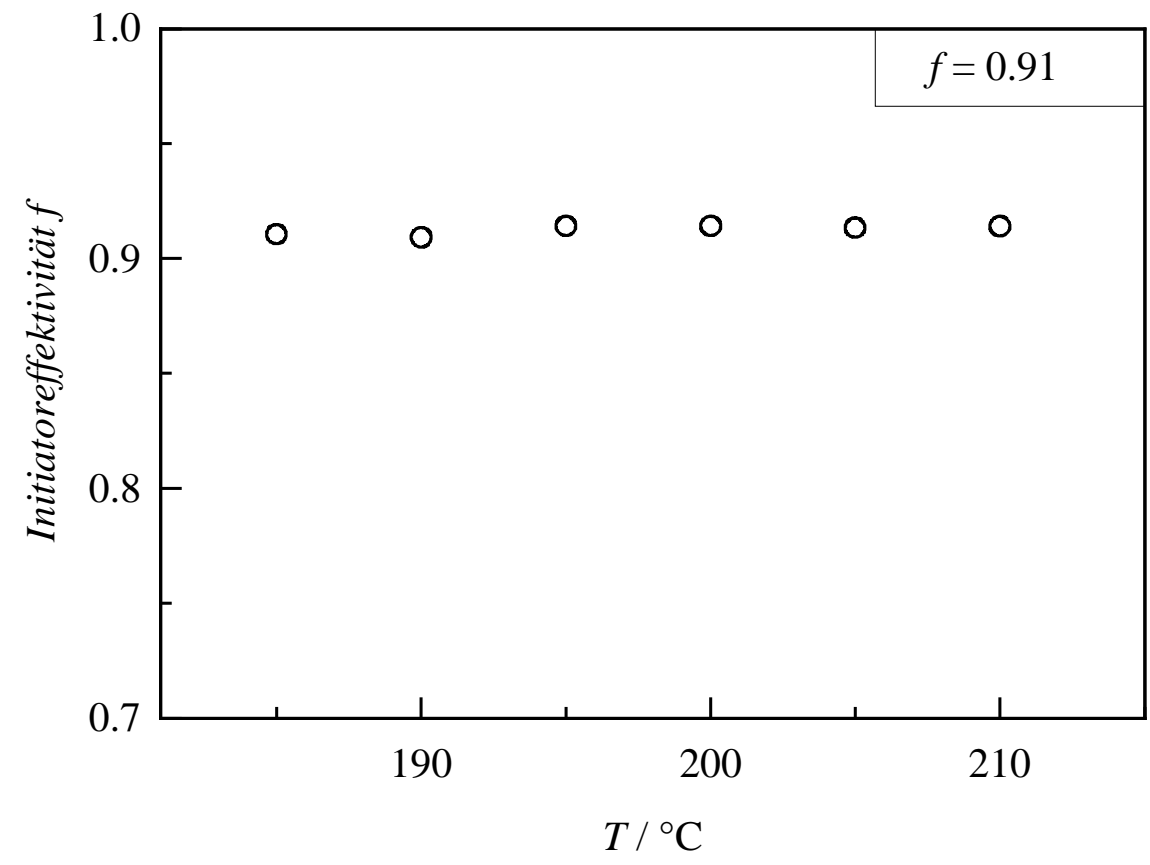

Abb. 5.8: Temperaturabhängigkeit der Initiatoreffektivität von TBPA berechnet aus $x_{E t h e r}$ bei 500 bar. 
Die berechnete Initiatoreffektivität weist eine Druckabhängigkeit auf, wie in Abbildung $5.9 \mathrm{zu}$ sehen ist. $f$ fällt von 0.92 bei 300 bar auf 0.76 bei 2500 bar. Der Wert für 2000 bar ( $f_{\text {Käfigprodukte }}$ $=0.81$ ) stimmt sehr gut mit dem aus der Ethen-Hochdruckpolymerisation erhaltenden Wert überein $\left(f_{\text {Ethen }}=0.79\right)$.

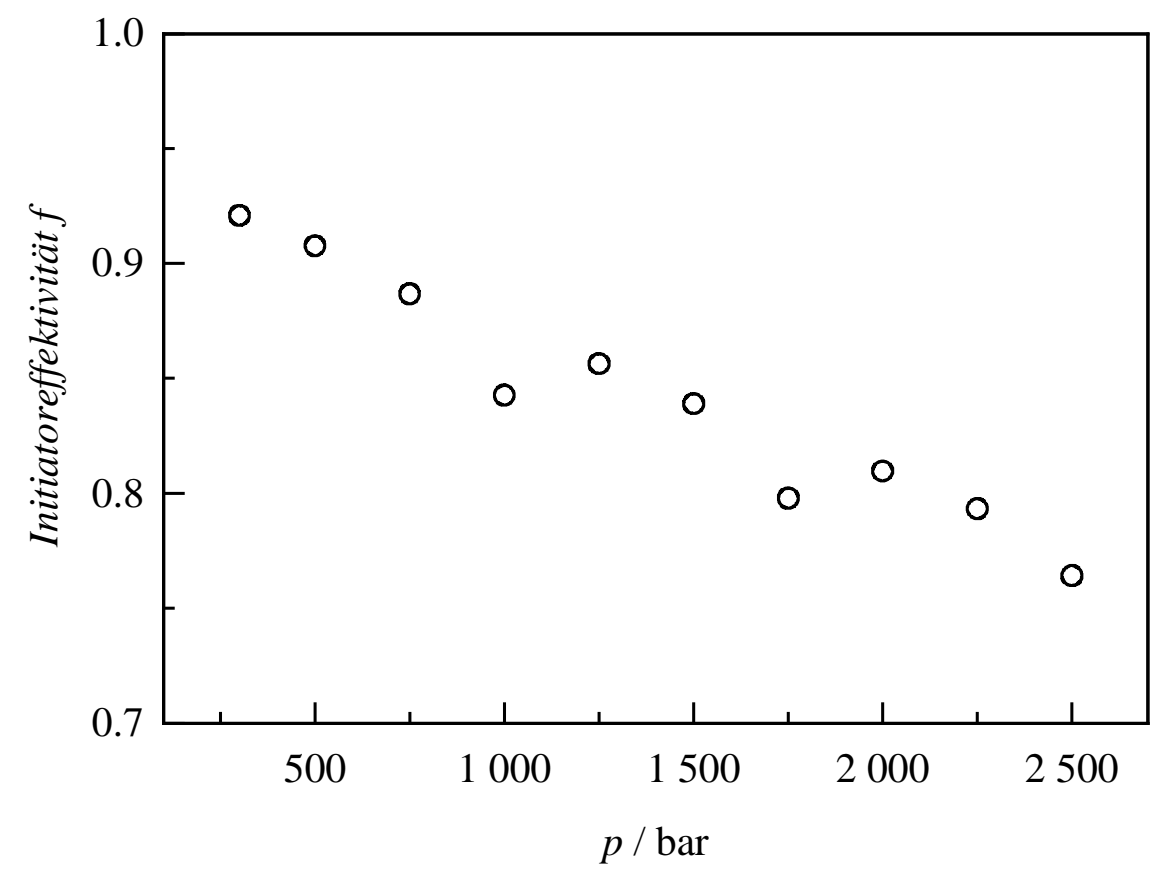

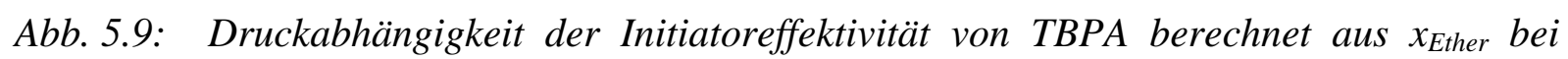
$190{ }^{\circ} \mathrm{C}$

\subsubsection{Modellierung der Temperatur- und Druckabhängigkeit der Geschwindigkeitskonstanten für die Kreuzdisproportionierung des tert-Butoxy- und n-Propylradikals}

Bis auf TBPA können die tert-Butylperoxyester im Käfig tert-Butanol bilden, das durch die Kreuzdisproportionierung des tert-Butoxyradikals mit dem Alkylradikal entsteht. Das Alkylradikal bildet sich bei der Decarboxylierung des Acyloxyradikals. Im Falle des TBPnB handelt es sich beim Alkylradikal um das n-Propylradikal. Die Temperatur- und Druckabhängigkeit der Geschwindigkeitskonstante für die Kreuzdisproportionierung ist in Abbildung 5.10 und 5.11 zu sehen. Die Geschwindigkeitskonstante $k_{\text {cross }}$ wurde durch Anpassung an den Stoffmengenanteil des überschüssigen tert-Butanols erhalten. Wie die Kombinationsgeschwindigkeitskonstante des tert-Butoxyradikals mit dem Methylradikal ist die Geschwindigkeitskonstante der Kreuzdisproportionierung ebenfalls temperaturunabhängig, aber sehr viel höher als $k_{\text {comb. }}$ Das Aktivierungsvolumen beträgt $24.6 \mathrm{~cm}^{3} \cdot \mathrm{mol}^{-1}$. Dies ist ein unerwartet hoher Wert. $k_{\mathrm{ol}}$ und $k_{\text {comb }}$ wurden von TBPA übernommen. 


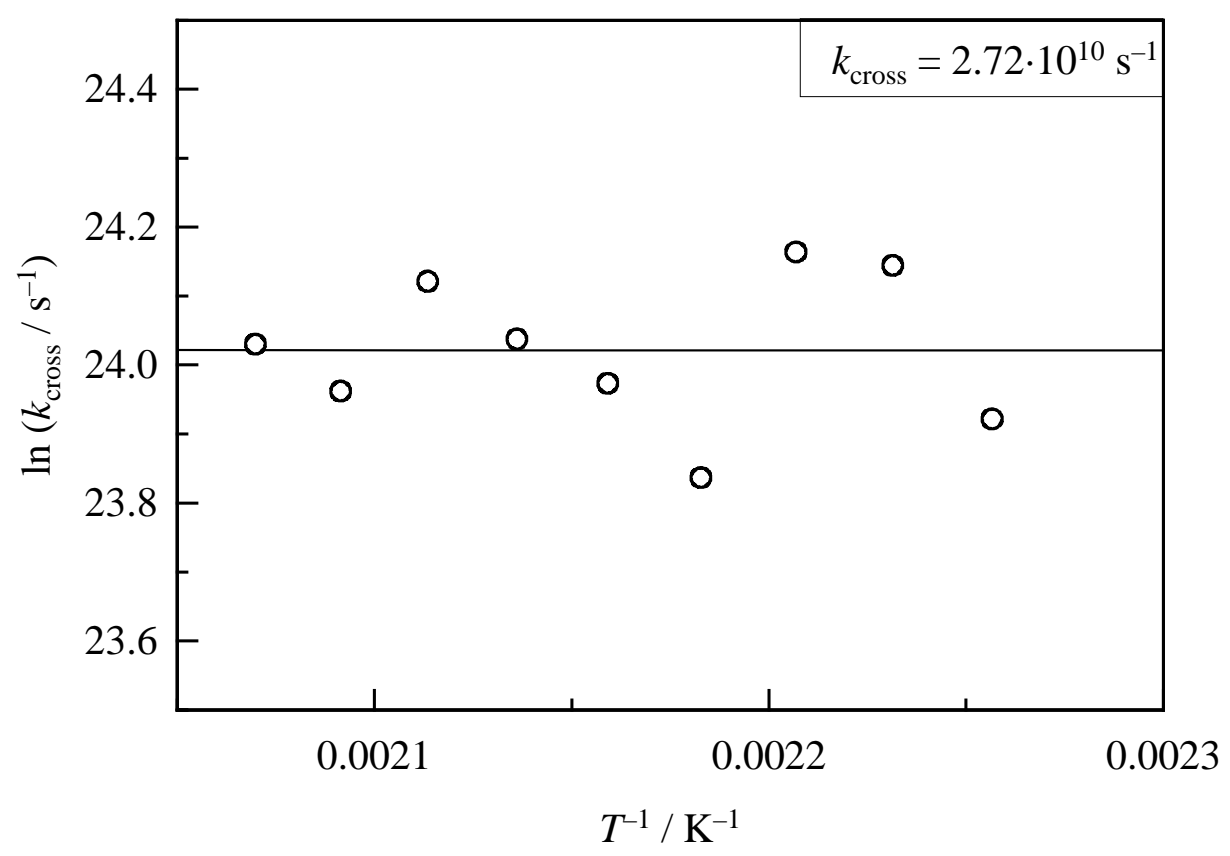

Abb. 5.10: Erhaltende Temperaturabhängigkeit der Geschwindigkeitskonstante für die Kreuzdisproportionierung des tert-Butoxyradikals und n-Propylradikals aus Anpassung an den Stoffmengenanteil von tert-Butanol bei 500 bar. $k_{\text {comb }}$ und $k_{o l}$ von TBPA übernommen.

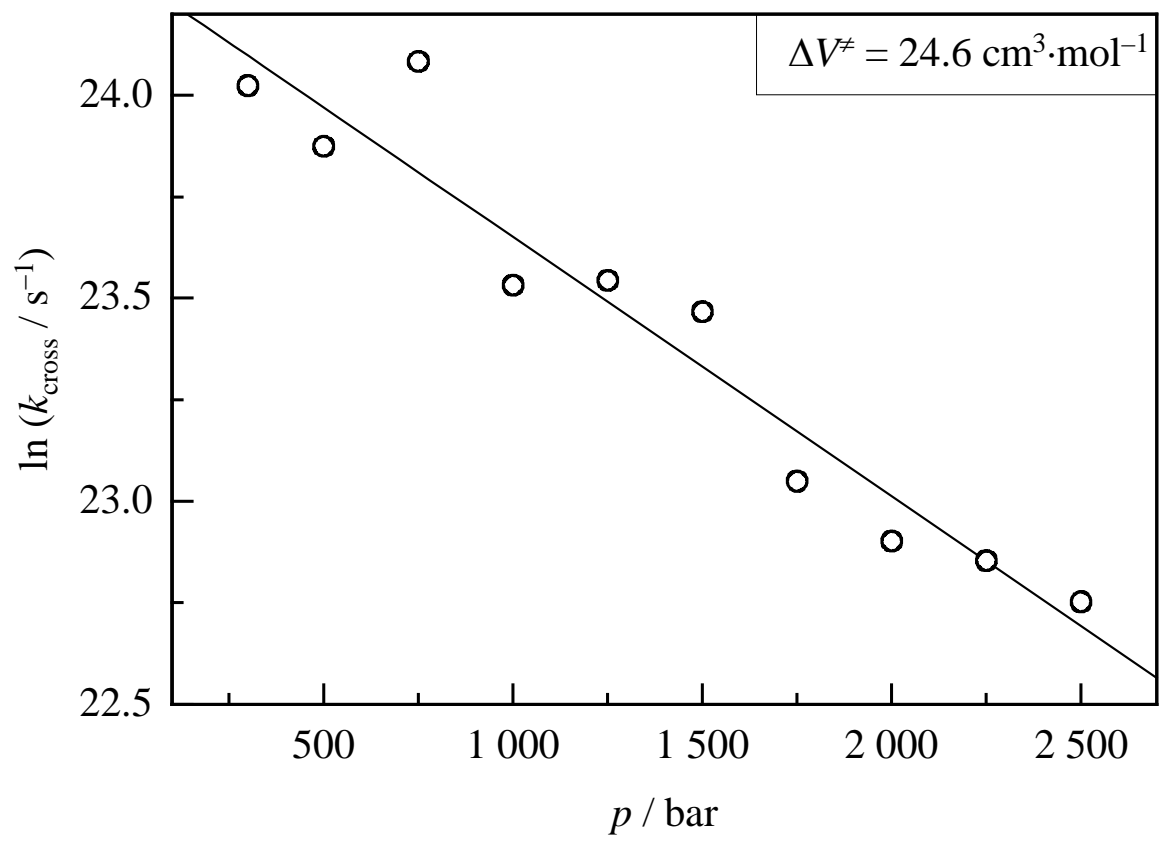

Abb. 5.11: Erhaltende Druckabhängigkeit der Geschwindigkeitskonstante für Kreuzdisproportionierung des tert-Butoxyradikals und n-Propylradikals aus Anpassung an den Stoffmengenanteil von tert-Butanol bei $190{ }^{\circ} \mathrm{C}$. $k_{\text {comb }}$ und $k_{\text {ol }}$ von TBPA übernommen. 


\subsubsection{Temperatur- und Druckabhängigkeit der Initiatoreffektivität abgeschätzt aus dem Stoffmengenanteil der Käfigprodukte für tert-Butylperoxy-n- butyrat}

In Abbildung 5.12 ist die Temperaturabhängigkeit der aus dem Stoffmengenanteil der modellierten Käfigprodukte Ether und Alken berechneten Initiatoreffektivität von TBPnB dargestellt. Diese ist wie bei TBPA temperaturunabhängig und hat ein Wert von 0.70.

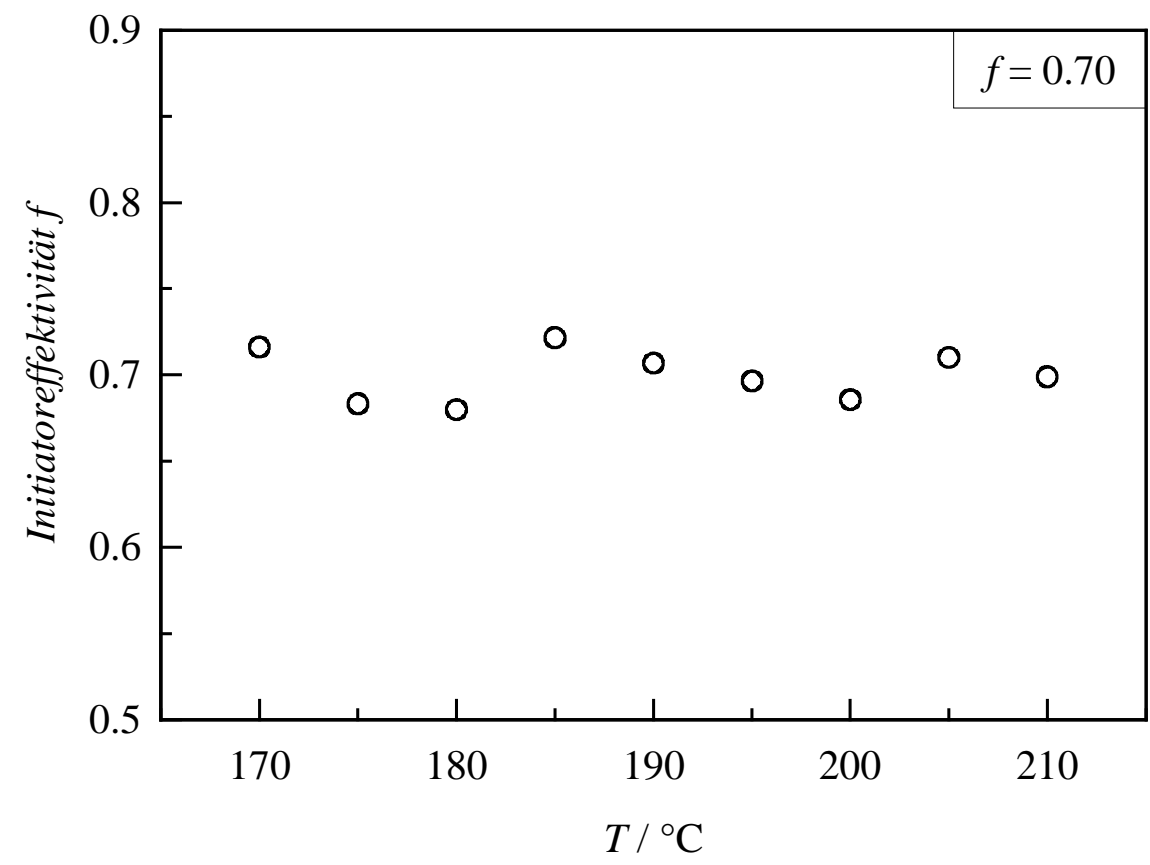

Abb. 5.12: Temperaturabhängigkeit der Initiatoreffektivität von TBPnB berechnet aus $x_{E t h e r}$ und $x_{\text {Alken }}$ der modellierten Käfigprodukte bei 500 bar.

In Abbildung 5.13 ist die Druckabhängigkeit der aus dem Stoffmengenanteil der modellierten Käfigprodukte Ether und Alken berechneten Initiatoreffektivität von TBPnB gezeigt. Die Effektivität sinkt mit dem Druck von 0.75 bei 300 bar auf 0.54 bei 2500 bar. Die berechnete Effektivität bei 2000 bar $\left(f_{\text {Käfigprodukte }}=0.59\right)$ ist viel kleiner als der Wert aus der EthenHochdruckpolymerisation für TBPPent $\left(f_{\text {Ethen }}=0.85\right)$. TBPPent und TBPnB sollten die gleiche Initiatoreffektivität besitzen. 


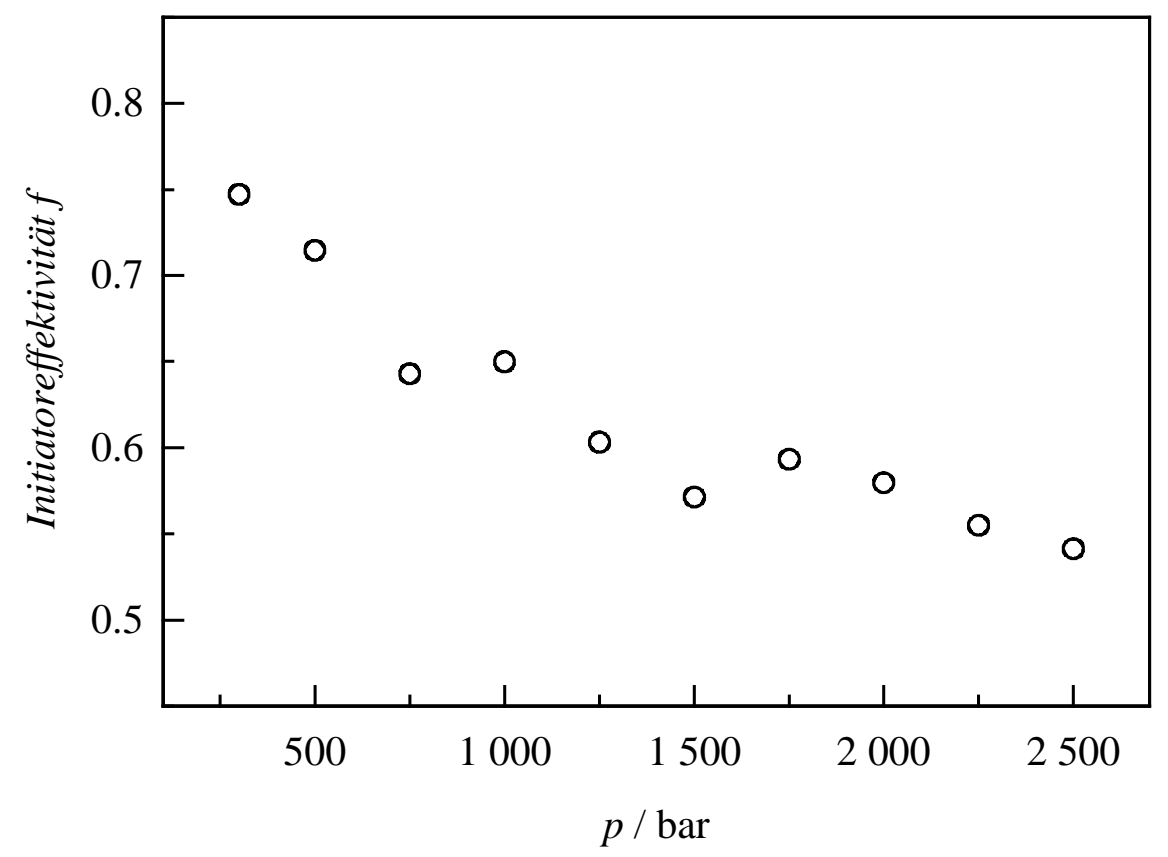

Abb. 5.13: Druckabhängigkeit der Initiatoreffektivität von TBPnB berechnet aus $x_{E t h e r}$ und $x_{\text {Alken }}$ der modellierten Käfigprodukte bei $190^{\circ} \mathrm{C}$.

\subsubsection{Modellierung der Temperatur- und Druckabhängigkeit der} Geschwindigkeitskonstanten für die Kreuzdisproportionierung des tert-Butoxy- und iso-Propylradikals

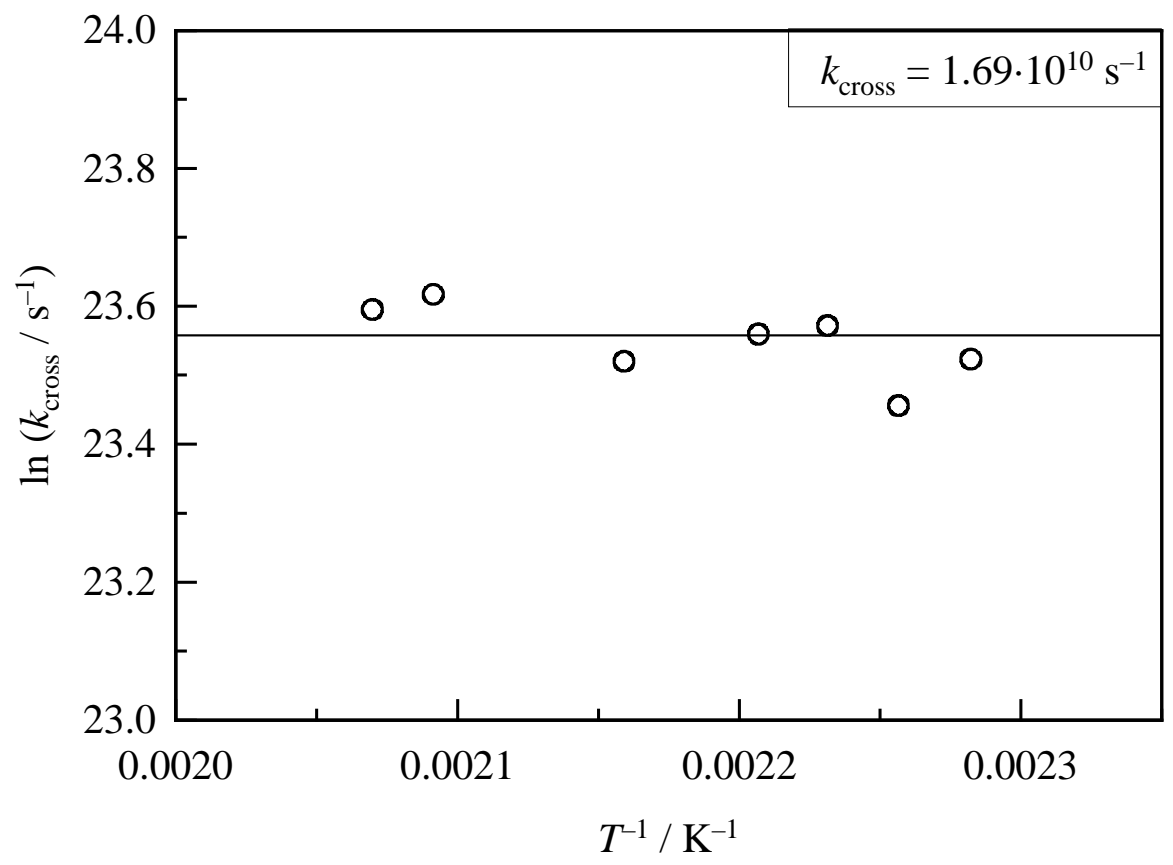

Abb. 5.14: Erhaltende Temperaturabhängigkeit der Geschwindigkeitskonstante für die Kreuzdisproportionierung des tert-Butoxyradikals und iso-Propylradikals aus Anpassung an den Stoffmengenanteil von tert-Butanol bei 500 bar. $k_{\text {comb }}$ und $k_{o l}$ von TBPA übernommen. 
Die Geschwindigkeitskonstante $k_{\text {cross }}$ wurde durch Anpassung an den Stoffmengenanteil des überschüssigen tert-Butanols erhalten. $k_{\text {comb }}$ und $k_{\mathrm{ol}}$ wurde von TBPA übernommen. Die temperaturunabhängige Geschwindigkeitskonstante der Kreuzdisproportionierung für das tert-Butoxyradikal mit dem iso-Propylradikal ist in Abbildung $5.14 \mathrm{zu}$ sehen. $k_{\text {cross }}$ sollte mit der Anzahl $\beta$-ständigen H-Atom im Alkylradikal ansteigen. Überraschenderweise ist der Wert kleiner als $k_{\text {cross }}$ für TBPnB.

In Abbildung 5.15 ist die Druckabhängigkeit der Geschwindigkeitskonstante für die Kreuzdisproportionierung des tert-Butoxyradikals mit dem iso-Propylradikal gezeigt. Das Aktivierungsvolumen $\Delta V^{\neq}$ist $15.4 \mathrm{~cm}^{3} \cdot \mathrm{mol}^{-1}$.

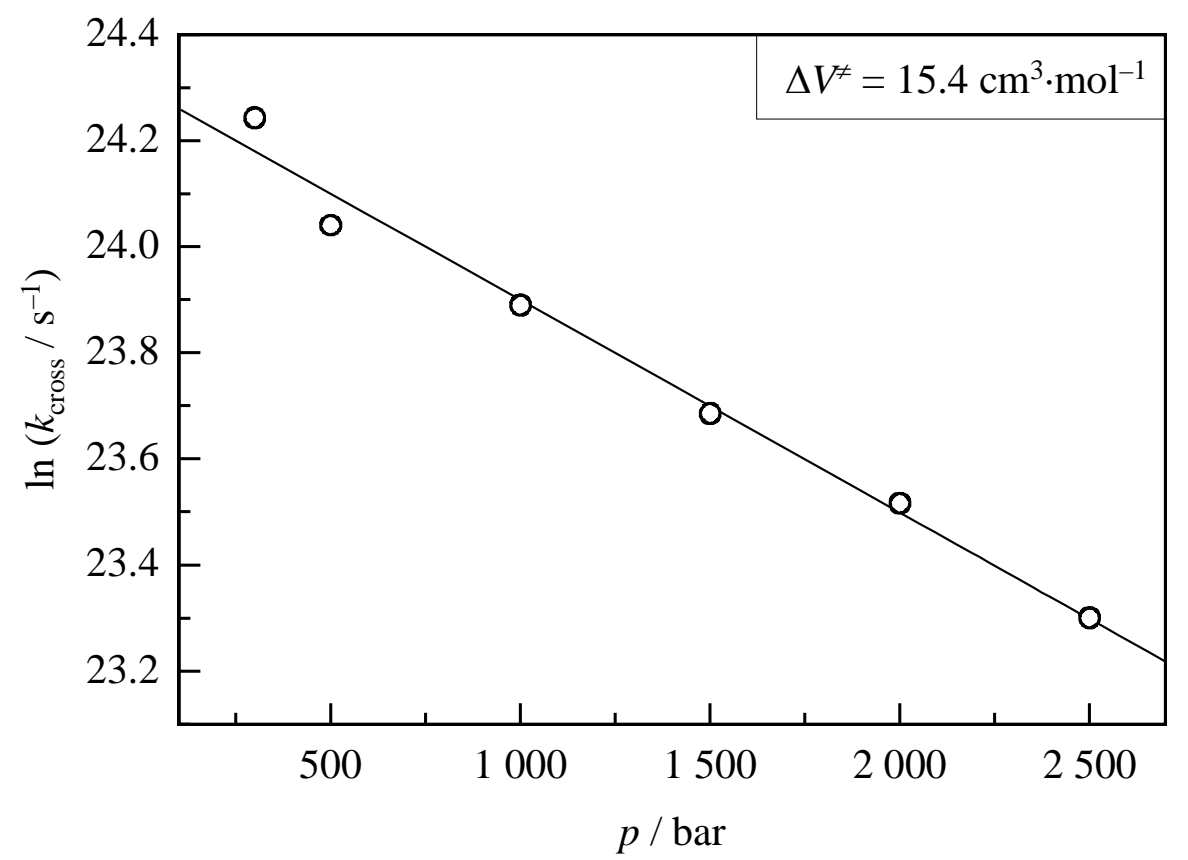

Abb. 5.15: Erhaltende Druckabhängigkeit der Geschwindigkeitskonstante für die Kreuzdisproportionierung des tert-Butoxyradikals und iso-Propylradikals aus Anpassung an den Stoffmengenanteil von tert-Butanol bei $190{ }^{\circ} \mathrm{C}$. $k_{\text {comb }}$ und $k_{o l}$ von TBPA übernommen.

\subsubsection{Temperatur- und Druckabhängigkeit der Initiatoreffektivität abgeschätzt aus dem Stoffmengenanteil der Käfigprodukte für tert-Butylperoxy-iso- butyrat}

Die Temperaturabhängigkeit der Initiatoreffektivität für TBPiB, berechnet aus dem Stoffmengenanteil der modellierten Käfigprodukte Ether und Alken, ist in Abbildung $5.16 \mathrm{zu}$ sehen. Es wird wie für die anderen bereits vorgestellten tert-Butylperoxyester eine temperaturunabhängige Initiatoreffektivität erhalten. 


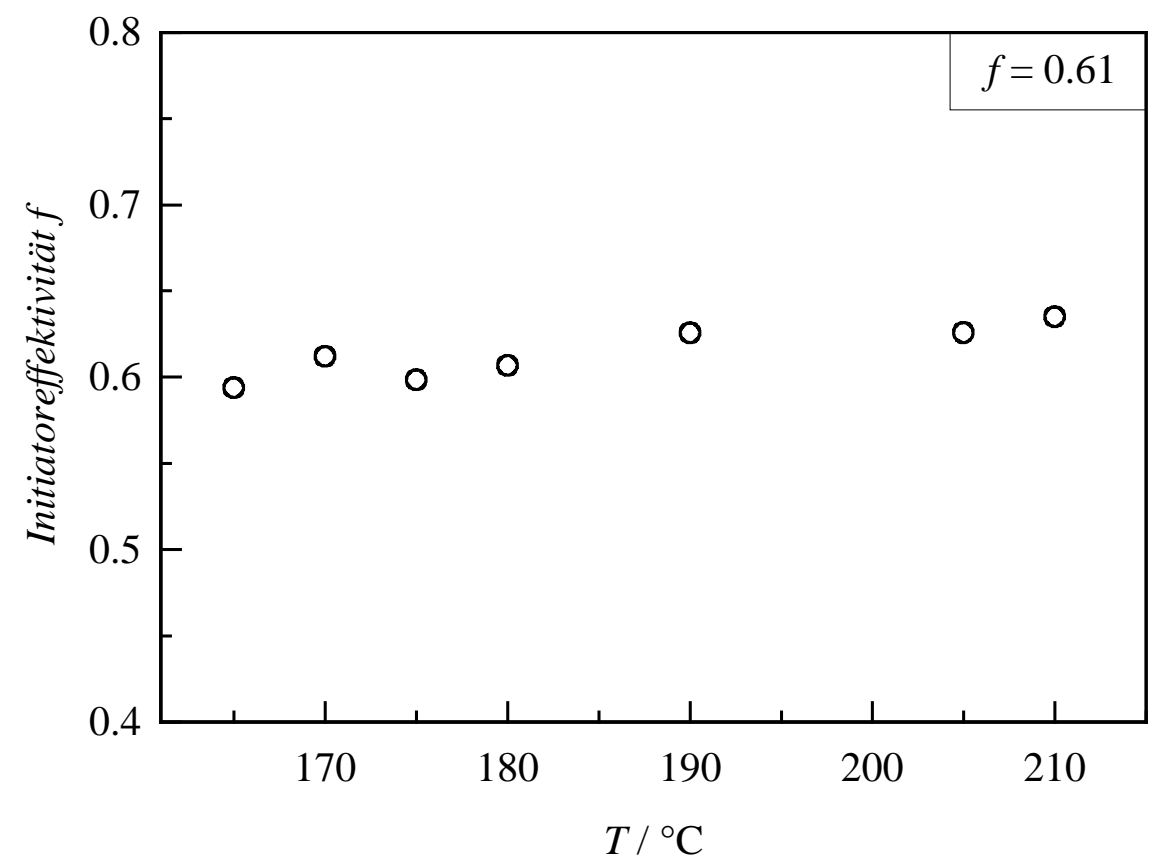

Abb. 5.16: Temperaturabhängigkeit der Initiatoreffektivität von TBPiB berechnet aus $x_{E t h e r}$ und $x_{\text {Alken }}$ der modellierten Käfigprodukte bei 500 bar.

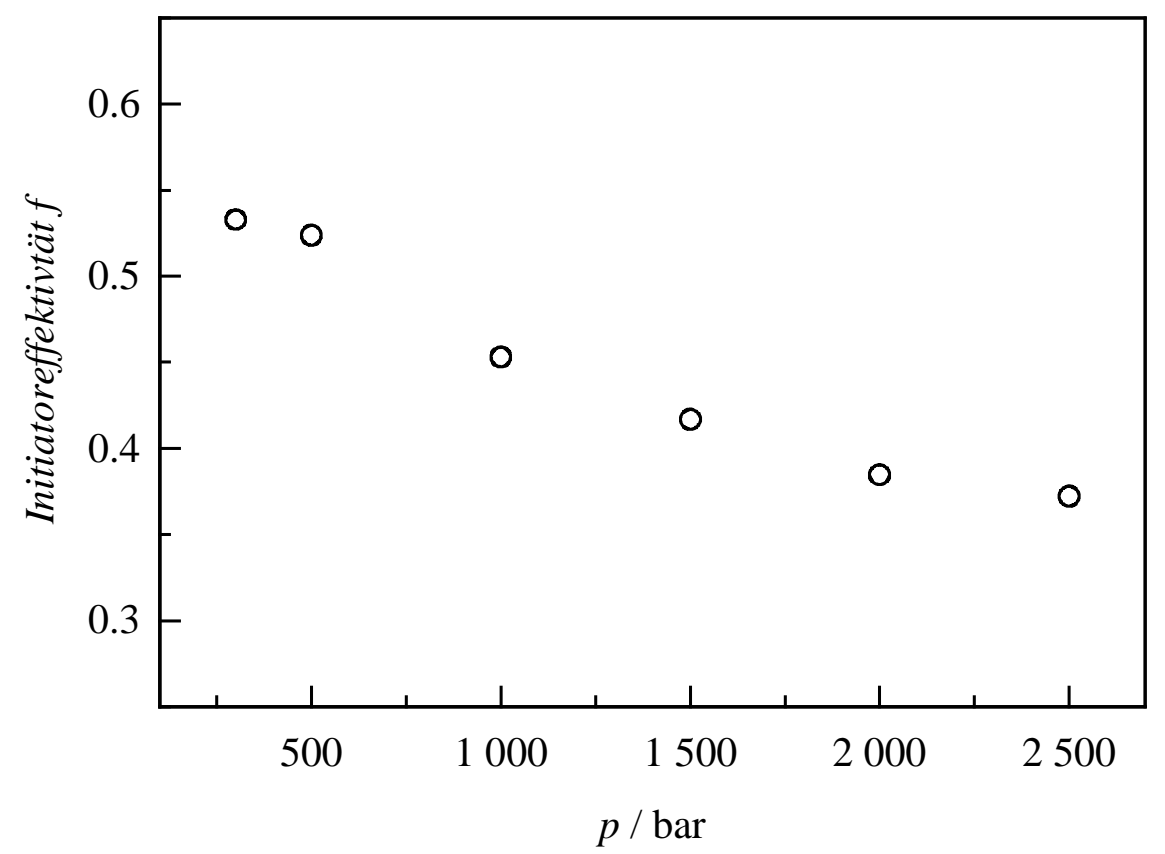

Abb. 5.17: Druckabhängigkeit der Initiatoreffektivität von TBPiB berechnet aus $x_{E t h e r}$ und $x_{\text {Alken }}$ der modellierte Käfigprodukten bei $190^{\circ} \mathrm{C}$.

In Abbildung 5.17 ist die Druckabhängigkeit der aus dem Stoffmengenanteil der modellierten Käfigprodukte Ether und Alken berechneten Initiatoreffektivität von TBPiB dargestellt. Die Effektivität nimmt ab von 0.53 bei 300 bar auf 0.37 bei 2500 bar. Die Initiatoreffektivität bei 2000 bar ist, wie auch bei TBPnB gesehen, mit $f_{\text {Käfigprodukte }}=0.39$ viel kleiner als der Wert aus der Ethen-Hochdruckpolymerisation $\left(f_{\text {Ethen }}=0.66\right)$. 


\subsubsection{Modellierung der Temperatur- und Druckabhängigkeit der Geschwindigkeitskonstanten für die Kreuzdisproportionierung des tert-Butoxy- und tert-Butylradikals}

Die Temperaturabhängigkeit der Geschwindigkeitskonstante der Kreuzdisproportionierung für das tert-Butoxyradikal mit dem tert-Butylradikal ist in Abbildung 5.18 gezeigt. $k_{\text {cross }}$ wurde durch Anpassung an den Stoffmengenanteil des überschüssigen tert-Butanols erhalten. $k_{\text {comb }}$ und $k_{\mathrm{ol}}$ wurde von TBPA übernommen. Es ist keine klare Temperaturabhängigkeit zu erkennen. Da aber die bisherigen Geschwindigkeitskonstanten der Käfigreaktionen jeweils temperaturunabhängig waren, wird für die Temperaturabhängigkeit der Kreuzdisproportionierung des tert-Butoxyradikals mit dem tert-Butylradikal ebenfalls ein konstantes $k_{\text {cross }}$ angenommen. $k_{\mathrm{cross}}$ ist wie bei TBPnB und TBPiB sehr hoch.

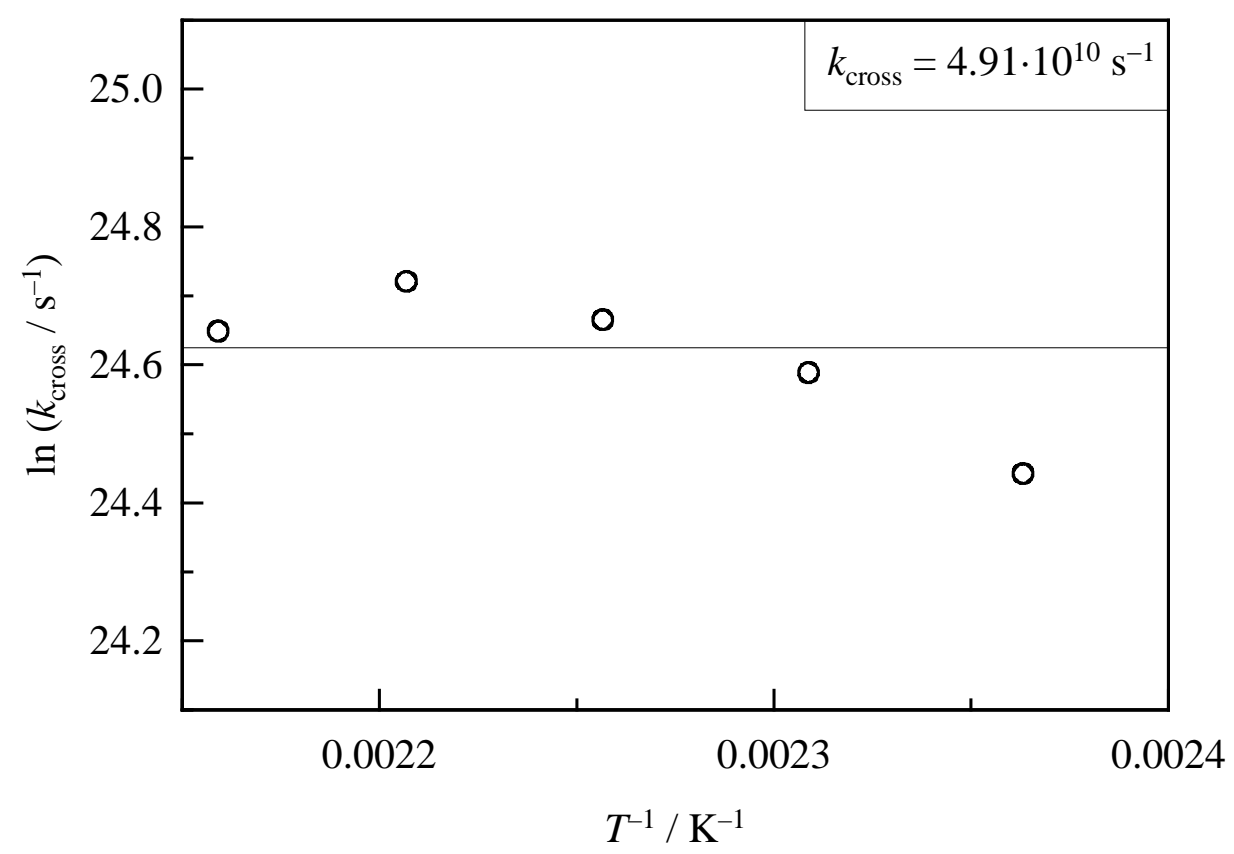

Abb. 5.18: Erhaltende Temperaturabhängigkeit der Geschwindigkeitskonstante für die Kreuzdisproportionierung des tert-Butoxyradikals und tert-Butylradikals aus Anpassung an den Stoffmengenanteil von tert-Butanol bei 500 bar. $k_{\text {comb }}$ und $k_{o l}$ von TBPA übernommen.

In Abbildung 5.19 ist die Druckabhängigkeit der Geschwindigkeitskonstante für die Kreuzdisproportionierung des tert-Butoxyradikals mit dem tert-Butylradikal dargestellt. Das Aktivierungsvolumen dieser Reaktion ist mit $4.6 \mathrm{~cm}^{3} \cdot \mathrm{mol}^{-1}$ sehr viel kleiner als die Werte der bereits untersuchten Käfigreaktionen. 


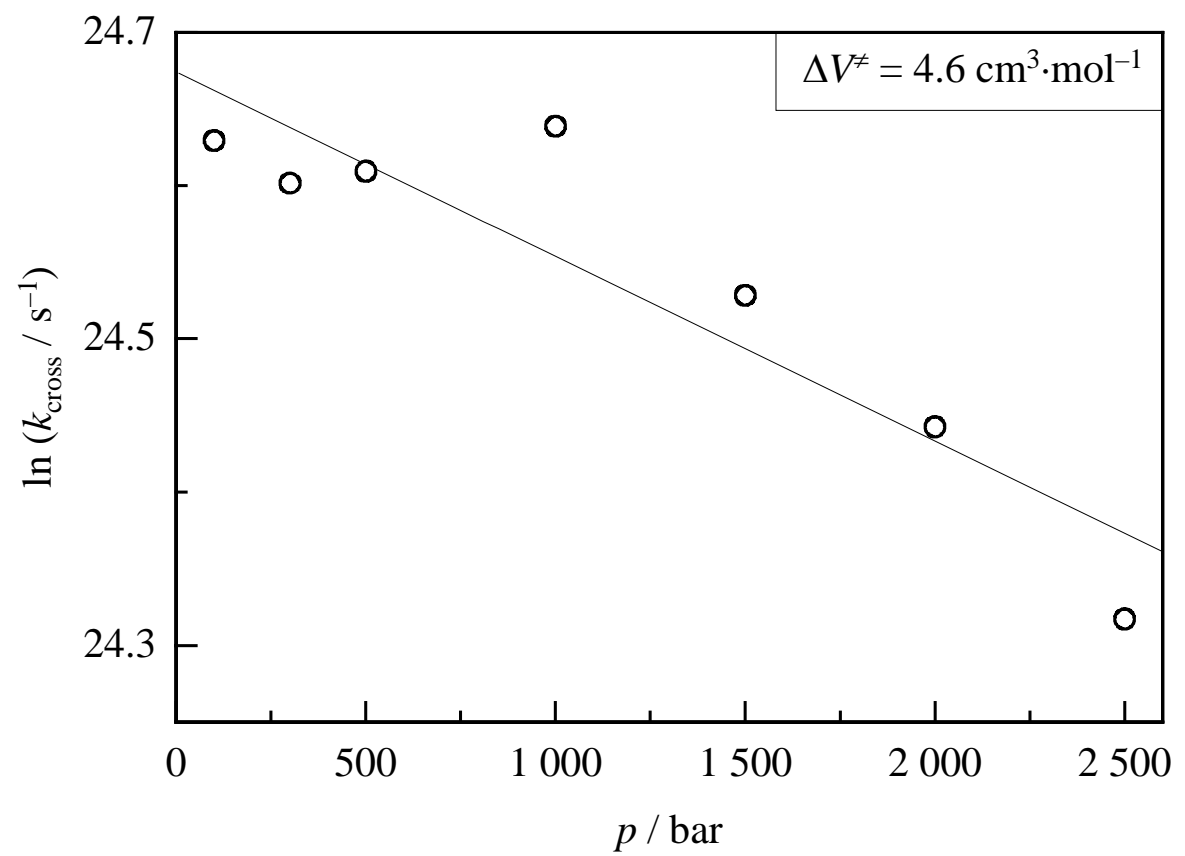

Abb. 5.19: Erhaltende Druckabhängigkeit der Geschwindigkeitskonstante für die Kreuzdisproportionierung des tert-Butoxyradikals und tert-Butylradikals aus Anpassung an den Stoffmengenanteil von tert-Butanol bei $190^{\circ} \mathrm{C}$. $k_{\text {comb }}$ und $k_{o l}$ von TBPA übernommen.

\subsubsection{Temperatur- und Druckabhängigkeit der Initiatoreffektivität abgeschätzt aus dem Stoffmengenanteil der Käfigprodukte für tert-Butylperoxypivalat}

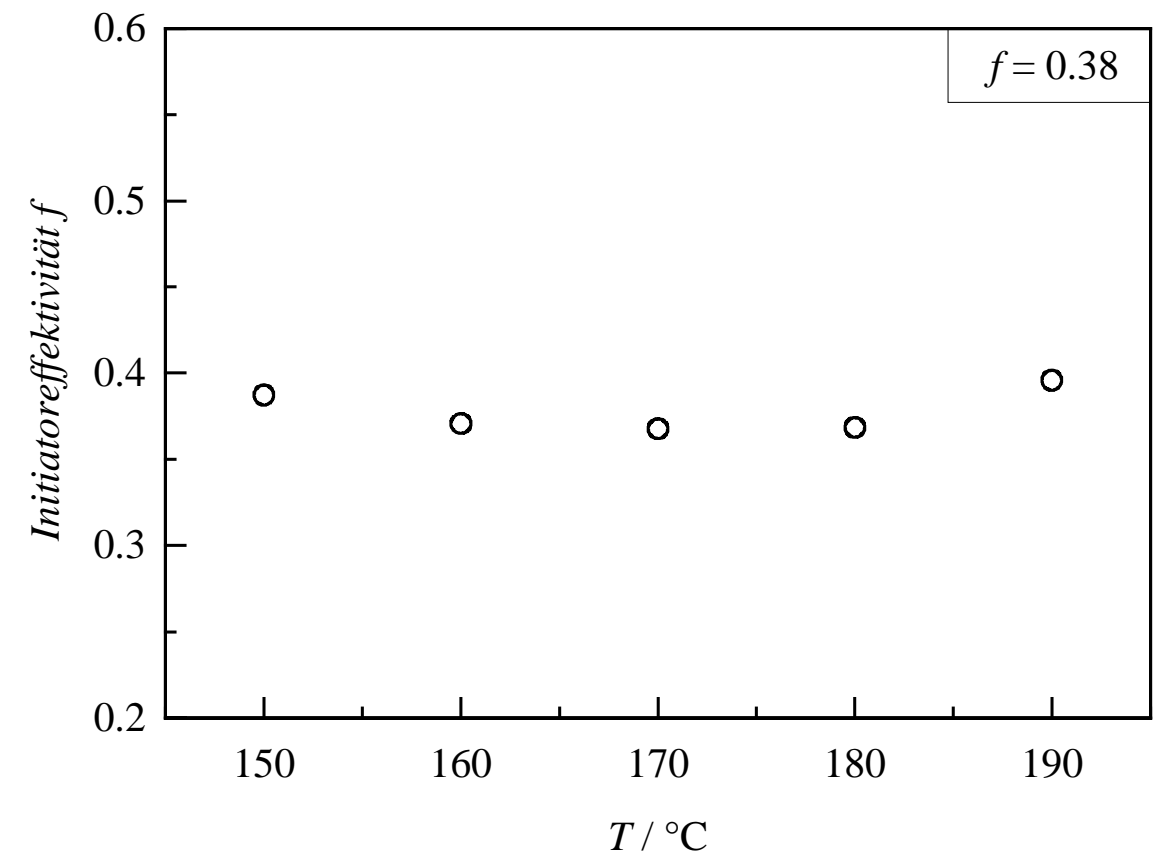

Abb. 5.20: Temperaturabhängigkeit der Initiatoreffektivität von TBPP berechnet aus $x_{\text {Ether }}$ und $x_{\text {Alken }}$ der modellierte Käfigprodukten bei 500 bar. 
Die Temperaturabhängigkeit der aus dem Stoffmengenanteil der modellierten Käfigprodukte Ether und Alken berechnete Initiatoreffektivität ist in Abbildung $5.20 \mathrm{zu}$ sehen. Es wird eine konstante Effektivität von 0.38 erhalten.

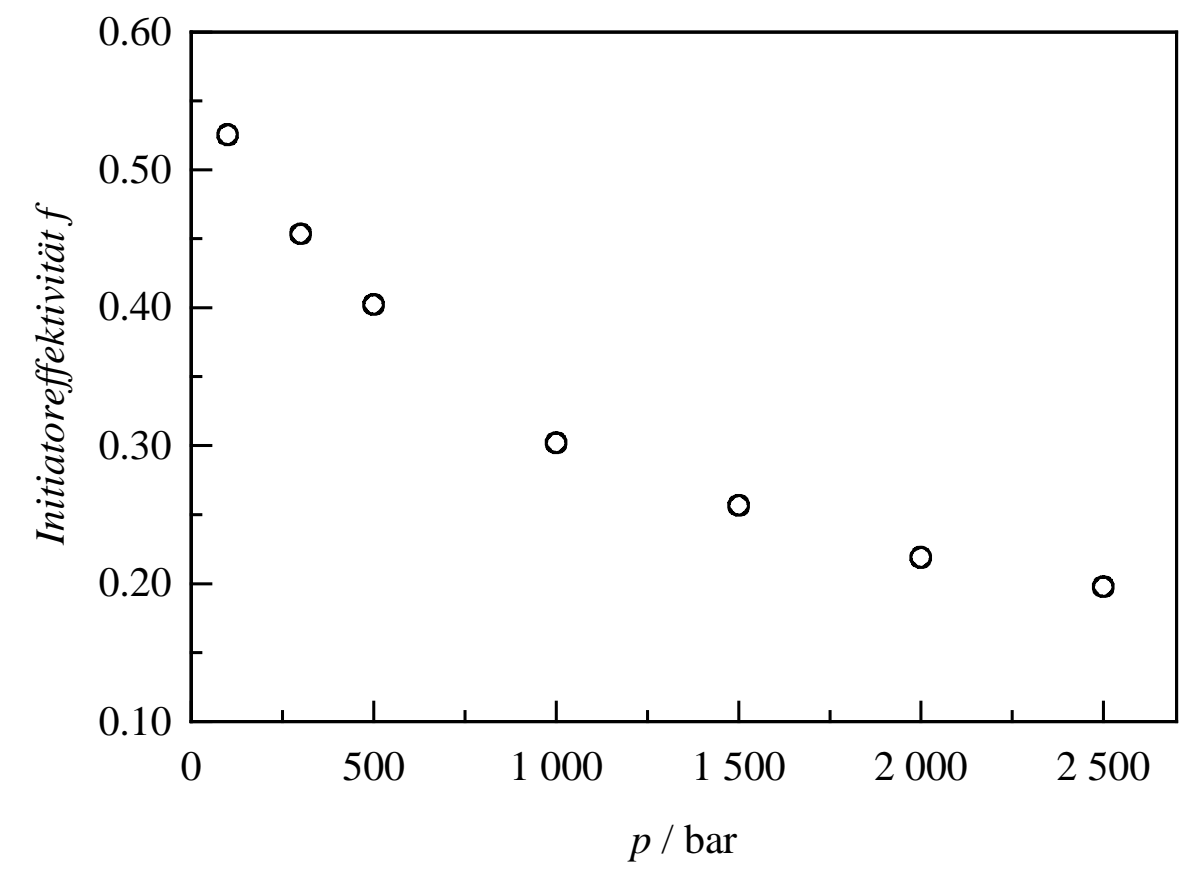

Abb. 5.21: Druckabhängigkeit der Initiatoreffektivität von TBPP berechnet aus $x_{E t h e r}$ und $x_{\text {Alken }}$ der modellierte Käfigprodukten bei $190^{\circ} \mathrm{C}$.

Die aus dem Stoffmengenanteil der modellierten Käfigprodukte Ether und Alken berechnete Initiatoreffektivität von TBPP nimmt mit dem Druck von 0.53 bei 100 bar auf 0.20 bei 2500 bar ab, wie in Abbildung 5.21 gezeigt. Dies ist die stärkste Druckabhängigkeit von den vier untersuchten tert-Butylperoxyester. Der $f$-Wert bei 2000 bar ist wie bei TBPnB und TBPiB kleiner als die gemessene Initiatoreffektivität in der Ethen-Hochdruckpolymerisation.

\subsection{Diskussion}

In diesem Abschnitt sollen die an tert-Butanol und tert-Butylmethylether angepassten Geschwindigkeitskonstanten und die aus dem Stoffmengenanteil der modellierten Käfigprodukte Ether und Alken berechneten Initiatoreffektivitäten diskutiert und mit vorhandenen Literaturwerte verglichen werden. 


\subsubsection{H-Abstraktiongeschwindigkeitskonstante des tert-Butoxyradikals von n-Heptan}

In Kapitel 5.4.1 wurde die Temperaturabhängigkeit der H-Abstraktiongeschwindigkeitskonstante gezeigt. Die Aktivierungsenergie wurde zu $27.9 \mathrm{~kJ} \cdot \mathrm{mol}^{-1}$ bei 500 bar bestimmt. Auf verschwindenden Druck extrapoliert ergibt sich $E_{\mathrm{A}}(0 \mathrm{bar})=28.4 \mathrm{~kJ} \cdot \mathrm{mol}^{-1}$. Der präexponentielle Faktor $A$ hat ein Wert von $1.0 \cdot 10^{10} \mathrm{~L} \cdot \mathrm{mol}^{-1} \cdot \mathrm{s}^{-1}$. In einem Übersichtsartikel von HENDRY und Mitarbeitern ist für die H-Abstraktion des tert-Butoxyradikals von einem sekundären aliphatischen Kohlenstoffatom eine Aktivierungsenergie von $27.8 \mathrm{~kJ} \cdot \mathrm{mol}^{-1}$ bei 1 bar angegeben [21], die in sehr guter Übereinstimmung mit der oben genannten Aktivierungsenergie ist. Für den präexponentiellen Faktor ist ein Wert pro Wasserstoffatom, das an einem sekundären aliphatischen Kohlenstoffatom gebunden ist, von $1.6 \cdot 10^{9} \mathrm{~L} \cdot \mathrm{mol}^{-1} \cdot \mathrm{s}^{-1}$ angegeben. Für $n$-Heptan mit zehn sekundären Wasserstoffatomen beträgt $A=$ 1.6 $10^{10} \mathrm{~L} \cdot \mathrm{mol}^{-1} \cdot \mathrm{s}^{-1}$. Der im Rahmen dieser Arbeit bestimmte präexponentiellen Faktor liegt um $60 \%$ darunter.

Für die Druckabhängigkeit wurde ein Aktivierungsvolumen $\Delta V^{\ddagger}\left(k_{\mathrm{ol}}\right)=-9.4 \mathrm{~cm}^{3} \cdot \mathrm{mol}^{-1}$ bestimmt. In der Literatur wird die Druckabhängigkeit des Verhältnisses der Geschwindigkeitskonstanten der H-Abstraktion von $n$-Heptan und der $\beta$-scission angegeben [22]. $\Delta V^{\ddagger}\left(k_{\mathrm{ol}}\right)$ $-\Delta V^{\ddagger}\left(k_{\beta}\right)$ beträgt $-15.5 \mathrm{~cm}^{3} \cdot \mathrm{mol}^{-1}$. In Kapitel 5.3.4 wurde aus den optimierten Geometrien der DFT-Berechnungen für die $\beta$-scission des tert-Butoxyradikals das Aktivierungsvolumen mit $\Delta V^{\neq}\left(k_{\beta}\right)=2.2 \mathrm{~cm}^{3} \cdot \mathrm{mol}^{-1}$ abgeschätzt. Mit der Differenz der Aktivierungsvolumina und $\Delta V^{\neq}\left(k_{\beta}\right)$ lässt sich $\Delta V^{\neq}\left(k_{\mathrm{ol}}\right)=-13.3 \mathrm{~cm}^{3} \cdot \mathrm{mol}^{-1}$ berechnen. Dieser Wert ist gegenüber dem experimentellen Wert von $-9.4 \mathrm{~cm}^{3} \cdot \mathrm{mol}^{-1}$ um $3.9 \mathrm{~cm}^{3} \cdot \mathrm{mol}^{-1}$ zu groß. Diese Abweichung kann durch die Abschätzung eines zu kleinen Wertes von $\Delta V^{\neq}\left(k_{\beta}\right)$ verursacht worden sein oder durch die Bestimmung eines zu kleinen $\Delta V^{\ddagger}\left(k_{\mathrm{ol}}\right)$. Dennoch ist die Übereinstimmung der bestimmten Werten mit dem Literaturwert zufriedenstellend.

\subsubsection{Geschwindigkeitskonstanten der Käfigreaktionen, $\boldsymbol{k}_{\text {comb }}$ und $\boldsymbol{k}_{\text {cross }}$}

Die Geschwindigkeitskonstanten der Käfigreaktionen für die untersuchten tertButylperoxyester sind temperaturunabhängig und bewegen sich im Bereich von $10^{9}$ bis $10^{10} \mathrm{~s}^{-1}$. Dies wurde auch für die Geschwindigkeitskonstante der Kombination von Methylund Ethylradikalen gefunden [23]. Für iso-Propyl- und tert-Butylradikale wurde eine geringfügig negative Temperaturabhängigkeit gefunden, die im Rahmen der vorliegenden 
Arbeit, vermutlich auf Grund der Streuung Primärdaten, nicht beobachtet wurde. In Tabelle 5.1 sind die Geschwindigkeitskonstanten der Käfigreaktionen aufgelistet.

\begin{tabular}{cccc}
\hline \multicolumn{2}{c}{ Radikale } & $\boldsymbol{k}_{\text {comb }} / \mathbf{s}^{\mathbf{- 1}}$ & $\boldsymbol{k}_{\text {cross }} / \mathbf{s}^{\mathbf{- 1}}$ \\
\hline & Methyl & $5.94 \cdot 10^{9}$ & - \\
\multirow{2}{*}{ tert-Butoxy +} & $n$-Propyl & $5.94 \cdot 10^{9 *}$ & $2.72 \cdot 10^{10}$ \\
& iso-Propyl & $5.94 \cdot 10^{9^{*}}$ & $1.69 \cdot 10^{10}$ \\
& tert-Butyl & $5.94 \cdot 10^{9 *}$ & $4.91 \cdot 10^{10}$ \\
\hline
\end{tabular}

Tab. 5.1: Geschwindigkeitskonstanten der Kombination und Kreuzdisproportionierung für die untersuchten tert-Butylperoxyester bei 500 bar (* = Geschwindigkeitskonstante wurde von TBPA übernommen)

Es konnte nur für TBPA die temperaturunabhängige Geschwindigkeitskonstante der Kombination bestimmt werden, weil nur für TBPA das Kombinationsprodukt (tert-Butylmethylether) in der Gaschromatographie gefunden wurde. Es ist unwahrscheinlich, dass bei der Zersetzung der drei anderen Peroxyester kein Ether gebildet wurde. Dies kann damit erklärt werden, dass der Ether sich unterhalb des Lösungsmittelpeaks im Gaschromatogramm befand. Darum wurde die Geschwindigkeitskonstante der Kombination von TBPA für die drei anderen Peroxyester zur Modellierung benutzt.

Die Kreuzdisproportionierungskonstante $k_{\text {cross }}$ wurde an den Stoffmengenanteil des überschüssigen tert-Butanols angepasst, der nicht außerhalb des Käfigs gebildet wurde. Die erhaltenden Werte für $k_{\text {cross }}$ sind im Vergleich zu $k_{\text {comb }}$ sehr hoch und unterscheiden sich nicht stark von einander $\left(1.69 \cdot 10^{10}-4.91 \cdot 10^{10} \mathrm{~s}^{-1}\right)$. Dies zeigt sich auch in zu niedrigen Initiatoreffektivitäten im Vergleich $\mathrm{zu}$ den in der Hochdruckpolymerisation von Ethen bestimmten Effektivitäten (siehe Kapitel 5.5.3). Ebenfalls überraschend ist, dass für TBPiB der kleinste Wert für $k_{\text {cross }}$ erhalten wurde. $k_{\text {cross }}$ sollte in der gleichen Reihenfolge ansteigen wie die Anzahl der $\beta$-ständigen H-Atome $\left(\mathrm{H}_{\beta}\right)$ zur Radikalfunktion im n-Propyl-, iso-Propylund im tert-Butylradikal $\left(2 \mathrm{H}_{\beta}, 6 \mathrm{H}_{\beta}, 9 \mathrm{H}_{\beta}\right)$, da durch erhöhte Anzahl der $\mathrm{H}_{\beta}$ die Wahrscheinlichkeit eines reaktiven Stoßes ansteigt.

SHELDON und KOCHI haben tert-Butylperoxyester bei $30{ }^{\circ} \mathrm{C}$ und Umgebungsdruck in Dekalin photolytisch gespalten und die Zersetzungsprodukte per GC analysiert. Anhand der Zersetzungsprodukte wurden die Verhältnisse der Geschwindigkeitskonstanten für die Käfigreaktionen ( $k_{\text {cross }} / k_{\text {comb }}$ ) bestimmt [24]. In Tabelle 5.2 werden die Verhältnisse dieser Geschwindigkeitskonstanten aus der Modellierung mit entsprechenden Literaturdaten 
verglichen. Da die Geschwindigkeitskonstanten der Käfigreaktionen temperaturunabhängig sind, ist der Vergleich der Verhältnisse der Geschwindigkeitskonstanten bei verschiedenen Temperaturen zulässig.

\begin{tabular}{cccc}
\hline \multicolumn{2}{c}{ Radikale } & $\boldsymbol{k}_{\text {cross }} / \boldsymbol{k}_{\text {comb }}$ & $\boldsymbol{k}_{\text {cross }} / \boldsymbol{k}_{\text {comb }}$ [24] \\
\hline \multirow{3}{*}{ tert-Butoxy } & Ethyl/n-Propyl & 4.58 & 0.32 \\
& iso-Propyl & 2.85 & 2.4 \\
& tert-Butyl & 8.27 & 19 \\
\hline
\end{tabular}

Tab.5.2: Verhältnisse der Geschwindigkeitskonstanten $k_{\text {cross }}$ und $k_{\text {comb }}$ bei $190{ }^{\circ} \mathrm{C}$ und 500 bar. Im Vergleich dazu Literaturwerte [24] bei $30{ }^{\circ} \mathrm{C}$ und 1 bar.

${ }^{*}=$ In der Literatur wurde das tert-Butoxy- und Ethylradikal untersucht. Im Rahmen dieser Arbeit das tert-Butoxy- und n-Propylradikal. Die beiden primären Radikale sollten eine ähnliche Reaktivität besitzen.

Die Verhältnisse der Geschwindigkeitskonstanten aus der Literatur weisen eine große Abhängigkeit von der Art des Alkylradikals auf. $k_{\text {cross }} / k_{\text {comb }}$ ist für das primäre Radikal $<1$, für das sekundäre Radikal $\approx 1$ und für das tertiäre Radikal $>1$. SHELDON und KocHI gehen davon aus, dass $k_{\text {comb }}$ sich kaum oder gar nicht für die Radikalpaare ändert und nur $k_{\text {cross }}$ die Veränderung des Verhältnisses verursacht [24]. Bei den modellierten Geschwindigkeitskonstanten ist das Verhältnis hingegen stets $>1$ und variiert nur leicht. Diese starken Abweichungen zwischen Literatur- und modellierten Werten deuten daraufhin, dass die Anpassung von $k_{\text {cross }}$ fehlerhaft ist. Dies hat wiederum Auswirkungen auf die modellierten Initiatoreffektivitäten und wird dort diskutiert (siehe Kapitel 5.5.3).

Die Geschwindigkeitskonstanten der Käfigreaktionen $k_{\text {comb }}$ und $k_{\text {cross }}$ weisen eine unterschiedlich starke Druckabhängigkeit auf (siehe Tabelle 5.3). Es scheint unwahrscheinlich, dass der Einfluss des Druckes für die Käfigreaktionen unterschiedlich ist. Hinsichtlich der Druckabhängigkeit von $k_{\text {cross }}$ sind Zweifel berechtigt. Da bereits bei der Temperaturabhängigkeit schwer nachzuvollziehende Geschwindigkeitskonstanten $k_{\text {cross }}$ erhalten wurden, wird vermutet, dass die der Auswertung zu Grunde liegenden Stoffmengenanteile an tert-Butanol für die kinetische Analyse und Modellierung nicht genau genug sind. 


\begin{tabular}{|c|c|c|c|}
\hline \multicolumn{2}{|c|}{ Radikale } & $\begin{array}{c}\Delta V^{\ddagger}\left(k_{\text {comb }}\right) / / \\
\mathbf{c m}^{3} \cdot \mathbf{m o l}^{-1}\end{array}$ & $\begin{array}{c}\Delta V^{ \pm}\left(k_{\text {cross }}\right) / \\
\mathbf{c m}^{3} \cdot \mathbf{m o l}^{-1} \\
\end{array}$ \\
\hline \multirow{4}{*}{ tert-Butoxy } & Methyl & 12.7 & - \\
\hline & n-Propyl & - & 24.6 \\
\hline & iso-Propyl & - & 15.4 \\
\hline & tert-Butyl & - & 4.6 \\
\hline
\end{tabular}

Tab. 5.3: Aktivierungsvolumina der Geschwindigkeitskonstanten für die Kombination und Kreuzdisproportionierung der untersuchten tert-Butylperoxyester bei $190{ }^{\circ} \mathrm{C}$.

\subsubsection{Die an Zerfallsprodukten modellierten Initiatoreffektivitäten der tert-Butylperoxyester}

Im Folgenden sind die Initiatoreffektivitäten, die auf der Basis des kinetischen Modells erhaltenden Stoffmengenanteile der Käfigprodukte berechnet wurden, den in der EthenHochdruckpolymerisation bei 144 bis $212^{\circ} \mathrm{C}$ und 2000 bar wirklichen gemessenen Effektivitäten verglichen (siehe Abbildung 5.22).

In Kapitel 5.4 wurde gezeigt, dass die modellierten Effektivitäten bei 500 bar temperaturunabhängig sind. Um die Effektivitäten mit den bei 2000 bar in der Ethen-Hochdruckpolymerisation experimentell bestimmten Initiatoreffektivitäten vergleichen zu können, wurde der für 2000 bar modellierte Wert verwendet, symbolisiert durch die Linien in Abbildung 5.22. Die einzelnen Punkte sind die experimentell gemessenen $f$-Werte. Nur für TBPA stimmen die experimentellen und modellierten Daten überein. Die modellierten Werte für TBPnB (vergleichbar mit TBPPent), TBPiB und TBPP sind im Mittel um 0.2 kleiner als die experimentellen Werte [1, 2, 3]. Es scheint, dass bei TBPA $k_{\mathrm{ol}} \mathrm{zu}$ klein und deswegen bei TBPnB, TBPiB und TBPP $k_{\text {cross }} z u$ groß bestimmt wurde. Dies hat zur Folge, dass für diese drei Peroxyester fälschlicherweise zu viel Käfigprodukte modelliert wurden, die die Effektivität herabsetzen. 


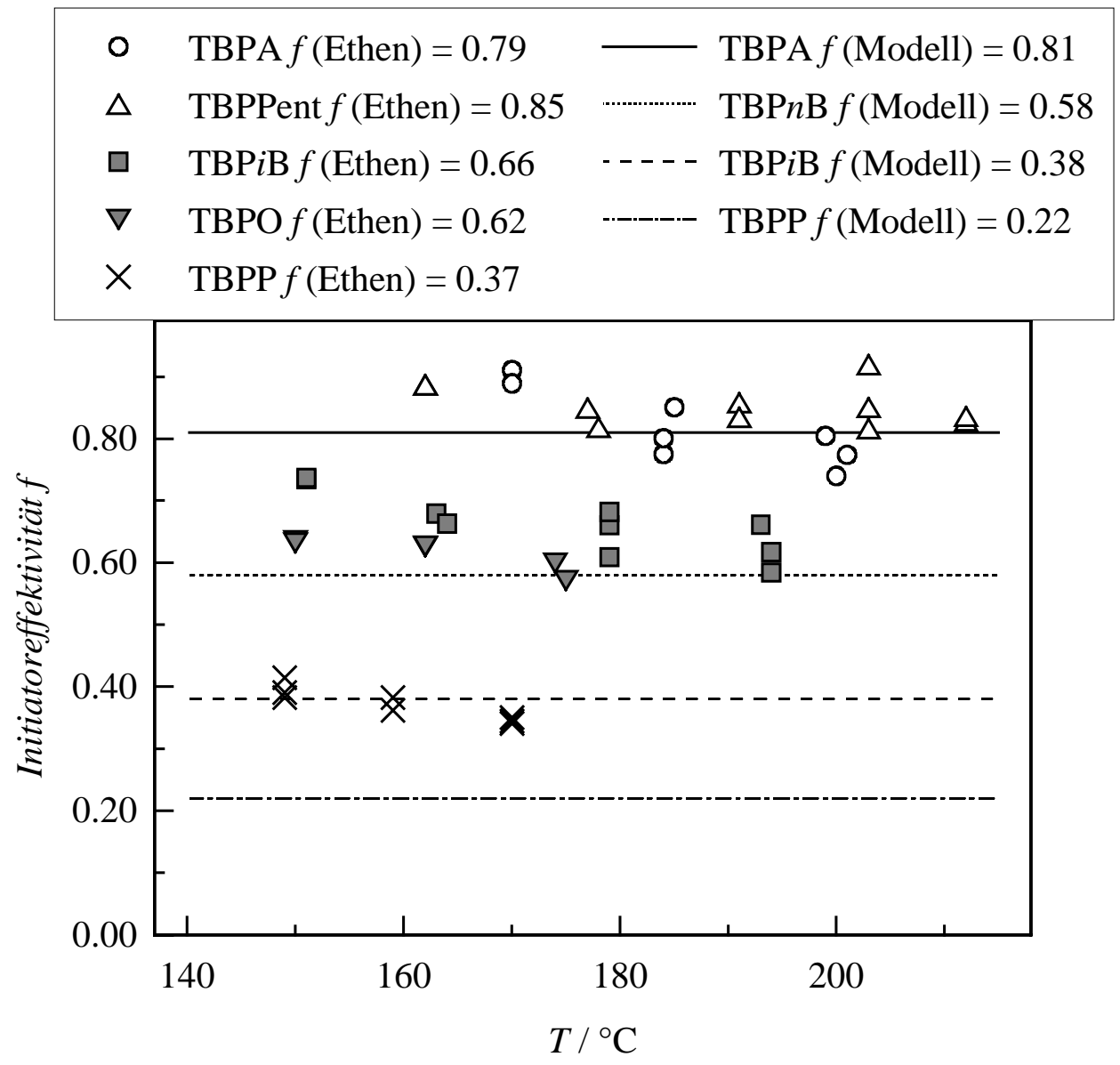

Abb. 5.22: Modellierte Initiatoreffektivitäten bei 2000 bar (Linien). Die Datenpunkte geben die experimentell in der Ethen-Hochdruckpolymerisation bestimmten Effektivitäten bei 2000 bar an [1, 2, 3].

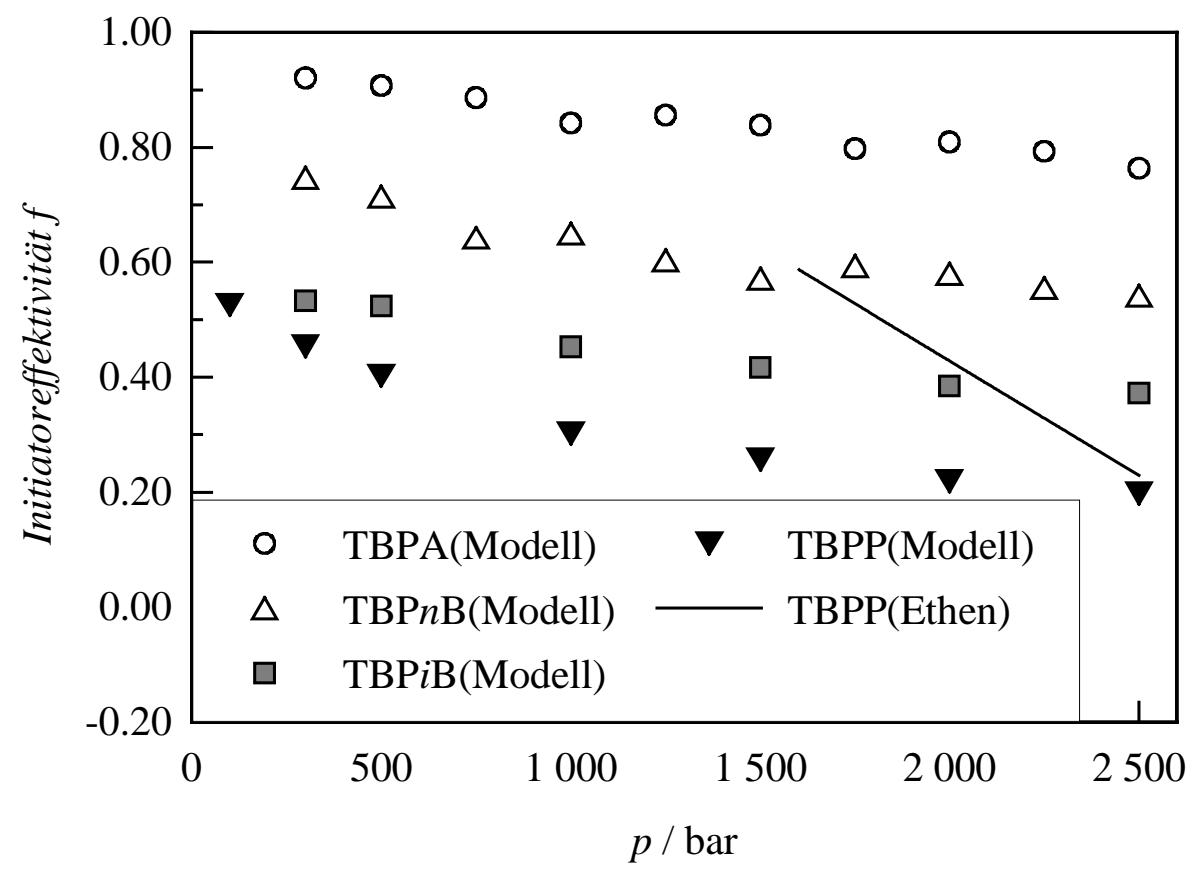

Abb. 5.23: Modellierte Initiatoreffektivitäten bei $190{ }^{\circ} \mathrm{C}$ (Punkte). Die Linie gibt die experimentell in der Ethen-Hochdruckpolymerisation bestimmten Effektivitäten für TBPP. 
In Abbildung 5.23 ist die Druckabhängigkeit der aus Stoffmengenanteile der Käfigprodukte berechneten Initiatoreffektivitäten für die untersuchten tert-Butylperoxyester zu sehen. Im Vergleich dazu ist die Druckabhängigkeit der in der Ethen-Hochdruckpolymerisation bestimmten Effektivität von TBPP dargestellt. Die modellierten Effektivitäten weisen für die untersuchten Peroxyester eine gleichartige Absenkung von $f$ mit steigendem Druck auf. Im Mittel wird die modellierte Initiatoreffektivität bei Erhöhung des Drucks von 300 auf 2500 bar um 0.2 herabgesenkt. Bei der experimentell bestimmten Initiatoreffektivität hingegen ist die Druckabhängigkeit viel stärker ausgeprägt. $f$ sinkt bei einer Drucksteigerung von 1600 auf 2500 bar um 0.35 (siehe auch Kapitel 4.10). Die Modellierung der Initiatoreffektivität unterschätzt die Druckabhängigkeit. Dies kann mit der näherungsweisen Abschätzung der Aktivierungsvolumina für die mit DFT-Berechnungen erhaltenden Geschwindigkeitskonstanten für $k_{\beta}$ und $k_{\mathrm{CO} 2}$ begründet sein. Die abgeschätzten Aktivierungsvolumina sind sehr klein. Für diese Modellierungen ist sehr wichtig, wie schnell die H-Abstraktionsreaktion des tert-Butoxyradikals außerhalb des Käfigs erfolgt, bzw. wie groß $k_{\mathrm{ol}}$ ist. Bei der Temperaturabhängigkeit der modellierten Initiatoreffektivität (Abbildung 5.22) wurde bereits gezeigt, dass $f$ für TBPnB, TBPiB und TBPP überschätzt wurde. Es scheint, dass bei der Druckabhängigkeit der modellierten Initiatoreffektivität $k_{\mathrm{ol}}$ unterschätzt ist. Wie bei der Temperaturabhängigkeit der modellierten Initiatoreffektivität ist die qualitative Aussage des Modells bei der Druckabhängigkeit von $f$ - die Effektivität sinkt mit steigendem Druck korrekt. Doch quantitativ kann das Modell die Effektivität nicht genau vorhersagen.

\subsubsection{Zusammenfassung der Diskussion}

Es wurden durch die Anpassung des Stoffmengenanteils des tert-Butanols von TBPA Geschwindigkeitskonstanten für die H-Abstraktionsreaktion des tert-Butoxyradikals mit $n$-Heptan erhalten.

Die erhaltenden Geschwindigkeitskonstanten der Käfigreaktionen, $k_{\text {comb }}$ und $k_{\text {cross, }}$ sind temperaturunabhängig. Dies oder eine sehr geringe (negative) Temperaturabhängigkeit wurde ebenfalls in der Literatur für Radikal-Radikalreaktionen in der Gasphase gefunden.

Die Initiatoreffektivitäten werden auf Grund der bisherigen Modellierung nur qualitativ richtig vorausgesagt (Temperatur- und Druckabhängigkeit, Reihung der Effektivitäten nach der Säureseite). Im Kapitel 6 wird versucht, einen Satz an Geschwindigkeitskonstanten 
zusammenzustellen, mit dem die Initiatoreffektivitäten einer Vielzahl von Peroxyester beschrieben werden kann, wobei die Anpassung der Geschwindigkeitskonstanten an experimentellen $f$-Werten aus der Ethen-Hochdruckpolymerisation erfolgt. 


\subsection{Literaturverzeichnis für Kapitel 5}

[1] J. Sandmann, Dissertation, Göttingen (2000)

[2] P. Becker, M. Buback, J. Sandmann, Macromol. Chem. Phys. 203 (2002) 2113

[3] S. Jauer, persönliche Mitteilung, (2001)

[4] D. Nelke, Diplomarbeit, Göttingen (1998)

[5] H. P. Vögele, unveröffentlichte Daten

[6] L. Wittkowski, Dissertation, Göttingen (1998)

[7] A. K. Doolittle, J. Chem. Eng. Data 9 (1964) 275

[8] R. C. Reid, T. K. Sherwood, The properties of Gases and Liquids, 2nd ed., Chemical Engineering Series, New York (1966)

[9] R. C. Neuman, R. J. Bussey, J. Am. Chem. Soc., 92 (1970) 2440

[10] L. Batt, S. Benson, J. Phys. Chem. 36 (1944) 722

[11] M. Buback, S. Klingbeil, J. Sandmann, M.-B. Sderra, H. P. Vögele, H. Wackerbarth, L. Wittkowski, Z. Phys. Chem., 210 (1999) 199

[12] M. Buback, J. Sandmann, Z. Phys. Chem., 214 (2000) 583

[13] W. A. Pryor, E. H. Morkved, H. T. Bickley, J. Org.Chem. 37 (1972) 1999

[14] M. Buback, M. Kling, S. Schmatz, eingereicht, (2005)

[15] S.D. Hamann, in High pressure physics and chemistry, Vol. II, ed. R.S. Bradlex, Academic Press, London (1965)

[16] A. Bondi, J. Phys. Chem. 68 (1964) 441

[17] E. D. Skakovskii, A. I. Stankevich, S. A. Lamotkin, L. Yu. Tychinskaya, S. V. Rykov, Russian Journal of General Chemistry 71 (2001) 615

[18] W. Braun, L. Rajbenbach, F. R. Eirich, J. Phys. Chem. 66 (1962) 1591

[19] J. W. Hilborn, J. A. Pincock, J. Am. Chem. Soc. 113 (1991) 2683

[20] M. Kling, S. Schmatz, persönliche Mitteilung, 2003

[21] D. G. Hendry, T. Mill, L. Piskiewicz, J. A. Howard, H. K. Eigenmann, J. Phys. Chem. Ref. Data 3 (1974) 937

[22] V. M. Zhulin, B. I. Rubinshtein, Bulletin of the Academy of Sciences of the USSR 10 (1976) 2055

[23] D. A. Parkes, P. C. Quinn, J. Chem Soc., Faraday Trans. 172 (1976) 1952

[24] R. A. Sheldon, J. K. Kochi, J. Am. Chem. Soc. 92 (1970) 5175 


\section{Kapitel 6}

\section{Modellierung der experimentell bestimmten Initiator- effektivitäten in der Ethen-Hochdruckpolymerisationen}

\subsection{Einleitung}

In Kapitel 5 wurde eine Predici-Modellierung der durch GC bestimmten Konzentrationsverhältnissen von Käfigprodukten aus Peroxid-Zerfallsexperimenten in $n$-Heptan vorgenommen. Hierbei zeigte es sich, dass die Geschwindigkeitskonstante $k_{\text {cross }}$ zu groß bestimmt ist, was vermutlich an einer unzutreffend bestimmten Alkoholkonzentration liegt. Im vorliegenden Kapitel sollen nun mit dem kinetischen Modell die gemessenen Initiatoreffektivitäten in der Ethen-Hochdruckpolymerisation erhalten werden, wobei die Geschwindigkeitskonstanten aus Kapitel 5 übernommen und $k_{\text {cross }}$ sowie $k_{\text {comb,Alkan }}$ (siehe Abbildung 6.1) angepasst werden. Bei der Modellierung wurde angenommen, dass $k_{\text {cross,Säure }}$ maximal gleich $k_{\text {cross }}$ ist, falls die Art des an der Reaktion beteiligten Alkylradikals gleich ist. Die Modellierungen erfolgten für experimentelle Initiatoreffektivitäten bei $190{ }^{\circ} \mathrm{C}$ und 2000 bar mit Ausnahme der Ethen-Polymerisationen mit den 1,1,2,2Tetramethylpropylperoxyestern als Initiatoren, die für die Experimente bei $140{ }^{\circ} \mathrm{C}$ und 2000 bar angepasst wurden.

\subsection{Das kinetische Schema}

In Abbildung 6.1 wird das kinetische Schema veranschaulicht. Die Geschwindigkeitskonstanten der Käfigreaktionen sind mit den fetten Lettern hervorgehoben. 

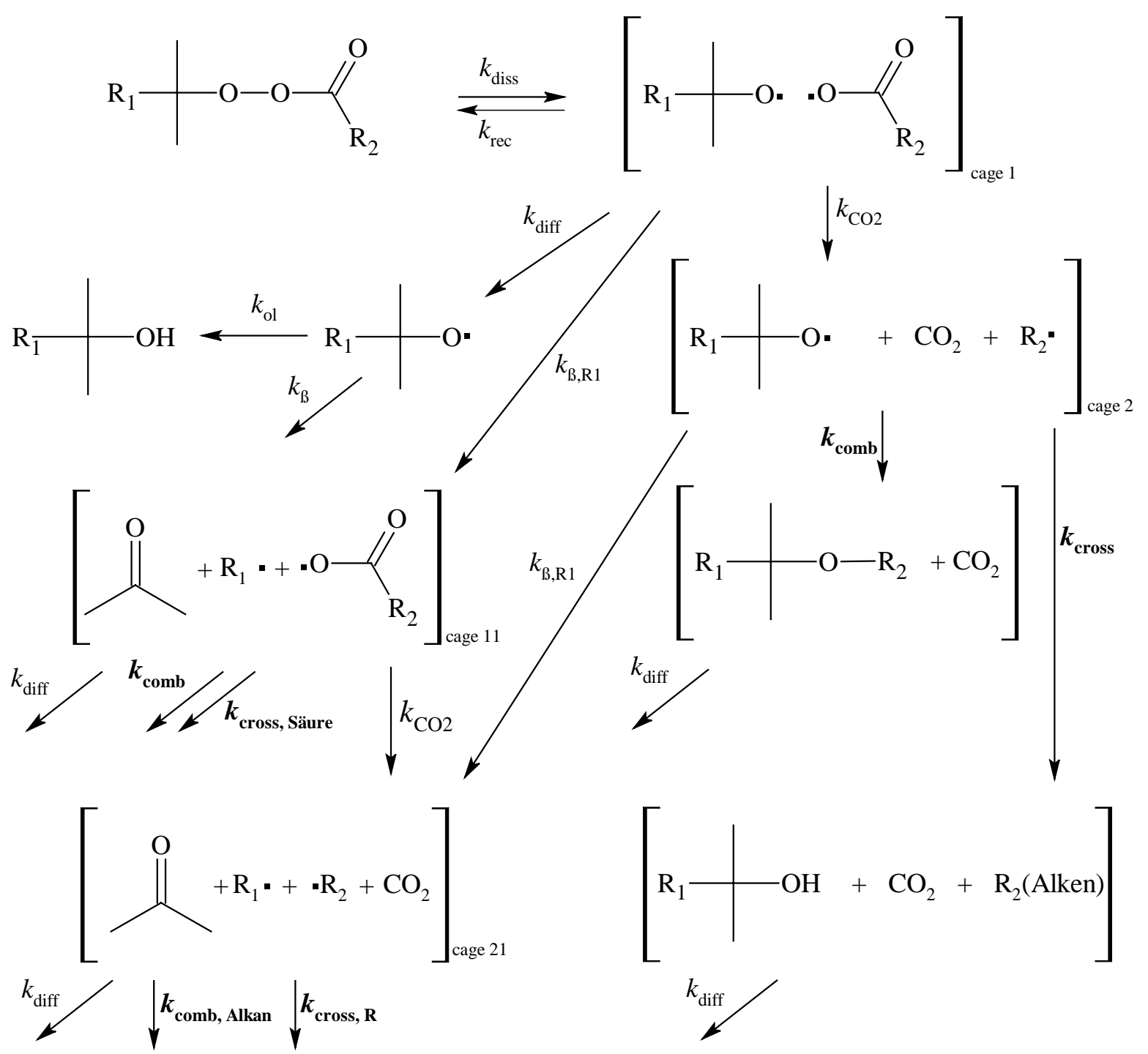

Abb. 6.1: Kinetisches Schema mit Strukturformeln. Die eckigen Klammern stellen den Lösungsmittelkäfig dar. Die Geschwindigkeitskonstanten werden im Text erklärt.

Es enthält folgende Einzelschritte:

\section{Zerfall und Rekombination}

Peroxyester $\stackrel{k_{\text {diss }}}{\longrightarrow}[\text { Alkoxyradikal }+ \text { Acyloxyradikal }]_{\text {cage } 1}$

$[\text { Alkoxyradikal }+ \text { Acyloxyradikal }]_{\text {cage } 1} \stackrel{k_{\text {rec }}}{\longrightarrow}$ Peroxyester

\section{$\underline{\text { Käfigreaktionen }}$}

$[\text { Alkoxyradikal }+ \text { Acyloxyradikal }]_{\text {cage } 1} \stackrel{k \mathrm{co} 2}{\longrightarrow}\left[\text { Alkoxyradikal }+ \text { Alkylradikal }+\mathrm{CO}_{2}\right]_{\text {cage2 }}$ 


$$
\begin{aligned}
& {[\text { Alkoxyradikal + Acyloxyradikal }]_{\text {cage } 1} \stackrel{k_{\beta, R 1}}{\longrightarrow}} \\
& {[\text { Alkylradikal + Aceton + Acyloxyradikal }]_{\text {cage11 }}} \\
& {\left[\text { Alkoxyradikal }+ \text { Alkylradikal }+\mathrm{CO}_{2}\right]_{\text {cage2 }} \stackrel{k_{\beta, \mathrm{R} 1}}{\longrightarrow}} \\
& {\left[2 \text { Alkylradikale }+ \text { Aceton }+\mathrm{CO}_{2}\right]_{\text {cage21 }}} \\
& {\left[\text { Alkoxyradikal }+ \text { Alkylradikal }+\mathrm{CO}_{2}\right]_{\text {cage2 }} \stackrel{k_{\mathrm{comb}}}{\longrightarrow} \text { Ether }+\mathrm{CO}_{2}} \\
& {\left[2 \text { Alkylradikale }+ \text { Aceton }+\mathrm{CO}_{2}\right]_{\text {cage21 }} \stackrel{k_{\text {comb }} \text { Alkan }}{\longrightarrow}\left[\text { Aceton }+\mathrm{CO}_{2}\right]_{\text {cage21,comb }}+\text { Alkan }}
\end{aligned}
$$

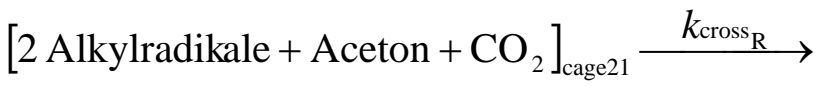

$$
\begin{aligned}
& {\left[\text { Aceton }+\mathrm{CO}_{2}\right]_{\text {cage21,cross }}+\text { Alken }_{(2)}\left(+\operatorname{Alkan}_{(2)}\right)} \\
& {[\text { Alkylradikal }+ \text { Aceton }+ \text { Acyloxyradikal }]_{\text {cage11 }} \stackrel{k_{\text {comb }}}{\longrightarrow} \text { Ester }+ \text { Aceton }} \\
& {[\text { Alkylradikal }+ \text { Aceton }+ \text { Acyloxyradikal }]_{\text {cage11 }} \stackrel{k_{\text {cross }}}{\stackrel{\text { Säure }}{\longrightarrow}}} \\
& {\left[\text { Säure }+ \text { Alken }_{(2)}\right]_{\text {cage11, cross }}+\text { Aceton }} \\
& {\left[\text { Aceton }+\mathrm{CO}_{2}\right]_{\text {cage21,comb }} \stackrel{k_{\text {fast }}}{\longrightarrow} \mathrm{CO}_{2}+\text { Aceton }} \\
& {\left[\text { Aceton }+\mathrm{CO}_{2}\right]_{\text {cage21,cross }} \stackrel{k \text { fast }}{\longrightarrow} \mathrm{CO}_{2}+\text { Aceton }} \\
& {\left[\text { Säure }+ \text { Alken }_{(2)}\right]_{\text {cage11, cross }} \stackrel{k_{\text {fast }}}{\longrightarrow} \text { Alken }_{(2)}+\text { Säure }}
\end{aligned}
$$$$
\left[\text { Alkoxyradikal }+ \text { Alkylradikal }+\mathrm{CO}_{2}\right]_{\text {cage } 2} \stackrel{k_{\text {cross }}}{\longrightarrow}\left[\text { Alken }+\mathrm{CO}_{2}\right]_{\text {cage2,cross }}+\text { Alkohol }
$$$$
\left[\text { Alken }+\mathrm{CO}_{2}\right]_{\text {cage2,cross }} \stackrel{k_{\text {fast }}}{\longrightarrow} \mathrm{CO}_{2}+\text { Alken }
$$

\section{$\underline{\text { Diffusion }}$}

$[\text { Alkoxyradikal }+ \text { Acyloxyradikal }]_{\text {cage } 1} \stackrel{k_{\text {diff }}}{\longrightarrow}$ Alkoxyradikal + Acyloxyradikal

$\left[\text { Alkoxyradikal }+ \text { Alkylradikal }+\mathrm{CO}_{2}\right]_{\text {cage2 }} \stackrel{k_{\text {diff }}}{\longrightarrow}$

$\left[\text { Alkylradikal }+\mathrm{CO}_{2}\right]_{\text {cage2,Rest }}+$ Alkoxyradikal

$\left[\text { Alkylradikal }+\mathrm{CO}_{2}\right]_{\text {cage2,Rest }} \stackrel{k \text { fast }}{\longrightarrow} \mathrm{CO}_{2}+$ Alkylradikal

$[\text { Alkylradikal }+ \text { Aceton }+ \text { Acyloxyradikal }]_{\text {cage11 }} \stackrel{k_{\text {diff }}}{\longrightarrow}$

$[\text { Alkylradikal }+ \text { Aceton }]_{\text {cage11a }}+$ Acyloxyradidal

$\left[2 \text { Alkylradikale }+ \text { Aceton }+\mathrm{CO}_{2}\right]_{\text {cage21 }} \stackrel{k_{\text {diff }}}{\longrightarrow}[2 \text { Alkylradikal }+ \text { Aceton }]_{\text {cage11a }}+\mathrm{CO}_{2}$ 


$$
[\text { Alkylradikal }+ \text { Aceton }]_{\text {cage11a }} \stackrel{k_{\text {fast }}}{\longrightarrow} \text { Aceton }+ \text { Alkylradikal }
$$

Die Reaktionsschritte mit $k_{\text {fast }}$ dienen dazu, die restlichen Käfigprodukte entstehen zu lassen, da Predici nur zwei Produktmoleküle pro Reaktion modellieren kann. Das Käfigprodukt Alkan $_{(2)}$ wurde nicht in Predici implementiert, weil die Bildung von Alken (2) ausreicht um $f$ zu berechnen.

Das kinetische Schema musste um die Reaktionsschritte mit $k_{\beta, \mathrm{R}}, k_{\text {comb,Alkan, }} k_{\text {cross,R }}$ und $k_{\text {cross,Säure }}$ erweitert werden, da bei den „Nicht-tert-Butylperoxyester“ im Kapitel 4 untersuchten tert-Alkylperoxyestern die $\beta$-scission innerhalb des Käfigs nicht ausgeschlossen werden kann. Die untersuchten tert-Alkoxyradikale, bis auf das tert-Butoxyradikal, können zusätzlich zur Methylabspaltung mit höherer Geschwindigkeit Alkylradikal abspalten.

Erfolgt die $\beta$-scission nach dem primären Bindungsbruch schneller als die Decarboxylierung, kann es zur Kombination des Alkyl- und Acylradikals zum Ester ( $k_{\text {comb; }}$ für diese Reaktion wird die gleiche Geschwindigkeitskonstante verwendet wie für die Kombination von tert-Alkoxy- und Alkylradikalen) oder zur Kreuzdisproportionierung zur Säure und zum Alken ( $\left.k_{\text {cross,Säure }}\right)$ kommen. Wenn die $\beta$-scission und die Decarboxylierung im Lösungsmittelkäfig erfolgen, können die Alkylradikale kombinieren ( $\left.k_{\text {comb,Alkan }}\right)$ oder disproportionieren $\left(k_{\mathrm{cross}, \mathrm{R}}\right)$.

Für die Selbst-Kombination von Methyl-, Ethyl-, iso-Propyl und tert-Butylradikalen sind absolute Geschwindigkeitskonstanten von PARKES und QUINN bekannt [1]. Die Geschwindigkeitskonstante der Selbst-Disproportionierung lässt sich aus den literaturbekannten Verhältnissen von $k_{\text {cross, } \mathrm{R}} / k_{\text {comb,Alkan }}$ bestimmen [2, 3, 4, 5]. Die Geschwindigkeitskonstanten für die Kreuz-Kombination lassen sich durch das geometrische Mittel der Geschwindigkeitskonstanten der Selbst-Kombination abschätzen [6]:

$$
k_{\mathrm{AB}}=2 \cdot \sqrt{k_{\mathrm{AA}} \cdot k_{\mathrm{BB}}}
$$

mit

$k_{\mathrm{AB}}=$ Geschwindigkeitskonstante der Kreuzkombination von A und $\mathrm{B}$

$k_{\mathrm{AA}}=$ Geschwindigkeitskonstante der Selbstkombination von A

$k_{\mathrm{BB}}=$ Geschwindigkeitskonstante der Selbstkombination von B

Für die Kreuz-Disproportionierung gibt es wiederum in der Literatur Daten zum Verhältnis der Geschwindigkeitskonstanten [2, 3, 4, 5, 7], so dass $k_{\text {cross }}$ mit $k_{\text {comb }}$ berechnet werden kann. Die oben genannten Geschwindigkeitskonstanten sind Werte für die bimolekularen 
Reaktionen, so dass sie nicht direkt für das Modell genutzt werden konnten (siehe Kapitel 5.2). Doch wurde der relative Gang der Geschwindigkeitskonstanten ersichtlich, an dem sich die Modellierung orientierte.

\subsection{Ergebnisse}

In diesem Abschnitt werden die verwendeten Geschwindigkeitskonstanten der Käfigreaktionen vorgestellt, die benötigt werden, um die Initiatoreffektivität der tert-Alkylperoxyester in der Ethen-Hochdruckpolymerisation zu modellieren.

\subsection{1 tert-Butylperoxyester}

Für fünf tert-Butylperoxyester (TBPA, TBPPent, TBPiB, TBPO, TBPP) ist die Initiatoreffektivität in der Ethen-Hochdruckpolymerisation bei 2000 bar bekannt (siehe Kapitel 4) $[8,9,10]$. Da die $\beta$-scission Reaktion des tert-Butoxyradikals nicht im Lösungsmittelkäfig erfolgt, können nur zwei Käfigreaktionen innerhalb der Käfiglebensdauer ablaufen (KreuzKombination mit $k_{\text {comb }}$ und Kreuzdisproportionierung mit $k_{\text {cross }}$ ). Die Geschwindigkeitskonstante $k_{\text {comb }}$ wird aus Kapitel 5 übernommen. In Predici wurde $k_{\text {cross }}$ derartig angepasst, dass die Summe der Stoffmengenanteile der Käfigprodukte $1-f_{\text {Ethen }}$ entsprach. Der Stoffmengenanteil und die Initiatoreffektivität werden wie in Gleichung 5.6 und 5.7 in Kapitel 5.4.2 berechnet. In Tabelle 6.1 sind die erhaltenden Geschwindigkeitskonstanten, Stoffmengenanteile der modellierten Käfigprodukte Ether und Alken, und die daraus resultierenden Effektivitäten $f_{\text {Ethen }}$ aufgelistet.

Die mit dem Modell bestimmte Effektivität des TBPPent kann durch den zusätzlichen Reaktionsweg nur kleiner oder gleich der Initiatoreffektivität des TBPA sein, weil bis auf $k_{\text {cross }}$ die Geschwindigkeitskonstanten für TBPA und TBPPent identisch sind. Deshalb wurde versucht, Effektivitäten zu modellieren, die zwischen $f_{\text {Ethen }}$ von TBPA und TBPPent liegen.

Die Anpassung ergibt Werte für $k_{\text {cross, }}$ die vom primären Peroxyester TBPPent über die sekundären zum tertiären Peroxyester (TBPP) deutlich ansteigen. 


\begin{tabular}{cccccc}
\hline Peroxyester & $\boldsymbol{k}_{\text {cross }} / \mathbf{s}^{-\mathbf{1}}$ & $\boldsymbol{x}_{\text {Ether }}$ & $\boldsymbol{x}_{\text {Alken }}$ & $\boldsymbol{f}_{\text {Käfigprodukte }}$ & $\boldsymbol{f}_{\text {Ethen }}[\mathbf{8 , 9}, \mathbf{9 0}]$ \\
\hline TBPA & - & 0.18 & - & 0.82 & 0.79 \\
TBPPent & $8.0 \cdot 10^{8}$ & 0.17 & 0.03 & 0.80 & 0.85 \\
TBPiB & $2.5 \cdot 10^{9}$ & 0.21 & 0.13 & 0.66 & 0.66 \\
TBPO & $4.0 \cdot 10^{9}$ & 0.20 & 0.18 & 0.62 & 0.62 \\
TBPP & $1.7 \cdot 10^{10}$ & 0.12 & 0.51 & 0.37 & 0.37 \\
\hline
\end{tabular}

Tab. 6.1: Die an die experimentell bestimmten Initiatoreffektivitäten [8, 9, 10] angepassten Kreuzdisproportionierungsgeschwindigkeitskonstanten. Stoffmengenanteile der modellierten Käfigprodukte und die mit dem Modell erhaltende Effektivitäten für $190{ }^{\circ} \mathrm{C}$ und 2000 bar.

Der Stoffmengenanteil an Ether in Tabelle 6.1 ist wegen dem konstanten $k_{\text {comb }}$ nahezu konstant für die fünf untersuchten tert-Butylperoxyester. Für die Abnahme der Initiatoreffektivität bei Variation der Säureseite ist neben der beschleunigten Decarboxylierung der Anstieg von $k_{\text {cross }}$ verantwortlich (der Stoffmengenanteil an Alken steigt von 0.03 bei TBPPent auf 0.51 bei TBPP). $k_{\text {cross }}$ steigt mit der Anzahl der $\beta$-ständigen H-Atome zur Radikalfunktion an. In Tabelle 6.2 werden die von SHELDON und KocHI und die aus der Modellierung bestimmten Verhältnisse der Geschwindigkeitskonstanten $k_{\text {cross }} / k_{\text {comb }}$ verglichen [11]. In diesem Fall ändern sich die Verhältnisse der Geschwindigkeitskonstanten gleichartig, doch sind alle modellierten Verhältnisse für $190{ }^{\circ} \mathrm{C}$ und 2000 bar kleiner als die aus der Literatur bestimmten Werte für $30^{\circ} \mathrm{C}$ und Umgebungsdruck besonders beim Radikalpaar tert-Butoxy/tert-Butyl.

\begin{tabular}{cccc}
\hline \multicolumn{1}{c}{ Radikale } & $\boldsymbol{k}_{\text {cross }} / \boldsymbol{k}_{\text {comb }}$ & $\boldsymbol{k}_{\text {cross }} / \boldsymbol{k}_{\text {comb }}$ [11] \\
\hline & Ethyl/n-Butyl & 0.20 & 0.32 \\
tert-Butoxy + & iso-Propyl & 0.62 & 2.4 \\
& 2-Heptyl & 0.99 & - \\
tert-Butyl & 4.3 & 19 \\
\hline
\end{tabular}

Tab. 6.2: Verhältnisse der aus der Modellierung entnommenen Geschwindigkeitskonstanten $k_{\text {cross }}$ und $k_{\text {comb. }}$ Im Vergleich dazu Literaturwerte [11].

* = In der Literatur wurde das tert-Butoxy- und Ethylradikal untersucht. Im Rahmen dieser Arbeit das tert-Butoxy- und n-Butylradikal. Die beiden primären Radikale sollten eine ähnliche Reaktivität besitzen. 


\subsubsection{1,1,3,3-Tetramethylbutylperoxyester}

Beim Zerfall der 1,1,3,3-Tetramethylperoxyester entsteht nach dem primären Bindungsbruch und der $\beta$-scission des 1,1,3,3-Tetramethylbutyloxyradikals ein neo-Pentylradikal. Dieses Radikal kann nicht zum Alken disproportionieren, so dass die Disproportionierung mit dem Acetoxy- $\left(k_{\text {cross,Säure }}=0\right)$ und Methylradikal $\left(k_{\text {cross,R }}=0\right.$ bei TMBPA $)$ nicht möglich ist und die Kreuzdisproportionierung mit einem Alkylradikal nur einseitig erfolgen kann. In Tabelle 6.3 und 6.4 sind die erhaltenden Geschwindigkeitskonstanten, Käfigprodukte und modellierten Effektivitäten für die 1,1,3,3-Tetramethylbutylperoxyester aufgelistet.

Anders als bei den tert-Butylperoxyestern ist die $\beta$-scission ( $k_{\beta, \mathrm{R}}$ aus DFT-Berechnungen) bei den 1,1,3,3-Tetramethylbutylperoxyester ausreichend schnell, so dass es zu Alkyl-Alkylreaktionen kommen kann.

Zunächst wurde $k_{\text {comb,Alkan }}$ für TMBPA angepasst. Durch die relativen Geschwindigkeitskonstanten aus der Literatur ergeben sich die restlichen $k_{\text {comb,Alkan }}$ und $k_{\text {cross,R }}$ für die anderen Peroxyester, so dass nur noch $k_{\text {cross }}$ angepasst wurde.

Die Kombination eines primären mit einem primären bzw. sekundären Alkylradikal erfolgt sehr schnell. Die Kombination eines primären mit einem tertiären Radikal ist bedeutend langsamer. Dies führt dazu, dass Alkan in geringen Mengen (s. Tabelle 6.4) bei TMBPA und TMBPO gebildet wird, das die Effektivität herabsetzt. Es wird ausschließlich Ether, Alken und Alkan gebildet.

\begin{tabular}{cccc}
\hline Peroxyester & $\boldsymbol{k}_{\text {cross }} / \mathbf{s}^{\mathbf{- 1}}$ & $\boldsymbol{k}_{\text {comb,Alkan }} / \mathbf{s}^{\mathbf{- 1}}$ & $\boldsymbol{k}_{\text {cross, } \mathbf{R}} / \mathbf{s}^{-\mathbf{1}}$ \\
\hline TMBPA & - & $2.0 \cdot 10^{10}$ & - \\
TMBPO & $3.5 \cdot 10^{9}$ & $1.0 \cdot 10^{10}$ & $2.0 \cdot 10^{9}$ \\
TMBPP & $1.2 \cdot 10^{10}$ & $4.0 \cdot 10^{9}$ & $2.0 \cdot 10^{9}$ \\
\hline
\end{tabular}

Tab. 6.3: Die an die im Rahmen dieser Arbeit experimentell bestimmten Initiatoreffektivitäten angepassten Kreuzdisproportionierungsgeschwindigkeitskonstanten.

\begin{tabular}{cccccc}
\hline Peroxyester & $\boldsymbol{x}_{\text {Ether }}$ & $\boldsymbol{x}_{\text {Alken }}$ & $\boldsymbol{x}_{\text {Alkan }}$ & $\boldsymbol{f}_{\text {Käfigprodukte }}$ & $\boldsymbol{f}_{\text {Ethen }}$ \\
\hline TMBPA & 0.15 & - & 0.10 & 0.75 & 0.75 \\
TMBPO & 0.17 & 0.15 & 0.07 & 0.61 & 0.61 \\
TMBPP & 0.12 & 0.36 & 0.02 & 0.50 & 0.50 \\
\hline
\end{tabular}

Tab. 6.4: Stoffmengenanteile der modellierten Käfigprodukte und die mit dem Modell erhaltenden Effektivitäten für 2000 bar. 


\subsection{3 tert-Amylperoxyester}

Für die Modellierung der Initiatoreffektivität von TAPA sind die Geschwindigkeitskonstanten für $k_{\text {comb,Alkan }}$ und $k_{\text {cross,R }}$ durch die Literaturwerte und die modellierte Geschwindigkeitskonstante $k_{\text {comb,Alkan }}$ für TMBPA bekannt. Für die Kreuzdisproportionierung des Acyloxy- und Ethylradikals wurde jeweils derselbe Wert für $k_{\mathrm{cross}, S a ̈ u r e ~}$ gewählt. Als Höchstwert wurde der

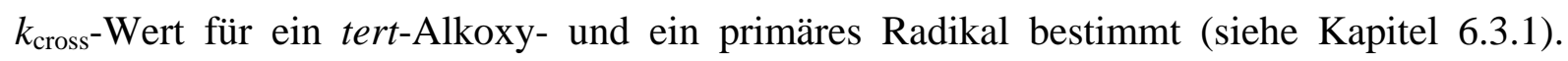
Dieser angenommene Wert ist eher zu groß abgeschätzt, da durch die Delokalisierung der Radikalfunktion im Acyloxyradikal die Reaktivität herabgesetzt sein sollte. Mit diesen Geschwindigkeitskonstanten konnte die in der Ethen-Hochdruckpolymerisation ermittelte Effektivität $\left(f_{\text {Ethen }}(\right.$ TAPA $\left.)=0.69\right)$ nicht erhalten werden. Stattdessen wurde $f_{\text {Käfigprodukte }}=0.79$ erhalten. Die einzige Geschwindigkeitskonstante, die beim Übergang von den tert-Butyl- und 1,1,3,3-Tetramethylbutylperoxyester zu den tert-Amylperoxyester noch nicht in der Modellierung benutzt wurde, ist $k_{\beta, R}$. Deshalb wurde $k_{\beta, R}$ an die experimentell gemessene Initiatoreffektivität angepasst. Mit einem um den Faktor sechs größeren $k_{\beta, \mathrm{R}}=4.0 \cdot 10^{9} \mathrm{~s}^{-1}$ wurde $f_{\text {Ethen }}$ modelliert. Die Aktivierungsenergie aus der DFT-Berechnung müsste um $7 \mathrm{~kJ} \cdot \mathrm{mol}^{-1}$ kleiner sein als die tatsächlich ermittelte um auf diesen Wert zu kommen. Dies könnte ein Effekt der Käfigumgebung sein, der nicht in der DFT-Berechnung berücksichtigt wurde. Mit dieser Geschwindigkeitskonstante wurden alle nachfolgenden Modellierungen durchgeführt. Als einzige Geschwindigkeitskonstante wurde für die restlichen tertAmylperoxyester $k_{\text {cross }}$ angepasst.

In Tabelle 6.5 sind die modellierten Geschwindigkeitskonstanten der Käfigreaktionen für die tert-Amylperoxyester aufgelistet. Für diese sind alle Reaktionswege in Abbildung 6.1 möglich.

\begin{tabular}{ccccc}
\hline Peroxyester & $\boldsymbol{k}_{\text {cross }} / \mathbf{s}^{-\mathbf{1}}$ & $\boldsymbol{k}_{\text {comb,Alkan }} / \mathbf{s}^{-\mathbf{1}}$ & $\boldsymbol{k}_{\text {cross, } \mathbf{R}} / \mathbf{s}^{\mathbf{- 1}}$ & $\boldsymbol{k}_{\text {cross,Säure }} / \mathbf{s}^{\mathbf{- 1}}$ \\
\hline TAPA & - & $5.0 \cdot 10^{10}$ & $5.0 \cdot 10^{9}$ & $\leq 8.0 \cdot 10^{8}$ \\
TAPnB & $8.0 \cdot 10^{8}$ & $2.5 \cdot 10^{10}$ & $3.0 \cdot 10^{9}$ & $\leq 8.0 \cdot 10^{8}$ \\
TAPiB & $4.5 \cdot 10^{9}$ & $1.5 \cdot 10^{10}$ & $5.0 \cdot 10^{9}$ & $\leq 8.0 \cdot 10^{8}$ \\
TAPO & $2.5 \cdot 10^{9}$ & $1.5 \cdot 10^{10}$ & $5.0 \cdot 10^{9}$ & $\leq 8.0 \cdot 10^{8}$ \\
TAPP & $1.2 \cdot 10^{10}$ & $5.0 \cdot 10^{9}$ & $3.0 \cdot 10^{9}$ & $\leq 8.0 \cdot 10^{8}$ \\
\hline
\end{tabular}

Tab.6.5: Die an die im Rahmen dieser Arbeit experimentell bestimmten Initiatoreffektivitäten angepassten Geschwindigkeitskonstanten bei $190{ }^{\circ} \mathrm{C}$ und 2000 bar. 
$k_{\text {comb,Alkan }}$ des Ethylradikals ist stark abhängig vom Kombinationspartner. Die Geschwindigkeitskonstante sinkt um eine Größenordnung zwischen der Kombination mit einem Methylradikal und der Kombination mit einem tert-Butylradikal. Die Kreuzdisproportionierung ist hingegen nahezu konstant und schwankt zwischen 3.0 und $5.0 \cdot 10^{9} \mathrm{~s}^{-1}$.

Die Hauptkäfigprodukte in der Modellierung sind Ether, Alken und Alkan. Ester und Alken (2) werden nur in Spuren gebildet (siehe Tabelle 6.6).

\begin{tabular}{ccccccc}
\hline Peroxyester & $\boldsymbol{x}_{\text {Ether }}$ & $\boldsymbol{x}_{\text {Alken }}$ & $\boldsymbol{x}_{\text {Alkan }}$ & $\boldsymbol{x}_{\text {Säure }}$ & $\boldsymbol{x}_{\text {Ester }}$ & $\boldsymbol{x}_{\text {Alken(2) }}$ \\
\hline TAPA & 0.15 & - & 0.11 & 0.00 & 0.01 & 0.01 \\
TAPnB & 0.15 & 0.03 & 0.09 & 0.00 & 0.01 & 0.01 \\
TAPiB & 0.16 & 0.18 & 0.07 & 0.00 & 0.00 & 0.03 \\
TAPO & 0.17 & 0.11 & 0.08 & 0.00 & 0.00 & 0.03 \\
TAPP & 0.12 & 0.36 & 0.03 & 0.00 & 0.00 & 0.01 \\
\hline
\end{tabular}

Tab.6.6: Stoffmengenanteile der modellierten Käfigprodukte und die mit dem Modell erhaltenden Effektivitäten für 2000 bar.

Die aus dem Stoffmengenanteil der Käfigprodukte zugängliche Effektivität der tert-Amylperoxyester ist in Tabelle 6.7 aufgeführt.

Wie bei den tert-Butylperoxyestern wurden die Effektivitäten von TAPA und TAPnB an den Mittelwert von $f_{\text {Ethen }}$ angepasst.

\begin{tabular}{ccc}
\hline Peroxyester & $\boldsymbol{f}_{\text {Käfigprodukte }}$ & $\boldsymbol{f}_{\text {Ethen }}$ \\
\hline TAPA & 0.72 & 0.69 \\
TAPnB & 0.71 & 0.73 \\
TAPiB & 0.56 & 0.56 \\
TAPO & 0.61 & 0.61 \\
TAPP & 0.48 & 0.48 \\
\hline
\end{tabular}

Tab.6.7: Die an die experimentell bestimmten Initiatoreffektivitäten modellierten Effektivitäten

Zum Vergleich sind in Tabelle 6.8 die modellierten Stoffmengenanteile der Käfigprodukte und die daraus resultierenden Initiatoreffektivitäten aufgelistet, die mit der unveränderten 
Geschwindigkeitskonstante $k_{\beta, R}=6.95 \cdot 10^{8} \mathrm{~s}^{-1}$ und den in Tabelle 6.5 erhaltenden Geschwindigkeitskonstanten modelliert wurden. Alken $_{(2)}$, Säure und Ester wurden nicht erhalten.

\begin{tabular}{cccccc}
\hline Peroxyester & $\boldsymbol{x}_{\text {Ether }}$ & $\boldsymbol{x}_{\text {Alken }}$ & $\boldsymbol{x}_{\text {Alkan }}$ & $\boldsymbol{f}_{\text {Käfigprodukte }}$ & $\boldsymbol{f}_{\text {Ethen }}$ \\
\hline TAPA & 0.19 & - & 0.02 & 0.79 & 0.69 \\
TAPnB & 0.18 & 0.03 & 0.02 & 0.77 & 0.73 \\
TAPiB & 0.18 & 0.20 & 0.02 & 0.60 & 0.56 \\
TAPO & 0.20 & 0.12 & 0.02 & 0.66 & 0.61 \\
TAPP & 0.14 & 0.41 & 0.00 & 0.45 & 0.48 \\
\hline
\end{tabular}

Tab.6.8: Die modellierten Stoffmengenanteile der Käfigprodukte und die daraus

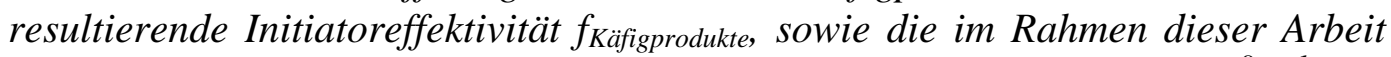
experimentell gemessenen $f_{\text {Ethen. }}$ Zur Modellierung wurde $k_{\beta, R}=6.95 \cdot 10^{8} \mathrm{~s}^{-1}$ und die in Tabelle 6.5 aufgelisteten Geschwindigkeitskonstanten benutzt.

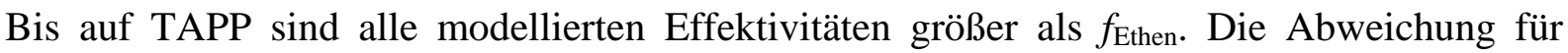
TAPA ist mit 0.1 am größten. Die anderen modellierten Werte befinden sich innerhalb der Messgenauigkeit der experimentell gemessenen Effektivitäten. Dieser Befund würde für ein zu klein bestimmtes $f_{\text {Ethen }}$ (TAPA) sprechen. Dennoch sollte in der DFT-Berechnung von $k_{\beta, R}$ des tert-Amyloxyradikals überprüft werden, ob $E_{\mathrm{A}}$ zu groß bestimmt wurde.

In Tabelle 6.9 sind die Stoffmengenanteile der Käfigprodukte und die Geschwindigkeitskonstanten $k_{\text {cross }}$ aufgelistet, die benötigt werden, um mit $k_{\beta, \mathrm{R}}=6.95 \cdot 10^{8} \mathrm{~s}^{-1}$ die experimentell gemessenen Effektivitäten zu erhalten.

\begin{tabular}{cccccc}
\hline Peroxyester & $\boldsymbol{k}_{\text {cross }} / \mathbf{s}^{-\mathbf{1}}$ & $\boldsymbol{x}_{\text {Ether }}$ & $\boldsymbol{x}_{\text {Alken }}$ & $\boldsymbol{x}_{\text {Alkan }}$ & $\boldsymbol{f}_{\text {Käfigprodukte }}$ \\
\hline TAPnB & $1.5 \cdot 10^{9}$ & 0.18 & 0.07 & 0.02 & 0.73 \\
TAPiB & $5.5 \cdot 10^{9}$ & 0.18 & 0.24 & 0.02 & 0.56 \\
TAPO & $4.0 \cdot 10^{9}$ & 0.19 & 0.19 & 0.01 & 0.61 \\
TAPP & $1.0 \cdot 10^{9}$ & 0.15 & 0.36 & 0.01 & 0.48
\end{tabular}

Tab.6.9: Die modellierten Stoffmengenanteile der Käfigprodukte und die daraus

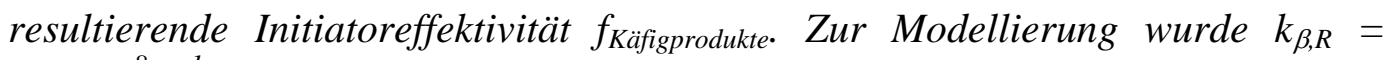
$6.95 \cdot 10^{8} \mathrm{~s}^{-1}$ benutzt. 


\subsubsection{1,1,2,2-Tetramethylpropylperoxyester}

Beim Zerfall von TMPPP entstehen nach der $\beta$-scission des 1,1,2,2-Tetramethylpropyloxyradikals und der Decarboxylierung des Pivaloxyradikals zwei tert-Butylradikale. Für die Terminierung dieser Radikale innerhalb des Lösungsmittelkäfigs wurde von JAUER die Temperaturabhängigkeit der Geschwindigkeitskonstante abgeschätzt [12]. Es wurde gezeigt, dass die in der Gasphase bestimmte leicht negative Temperaturabhängigkeit [1] zu schwach ist, um die Käfigprodukte in den verschiedenen Lösungsmitteln zu modellieren. Aufgrund der Temperaturabhängigkeit der Käfigreaktionen wurde die Modellierung der Effektivität von TMPPP für die Temperatur von $140{ }^{\circ} \mathrm{C}$ durchgeführt. Dies war die mittlere Temperatur, bei der die Initiatoreffektivität von TMPPP in der Ethen-Hochdruckpolymerisation bestimmt wurde. JAUER hat ebenfalls ein Ansatz mit einem Lösungsmittelkäfig genutzt und berechnete die unimolekulare Geschwindigkeitskonstante für die Terminierung von zwei tertButylradikalen bei $140{ }^{\circ} \mathrm{C}$ und 2000 bar $\mathrm{zu} k_{\mathrm{t}}=2 \cdot 8 \cdot 10^{9} \mathrm{~s}^{-1}[12] . k_{\mathrm{t}}$ ist die Summe von $k_{\text {comb,Alkan }}$ und $k_{\text {cross,R. }}$ Mit diesem $k_{\mathrm{t}}$ und dem aus DFT-Berechnungen erhaltenden $k_{\beta, \mathrm{R}}$ wurden zu große Effektivitäten erhalten, weil die nach $\beta$-scission und Decarboxylierung entstandenen tert-Butylradikale zu langsam zu Käfigprodukten kombinieren und disproportionieren. Da mit dieser Terminierungsgeschwindigkeitskonstante die Effektivität in der EthenHochdruckpolymerisation nicht modelliert werden konnte, wurde $k_{\beta, R}$ um den Faktor zehn heruntergesetzt, um die längere Zeit das 1,1,2,2-Tetramethylpropyloxyradikal vorliegen zu haben. Diese Änderung würde eine um $8 \mathrm{~kJ} \cdot \mathrm{mol}^{-1}$ größere Aktivierungsenergie für $k_{\beta, \mathrm{R}}$ bedeuten. Mit $k_{\beta, \mathrm{R}}=2.2 \cdot 10^{10} \mathrm{~s}^{-1}$ wurden die Geschwindigkeitskonstanten, Stoffmengenanteile der Käfigprodukte und Initiatoreffektivitäten erhalten (siehe Tabellen 6.10, 6.11 und 6.12). Durch Modellierung der Effektivität bei der mit TMPPP initiierte EthenHochdruckpolymerisation wurde $k_{\beta, \mathrm{R}}$ bestimmt. Dazu wurde $k_{\text {cross }}$ von TBPP übernommen und $k_{\text {cross,Säure }}$ wurde $k_{\text {cross }}$ gleichgesetzt, weil $k_{\text {cross,Säure }}$ maximal den Wert von $k_{\text {cross }}$ annehmen sollte. $k_{\text {comb,Alkan }}$ und $k_{\text {cross,R }}$ werden von JAUER übernommen. Bei TMPPA wurde $k_{\text {cross,Säure }}$ angepasst.

\begin{tabular}{ccccc}
\hline Peroxyester & $\boldsymbol{k}_{\text {cross }} / \mathbf{s}^{\mathbf{- 1}}$ & $\boldsymbol{k}_{\text {comb,Alkan }} / \mathbf{s}^{-\mathbf{1}}$ & $\boldsymbol{k}_{\text {cross, } \mathbf{R}} / \mathbf{s}^{\mathbf{- 1}}$ & $\boldsymbol{k}_{\text {cross,Säure }} / \mathbf{~ s}^{\mathbf{- 1}}$ \\
\hline TMPPA & - & $8.0 \cdot 10^{9}$ & $5.0 \cdot 10^{9}$ & $1.5 \cdot 10^{10}$ \\
TMPPP & $1.7 \cdot 10^{10}$ & $8.0 \cdot 10^{8}$ & $2.0 \cdot 10^{9}$ & $\leq 1.7 \cdot 10^{10}$ \\
\hline
\end{tabular}

Tab. 6.10: Die an die im Rahmen dieser Arbeit experimentell bestimmten Initiatoreffektivitäten angepassten Geschwindigkeitskonstanten bei 2000 bar und $140{ }^{\circ} \mathrm{C}$. 
Bei TMPPP sind die Hauptkäfigprodukte Alken aus der Kreuzdisproportionierung des 1,1,2,2-Tetramethylpropyloxyradikals mit dem tert-Butylradikal und Alken $_{(2)}$ aus der Selbstdisproportionierung der tert-Butylradikale. Bei TMPPA wird ein großer Anteil Säure bzw. Alken(2) und Alkan gebildet. Zur Berechnung der Initiatoreffektivität wird der Stoffmengenanteil der Säure nicht berücksichtig, weil bereits das bei derselben Reaktion entstehende $\operatorname{Alken}_{(2)}$ in die Berechnung von $f$ eingeht.

\begin{tabular}{ccccccc}
\hline Peroxyester & $\boldsymbol{x}_{\text {Ether }}$ & $\boldsymbol{x}_{\text {Alken }}$ & $\boldsymbol{x}_{\text {Alkan }}$ & $\boldsymbol{x}_{\text {Säure }}$ & $\boldsymbol{x}_{\text {Ester }}$ & $\boldsymbol{x}_{\text {Alken(2) }}$ \\
\hline TMPPA & 0.06 & - & 0.12 & 0.19 & 0.06 & 0.26 \\
TMPPP & 0.08 & 0.32 & 0.03 & 0.00 & 0.00 & 0.07 \\
\hline
\end{tabular}

Tab. 6.11: Stoffmengenanteile der modellierten Käfigprodukte für 2000 bar $140^{\circ} \mathrm{C}$.

\begin{tabular}{ccc}
\hline Peroxyester & $\boldsymbol{f}_{\text {Käfigprodukte }}$ & $\boldsymbol{f}_{\text {Ethen }}$ \\
\hline TMPPA & 0.50 & 0.50 \\
TMPPP & 0.50 & 0.50 \\
\hline
\end{tabular}

Tab.6.12: Die an die experimentell bestimmten Initiatoreffektivitäten modellierten Effektivitäten. Der Stoffmengenanteil an Säure wurde nicht zur Berechnung von $f$ herangezogen.

In Tabelle 6.13, 6.14 und 6.15 sind die modellierten Geschwindigkeitskonstanten, Stoffmengenanteile an Käfigprodukten und die daraus ermittelten Initiatoreffektivitäten aufgelistet, die mit dem ursprünglichen $k_{\beta, R}$ erhalten werden. Für die Modellierung von TMPPA wurde $k_{\text {cross,Säure }}$ angepasst. Bei TMPPP wurde $k_{\text {cross,Säure }}$ von TMPPA und $k_{\text {cross }}$ von

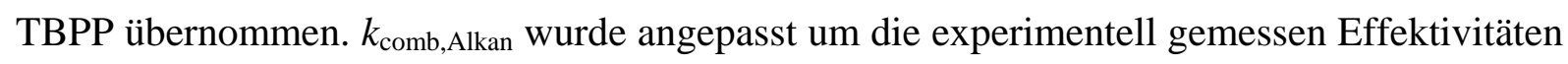
zu erhalten.

\begin{tabular}{ccccc}
\hline Peroxyester & $\boldsymbol{k}_{\text {cross }} / \mathbf{s}^{\mathbf{- 1}}$ & $\boldsymbol{k}_{\text {comb,Alkan }} / \mathbf{s}^{-\mathbf{1}}$ & $\boldsymbol{k}_{\text {cross, } \mathbf{R}} / \mathbf{s}^{\mathbf{- 1}}$ & $\boldsymbol{k}_{\text {cross,Säure }} / \mathbf{~ s}^{\mathbf{- 1}}$ \\
\hline TMPPA & - & $8.0 \cdot 10^{9}$ & $5.0 \cdot 10^{9}$ & $9.0 \cdot 10^{9}$ \\
TMPPP & $1.7 \cdot 10^{10}$ & $2.0 \cdot 10^{9}$ & $6.0 \cdot 10^{9}$ & $9.0 \cdot 10^{9}$ \\
\hline
\end{tabular}

Tab. 6.13: Die an die im Rahmen dieser Arbeit experimentell bestimmten Initiatoreffektivitäten angepassten Geschwindigkeitskonstanten bei 2000 bar und $140{ }^{\circ} \mathrm{C}$. Mit $k_{\beta, R}$ $=2.3 \cdot 10^{11} \mathrm{~s}^{-1}$ modelliert. 


\begin{tabular}{ccccccc}
\hline Peroxyester & $\boldsymbol{x}_{\text {Ether }}$ & $\boldsymbol{x}_{\text {Alken }}$ & $\boldsymbol{x}_{\text {Alkan }}$ & $\boldsymbol{x}_{\text {Säure }}$ & $\boldsymbol{x}_{\text {Ester }}$ & $\boldsymbol{x}_{\text {Alken(2) }}$ \\
\hline TMPPA & 0.00 & - & 0.05 & 0.30 & 0.13 & 0.32 \\
TMPPP & 0.02 & 0.07 & 0.10 & 0.01 & 0.00 & 0.31 \\
\hline
\end{tabular}

Tab. 6.14: Stoffmengenanteile der modellierten Käfigprodukte für 2000 bar $140{ }^{\circ} \mathrm{C}$. Mit $k_{\beta, R}=2.3 \cdot 10^{11} \mathrm{~s}^{-1}$ modelliert.

\begin{tabular}{ccc}
\hline Peroxyester & $\boldsymbol{f}_{\text {Käfigprodukte }}$ & $\boldsymbol{f}_{\text {Ethen }}$ \\
\hline TMPPA & 0.50 & 0.50 \\
TMPPP & 0.50 & 0.50 \\
\hline
\end{tabular}

Tab.6.15: Die an die experimentell bestimmten Initiatoreffektivitäten modellierten Effektivitäten. Der Stoffmengenanteil an Säure wurde nicht zur Berechnung von $f$ herangezogen. Mit $k_{\beta, R}=2.3 \cdot 10^{11} \mathrm{~s}^{-1}$ modelliert.

Im Vergleich zu den in Tabelle 6.9 aufgelisteten Geschwindigkeitskonstanten ist $k_{\text {cross,Säure in }}$

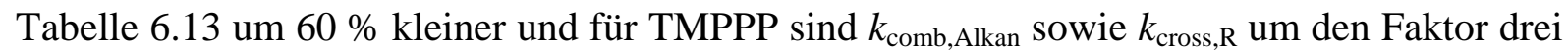
größer als bei JAUER [12] angegeben. Mit beiden Ansätzen können die Effektivitäten aus der Ethen-Hochdruckpolymerisation modelliert werden. Nur die Stoffmengenanteile der Käfigprodukte sind unterschiedlich, so dass mit GC-Untersuchungen der Zersetzungsprodukte des thermischen Zerfalls unterscheiden werden kann, welcher Satz an Geschwindigkeitskonstanten die experimentellen Beobachtung besser beschreibt.

\subsection{Zusammenfassung}

In Kapitel 6.3 wurde gezeigt, dass das kinetische Modell in Abbildung 6.1 die Initiatoreffektivität beschreibt. In diesem Abschnitt werden die Ergebnisse zusammengefasst, mit denen $f$ Ethen genau beschrieben werden kann (angepasste $k_{\beta, R}$ für die tert-Amyl- und 1,1,2,2-Tetramethylpropylperoxyester)

In Tabelle 6.16 sind die gemittelten Geschwindigkeitskonstanten $k_{\text {cross }}$ und $k_{\text {cross,Säure }}$ in Abhängigkeit vom Alkylradikal gezeigt. Wenn ein sauerstoffzentriertes Radikal mit einem kohlenstoffzentrierten Radikal disproportioniert, ist die Geschwindigkeitskonstante nur von

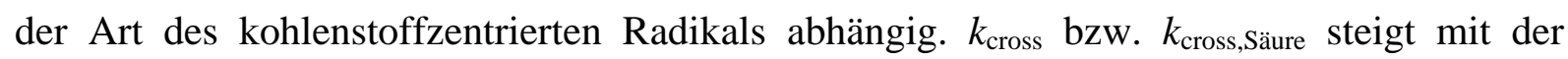
Anzahl der zur radikalischen Funktion $\beta$-ständigen H-Atome. Die Kombinationsgeschwindig- 
keitskonstante $k_{\text {comb }}$ eines sauerstoff- und kohlenstoffzentrierten Radikals ist unabhängig von der Art des Alkylradikals.

Der Anstieg von $k_{\text {cross }}$ und $k_{\mathrm{CO} 2}$ ist verantwortlich für die Abnahme von $f$ der tert-Alkylperoxyester bei Variation der Säureseite (siehe z.B. die tert-Butylperoxyester).

\begin{tabular}{|c|c|c|c|}
\hline & Radikale & & $\boldsymbol{k}_{\text {cross,(Säure) }} / \mathrm{s}^{-1}$ \\
\hline \multirow{4}{*}{$\begin{array}{c}\text { tert-Alkoxy } \\
\text { bzw. } \\
\text { Acyloxy }\end{array}$} & \multirow{4}{*}{+} & Methyl & - \\
\hline & & primär & $8.0 \cdot 10^{8}$ \\
\hline & & sekundär & $3.5 \cdot 10^{9}$ \\
\hline & & tertiär & $1.5 \cdot 10^{10}$ \\
\hline
\end{tabular}

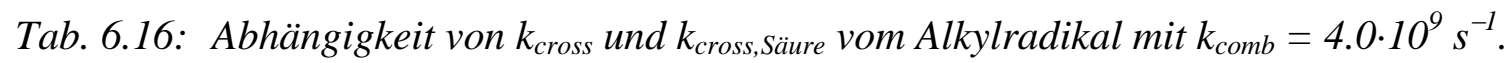

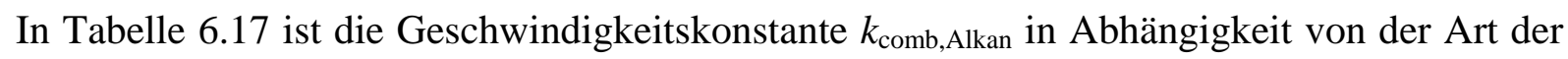
Alkylradikale gezeigt. Die schnellste untersuchte Kombination erfolgt demnach zwischen einem Methylradikal und einem primären Alkylradikal. Die Kombinationsgeschwindigkeitskonstante ist für zwei tertiäre Radikale am kleinsten. $k_{\text {comb,Alkan }}$ dieser zwei Radikalpaare sinkt um fast zwei Größenordnungen von $5.0 \cdot 10^{10}$ auf $8.0 \cdot 10^{8} \mathrm{~s}^{-1}$. Für die primären und tertiären Radikale nimmt $k_{\text {comb,Alkan }}$ um eine Größenordnung vom Methylradikal zum tertiären Radikal $\mathrm{ab}$.

\begin{tabular}{c|cccc}
\hline $\boldsymbol{k}_{\text {comb,Alkan }} / \mathbf{~ s}^{-\mathbf{1}}$ & Methyl & primär & sekundär & tertiär \\
\hline Methyl & - & $5.0 \cdot 10^{10}$ & - & $8.0 \cdot 10^{9}$ \\
primär & $5.0 \cdot 10^{10}$ & $2.0-2.5 \cdot 10^{10}$ & $1.0-1.5 \cdot 10^{10}$ & $4.0-5.0 \cdot 10^{9}$ \\
sekundär & - & $1.0-1.5 \cdot 10^{10}$ & - & - \\
tertiär & $8.0 \cdot 10^{9}$ & $4.0-5.0 \cdot 10^{9}$ & - & $8.0 \cdot 10^{8}\left(140^{\circ} \mathrm{C}\right)$ \\
\hline
\end{tabular}

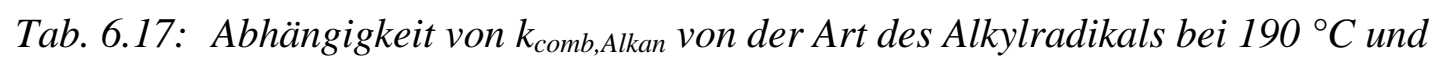
2000 bar.

$k_{\text {cross, } \mathrm{R}}$ ist im Mittel nahezu unabhängig von der Art des Alkylradikals. Der Wert für $190{ }^{\circ} \mathrm{C}$ und 2000 bar liegt bei $4.0 \cdot 10^{9} \mathrm{~s}^{-1}$.

Die schnelle Kombination, durch vermehrt auftretende $\beta$-scission der tert-Alkoxyradikale zu primären Alkylradikalen, verursacht die Abnahme der Initiatoreffektivität der primären tertAlkylperoxyester bei Variation der Alkoholseite (siehe $f_{\mathrm{TBPA}}=0.79$ und $f_{\mathrm{TAPA}}=0.69$ ). 
Bei den tertiären Peroxyestern hingegen bewirkt vermehrt innerhalb des Käfigs auftretende $\beta$-scission eine Steigerung in $f$, da die Käfigreaktionen zweier Alkylradikale langsamer sind als die Käfigreaktionen zwischen einem tert-Alkoxy- und Alkylradikal. Nach der $\beta$-scission des tert-Amyloxy-, 1,1,3,3-Tetramethylbutyloxy- und des 1,1,2,2-Tetramethylpropyloxyradikals im Käfig entstehen weniger Käfigprodukte als bei TBPP, bei dem das tert-Butoxyradikal nicht innerhalb des Käfigs zerfällt (siehe $f_{\text {TBPP }}=0.37$ und $f_{\text {TMPPP }}=0.50$ ).

Bei TMPPA ist die Effektivität klein, weil in diesem Fall ein tert-Butylradikal nach der $\beta$ scission des 1,1,2,2-Tetramethylpropylradikals und ein sauerstoffzentriertes Acetoxyradikal vorliegen, die schnell disproportionieren. Wenn das Acetoxyradikal decarboxyliert, kann das sehr reaktive Methylradikal mit dem tert-Butylradikal schnell kombinieren und disproportionieren $\left(f_{\text {TMPPA }}=0.50\right)$.

Die Modellierungen mit den unveränderten $k_{\beta, R}$-Werten zeigen, dass $f_{\mathrm{Ethen}}(\mathrm{TAPA})$ zu klein bestimmt sein könnte, da die weiteren $f_{\text {Ethen }}$-Werte der tert-Amylperoxyester nicht exakt, aber gut beschrieben werden.

Damit die Modellierung für die 1,1,2,2-Tetramethylpropylperoxyester mit den unveränderten $k_{\beta, \mathrm{R}}$-Werten die experimentell bestimmten Initiatoreffektivitäten ergeben, muss $k_{\mathrm{t}}$ der zwei tert-Butylradikale um den Faktor drei hochgesetzt werden. $k_{\text {cross,Säure }}$ ist in diesem Fall um

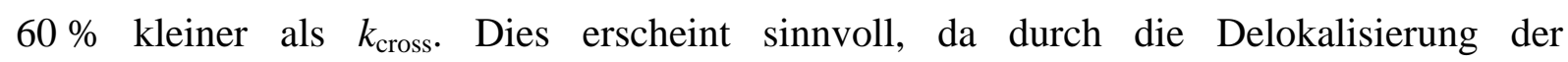
Radikalfunktion im Acyloxyradikal die Reaktivität im Vergleich zum tert-Alkoxyradikal herabgesetzt sein kann. Um genauer Auskunft zu erhalten, welche Geschwindigkeitskonstanten die kinetische Situation korrekt beschreiben, müssten die Stoffmengenanteile der Käfigprodukte analysiert werden.

Der Vergleich experimenteller und berechneter Effektivitäten zeigte, dass die Initiatoreffektivitäten in der Ethen-Hochdruckpolymerisation mit dem kinetischen Modell in Abbildung 6.1 angemessen beschrieben werden können. 


\subsection{Literaturverzeichnis für Kapitel 6}

[1] D. A. Parkes, P. C. Quinn, J. Chem Soc., Faraday Trans. 172 (1976) 1952

[2] J. O. Terry, J. H. Futrell, Canad. J. Chem. 45 (1967) 2327

[3] J. O. Terry, J. H. Futrell, Canad. J. Chem. 46 (1968) 664

[4] J. A. Garcia Dominguez, J. A. Kerr, A. F. Trotman-Dickenson, J. Chem. Soc (1962) 3357

[5] J. Grotewold, J. A. Kerr, J. Chem. Soc (1963) 4337

[6] L. J. Garland, K. D. Bayes, J. Phys. Chem. 94 (1990) 4941

[7] J. A. Kerr, A. F. Trotman-Dickenson, J. Chem. Soc (1960) 1609

[8] P. Becker, M. Buback, J. Sandmann, Macromol. Chem. Phys. 203 (2002) 2113

[9] J. Sandmann, Dissertation, Göttingen (2000)

[10] S. Jauer, persönliche Mitteilung, (2001)

[11] R. A. Sheldon, J. K. Kochi, J. Am. Chem. Soc. 92 (1970) 5175

[12] S. Jauer, Dissertation, Göttingen (2005) 


\section{Kapitel 7}

\section{Ausblick}

Es wurden die Initiatoreffektivitäten $f$ zahlreicher organischer Peroxiden in der EthenHochdruckpolymerisation bestimmt und modelliert.

Es wäre wichtig zu wissen, ob die so erhaltenen kinetischen Daten es erlauben, die Effektivitäten anderer Monomere abzuschätzen. Auch sollten die Einflüsse der Polarität und Viskosität des Reaktionsmediums auf $f$ studiert werden.

Die Druckabhängigkeit der Initiatoreffektivität in der Ethen-Hochdruckpolymerisation deutet an, dass sich der Verlauf von $f$ für TBPP und TBPO bei niedrigeren Drücken asymptotisch dem Wert eins annähert. Mit einem geeigneten Monomer könnte die Druckabhängigkeit von $f$ bis zum Umgebungsdruck gemessen werden. Dies ist mit Ethen nicht möglich, da das Ethen/Polyethylen-Gemisch unterhalb von 1600 bar und $130{ }^{\circ} \mathrm{C}$ inhomogen wird.

Die im Rahmen dieser Arbeit durchgeführten Modellierungen der Initiatoreffektivität von tert-Alkylperoxyester sagen die Stoffmengenanteile der Käfigprodukte beim thermischen Zerfall in $n$-Heptan voraus. Es wäre wünschenswert, wenn die Vorhersagen durch GCUntersuchungen der Käfigprodukte überprüft würden bzw. im Fall der 1,1,2,2-Tetramethylperoxyester die Geschwindigkeitskonstanten noch genauer bestimmt werden könnten. Es wäre lohnend zu überprüfen, ob das in Kapitel 6 vorgestellte kinetische Modell die Effektivität von bisher nicht in der Ethen-Hochdruckpolymerisation untersuchten organischen Peroxiden (z.B. von Peroxycarbonaten und Peroxydicarbonate) richtig vorhersagt. 


\section{Anhang A}

\section{Verzeichnis der Abkürzungen}

A

BTMHP

$c_{0}$

$c_{\mathrm{i}}$

$C_{\text {INI }}$

$C_{\mathrm{INI}, 0}$

$C_{\text {INI, zersetzt }}$

CSTR

CTA

D

DFT

DTAP

DTBP

E

$E_{\mathrm{A}}(0$ bar $)$

$E_{\mathrm{A}}$

$f$

$f_{\text {Ethen }}$

$f_{\mathrm{i}}$

$f_{\text {Käfigprodukte }}$

GC

$\eta$

$\eta_{0}$

ID

INI

IP

$k$

$k_{\beta}$
Stoßfaktor, präexponentieller Faktor

Bis-3,5,5-trimethylhexanoylperoxid

Konzentration zur Zeit $t=0$

Konzentration der Komponente i

aktuelle Konzentration des Initiators im CSTR

Konzentration des Initiators im Einlaßstrom des CSTR

„Konzentration“ der unter Reaktionsbedingungen zerfallenden

Initiatormolekülen

kontinuierlich betriebener Rührkessel mit idealer Rückvermischung

Kettenübertragungsreagenz

Diffusionskonstante

Dichte Funktional Theorie (Theorie für quantenmechanische Berechnungen der

Geometrie und Energetik eines Teilchens)

Di-tert-amylperoxid

Di-tert-butylperoxid

Ethen

Aktivierungsenergie für verschwindenden Druck

Aktivierungsenergie

Initiatoreffektivität

Initiatoreffektivität gemessen in der Ethen-Hochdruckpolymerisation

Stoffmengenanteil der Komponente i im Einlaßstrom des CSTR

Initiatoreffektivität berechnet aus den Käfigprodukten

Gaschromatographie

Viskosität

Viskosität bei verschwindenen Druck

spezifischer Initiatorverbrauch (eng.: initiator demand)

Initiatormolekül

Initiatorproduktivität

allgemein: Geschwindigkeitskonstante einer Elementarreaktion

Geschwindigkeitskonstante für die $\beta$-scission eines tert-Alkoxyradikals 


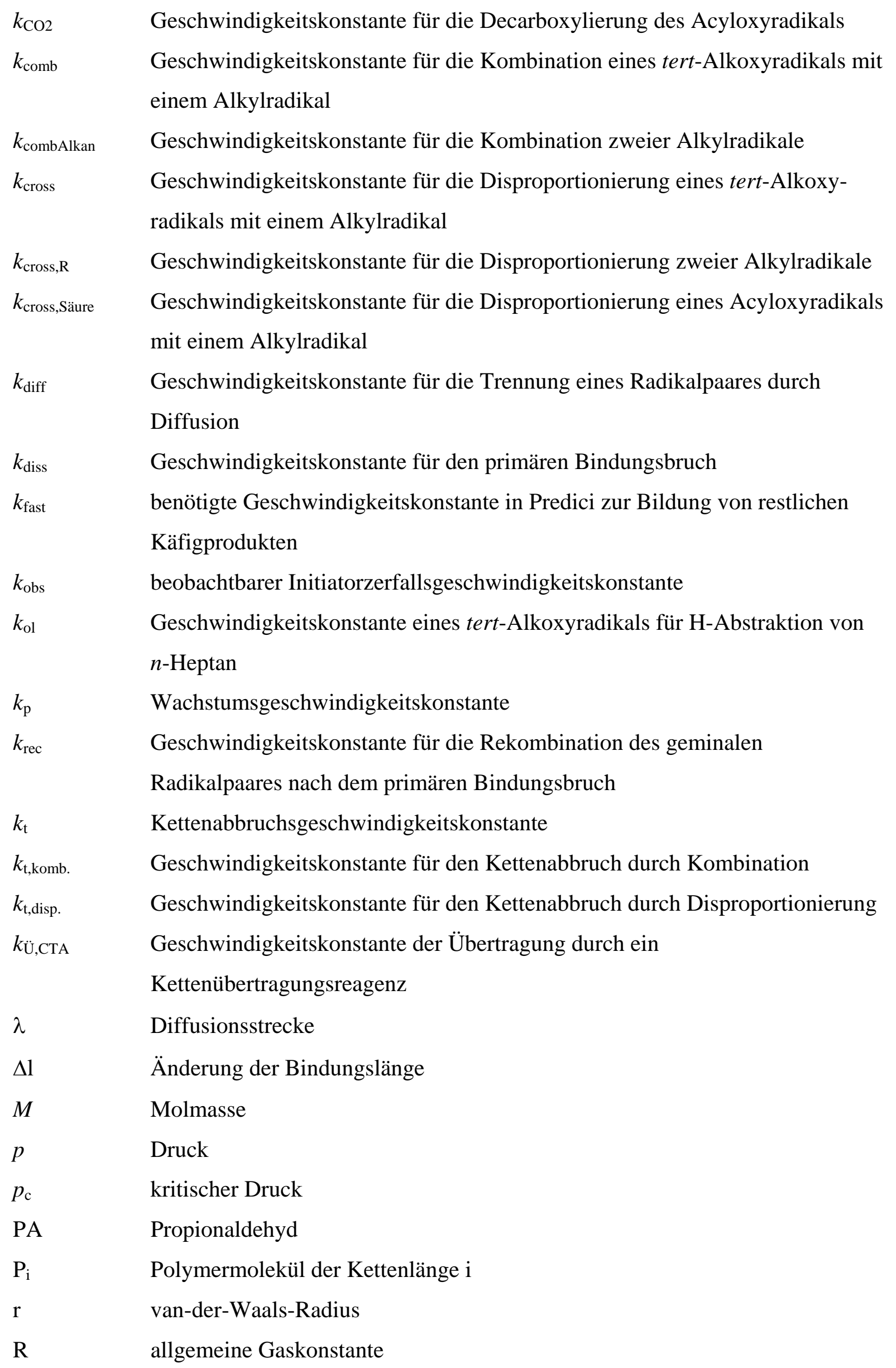




$\begin{array}{ll}\mathrm{R}_{0} & \text { Radikal der Kettenlänge } 0 \\ \rho_{\mathrm{A}} & \text { Dichte des Reaktionsmediums am Reaktorauslass des CSTR } \\ \rho_{\mathrm{C}} & \text { kritische Dichte } \\ \rho_{\mathrm{E}} & \text { Dichte des Reaktionsmediums am Reaktoreinlass des CSTR } \\ \mathrm{R}_{\mathrm{i}} & \text { Radikal der Kettenlänge i } \\ \rho_{\mathrm{r}} & \text { reduzierte Dichte } \\ t & \text { Zeit } \\ \tau & \text { Verweilzeit im CSTR } \\ \tau & \text { Zeit zur diffusiven Trennung zweier Teilchen } \\ T & \text { Temperatur } \\ \text { TAPA } & \text { tert-Amylperoxyacetat }\end{array}$

TAPiB tert-Amylperoxy-iso-butanoat

TAPnB tert-Amylperoxy- $n$-butanoat

TAPO tert-Amylperoxy-2-ethylhexanoat

TAPP tert-Amylperoxypivalat

TATBP tert-Amyl-tert-butylperoxid

TBPA tert-Butylperoxyacetat

TBPiB tert-Butylperoxy-iso-butanoat

TBPnB tert-Butylperoxy-n-butanoat

TBPO tert-Butylperoxy-2-ethylhexanoat

TBPP tert-Butylperoxypivalat

TBPPent tert-Butylperoxypentanoat

$T_{\mathrm{C}} \quad$ kritische Temperatur

$T_{\text {Mantel } \quad \text { Manteltemperatur des CSTR }}$

TMBPA 1,1,3,3-Tetramethylpropylperoxyacetat

TMBPO 1,1,3,3-Tetramethylpropyl-2-ethylhexanoat

TMBPP 1,1,3,3-Tetramethylbutylperoxypivalat

TMPPA 1,1,2,2-Tetramethylpropylperoxyacetat

TMPPP 1,1,2,2-Tetramethylpropylperoxypivalat

TMPTBP 1,1,2,2-Tetramethylpropyl-tert-butylperoxid

$T_{\text {opt }} \quad$ Temperatur, bei der der maximal erreichbare Umsatz erreicht wird

$T_{\mathrm{r}} \quad$ reduzierte Temperatur

$U \quad$ Bruttoumsatz im CSTR in \% 
$\Delta U \quad$ korrigierter Umsatz in \%; Differenz von Bruttoumsatz und thermischen

Grundumsatz

$\Delta U_{\mathrm{r}} \quad$ reduzierte Umsatz

$V \quad$ Reaktorvolumen

V molares Volumen

$\Delta V^{\ddagger} \quad$ Aktivierungsvolumen

$x_{\mathrm{i}} \quad$ Stoffmengenanteil der Komponente i 


\section{Anhang B}

\section{Untersuchung zur Initiatoreffektivität}

1) Initiatorcocktail TBPO/BTMHP

\begin{tabular}{cccc}
\hline$T /{ }^{\circ} \mathrm{C}$ & $U / \%$ & $\Delta U / \%$ & $f_{\text {BTMHP }}$ \\
\hline 140 & 4.19 & 3.78 & 0.46 \\
141 & 4.10 & 3.69 & 0.37 \\
141 & 4.22 & 3.81 & 0.43 \\
151 & 4.92 & 4.50 & 0.36 \\
152 & 5.05 & 4.63 & 0.38 \\
153 & 4.94 & 4.52 & 0.30 \\
177 & 7.61 & 6.80 & 0.42 \\
177 & 7.58 & 6.77 & 0.41 \\
183 & 7.97 & 6.69 & 0.25 \\
185 & 7.85 & 6.58 & 0.18 \\
184 & 7.97 & 6.69 & 0.23 \\
188 & 7.60 & 5.90 & -0.01 \\
189 & 7.72 & 6.02 & 0.00 \\
189 & 7.71 & 6.01 & 0.00 \\
200 & 7.70 & 4.95 & -0.29 \\
200 & 7.73 & 4.98 & -0.28 \\
\hline
\end{tabular}

Tab. B.1: Ergebnisse der Ethen-Homopolymerisation mit dem Initiatorcocktail TBPO/BTMHP im CSTR; $c(\text { TBPO })_{0}=8.5 \cdot 10^{-6} \mathrm{~mol} \mathrm{~L}^{-1} ; c(\text { BTMHP })_{0}=8.6 \cdot 10^{-6}$ $\mathrm{mol} \mathrm{L} \mathrm{L}^{-1} ; f_{P A}=3.2 \cdot 10^{-3} ;$ Massenfluss Ethen $=706 \mathrm{~g} \mathrm{~h}^{-1}$.

\section{2) Initiatorcocktail TBPA/TBPP}

\begin{tabular}{cccc}
\hline$T /{ }^{\circ} \mathrm{C}$ & $U / \%$ & $\Delta U / \%$ & $f_{\mathrm{TBPP}}$ \\
\hline 160 & 5.53 & 4.87 & 0.52 \\
160 & 5.42 & 4.76 & 0.49 \\
175 & 6.64 & 5.63 & 0.33
\end{tabular}




\begin{tabular}{llll}
176 & 6.63 & 5.62 & 0.31 \\
195 & 7.74 & 5.77 & 0.04 \\
195 & 7.67 & 5.71 & 0.03 \\
209 & 8.35 & 6.42 & -0.01 \\
209 & 8.33 & 6.39 & -0.02 \\
218 & 7.46 & 5.33 & -0.18 \\
218 & 7.42 & 5.29 & -0.18 \\
217 & 7.39 & 5.26 & -0.18 \\
\hline
\end{tabular}

Tab. B.2: $\quad$ Ergebnisse der Ethen-Homopolymerisation mit dem Initiatorcocktail TBPP/TBPA im CSTR; $c(\text { TBPP })_{0}=9.8 \cdot 10^{-6} \mathrm{~mol} \mathrm{~L} \mathrm{~L}^{-1} \mathrm{c}(\mathrm{TBPA})_{0}=5.0 \cdot 10^{-6} \mathrm{~mol} \mathrm{~L} \mathrm{C}^{-1} ; f_{\mathrm{PA}}=$ $3.2 \cdot 10^{-3}$; Massenfluss Ethen $=706 \mathrm{~g} \mathrm{~h}^{-1}$.

\section{3) tert-Amyl-tert-butylperoxid}

\begin{tabular}{cccc}
\hline$T /{ }^{\circ} \mathrm{C}$ & $U / \%$ & $\Delta U / \%$ & $f$ \\
\hline 197 & 6.29 & 5.53 & 1.21 \\
197 & 6.20 & 5.43 & 1.17 \\
211 & 8.00 & 6.73 & 1.12 \\
212 & 7.96 & 6.69 & 1.08 \\
235 & 10.02 & 8.12 & 0.92 \\
235 & 10.09 & 8.19 & 0.93 \\
248 & 10.79 & 8.47 & 0.78 \\
249 & 11.21 & 8.89 & 0.84 \\
249 & 11.00 & 8.68 & 0.80 \\
258 & 11.32 & 8.61 & 0.69 \\
257 & 11.38 & 8.67 & 0.71 \\
\hline
\end{tabular}

Tab. B.3: Ergebnisse der Ethen-Homopolymerisation mit TATBP im CSTR; $C_{I N I, 0}=$ $3.9 \cdot 10^{-6} \mathrm{~mol} \mathrm{~L}^{-1} ; f_{P A}=3.2 \cdot 10^{-3}$; Massenfluss Ethen $=706 \mathrm{~g} \mathrm{~h}^{-1}$.

\section{4) 1,1,2,2-Tetramethylpropyl-tert-Butylperoxid}

\begin{tabular}{cccc}
\hline$T /{ }^{\circ} \mathrm{C}$ & $U / \%$ & $\Delta U / \%$ & $f$ \\
\hline 179 & 3.58 & 3.14 & 0.89 \\
179 & 3.56 & 3.12 & 0.87
\end{tabular}




\begin{tabular}{llll}
190 & 4.29 & 3.56 & 0.78 \\
190 & 4.18 & 3.44 & 0.71 \\
200 & 5.47 & 3.87 & 0.67 \\
200 & 5.53 & 3.93 & 0.69 \\
212 & 6.48 & 4.58 & 0.70 \\
212 & 6.40 & 4.50 & 0.68 \\
224 & 7.19 & 4.91 & 0.63 \\
224 & 7.09 & 4.82 & 0.60 \\
237 & 7.78 & 4.69 & 0.44 \\
237 & 7.69 & 4.61 & 0.43 \\
\hline
\end{tabular}

Tab. B.4: Ergebnisse der Ethen-Homopolymerisation mit TMPTBP im CSTR; $c_{I N I, 0}=$ 2.6 $10^{-6} \mathrm{~mol} \mathrm{~L}^{-1} ; f_{P A}=3.2 \cdot 10^{-3}$; Massenfluss Ethen $=706 \mathrm{~g} \mathrm{~h}^{-1}$.

5) tert-Amylperoxyacetat

\begin{tabular}{cccc}
\hline$T /{ }^{\circ} \mathrm{C}$ & $U / \%$ & $\Delta U / \%$ & $f$ \\
\hline 167 & 4.81 & 4.20 & 0.74 \\
167 & 4.71 & 4.10 & 0.71 \\
188 & 7.00 & 6.20 & 0.70 \\
189 & 6.93 & 6.13 & 0.67 \\
196 & 8.27 & 6.56 & 0.63 \\
197 & 8.20 & 6.49 & 0.60 \\
208 & 10.60 & 8.28 & 0.75 \\
210 & 10.28 & 7.96 & 0.67 \\
213 & 10.91 & 8.59 & 0.72 \\
220 & 9.36 & 6.38 & 0.35 \\
\hline
\end{tabular}

Tab. B.5: Ergebnisse der Ethen-Homopolymerisation mit TAPA im CSTR; $C_{I N I, 0}=$ $4.4 \cdot 10^{-6} \mathrm{~mol} \mathrm{~L}^{-1} ; f_{P A}=3.2 \cdot 10^{-3}$; Massenfluss Ethen $=706 \mathrm{~g} \mathrm{~h}^{-1}$.

6) tert-Amylperoxy-n-butyrat

\begin{tabular}{cccc}
\hline$T /{ }^{\circ} \mathrm{C}$ & $U / \%$ & $\Delta U / \%$ & $f$ \\
\hline 166 & 4.72 & 4.33 & 0.78 \\
166 & 4.75 & 4.36 & 0.79
\end{tabular}




$\begin{array}{llll}180 & 6.62 & 5.86 & 0.80 \\ 181 & 6.63 & 5.87 & 0.77 \\ 193 & 7.09 & 6.55 & 0.68 \\ 193 & 7.13 & 6.59 & 0.69 \\ 206 & 8.96 & 7.59 & 0.66 \\ 207 & 9.11 & 7.74 & 0.68 \\ 217 & 9.99 & 7.66 & 0.52 \\ 217 & 9.64 & 7.32 & 0.48 \\ 218 & 9.26 & 6.94 & 0.43\end{array}$

Tab. B.6: Ergebnisse der Ethen-Homopolymerisation mit TAPnB im CSTR; $c_{I N I, 0}=$ 8.6 $10^{-6} \mathrm{~mol} \mathrm{~L}{ }^{-1} ; f_{P A}=3.2 \cdot 10^{-3}$; Massenfluss Ethen $=706 \mathrm{~g} \mathrm{~h}^{-1}$.

7) tert-Amylperoxy-iso-butyrat

\begin{tabular}{cccc}
\hline$T /{ }^{\circ} \mathrm{C}$ & $U / \%$ & $\Delta U / \%$ & $f$ \\
\hline 138 & 2.30 & 2.14 & 0.61 \\
138 & 2.29 & 2.13 & 0.60 \\
148 & 3.02 & 2.75 & 0.55 \\
149 & 3.03 & 2.75 & 0.52 \\
160 & 4.36 & 3.93 & 0.68 \\
160 & 4.31 & 3.88 & 0.66 \\
172 & 4.78 & 4.12 & 0.512 \\
172 & 4.70 & 4.05 & 0.50 \\
183 & 5.88 & 4.71 & 0.52 \\
183 & 5.87 & 4.70 & 0.52 \\
194 & 6.18 & 4.33 & 0.34 \\
195 & 5.96 & 4.11 & 0.30 \\
\hline
\end{tabular}

Tab. B.7: Ergebnisse der Ethen-Homopolymerisation mit TAPiB im CSTR; $c_{I N I, 0}=$ $7.410^{-6} \mathrm{~mol} \mathrm{~L}^{-1} ; f_{P A}=3.2 \cdot 10^{-3} ;$ Massenfluss Ethen $=706 \mathrm{~g} \mathrm{~h}^{-1}$.

8) tert-Amylperoxy-2-ethylhexanoat

\begin{tabular}{cccc}
\hline$T /{ }^{\circ} \mathrm{C}$ & $U / \%$ & $\Delta U / \%$ & $f$ \\
\hline 144 & 2.97 & 2.83 & 0.63
\end{tabular}




\begin{tabular}{llll}
144 & 3.12 & 2.98 & 0.70 \\
155 & 3.73 & 3.51 & 0.64 \\
154 & 3.46 & 3.25 & 0.56 \\
155 & 3.96 & 3.75 & 0.72 \\
165 & 4.42 & 3.99 & 0.60 \\
166 & 4.36 & 3.93 & 0.57 \\
176 & 5.01 & 4.31 & 0.52 \\
176 & 4.96 & 4.26 & 0.51 \\
187 & 4.61 & 3.97 & 0.34 \\
187 & 4.54 & 3.90 & 0.33 \\
\hline
\end{tabular}

Tab. B.8: Ergebnisse der Ethen-Homopolymerisation mit TAPO im CSTR; $c_{I N I, 0}=$ $7.110^{-6} \mathrm{~mol} \mathrm{~L}^{-1} ; f_{P A}=3.2 \cdot 10^{-3}$; Massenfluss Ethen $=706 \mathrm{~g} \mathrm{~h}^{-1}$.

\section{9) tert-Amylperoxypivalat}

\begin{tabular}{cccc}
\hline$T /{ }^{\circ} \mathrm{C}$ & $U / \%$ & $\Delta U / \%$ & $f$ \\
\hline 136 & 4.66 & 4.41 & 0.51 \\
137 & 4.82 & 4.57 & 0.53 \\
136 & 4.86 & 4.61 & 0.56 \\
147 & 5.22 & 4.95 & 0.45 \\
147 & 5.14 & 4.87 & 0.44 \\
158 & 6.11 & 5.69 & 0.44 \\
159 & 6.21 & 5.79 & 0.45 \\
170 & 6.08 & 5.45 & 0.29 \\
171 & 6.28 & 5.65 & 0.31 \\
\hline
\end{tabular}

Tab. B.9: Ergebnisse der Ethen-Homopolymerisation mit TAPP im CSTR; $c_{I N I, 0}=$ $2.3 \cdot 10^{-5} \mathrm{~mol} \mathrm{~L}^{-1} ; f_{P A}=3.2 \cdot 10^{-3} ;$ Massenfluss Ethen $=706 \mathrm{~g} \mathrm{~h}^{-1}$.

10) 1,1,3,3-Tetramethylbutylperoxyacetat

\begin{tabular}{cccc}
\hline$T /{ }^{\circ} \mathrm{C}$ & $U / \%$ & $\Delta U / \%$ & $f$ \\
\hline 166 & 5.26 & 4.79 & 0.80 \\
166 & 5.15 & 4.68 & 0.77
\end{tabular}




\begin{tabular}{llll}
180 & 7.20 & 6.13 & 0.79 \\
181 & 7.22 & 6.14 & 0.77 \\
195 & 8.49 & 7.22 & 0.75 \\
198 & 8.56 & 7.29 & 0.70 \\
208 & 9.04 & 7.31 & 0.56 \\
208 & 9.03 & 7.30 & 0.56 \\
\hline
\end{tabular}

Tab. B.10: Ergebnisse der Ethen-Homopolymerisation mit TMBPA im CSTR; $c_{I N I, 0}=$ $8.9 \cdot 10^{-6} \mathrm{~mol} \mathrm{~L}{ }^{-1} ; f_{P A}=3.2 \cdot 10^{-3}$; Massenfluss Ethen $=706 \mathrm{~g} \mathrm{~h}^{-1}$.

11) 1,1,3,3-Tetramethylbutylperoxy-2-ethylhexanoat

\begin{tabular}{cccc}
\hline$T /{ }^{\circ} \mathrm{C}$ & $U / \%$ & $\Delta U / \%$ & $f$ \\
\hline 146 & 3.33 & 2.87 & 0.54 \\
146 & 3.43 & 2.96 & 0.57 \\
155 & 3.77 & 3.36 & 0.56 \\
167 & 4.61 & 4.00 & 0.57 \\
167 & 4.58 & 3.97 & 0.56 \\
180 & 4.98 & 4.13 & 0.44 \\
180 & 4.98 & 4.13 & 0.44 \\
180 & 5.04 & 4.19 & 0.45 \\
188 & 5.10 & 3.82 & 0.32 \\
188 & 4.88 & 3.61 & 0.28 \\
\hline
\end{tabular}

Tab. B.11: Ergebnisse der Ethen-Homopolymerisation mit TMBPO im CSTR; $C_{I N I, 0}=$ $7.0 \cdot 10^{-6} \mathrm{~mol} \mathrm{~L}^{-1} ; f_{P A}=3.2 \cdot 10^{-3}$; Massenfluss Ethen $=706 \mathrm{~g} \mathrm{~h}^{-1}$.

\begin{tabular}{cccc}
\hline$T /{ }^{\circ} \mathrm{C}$ & $U / \%$ & $\Delta U / \%$ & $f$ \\
\hline 141 & 4.27 & 3.91 & 0.63 \\
142 & 4.29 & 3.94 & 0.62 \\
151 & 5.33 & 4.90 & 0.67 \\
151 & 5.55 & 5.12 & 0.73 \\
151 & 5.04 & 4.61 & 0.60 \\
163 & 6.22 & 5.67 & 0.70 \\
163 & 6.36 & 5.81 & 0.73 \\
176 & 7.15 & 6.51 & 0.60
\end{tabular}




\begin{tabular}{llll}
177 & 7.22 & 6.58 & 0.60 \\
186 & 7.39 & 6.47 & 0.47 \\
186 & 7.48 & 6.56 & 0.48 \\
\hline
\end{tabular}

Tab. B.12: Ergebnisse der Ethen-Homopolymerisation mit TMBPO im CSTR; $C_{I N I, 0}=$ $1.4 \cdot 10^{-5} \mathrm{~mol} \mathrm{~L}{ }^{-1} ; f_{P A}=3.2 \cdot 10^{-3} ;$ Massenfluss Ethen $=706 \mathrm{~g} \mathrm{~h}^{-1}$.

12) 1,1,3,3-Tetramethylbutylperoxypivalat

\begin{tabular}{cccc}
\hline$T /{ }^{\circ} \mathrm{C}$ & $U / \%$ & $\Delta U / \%$ & $f$ \\
\hline 130 & 4.33 & 4.24 & 0.55 \\
130 & 3.99 & 3.90 & 0.47 \\
143 & 5.21 & 4.97 & 0.50 \\
143 & 5.07 & 4.83 & 0.47 \\
153 & 5.41 & 4.97 & 0.39 \\
153 & 5.46 & 5.03 & 0.40 \\
\hline
\end{tabular}

Tab. B.13: Ergebnisse der Ethen-Homopolymerisation mit TMBPP im CSTR; $c_{I N I, 0}=$ 2.3 $10^{-5} \mathrm{~mol} \mathrm{~L}{ }^{-1} ; f_{P A}=3.2 \cdot 10^{-3} ;$ Massenfluss Ethen $=706 \mathrm{~g} \mathrm{~h}^{-1}$.

\section{3) 1,1,2,2-Tetramethylpropylperoxypivalat}

\begin{tabular}{cccc}
\hline$T /{ }^{\circ} \mathrm{C}$ & $U / \%$ & $\Delta U / \%$ & $f$ \\
\hline 129 & 2.78 & 2.38 & 0.50 \\
129 & 2.73 & 2.33 & 0.48 \\
137 & 3.24 & 2.81 & 0.52 \\
137 & 3.22 & 2.79 & 0.52 \\
147 & 3.41 & 2.93 & 0.42 \\
147 & 3.32 & 2.83 & 0.39 \\
147 & 3.42 & 2.93 & 0.42 \\
147 & 3.37 & 2.89 & 0.41 \\
156 & 3.14 & 2.42 & 0.22 \\
156 & 3.34 & 2.62 & 0.26 \\
\hline
\end{tabular}

Tab. B.14: Ergebnisse der Ethen-Homopolymerisation mit TMPPP im CSTR; $c_{I N I, 0}=$

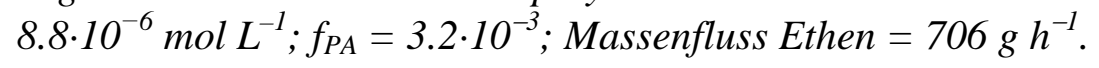




\section{4) tert-Butylperoxypivalat, Druckabhängigkeit}

\begin{tabular}{ccccc}
\hline$T /{ }^{\circ} \mathrm{C}$ & $p /$ bar & $U / \%$ & $\Delta U / \%$ & $f$ \\
\hline 166 & 1600 & 5.35 & 5.09 & 0.53 \\
167 & 1600 & 5.38 & 5.11 & 0.52 \\
168 & 1800 & 6.73 & 6.36 & 0.51 \\
168 & 1800 & 6.63 & 6.27 & 0.49 \\
163 & 2000 & 7.24 & 6.72 & 0.41 \\
163 & 2000 & 7.18 & 6.66 & 0.40 \\
168 & 2250 & 9.05 & 8.47 & 0.34 \\
169 & 2250 & 8.93 & 8.35 & 0.32 \\
172 & 2500 & 10.15 & 9.37 & 0.22 \\
175 & 2500 & 10.21 & 9.43 & 0.21 \\
\hline
\end{tabular}

Tab. B.15: Ergebnisse der Ethen-Homopolymerisation mit TBPP im CSTR; $c_{I N I, 0}=$ 2.3 $10^{-5} \mathrm{~mol} \mathrm{~L}{ }^{-1} ; f_{P A}=3.2 \cdot 10^{-3} ;$ Massenfluss Ethen $=706 \mathrm{~g} \mathrm{~h}^{-1}$.

\begin{tabular}{ccccc}
\hline$T /{ }^{\circ} \mathrm{C}$ & $p /$ bar & $U / \%$ & $\Delta U / \%$ & $f$ \\
\hline 157 & 1600 & 3.56 & 3.44 & 0.67 \\
157 & 1600 & 3.42 & 3.30 & 0.62 \\
156 & 1800 & 4.23 & 3.89 & 0.53 \\
157 & 1800 & 4.38 & 4.05 & 0.56 \\
157 & 1800 & 4.30 & 3.97 & 0.54 \\
156 & 2000 & 4.80 & 4.30 & 0.39 \\
156 & 2000 & 4.82 & 4.33 & 0.39 \\
158 & 2250 & 5.96 & 5.45 & 0.35 \\
158 & 2250 & 5.84 & 5.33 & 0.33 \\
159 & 2250 & 5.93 & 5.41 & 0.33 \\
160 & 2500 & 7.00 & 6.34 & 0.26 \\
161 & 2500 & 7.05 & 6.39 & 0.26 \\
\hline
\end{tabular}

Tab. B.16: Ergebnisse der Ethen-Homopolymerisation mit TBPP im CSTR; $c_{I N I, 0}=$ $1.2 \cdot 10^{-5} \mathrm{~mol} \mathrm{~L}^{-1} ; f_{P A}=3.2 \cdot 10^{-3} ;$ Massenfluss Ethen $=706 \mathrm{~g} \mathrm{~h}^{-1}$. 


\section{5) tert-Butylperoxy-2-ethylhexanoat, Druckabhängigkeit}

\begin{tabular}{ccccc}
\hline$T /{ }^{\circ} \mathrm{C}$ & $p /$ bar & $U / \%$ & $\Delta U / \%$ & $f$ \\
\hline 171 & 1600 & 3.22 & 2.72 & 0.66 \\
171 & 1600 & 3.25 & 2.75 & 0.67 \\
173 & 1800 & 3.92 & 3.39 & 0.60 \\
173 & 1800 & 4.12 & 3.59 & 0.66 \\
173 & 1800 & 4.01 & 3.49 & 0.63 \\
170 & 2000 & 4.68 & 3.89 & 0.50 \\
171 & 2000 & 4.79 & 4.00 & 0.52 \\
173 & 2250 & 6.13 & 5.44 & 0.54 \\
174 & 2250 & 6.18 & 5.49 & 0.54 \\
176 & 2500 & 6.81 & 5.74 & 0.34 \\
177 & 2500 & 6.87 & 5.79 & 0.34 \\
\hline
\end{tabular}

Tab. B.17: Ergebnisse der Ethen-Homopolymerisation mit TBPO im CSTR; $c_{I N I, 0}=$ $5.6 \cdot 10^{-6} \mathrm{~mol} \mathrm{~L}^{-1} ; f_{P A}=3.2 \cdot 10^{-3} ;$ Massenfluss Ethen $=706 \mathrm{~g} \mathrm{~h}^{-1}$.

16) Di-tert-butylperoxid, Druckabhängigkeit

\begin{tabular}{ccccc}
\hline$T /{ }^{\circ} \mathrm{C}$ & $p /$ bar & $U / \%$ & $\Delta U / \%$ & $f$ \\
\hline 199 & 1600 & 2.68 & 2.56 & 0.95 \\
199 & 1600 & 2.73 & 2.62 & 0.99 \\
200 & 1800 & 4.36 & 3.69 & 1.12 \\
200 & 1800 & 4.33 & 3.66 & 1.10 \\
200 & 2000 & 4.17 & 4.03 & 0.91 \\
201 & 2000 & 4.28 & 4.15 & 0.92 \\
201 & 2250 & 5.91 & 5.56 & 0.95 \\
201 & 2250 & 5.73 & 5.38 & 0.89 \\
203 & 2450 & 7.77 & 6.38 & 0.92 \\
203 & 2450 & 7.77 & 6.39 & 0.92 \\
\hline
\end{tabular}

Tab. B.18: Ergebnisse der Ethen-Homopolymerisation mit DTBP im CSTR; $c_{I N I, 0}=$ $2.8 \cdot 10^{-6} \mathrm{~mol} \mathrm{~L}^{-1} ; f_{P A}=3.2 \cdot 10^{-3}$; Massenfluss Ethen $=706 \mathrm{~g} \mathrm{~h}^{-1}$. 


\section{Anhang C}

\section{Berechnung der kinetischen Koeffizienten und der Dichte für das Monomere Ethen}

Berechnung der Dichte im Reaktor anhand von Ethen/Polyethylen-Dichtedaten [1]

Die Dichte im Reaktor berechnet sich nach folgender Formel:

$$
\rho=\frac{1}{\left(1-g_{\mathrm{P}}\right) \cdot \rho_{\mathrm{E}}^{-1}+g_{\mathrm{P}} \cdot \rho_{\mathrm{P}}^{-1}}
$$

mit: $\rho_{\mathrm{E}}=1995.85-601.2 \cdot \lg \left(\frac{p}{1000}\right)+593.3 \cdot \lg \left(\frac{1}{T}\right)-335.8 \cdot \lg \left(\frac{p}{1000}\right) \cdot \lg \left(\frac{1}{T}\right)$

$$
\rho_{\mathrm{P}}=\left(9.61 \cdot 10^{-4}+7.0 \cdot 10^{-7} \cdot \mathrm{T}-5.3 \cdot 10^{-8} \cdot \mathrm{p}\right)^{-1}(\mathrm{D} .3)
$$

Dabei ist : $\rho$ die Dichte im Reaktor in $\mathrm{g} \mathrm{L}^{-1}$

$g_{\mathrm{P}}$ der Gewichtsanteil Polymer im Reaktor

$\rho_{\mathrm{E}}$ die Dichte von Ethen unter Reaktionsbedingungen in $\mathrm{g} \mathrm{L}^{-1}$

$\rho_{\mathrm{P}}$ die Dichte des Polymeren unter Reaktionsbedingungen in $\mathrm{g} \mathrm{L}^{-1}$

$p$ der Druck in bar

$T$ die absolute Temperatur in $\mathrm{K}$

Berechnung der kinetischen Koeffizienten $k_{\mathrm{p}}$ und $\boldsymbol{k}_{\mathrm{t}}$ für Ethen als Funktion von $p$, $T$ und $g_{\mathrm{P}}$

Die angegebenen Formeln zur Berechnung der Koeffizienten wurden von SCHWEER abgeleitet [2]. Zunächst werden für den Wachstums- und Kettenabbruchskoeffizienten Werte für verschwindenden Umsatz in Abhängigkeit von Druck und Temperatur, $k_{\mathrm{p}}^{0}$ und $k_{\mathrm{t}}^{0}$, berechnet:

$$
\begin{aligned}
& k_{\mathrm{p}}^{0}(p, T) / \mathrm{L} \cdot \mathrm{mol}^{-1} \cdot \mathrm{s}^{-1}=1.88 \cdot 10^{7} \cdot \exp \left(\frac{-34300+2.7 \cdot(p / \mathrm{bar})}{\mathrm{R} \cdot(T / \mathrm{K})}\right) \\
& k_{\mathrm{t}}^{0}(p, T) / \mathrm{L} \cdot \mathrm{mol}^{-1} \cdot \mathrm{s}^{-1}=8.11 \cdot 10^{8} \cdot \exp \left(\frac{-4600-1.58 \cdot(p / \mathrm{bar})}{\mathrm{R} \cdot(T / \mathrm{K})}\right)
\end{aligned}
$$

Dabei ist R die universelle Gaskonstante (8.3144 $\mathrm{J} \mathrm{mol}^{-1} \mathrm{~K}^{-1}$ ). 
Um die Umsatzabhängigkeit von $k_{\mathrm{p}}$ und vor allem von $k_{\mathrm{t}}$ zu berücksichtigen wird die sogenannte reduzierte Viskosität $\eta_{\mathrm{r}}$ eingeführt:

$$
\ln \left(\eta_{\mathrm{r}}\right)=12.41 \cdot g_{\mathrm{P}}+8.52 \cdot \sqrt{g_{\mathrm{P}}}
$$

Mit Gleichung D.6 ergeben sich folgende Gleichungen zur Berechnung von $k_{\mathrm{p}}$ und $k_{\mathrm{t}}$ unter Reaktionsbedingungen:

$$
\begin{aligned}
& k_{\mathrm{p}}\left(p, T, g_{\mathrm{p}}, \eta_{\mathrm{r}}\right)=\frac{k_{\mathrm{p}}^{0}(p, T)}{1+\eta_{\mathrm{r}} \cdot \frac{k_{\mathrm{p}}^{0}(p, T)}{1.13 \cdot 10^{10}}} \\
& k_{\mathrm{t}}\left(p, T, g_{\mathrm{p}}, \eta_{\mathrm{r}}\right)=k_{\mathrm{t}}^{0}(p, T) \cdot\left[\frac{0.832}{\eta_{\mathrm{r}}}+8.04 \cdot 10^{-6} \cdot\left(1-g_{\mathrm{p}}\right) \cdot k_{\mathrm{p}}\left(p, T, g_{\mathrm{p}}, \eta_{\mathrm{r}}\right)\right]
\end{aligned}
$$

\section{Literatur für Anhang C}

[1] G. Luft, R. Steiner, Chemiker Zeitung 95 (1971) 11

[2]J. Schweer, Dissertation, Göttingen (1988) 


\section{Danksagung}

Herrn Prof. Dr. M. Buback danke ich herzlich für eine sehr interessante Themenstellung und die stete Förderung und Unterstützung dieser Arbeit.

Herrn Dr. H.-P. Vögele danke ich für die Diskussionsbereitschaft, sowie für die Hilfe in Spektrometerfragen.

Den Mitarbeitern der Institutswerkstätten gilt mein Dank für ihre Hilfsbereitschaft und Unterstützung bei der Wartung des Minitechnikums.

Ein besonderer Dank gilt Stephan Jauer für die ausgesprochen gute Zusammenarbeit bei der Durchführung der Experimente im Minitechnikum. Des Weiteren haben die gemeinsamen Diskussionen maßgeblich zum Gelingen dieser Arbeit beigetragen. Auch seine handwerklichen Fähigkeiten waren in einigen Lebenslagen sehr hilfreich.

Heike Rohmann danke ich für die gute Stimmung im Schreibraum wie im Keller. Messungen im Minitechnikum ohne Heike waren nur halb so schön.

Für die Einweisung im Umgang mit dem Computerprogramm Predici, sowie für die Hilfe bei Softwareproblemen bedanke ich mich bei Matthias Müller.

Dr. M. Kling und PD Dr. S. Schmatz danke ich für die Durchführung der DFT-Rechnungen.

Den Korrekturlesern Moritz Gadermann und Stephan Jauer danke ich für deren Ausdauer diese Arbeit zu lesen.

Allen übrigen Mitgliedern der Arbeitsgruppe Buback möchte ich für die freundschaftliche Zusammenarbeit und stete Hilfsbereitschaft danken.

Den Mitarbeitern der Firma Akzo Nobel, Dr. B. Fischer, Dr. A. v. Swieten, R. Gerritsen, Dr. J. Meijer, Dr. F. Hoogesteger, S. Put und B. d. Vries, danke ich für ihre Diskussionsbereitschaft und für die Bereitstellung der Peroxide. 
Der BASF AG schulde ich Dank für Bereitstellung des Ethens.

Für die Ermöglichung des Chemie-Studiums durch das BAföG danke ich der Bundesrepublik Deutschland.

Der Doppelkopfrunde (Matthias, Moritz und Stephan) gilt mein Dank für die unterhaltsamen aber nicht unbedingt erfolgreichen Abende.

Meinen Eltern und meiner Familie danke ich für Unterstutzung während der Studienzeit.

Allen meinen Freunden danke ich für die gelegentliche Ablenkung vom Studium.

Zum Schluss möchte ich mich bei meiner Freundin Jessica dafür bedanken, dass sie immer für mich da war. 


\section{Lebenslauf}

Persönliche Daten:

21.12.1975

geboren in Heide

21.12.1976

Staatsangehörigkeit: deutsch

Schulausbildung:

$1982-1986$

Grundschule in Tellingstedt

$1986-1995$

Gymnasium in Heide/Holstein

Juni 1995

Erwerb der Allgemeinen Hochschulreife

\section{Hochschulstudium:}

Okt. 1995 - Mai 2001

Okt. 1997

Mai 2001

April 2000 - Mai 2001

seit Aug. 2001

seit Aug. 2001

Jan. 2000 - Sep. 2001

seit Okt. 2001
Diplomstudiengang Chemie an der Georg-August Universität in Göttingen

Vordiplomsprüfung

Diplomprüfung

Diplomarbeit am Institut für Physikalische Chemie der GeorgAugust Universität Göttingen unter Anleitung von Herrn Prof. Dr. M. Buback: „Zerfallskinetik von tert-Alkylperoxyestern und Initiierungseffektivität von tert-Butylperoxypentanoat in der Hochdruck-Polymerisation von Ethen“

Anfertigung der vorliegenden Dissertation am Institut für Physikalische Chemie der Georg-August Universität Göttingen unter Anleitung von Herrn Prof. Dr. M. Buback

Mitarbeiter im Sonderforschungsbereichs 357 der Deutschen Forschungsgemeinschaft: „, Molekulare Mechanismen unimolekularer Prozesse“

wissenschaftliche Hilfskraft am Institut für Physikalische Chemie der Georg-August Universität Göttingen

wissenschaftlicher Mitarbeiter am Institut für Physikalische Chemie der Georg-August Universität Göttingen 